\title{
KINEMATIC SYNTHESIS OF PLANAR, SHAPE-CHANGING RIGID BODY MECHANISMS FOR DESIGN PROFILES WITH SIGNIFICANT DIFFERENCES IN ARC LENGTH
}

\author{
Dissertation \\ Submitted to \\ The School of Engineering of the \\ UNIVERSITY OF DAYTON \\ In Partial Fulfillment of the Requirements for \\ The Degree of \\ Doctor of Philosophy in Mechanical Engineering
}

By

Shamsul Anuar Shamsudin

Dayton, Ohio

May, 2013

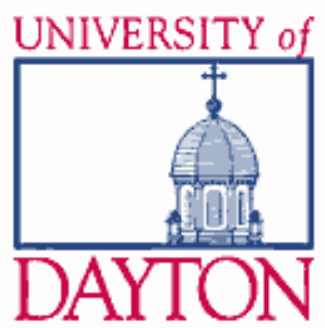


KINEMATIC SYNTHESIS OF PLANAR, SHAPE-CHANGING RIGID BODY MECHANISMS

FOR DESIGN PROFILES WITH SIGNIFICANT DIFFERENCES IN ARC LENGTH

Name: Shamsudin, Shamsul Anuar

APPROVED BY:

Andrew P. Murray, Ph.D.

Advisor Committee Chairman

Professor, Dept. of Mechanical and

Aerospace Engineering
Vinod K. Jain, Ph.D.

Committee Member

Professor, Dept. of Mechanical and

Aerospace Engineering
David H. Myszka, Ph.D., P.E.

Committee Member

Associate Professor, Dept. of

Mechanical and Aerospace Engineering
John G. Weber, Ph.D.

Associate Dean

School of Engineering
James P. Schmiedeler, Ph.D.

Committee Member

Associate Professor, Dept. of Aerospace and Mechanical Engineering, University of Notre Dame 


\section{(c) Copyright by}

\section{Shamsul Anuar Shamsudin}

All rights reserved

2013 


\begin{abstract}
KINEMATIC SYNTHESIS OF PLANAR, SHAPE-CHANGING RIGID BODY MECHANISMS FOR DESIGN PROFILES WITH SIGNIFICANT DIFFERENCES IN ARC LENGTH
\end{abstract}

Name: Shamsudin, Shamsul Anuar

University of Dayton

Advisor: Dr. Andrew P. Murray

Design of shape-changing machinery is an area of growing significance. Shape-change may be employed in the near future to vary the cross section of a wing, create flow-field control by altering shapes to locally affect downstream fluid behavior, or vary the size of a car seat to meet a wider array of ergonomic needs. Rigid body shape-change mechanisms offer many advantages including the high capacity to endure substantial loads while achieving large displacements. Their design techniques are also well-established. The goal of this research project is to develop the synthesis theory to address planar rigid-body shape-change where significant differences in arc length define the problem.

This dissertation presents a process to approximate several design profiles of significantly different arc lengths with rigid bodies connected by revolute and prismatic joints. This process is referred to as segmentation, and the initial step is the conversion of the design profiles into piecewise linear target profiles. Any two contiguous points on a target profile define a piece. Target profiles have the same approximate piece-length throughout. 
This is followed by segmentation which serves to identify the contiguous sets of pieces that are best approximated by either a rigid body $\mathscr{M}$-segment or a constant curvature $\mathscr{C}$-segment that contains a prismatic joint.

To facilitate segmentation, the concept of segment matrix is introduced. A segment matrix identifies the lengths of the bodies in the sequence of $\mathscr{M}$ - and $\mathscr{C}$-segments along a profile. The segmentation process is applied to open, closed, and fixed-end design profiles. A MATLAB-based tool was developed to facilitate visual assessment of the process and results. Finally, this dissertation illustrates five mechanization examples that apply the segmentation process, and the fundamental mechanism synthesis to guide the motion of the chain of rigid bodies to progress to the subsequent positions. 
To my parents, Halimah and Shamsudin,

my wife Asmida, and my children Adlina, Amri, and Syakir. 


\section{ACKNOWLEDGMENTS}

Graduate studies at the University of Dayton have been both fun and challenging. The classes have broadened my perspective as a mechanical engineering graduate, and provided me with the skills and knowledge needed to become an accomplished engineering instructor, advisor, and researcher. I also found the research group meetings where we shared updates, carried out small projects, and learned from each other, very beneficial. This interaction has contributed in many ways to the successful completion of this dissertation which represents the most important milestone on my road to graduation.

I would like to thank Professor Andrew Murray for his guidance, and also his patience during the extended period that I took to complete this journey. When obstacles came my way, he was always available with solutions to see me through. Many of my friends are of the opinion that I am fortunate to have him as my advisor, and I could not agree more.

I am also grateful to the other committee members for their invaluable contribution towards this research. Professor Vinod Jain guided me in his class and encouraged me to complete my studies. I thank Professor David Myszka for being supportive in the group meetings and for being instrumental in the writing of the technical papers we worked on. I also wish to thank Professor James Schmiedeler for his involvement in my doctoral research work and publications.

I would like to express my appreciation for the ever so generous financial support I received from the Ministry of Higher Learning of Malaysia and the Universiti Teknikal Malaysia Melaka(UTeM). 
I look forward to serving the country as an educator and sharing the knowledge of machine theory with the future generations of mechanical engineers.

I am grateful to fellow researchers at the Design of Innovative Machines Laboratory (DIMLab): Jonathan Lauden, Ali AlMandeel, 'Chef' Lin Li, Tong YuXuan, Jo Binjue Li, Dr. Dave A. Perkins, Andrew Hazlett, Brandon Leedy, Joshua Nieman, Sarotham Reddy and Linda Leben for keeping me company at the lab and sharing their ideas. I thank Kai Zhao of the University of Notre Dame for his help in the mechanization issues. I am also indebted to Dr. Aaron Altman and Dr. Markus P. Rumpfkeil for their insights on aircraft wings. I am grateful to Rise' Kreitzer, Capt. Edward Seaman, Constance Norman, Dr. Nicoletta Hary, and all the staff at the Roesch and Marian Library where I was engaged on a part-time basis.

My family has played a major role in this endeavor and I would like to thank my wife Asmida and my children Nurul Adlina, Khairul Amri, and Ahmad Syakir for their love, support, and understanding. I am forever indebted to them all. I am also aware that my parents, Halimah and Shamsudin, constantly include me in their prayers, and it is my hope that this achievement brings them much joy.

Lastly, but most importantly, I thank The Almighty God for His countless blessings, and for providing me with the determination to complete my studies and achieve my goal. With this achievement I pray for the ability to harness my expertise in this field for the education of future generations. 


\section{TABLE OF CONTENTS}

Page

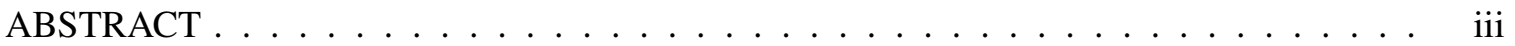

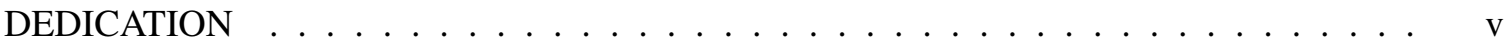

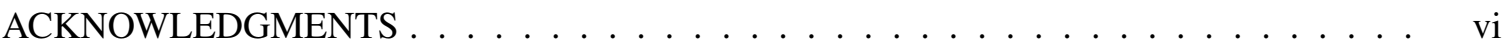

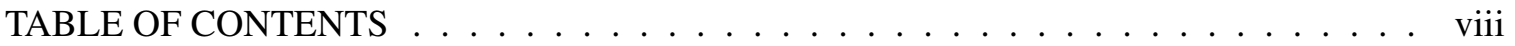

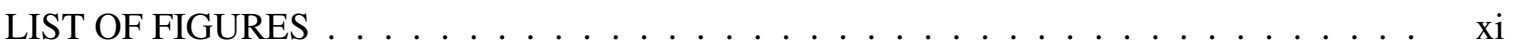

LIST OF TABLES $\ldots \ldots \ldots \ldots \ldots \ldots \ldots \ldots \ldots \ldots \ldots \ldots \ldots$ xviii

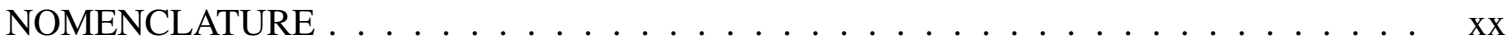

\section{CHAPTERS:}

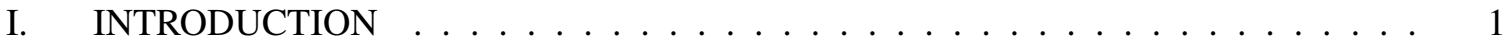

1.1 State of the Art in Shape-Change . . . . . . . . . . . . . . . . . . 1

1.1.1 Airfoils and Blades . . . . . . . . . . . . . . . . 1

1.1.2 Ship and Boat Hulls . . . . . . . . . . . . . . . 5

1.1.3 Antenna and Mirror . . . . . . . . . . . . . . . . . 5

1.1.4 Convertible Structures . . . . . . . . . . . . . . . . 6

1.1.5 Robotic Gripper and Prosthetics . . . . . . . . . . . . . . . 8

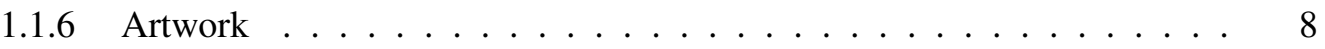

1.2 Various Shape-Changing Methods . . . . . . . . . . . . . . . . . . 9 9

1.2.1 Rigid-Body Shape-Change . . . . . . . . . . . . . . . 9

1.2.2 Compliant Mechanisms . . . . . . . . . . . . . . . . . 12

1.2.3 Membranes and Smart Materials . . . . . . . . . . . . . . . . 13

1.2.4 Combinations Involving Membranes and Rigid Bodies . . . . . . . . . . . . . 14

1.3 Rigid-Body, Shape-Changing, Mechanism Design Methodology . . . . . . . . . 14

1.3.1 Profiles and Segmentation . . . . . . . . . . . . . . . . . . . . 15

1.3.2 Mechanization Methods . . . . . . . . . . . . . . . 16

1.3.3 Limitations of This Method . . . . . . . . . . . . . . . . 18

viii 
1.4 Shape-Changing for Different Arc-Length Profiles . . . . . . . . . . . . . . . 19

1.4.1 Different Arc Length Examples . . . . . . . . . . . . . . . . . . . . . . . 19

1.4.2 New Profile Generation . . . . . . . . . . . . . . . . . 23

1.4.3 New Segmentation Method . . . . . . . . . . . . . . . . . 24

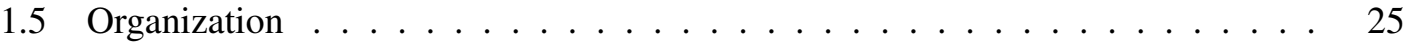

II. OPERATIONS ON TARGET PROFILES $\ldots \ldots \ldots \ldots \ldots \ldots \ldots$

2.1 Target Profile Generation . . . . . . . . . . . . . . . . . . . . 26

2.2 Metrics on Target Profiles . . . . . . . . . . . . . . . . 31

2.2.1 The Closed-Form Similarity Transformation Solution _ . . . . . . . 36

2.2 .2 Example . . . . . . . . . . . . . . . . . . 39

2.3 Curvature Calculations . . . . . . . . . . . . . . . . . . . 41

2.3.1 Calculating the Curvature of Target Profiles . . . . . . . . . . . . . 41

2.3.2 Curvature Smoothing . . . . . . . . . . . . . . . . . 42

2.3.3 Regenerating the Target Profile . . . . . . . . . . . . . 43

III. THE SEGMENTATION PROCESS . . . . . . . . . . . . . . . . . . . . . . . 48

3.1 A New Segmentation Process for Target Profiles of Different Arc Lengths . . . . 48

3.1 .1 The Segment Matrix . . . . . . . . . . . . . . . . . . . 50

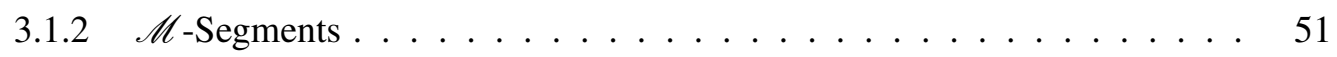

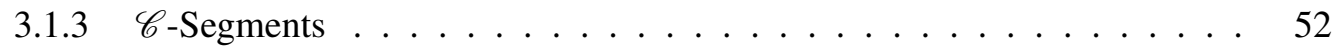

3.2 Evaluating and Adjusting the Segment Matrix . . . . . . . . . . . . . 54

3.3 Joining the Chain . . . . . . . . . . . . . . . . 58

3.4 Compound Segment Types . . . . . . . . . . . . . . . . . . 61

3.5 Other Profile Examples . . . . . . . . . . . . . . . . . . 63

3.5.1 Closed-Profile Example . . . . . . . . . . . . . . . . 63

3.5 .2 Fixed-End Profile Examples . . . . . . . . . . . . . . . . . 63

3.6 Comparing the Number of Joints . . . . . . . . . . . . . . . . . . . 65

3.7 Versatility of the Segmentation Method . . . . . . . . . . . . . . . 66

3.8 Automating the Segmentation Process . . . . . . . . . . . . . . . . . . 68

IV. SOFTWARE IMPLEMENTATION $\ldots \ldots \ldots \ldots \ldots \ldots \ldots \ldots$

4.1 Design Profiles . . . . . . . . . . . . . . . . . . . . 72

4.2 Target Profiles . . . . . . . . . . . . . . . . . . . 73

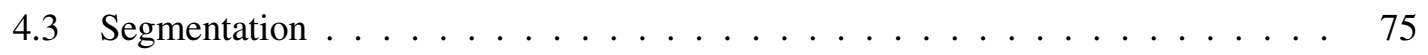

4.4 Visualization of Segmentation Results . . . . . . . . . . . . . . . . . 77

4.5 Compound Segments . . . . . . . . . . . . . . . . . . 77

V. SEGMENTATION AND MECHANIZATION EXAMPLES . . . . . . . . . . . . 79

5.1 Open Profile Examples . . . . . . . . . . . . . . . . . . . 79 
5.1 .1 Car Seat . . . . . . . . . . . . . . . . 80

5.1.2 U-to-D Transformation . . . . . . . . . . . . . . . 85

5.2 Fixed-End Example . . . . . . . . . . . . . . . . . . . . . . . . . . 89

5.3 Closed-Loop Example . . . . . . . . . . . . . . . . . . . . . 96

5.3 .1 Morphing Wing . . . . . . . . . . . . . . 96

5.3 .2 Flow-Field Control . . . . . . . . . . . . . . . . . . . . . 96

VI. CONCLUSIONS AND FUTURE WORK . . . . . . . . . . . . . . . . . 106

6.1 Contributions . . . . . . . . . . . . . . . . . . . 106

6.2 Future Work . . . . . . . . . . . . . . . . . . . . . . . . . 109

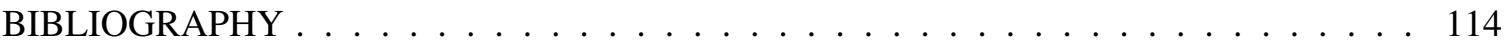
APPENDICES:

A. MATLAB IMPLEMENTATION OF THE SEGMENTATION PROCESS $\ldots \ldots \ldots$

A-1 Generating Design Profiles . . . . . . . . . . . . . . . . . . 123

A-1.1 Editing Design Profiles . . . . . . . . . . . . . . . 127

A-2 Generating Target Profiles . . . . . . . . . . . . . 136

A-3 Segmentation . . . . . . . . . . . . . . . . . . . 140 


\section{LIST OF FIGURES}

Figure $\quad$ Page

1 An early concept of a compliant mechanism for a morphing wing slat and flap designed for the F-111 fighter aircraft $[5] . \ldots \ldots \ldots 2$

2 A morphing wing provides only the amount of lift necessary, reducing the fuel use

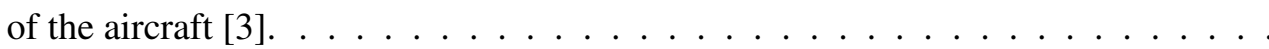

3 A shape-changing spoiler can be used to improve vehicle stability by providing additional downward force when navigating corners. . . . . . . . . . . . 3

4 The control surfaces in a submarine include (a) the starboard (tail) and (b) aft (front) hydroplanes of (c) the HMS Astute [28] . . . . . . . . . . . . . . . .

5 An underwater stabilizer uses two SMA actuators to move its flap. (a) The trailing edge is in a neutral position. (b) Joule heating causes the trailing edge to deflect upward. (c) Cooling of the top actuator returns the trailing edge to its neutral position

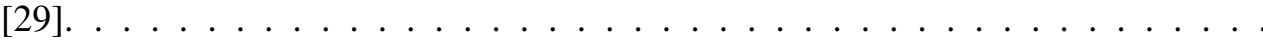

6 The shape-changing antenna can alter its shape during the scanning and focusing of a wave [33] . . . . . . . . . . . . . . . . . . . . .

7 A soft-top convertible roof $[40] \ldots \ldots \ldots \ldots \ldots$

8 A mobile stage and roof system in (a) stowed and (b), (c), and (d) are alternate deployed configurations $[14,41] \ldots \ldots \ldots \ldots \ldots$

9 A compliant mechanism holding a round object $[45] \ldots \ldots \ldots \ldots$

10 (a) Design profiles define the shapes of the letters. (b) A chain of rigid-bodies connected by $\mathrm{R}$ joints approximates the two letters. 
11 The mechanism is capable of moving the chain from approximating the " $U$ " to the " $D$ " as it progresses from (a) through (d) based on work in [46]. . . . . . . . . . . 10

12 The "skin" or outer layer of the shape-changing machine does not have to be rigid. The GINA has the skeleton mechanism underneath made of rigid members [73]. . . 15

13 A cable extends the length of the prosthetic finger and acts as an actuator [83]. . .

14 (a) A mean segment generated for points 1-2-3 of the two different length profiles. (b) Mapping the mean segments on the profiles does not show good results. . . . . 18

15 A conventional theoretical pressure distribution $C_{p}$ plot of a four-element wing. The leading-edge and trailing-edge flaps are in deployed positions. . . . . . . . . .

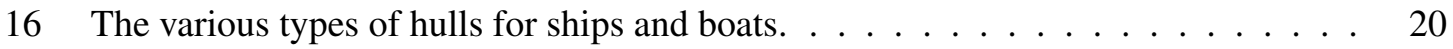

17 Different cross-sectional shapes along a modern ship's hull [26]. . . . . . . . . . 20

18 The Ti-Ni wires return to its original antenna shape by heating [66]. . . . . . . . . 21

19 (a) Schematic diagram of the multiple dies extrusion system. (b) The frame is axially twisted, and the width of the cross section varies steadily [87] . . . . . . . .

20 (a) Schematic diagram of the multiple dies pultrusion system. (b) The cross-sectional shapes vary gradually $[88] \ldots \ldots \ldots \ldots \ldots$

21 (a) Various cross-section shapes for the two extruded products. (b) The shapechanging die concept $[89] . \ldots \ldots \ldots \ldots \ldots$

22 Profile types include (a) open profiles, (b) closed profiles, and (c) fixed-end profiles.

23 Computation time decreases exponentially as $s_{d}$ increases in size. . . . . . .

24 Design profile (solid) with an approximating target profile (dashed) where points are positioned to give a constant arc length along the design profile. . . . . . . . .

25 Three design profiles with significantly different arc lengths represented with target profiles of nearly constant piece length. . . . . . . . . . . . . . 
26 (a) Three target profiles, with one deemed the reference profile. (b) Two profiles are transformed to the reference by a similarity transformation (with $b=1$ ). (c) The mean profile. (d) The mean profile transformed back to the original profile locations [46].

27 (a) Rigid bodies connected with revolute joints form a chain to closely approximate the profiles. (b) A mechanism design that moves the chain of rigid bodies between the three profiles. . . . . . . . . . . . . . . . .

28 (a) The reference points $\mathbf{Z}_{1_{i}}$. (b) The points $\mathbf{z}_{j_{i}}$ to undergo the similarity transformation. (c) The scaled, rotated and translated $\mathbf{z}_{2_{i}}$ that align with the $\mathbf{Z}_{1_{i}}$ for the optimal $b=0.72, \theta=-33.69^{\circ}$, and $\mathbf{d}_{j}=[-0.80,0.40]^{T}$. (d) The scaled, rotated and translated $\mathbf{z}_{2_{i}}$ that align with $\mathbf{z}_{1_{i}}$ for $b=1 \ldots \ldots \ldots$

29 Target profiles (a) with calculated curvature (b) and smoothed curvature (c). The dashed curves in (a) are regenerated profiles and are nearly identical to the original profiles. . . . . . . . . . . . . . . . . .

30 The location of $\tilde{\mathbf{z}}_{j_{i+1}}$ is reconstructed from $\tilde{\mathbf{z}}_{j_{i-1}}, \tilde{\mathbf{z}}_{j_{i}},\left(\tilde{\alpha}_{j_{i}}, \tilde{\beta}_{j_{i}}\right)$ and $\tilde{r}_{j_{i}} \ldots \ldots$

31 (a) The first segment from three target profiles to be represented by one mean segment. (b) Segments 2 and 3 shifted to reference segment 1 in a distance minimizing transformation. (c) The mean segment is generated as the average of the corresponding segment points. (d) The mean segment is shifted back to the original segments in another distance minimizing transformation. . . . . . . . . . . . . . .

32 A $\mathscr{C}$-segment is built and then transformed to its location in a distance minimizing

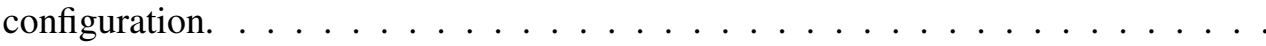

33 (a) The second segment from three target profiles approximated with a $\mathscr{C}$-segment. (b) An arc of the same radius, but varying length, approximates the segment on all

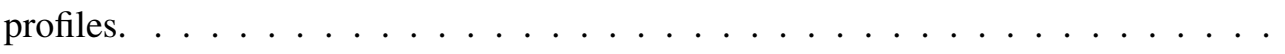

34 A rigid-body chain that approximates the target profiles. The chain after the initial segmentation matrix is shown in (a) with $E_{\max }=1.50$. The chain after the error-reducing iterations is shown (b) with $E_{\max }=0.38$. The inset illustrates that segmentation points are not coincident. . . . . . . . . .

35 After instances of segments are created, the segmentation points will not coincide. To merge the segmentation points and form a chain connected by revolute joints, each segment must be repositioned by adjusting $\tilde{\mathbf{z}}_{j_{1}}^{1}$ and $\tilde{\theta}_{e} \ldots \ldots \ldots \ldots$ 
36 The joining process unites segmentation points, yet changes the error from $E_{\max }=$

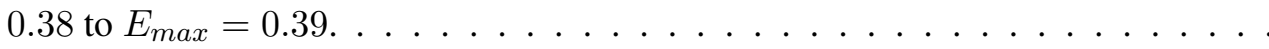

37 (a) The original chain consisted of 4 segments resulting in $E_{\max }=0.39$, yet one revolute joint exhibited limited motion. (b) The $2^{\text {nd }}$ and $3^{r d}$ segments were fused into a compound segment, resulting in a three-segment chain with $E_{\max }=0.45$. .

38 (a) The E420 and E850 profiles of airfoils. (b) The chain of ten segments with $E_{\max }=0.015$. (c) Fusing segments raises the error to $E_{\max }=0.017$ while reducing the number of joints. $\ldots \ldots \ldots \ldots \ldots$

39 Fixed-end profiles shown in (a). The final, rigid-body, shape-approximating chain is shown in (b) having $E_{\max }=0.18 \ldots \ldots \ldots \ldots \ldots$

40 (a) The $[\mathscr{M} \mathscr{C} \mathscr{M} \mathscr{M} \mathscr{M}]$ chain is selected with an after-assembly error of $E_{\max }=$ 0.26 . (b) A compound segment increases maximum error to $E_{\max }=0.28$, which is still lower than the $[\mathscr{M} \mathscr{C} \mathscr{M} \mathscr{M}]$ design with $E_{\max }=0.37$ in (c). . . . . . .

41 (a) The design profiles. (b) The $[\mathscr{C} \mathscr{C} \mathscr{M} \mathscr{C} \mathscr{C} \mathscr{M} \mathscr{M} \mathscr{C} \mathscr{C} \mathscr{C} \mathscr{M} \mathscr{C} \mathscr{C} \mathscr{M} \mathscr{C}]$ segmentation with $E_{\max }=0.24 \ldots \ldots \ldots \ldots \ldots \ldots \ldots$

42 The range from 0 to 0.2 is an acceptable range to be approximated by an arc of

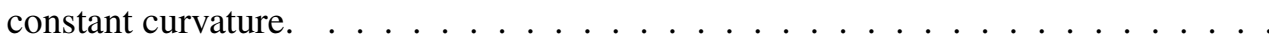

43 In order to identify a potential region in which to insert another prismatic joint, the curvature distribution (from Fig. 42) is replotted starting with the end of the previously selected curvature band. . . . . . . . . . . . . .

44 (a) A design profile and its corresponding target profile created with 10 points. (b) The plot of the target profile's arc length as a function of its number of points. . .

45 (a) The silhouettes of a small, an average and a large driver [112]. (b) Seat design profiles that ideally suit the small, average and large drivers from (a). . . . . . . . .

46 The three seat profiles can be approximated by the five segment shape-approximating chain in (a). The creation of a compound segment reduces the chain to three segments in (b) with only a visually-acceptable increase in error. . . . . . . . . . . .

47 (a) The seat accommodates the $1^{\text {st }}$ percentile group, (b) the $50^{\text {th }}$ percentile group, and (c) the $99^{t h}$ percentile group. . . . . . . . . . . . . .

48 Labels for the dyads and revolute joints for the car seat example. . . . . . . . . . 
49 (a) The same arc length design profiles for $\mathrm{U}$ and $\mathrm{D}$. (b) The new profiles include an enlarged "D" so that its overall size is comparable to that of "U." . . . . . . . . . . 86

50 Segmentation shows good approximations with a maximum point-to-point error

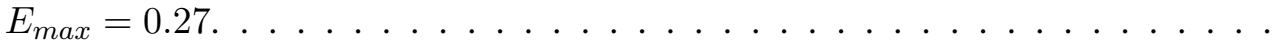

51 (a) The mechanism aligned with the "U." (b) An interim position close to the "U." (c) An interim position close to the "D." (d) The mechanism aligned with the "D." .

52 Labels for the dyads and revolute joints for the UD example. . . . . . . . . .

53 (a) The NASA 30P30N wing is shown with its slat in the stowed configuration. (b) The slat in the deployed configuration. (c) The slat locations have been used to identify a fixed-end design challenge.

54 The two rack and pinion systems drive the slat out of the main wing [114]. . . . . .

55 (a) Fluctuating instantaneous sound pressure field [115]. (b) Many early types of slats are hinged to the main wing element $[116] \ldots \ldots \ldots \ldots$. . . . . . .

56 (a) The target profiles generated with $s_{d}=0.1$. (b) The four-segment design has an $E_{\text {max }}=0.14$. (c) The error minimizing segmentation result with a $[\mathscr{C} \mathscr{M} \mathscr{C}]$ chain

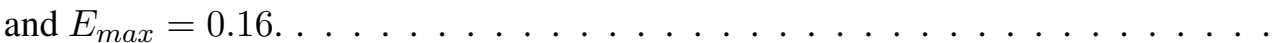

57 (a) A fully stowed slat. (b) The slat starts to deploy. (c) An interim position as it approaches the deployed position. (d) The fully deployed (extended) slat. . . . . . .

58 Labels for the dyads and revolute joints for the wing slat example. . . . . . . . .

59 (a) Thin E850 high-speed airfoil and thick E420 high-lift airfoil. (b)-(f) Progression of the morphing wing between E420 and E850 airfoils. The two straight blue lines at the tail are paths for two sliders that guide the $\mathscr{C}$-segment. The curved sliders in red follow a curved path as it guides the other end of the $\mathscr{C}$-segment. . . . . . . . . 98

60 Labels for the dyads and revolute joints for the morphing airfoil example. . . . . . 98

61 (a) The three profiles of the original flow field example. (b) The new profiles have significant differences in arc lengths. . . . . . . . . . . . . . . . . . . . 100

62 The chains of six segments are shown here in the two designs, with (a) imposing symmetry between segments in top half of the chain and those in the bottom half. . 102 
63 (a) The mechanism aligned with the ellipse. (b) An interim position between the ellipse and the tear-drop. (c) The mechanism aligned with the tear-drop. (d) An interim position between the tear-drop and the circle. (e) The mechanism aligned with the circle. . . . . . . . . . . . . . . . . . .

64 (a) A slider and a slot that can be entrenched in the floor or frame. (b) A telescoping element that can operate from inside the device for flow-field control [118]. . . . . 104

65 Labels for the dyads and revolute joints for the flow-field example. . . . . . . . . .

66 (a) The mechanization challenges posed by (a) and (b) are significantly different. Associating the identified point as being at the same location in the segmentation in (c) may be an accurate way of approximating bodies, but poses a significant mechanization challenge. . . . . . . . . . . . . .

67 Initial results for a compliant mechanism with living hinges, approximating three profiles. . . . . . . . . . . . . . . . . . . . . 113

A-1 The first two windows in ShapeChanger. . . . . . . . . . . . . .

A-2 (a) The first design profile is sketched. (b) Identifying that another profile is to be added. (c) The second design profile is sketched. (d) Proceed to the editing of the

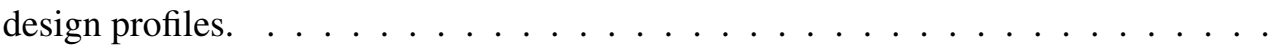

A-3 (a) A closed design profile is generated in the workspace with left mouse clicks. (b) A right click closes and finishes the profile. . . . . . . . . . .

A-4 (a) A fixed-end design profile is generated in the workspace with left mouse clicks. The second control point of the next profile is clicked. (b) The second profile adopts the same initial point as the first profile, and the connection to it is automatic. (c) The second profile adopts the same final point as the first profile, and the connection to it is automatic with the right click indicating completion of the profile. . . . . . . 128

A-5 Editing operations on the design profiles. . . . . . . . . .

A-6 (a) A click selects and highlights that profile. A second click indicates that point is to be moved. (b) The curve after a new location is selected for the point. . . . . . .

A-7 (a) A click selects and highlights that profile. (b) A second click selects and deletes the control point. . . . . . . . . . . . . . . . . 131 
A-8 (a) A click selects and highlights the profile. A second click indicates the range marked by two magenta squares. (b) If the range is right, select the exact location of the new point. Then the new point appears on the profile after the selection. . . .

A-9 (a) Select the curve and then the reference point (b) Click on the new location for the selected reference point on the profile to move the entire profile. . . . . . . . . 133

A-10 (a) The top profile is selected, and a second click indicates the control point about which to rotate. A dialog box opens to input the angle of rotation. (b) The curve after a rotation about the selected control point. . . . . . . . . . . . . . . 135

A-11 (a) A pair of profiles is (b) rescaled to have the same arc length. . . . . . . . . 135

A-12 The workspace presents the two target profiles matching well the design profiles beneath them. . . . . . . . . . . . . . . . . . . 136

A-13 The relevant information about target profiles influence the next phase, the segmen-

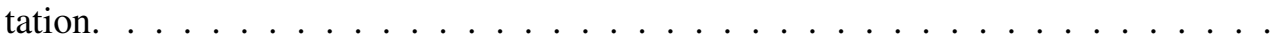

A-14 A selection of desired piece length, $s_{d}=4$, that leads to a poor match between the design and target profiles. . . . . . . . . . . . . . . . . . 138

A-15 Segmentation process takes the input from this window. . . . . . . . . . . 141

A-16 (a) Reset starting points dialog box. (b) Original starting points of the design profiles. (c) New starting points for the target profiles. . . . . . . . . . . . . . . . . . 142

A-17 (a) A user can set whether to see the optimization process changing the segments' lengths, or (b) to see the result of each chain design, or just see the final result. . . .

A-18 A result from the segment synthesis that is selected due to it having the lowest maximum point-to-point error, $E_{\max }=0.09 \ldots \ldots \ldots \ldots$

A-19 (a) Maximum angle variations at revolute joints are shown and an allowable angle is set. (b) Fusing segments can reduce complexities. . . . . . . . . . . . . . . . 145 


\section{LIST OF TABLES}

1 Design vector errors for all five-jointed chains. . . . . . . . . . . . . 66

2 Target profile data for the "UDAYTON" example shown in Fig. $41 \ldots \ldots 8$

3 Control points used in constructing the car seat profiles. . . . . . . . . . . 81

4 The mechanism's revolute joints for the car seat example in the configuration shown in Fig. 48. . . . . . . . . . . . . . . . . . . . . . . . . 84

5 The revolute joints in the segmented chain for the car seat example in the configuration shown in Fig. 48 . . . . . . . . . . . . . . . . . . . . . . . 84

6 Control points used in constructing the $\mathrm{U}$ and $\mathrm{D}$ profiles. . . . . . . . . . 85

7 The mechanism's revolute joints for the UD example in the configuration shown in

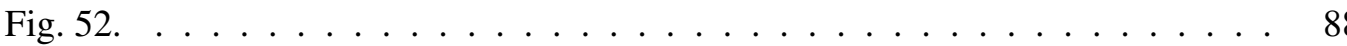

8 The revolute joints in the segmented chain for the UD example in the configuration shown in Fig. 52. . . . . . . . . . . . . . . . . . . . .

9 Control points that define the stowed and deployed profiles of the wing slat. . . . .

10 The revolute joints in the segmented chain for the wing slat example in the configuration shown in Fig. 58. . . . . . . . . . . . . . . . . . . 95

11 Points used in constructing the E850 and E420 profiles. . . . . . . . . . . . 97 
12 The mechanism's revolute joints for the morphing wing example in the configuration shown in Fig. 60 . . . . . . . . . . . . . . . . . . . 97

13 Mechanism points for the morphing airfoil example in the configuration shown in

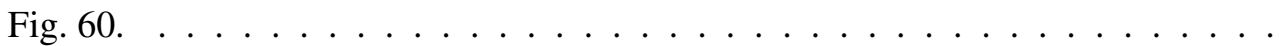

14 Revolute joints for the morphing airfoil example in the configuration shown in Fig. 60. 99

15 Points used in constructing the circle, ellipse, and tear drop profiles. . . . . . . . 101

16 The mechanism's revolute joints for the flow-field example in the configuration shown in Fig. 65. . . . . . . . . . . . . . . . . . . . 105

17 Segment and linkage joints for the flow-field example in the configuration shown in Fig. 65. . . . . . . . . . . . . . . . . . . . 105 


\section{NOMENCLATURE}

\begin{tabular}{|c|c|}
\hline $\mathscr{M}$ & Mean segment type \\
\hline $\mathscr{C}$ & Constant curvature segment type \\
\hline$j_{i}$ & Length of piece $i$ on design profile $j$ \\
\hline$J$ & Arc length of design profile $j$ \\
\hline$s_{j_{i}}$ & Length of piece $i$ on target profile $j$ \\
\hline$s_{d}$ & Desired piece length for target profiles \\
\hline$N$ & Number of points for a segment \\
\hline$n$ & Number of points for a profile in general \\
\hline$m_{j}$ & Number of pieces on target profile $j$ \\
\hline$n_{j}$ & Number of points on target profile $j$ \\
\hline$m_{j}^{e}$ & Number of pieces for $e^{t h}$ segment on profile $j$ \\
\hline$n_{j}^{e}$ & Number of points for $e^{t h}$ segment on profile $j$ \\
\hline$\epsilon_{s}$ & $\begin{array}{l}\text { Error representing the difference between the average segment length } \\
\text { and the desired piece length }\end{array}$ \\
\hline$m_{j}^{*}$ & Number of pieces on target profile $j$ that minimizes $\epsilon_{s_{j}}$ \\
\hline$n_{j}^{*}$ & Number of points on target profile $j$ that minimizes $\epsilon_{s_{j}}$ \\
\hline$S_{j}$ & Arc length of target profile $j$ \\
\hline & Average length of all $m_{j}^{*}$ linear pieces on all $p$ target profiles \\
\hline
\end{tabular}




\begin{tabular}{|c|c|}
\hline $\mathbf{Z}_{1_{i}}$ & A set of $i$ points on a reference profile \\
\hline $\mathbf{z}_{j_{i}}$ & A set of $i$ points on profile $j$ that will be transformed \\
\hline $\mathbf{Z}_{1_{t}}$ & A vector composed of $Z_{1 x t}=\sum Z_{1 x i}$ and $Z_{1 y t}=\sum Z_{1 y i}$ for $i=1, \ldots, N$ \\
\hline $\mathbf{z}_{j_{t}}$ & A vector composed of $z_{j x t}=\sum z_{j x i}$ and $z_{j y t}=\sum z_{j y i}$ for $i=1, \ldots, N$ \\
\hline$b$ & Scale factor in the similarity transformation \\
\hline$\theta$ & Angle of rotation in the similarity transformation \\
\hline $\mathbf{A}_{j}$ & Rotation matrix in the similarity transformation \\
\hline $\mathbf{d}_{j}$ & Translation vector in the similarity transformation \\
\hline$D$ & The average squared point deviation between a profile or \\
\hline & a segment with its reference \\
\hline$\kappa_{j_{i}}$ & Curvature of point $i$ on target profile $j$ \\
\hline$\left\{\alpha_{j_{i}}, \beta_{j_{i}}\right\}^{T}$ & The center of a circle passing through $\mathbf{z}_{j_{i-1}}$ \\
\hline & $\mathbf{z}_{j_{i}}$ and $\mathbf{z}_{j_{i+1}}$ \\
\hline$r_{j_{i}}$ & Radius of curvature of point $i$ on target profile $j$ \\
\hline$\phi$ & Angle $\angle \mathbf{z}_{j_{i-1}} \mathbf{z}_{j_{i}} \mathbf{z}_{j_{i+1}}$ \\
\hline MAV & A micro unmanned aerial vehicle \\
\hline SMA & Shape-memory alloy \\
\hline SME & Shape-memory effect \\
\hline DOF & Degree(s) of freedom for a mechanism \\
\hline $\mathbf{V}$ & A design vector that shows the sequence of $\mathscr{M}$ - and $\mathscr{C}$-segments \\
\hline$S M$ & A segment matrix \\
\hline$E M$ & A segment error matrix \\
\hline$A M$ & A joint angle matrix \\
\hline$X^{T}$ & Transpose of a matrix $X$ \\
\hline
\end{tabular}




$\begin{array}{ll}\mathbf{P}_{j_{i-1}} & \text { A direction vector that extends from } \mathbf{z}_{j_{i-1}} \text { to } \mathbf{z}_{j_{i}} \\ \tilde{\mathbf{z}}_{j_{i}} & \text { A regenerated profile point based on radius of smoothed curvature } \\ & \text { and uniform pieces } \\ \tilde{r}_{j_{i}} & \text { Radius of smoothed curvature of point } i \text { on target profile } j \\ \tilde{\kappa}_{j_{i}} & \text { Smoothed curvature of point } i \text { on target profile } j \\ \left\{\tilde{\alpha_{j}}, \tilde{\beta}_{j_{i}}\right\}^{T} & \text { The circle center for finding } \tilde{\mathbf{z}}_{j_{i+1}} \\ p & \text { Number of design profiles } \\ q & \text { Number of segments in a chain of rigid bodies } \\ \alpha & \text { Minimum number of pieces per segment } \\ \bar{r}^{e} & \text { Mean radius for the } e^{t h} \mathscr{C} \text {-segments } \\ \delta & \text { Step angle in constructing a } \mathscr{C} \text {-segment } \\ E_{j}^{e} & \text { Error calculated for } e^{t h} \text { segment on profile } j \\ \bar{E}^{e} & \text { Mean error calculated for } e^{t h} \mathscr{M} \text {-segment on all profiles } \\ \delta m_{j}^{e} & \text { Change in number of pieces for } e^{t h} \text { segment on profile } j\end{array}$




\section{CHAPTER I}

\section{INTRODUCTION}

\subsection{State of the Art in Shape-Change}

Machines can benefit from the capacity to vary between specific shapes in a controlled manner. Although there are numerous examples of conventional machines that exhibit shape-change, novel approaches are currently being developed to solve increasingly sophisticated problems and address a variety of new applications. This chapter presents an overview of this research work and places the new research generated in this dissertation in its context.

\subsubsection{Airfoils and Blades}

The focus of much study has been on shape morphing aircraft wings that increase performance over a range of flight conditions [1,2]. Most of the design work has centered on changes between wing profiles of similar arc length $[3,4,5]$. The fundamentals of aerodynamics dictate, however, that lift and drag can be significantly altered with a change in camber and chord [6]. That is, for high lift situations (e.g., approach, landing, and climb), a higher camber and longer absolute chord are desirable, whereas for efficient cruising, a lower camber and shorter chord are desirable. Kota et al. [5] discovered an early shape-changing mechanism design using rigid-links and fiberglass flex-panels for a leading-edge slat and a trailing-edge flap of a fighter aircraft wing that achieved 
superior aerodynamic benefits, as shown in Fig. 1. However, in that work, no significant change in arc length of the airfoil was considered.

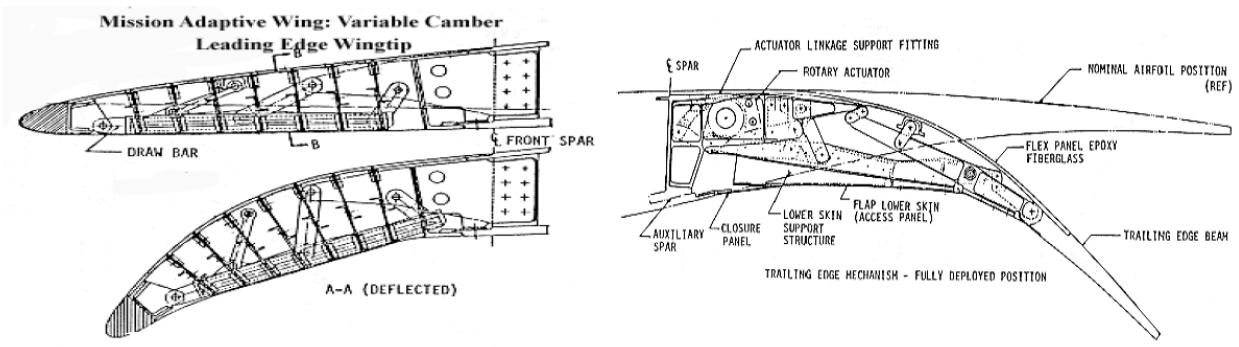

Figure 1: An early concept of a compliant mechanism for a morphing wing slat and flap designed for the F-111 fighter aircraft [5].

Abdulrahim et al. [7] studied a membrane wing for a micro unmanned aerial vehicle (MAV). The under cambered membrane wing has a wingspan of 24 inches. Such a wing changes its shape by morphing its entire profile by wire control rods connected to small motors in the fuselage. Limaye et al. [8] incorporated compliant kits of beams and connectors to model the trailing edge of a morphing aircraft wing. The compliant beams are deformed to alter the shape of the wing. Strelec and Lagoudas [9] used smart materials to control the shape of a reconfigurable wing to match a set of desired airfoil profiles. Weisshaar et al. $[10,11]$ underscored the importance of searching for new technologies to gain improved control surfaces for military aircrafts. They used smart materials to actuate the shape change in the trailing-edge flap of an aircraft wing. Kota et al. [5, 12] and Santer et al. [13] used compliant mechanisms to change the shape of a control surface that approximates an airfoil. Kota et al. [12] reported an increase in lift while maintaining a nearly constant drag coefficient. Another shape-changing wing design is illustrated in Fig. 2 in which the wing morphs shape as the aircraft burns fuel and less lift is required [3]. In addition to aircraft, airfoils may be used as automotive spoilers [14]. Figure 3 shows a shape-changing automotive spoiler composed 
of rigid-body segments. The spoiler can actively change shape as the need for traction varies. On straight stretches of highways, lower traction caused by a low pitch angle in the spoiler results in better fuel efficiency, whereas in curves, a high pitch angle in the spoiler produces higher downward force for better grip on the road [15].

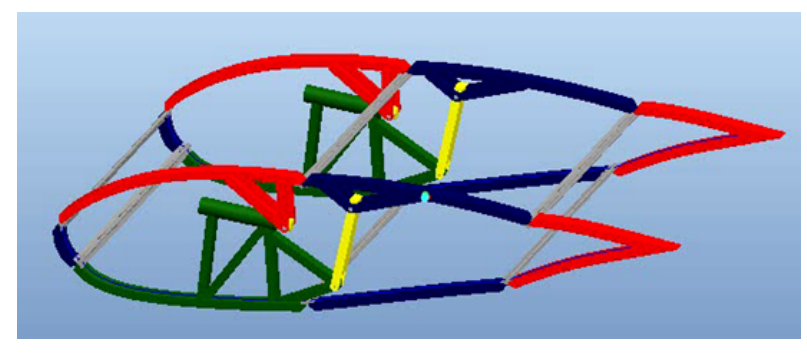

Figure 2: A morphing wing provides only the amount of lift necessary, reducing the fuel use of the aircraft [3].

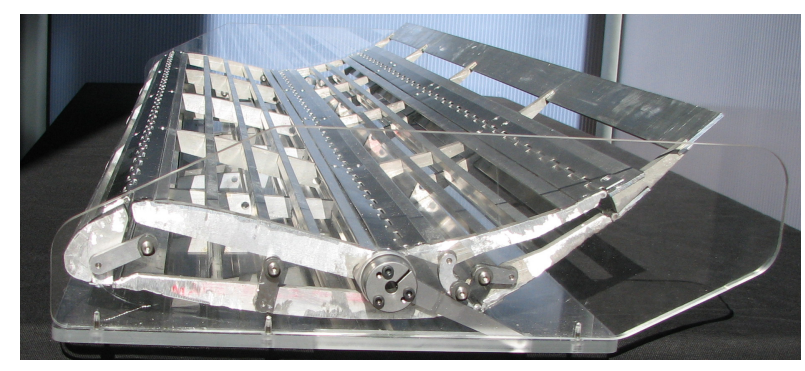

Figure 3: A shape-changing spoiler can be used to improve vehicle stability by providing additional downward force when navigating corners.

Cesnik et al. [16], Wong [17], and Grohmann et al. [18] investigated the use of smart materials like active fiber composites and piezoceramics to morph the shapes of helicopter blades. The US military, Sikorsky Helicopters, and DARPA are among organizations interested in developing shapemorphing rotor blades [19]. Similarly, wind turbine blades, also based on airfoils, were studied by Barlas et al. [20], Gaunaa [21], and Lambie [22]. They integrated moving flaps at the leading 
and trailing edges of the airfoil to change blade shapes according to the speed of the wind. The anticipated optimized performance achieved by integrating active flow control in the wind turbine rotors is presented in $[23,24]$.

Hydroplanes (sometimes called "underwater wings") and rudders can benefit from shape-changing [25]. In surface ships, active fin stabilizers or gyrofins, play a major role in preventing capsizing due to pitching and rolling moments [26]. Molland [27] explained that as a submarine operates underwater at a speed above ten knots, its stability and control depend predominantly on the hydrodynamic forces and moments on the hull and control surfaces. To remedy these problems, two sets of hydroplanes are needed. Figure 4 illustrates their locations. Gillmer mentions that some un-

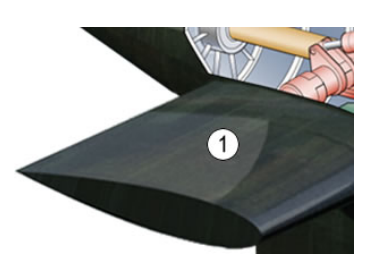

(a)

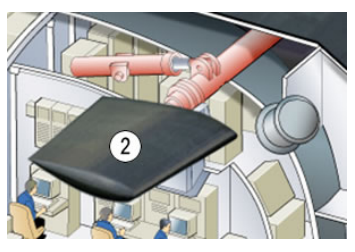

(b)

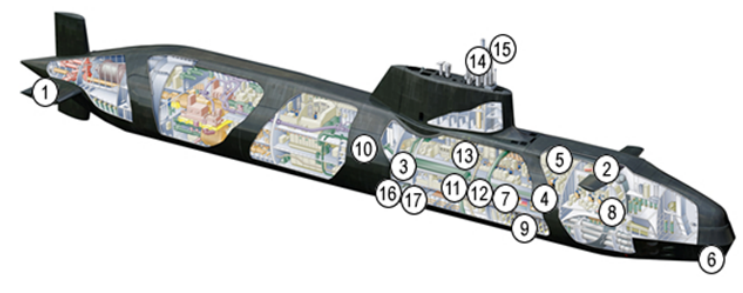

(c)

Figure 4: The control surfaces in a submarine include (a) the starboard (tail) and (b) aft (front) hydroplanes of (c) the HMS Astute [28].

derwater stabilizers can have trailing-edge flaps that produce the most effective vertical force [26].

To control such flaps on underwater stabilizers, Garner et al. [29] presented the development of a biomimetic active hydrofoil that utilized shape-memory alloy (SMA) actuator technology. They investigated the use of $0.58 \mathrm{~mm}$ diameter wires made of nickel-titanium with $10 \%$ copper on the outside of the $2 \mathrm{~m}$ long gyrofin profile, as shown in Fig. 5. 


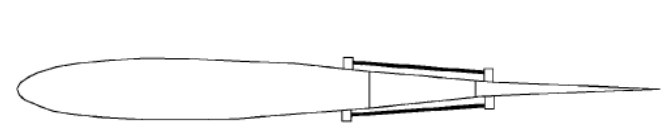

(a)

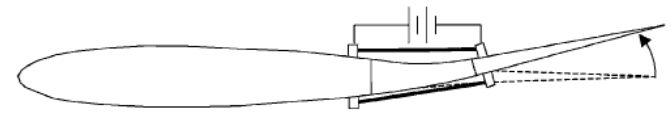

(b)

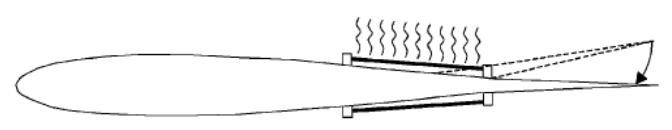

(c)

Figure 5: An underwater stabilizer uses two SMA actuators to move its flap. (a) The trailing edge is in a neutral position. (b) Joule heating causes the trailing edge to deflect upward. (c) Cooling of the top actuator returns the trailing edge to its neutral position [29].

\subsubsection{Ship and Boat Hulls}

Gernez et al. [30] developed Protei, which is an autonomous, shape-shifting, sailing vessel for ocean exploration and conservation. The flexible and segmented vessel resembles an airfoil when viewed from the top. The large rudder underneath is merged with the hull and changes shape to curl right or left as the boat captures the forces from the wind and sea currents. In these marine applications, different arc length shape-change may be practical to achieve the desired shapes.

\subsubsection{Antenna and Mirror}

Advances in electro-optics resulting from shape change include active aperture antennas [31] and deformable mirrors [32]. A compliant shape-changing antenna designed by Lu et al. [33, 34] is shown in Fig. 6. Furthermore, Plecnik [35] proposed a chain of rigid-bodies with reflective surfaces that can be adjusted to increase or decrease light intensity. Gupta [36] studied the application of piezoelectric actuators to steer a satellite antenna. The study used a Genetic Algorithm to optimally place PZT-5A actuators and the Finite Element Method to study the expected deformations. 


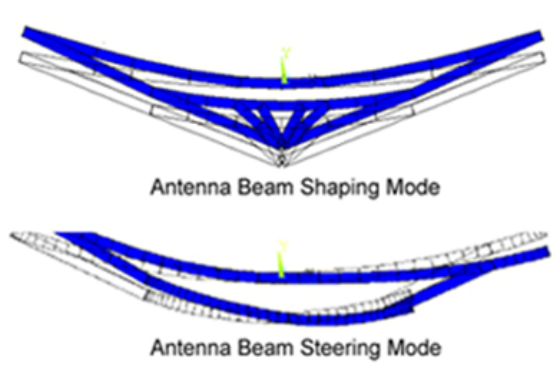

Figure 6: The shape-changing antenna can alter its shape during the scanning and focusing of a wave [33].

Satellites rely on the shapes of mirrors to capture clear images of the universe. Maji et al. [37] highlighted methods by which mirror pieces can be situated in the right position and orientation using smart material alloys that can be actuated to change shape via electrical signals. Stamper et al. [38] used membrane mirrors like electroformed nickel that are tensioned at discrete attachment points. Fernández and Artal [39] used a membrane deformable mirror with 37 electrodes to study its use as an adaptive optics element.

\subsubsection{Convertible Structures}

Automotive convertible roofs [40] and portable performance stages [14] involve shape changes in structural applications. Figure 7 shows a side view of a fully deployed convertible roof of a car. A shape-change problem may be defined by the deployed and stowed configurations of such a roof.

Erdman et al. [14] included the analysis problem of a portable performance stage that is shown in Fig. 8. The stage was designed to be large enough for live performances with an integrated canopy. The roof, composed of rigid-bodies, can achieve several configurations. 


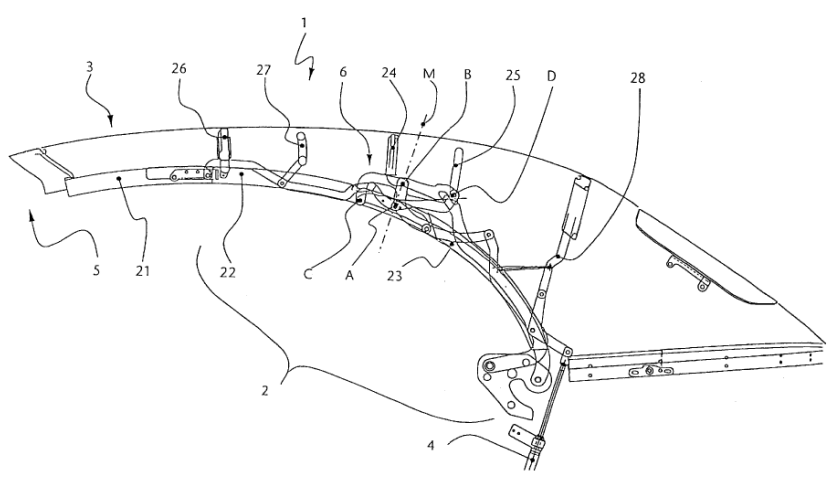

Figure 7: A soft-top convertible roof [40].

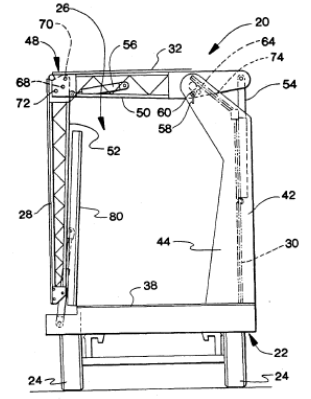

(a)

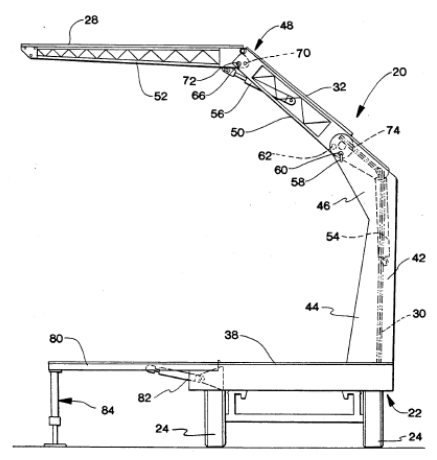

(c)

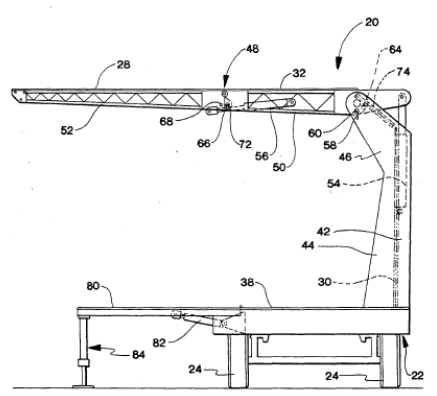

(b)

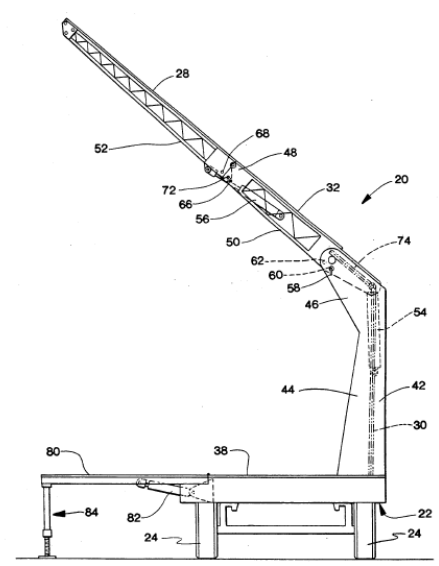

(d)

Figure 8: A mobile stage and roof system in (a) stowed and (b), (c), and (d) are alternate deployed configurations $[14,41]$. 


\subsubsection{Robotic Gripper and Prosthetics}

In manufacturing, robotic grippers are designed to move between prescribed shapes. Mohd Zubir et al. [42] used a compliant mechanism for a microgripper. Hirose and Umetani [43] developed an active shape-conforming gripper that could wrap around objects with round and concave shapes. A variable arc length gripper would be desirable to grasp objects of different sizes. Moon [44] used compliant mechanisms to approximate the shape of a human finger during a gripping motion. Petkovic et al. [45] used carbon-black filled silicon material that is able to morph the gripper to accommodate different objects, as shown in Fig. 9.
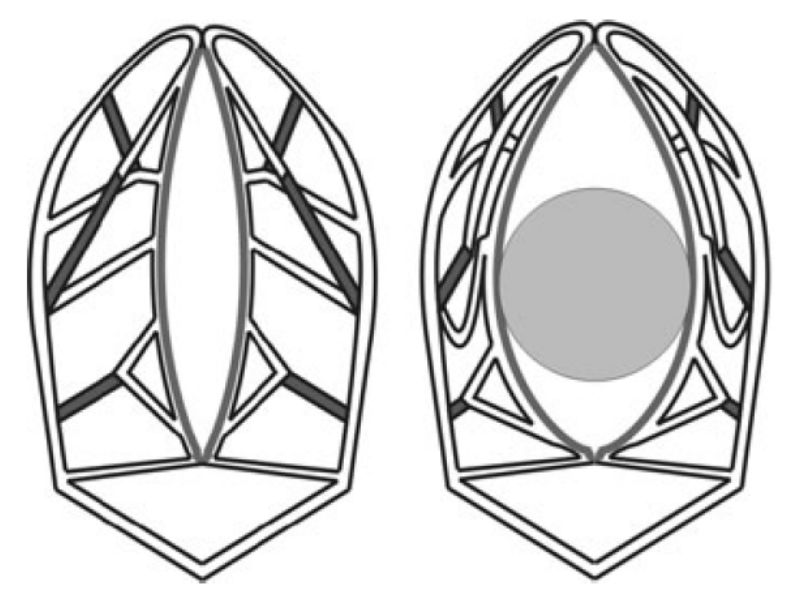

Figure 9: A compliant mechanism holding a round object [45].

\subsubsection{Artwork}

For entertainment purposes, a mechanism that morphs between the letters " $U$ " and " $D$ " has been proposed [46]. The profiles and the set of rigid bodies that morph between the two letters are shown in Figs. 10a and 10b, respectively. A single input, shown in Fig. 11a, actuates the mechanized chain. 


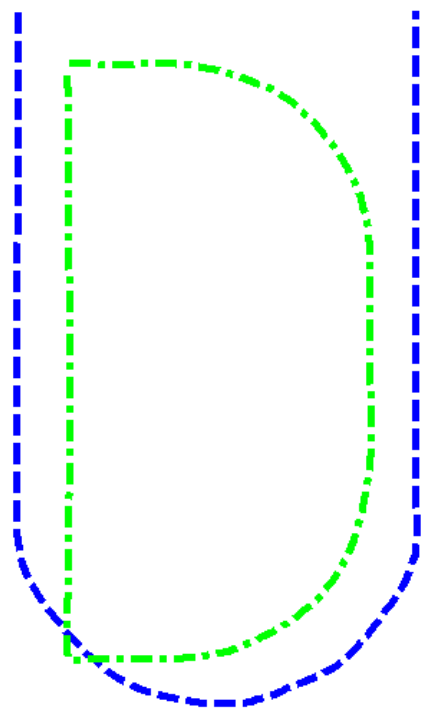

(a) Profiles

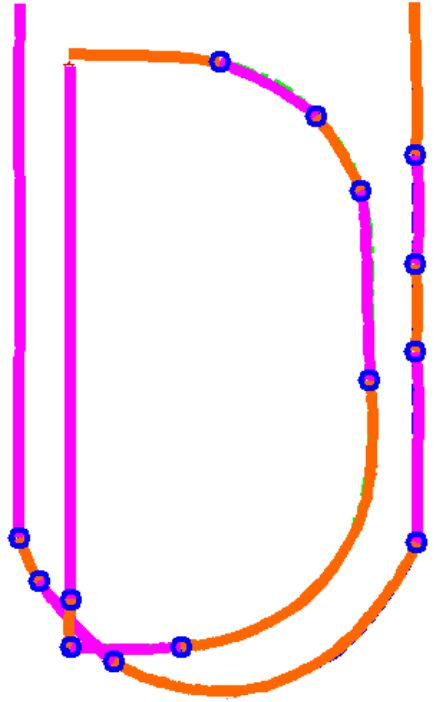

(b) Segments

Figure 10: (a) Design profiles define the shapes of the letters. (b) A chain of rigid-bodies connected by $\mathrm{R}$ joints approximates the two letters.

\subsection{Various Shape-Changing Methods}

There have been many emerging technologies developed for various applications. The main ones include rigid-body mechanisms, compliant mechanisms, shape-memory effect materials and membranes, as well as combinations of these with rigid-bodies.

\subsubsection{Rigid-Body Shape-Change}

Rigid body mechanisms are typically composed of familiar machine elements like linkages, gears, cams, axles, etc. The theories governing their analysis are well established, explaining accurately their kinematics and the forces encountered [47]. The design of systems composed of these elements also has many well established methodologies, with many of the components standardized and readily available from component manufacturers. In addition to the established analysis 


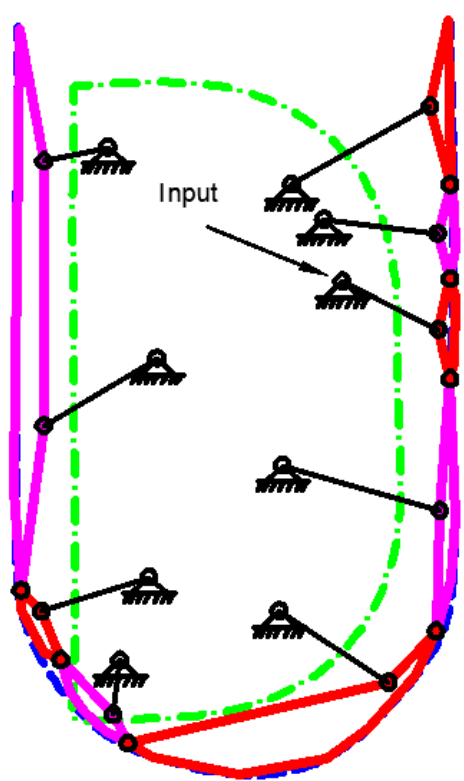

(a)

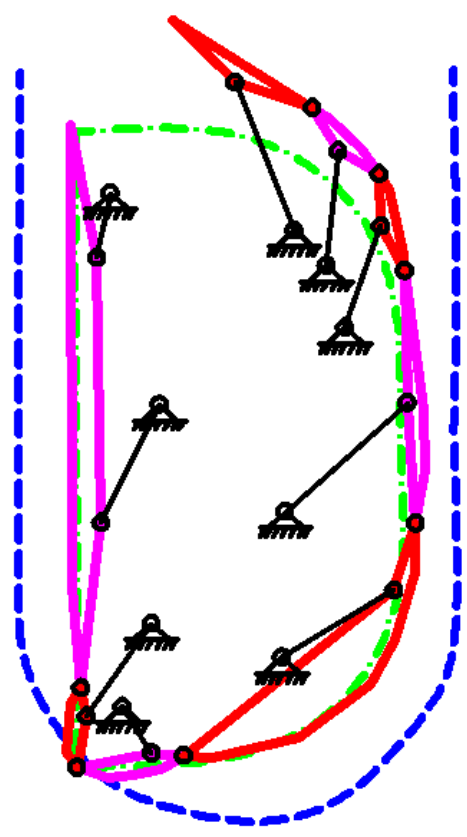

(c)

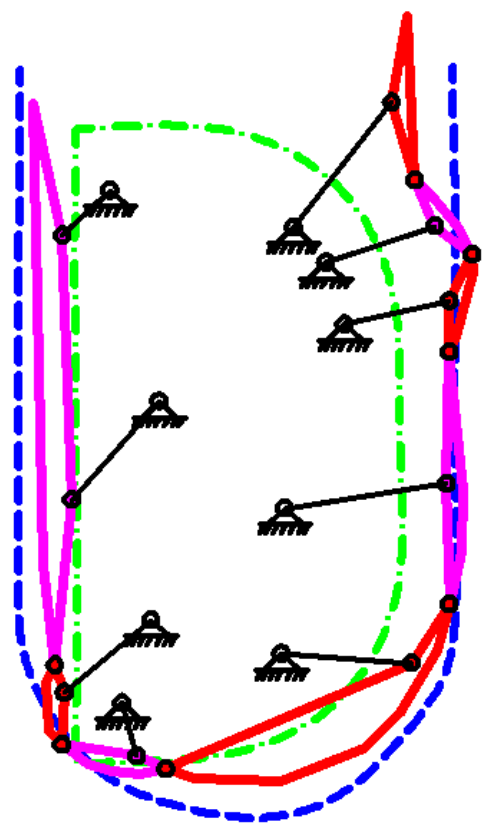

(b)

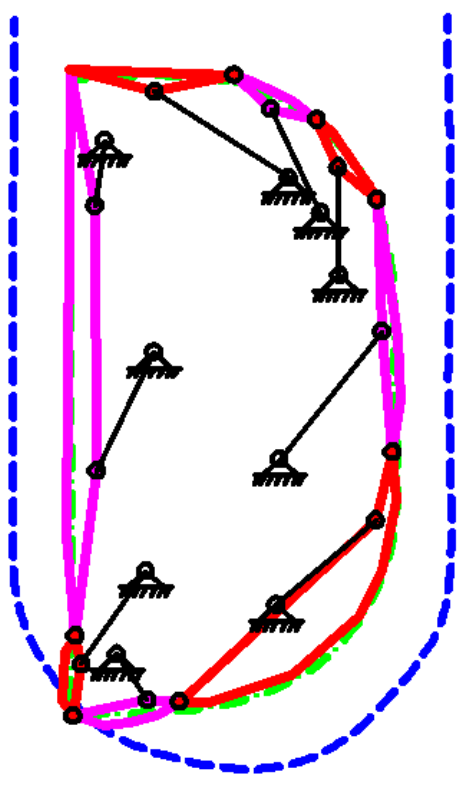

(d)

Figure 11: The mechanism is capable of moving the chain from approximating the " $U$ " to the " $D$ " as it progresses from (a) through (d) based on work in [46]. 
and design theories, rigid-body mechanisms readily produce large displacements while delivering high load-carrying capacities. Potential drawbacks include backlash, wear, increase in part-count, weight, assembly cost and time, and routine maintenance [48]. As rigid-body mechanisms are common and encounter significant use, these drawbacks are well addressed in most machine designs $[49,50]$.

In this work, the rigid-body shape-changing mechanisms are composed of bodies connected by revolute $(\mathrm{R})$ and prismatic $(\mathrm{P})$ joints. Examples of rigid-body shape-changing designs from Section 1.1 include the morphing wing in Fig. 2 and morphing car spoiler in Fig. 3, the convertible car roof in Fig. 7, the mobile performance stage in Fig. 8, and the artwork example in Fig. 11. As the complexity of some of these devices may indicate, an additional design challenge in several of these examples is the identification of layering of the links in order to avoid interference [51, 52].

Generally, rigid-body mechanisms can be driven with a single actuator [14]. The input force or torque can be applied to one of the links of the mechanism [53]. Actuation of rigid-body mechanisms is typically achieved with various kinds of electric motors, ball screws, hydraulic and pneumatic cylinders, springs and elastic bands, and occasionally, human-power [14]. Electric motors like AC motors, DC motors, geared motors, stepper motors, and servomotors provide rotary motion that can be attached to a driving axle. Some motors are integrated with ball screw mechanisms to provide linear motion. Hydraulic and pneumatic systems use hydraulic oil and air respectively to initiate linear motion in cylinders. Manual actuation by cranking and levering also see common use in many mechanisms. Nonetheless, output motions can also be achieved by having multiple actuators such as in many industrial robots. Kota and Erdman [54] argued that this is usually not an efficient use of energy and should be considered primarily if flexibility in motion is required. In addition to the widely-used actuators mentioned previously, mechanisms can be actuated by shape-memory alloys [9] or piezoelectric materials [55, 56, 57]. 
Due to their well established principles and components, rigid-body mechanisms offer a solution worthy of consideration in solving shape-change problems. Rigid-body shape-changing mechanisms have the potential to benefit from qualities such as high stiffness and large load carrying and displacement capabilities.

\subsubsection{Compliant Mechanisms}

Shape-change may be accomplished by using compliant mechanisms, which can be designed in a manner similar to rigid-body mechanisms [58]. Without hinges, though, compliant mechanisms have the advantage of providing a smooth morphing boundary without discontinuities. Compliant mechanisms usually have qualities of both a mechanism and a structure [34]. Howell introduced the concept of the pseudo-rigid-body model to aid the design of compliant mechanisms [47]. Another method used in their synthesis is topology optimization, which attempts to solve a design problem by iteratively removing material from regions in a structure that are less sensitive to reducing the overall structural stiffness and thus change its final shape [33, 34].

Section 1.1 lists many compliant shape-changing devices including a prosthetic hand, morphing aircraft wing in Fig.1, and robotic gripper as in Fig. 9. Shuib et al. [48] show other applications including compliant staplers and knee-joints. Additionally, there are also many biologically inspired technologies including those for flapping wings [59] and artificial biomimetic robotic swimming fish [60].

Goldfarb and Speich [61] mention that a benefit of compliant mechanisms is the absence of revolute joints and bearings, thus eliminating mechanical backlash (from clearance) and Coulomb friction (which causes wear). Parlaktas [62] added other potential advantages of compliant mechanisms including low cost, reduced number of parts, reduced weight, no requirement for lubrication, 
and less noise. Mankame and Ananthasuresh [63] note that compliant mechanisms have the advantages of being a single-piece and utilizing elastic deformation of the mechanism bodies instead of kinematic joints, which is very useful at the micro level since they do not require assembly. Compliant mechanisms also store elastic energy as they deform, so there is no need for springs to return the mechanism to its original position. Consequently, this also means they require a holding force in a deformed position.

Other disadvantages of compliant mechanisms include the relatively small range of motion, imprecise axis-drift motion, off-axis stiffness, and high stress-concentration at the joints [61, 64]. Converting reciprocating translation to rotational motion is also a problem that requires alternative approaches such as a contact-aided compliant mechanism [63]. The displacement achievable with a compliant mechanism is limited by the elastic properties of its composing material, so it is quite difficult to accommodate shape changes involving significant differences in arc length.

\subsubsection{Membranes and Smart Materials}

In space, a very large, deployable telescope can be made of segments of stretched reflective membranes [37,38]. Being lightweight and flexible, they can be transported in a compact configuration. Section 1.1.3 included a coated deformable membrane mirror that changes shape significantly when deployed [17, 32, 39]. Typically, after a mirror is deployed, the flatness of its flexible surfaces requires correction. This may be accomplished via the use of electrostatic, electromagnetic, electron gun or boundary actuation techniques in a closed-loop system [38, 65].

In another space application, Otsuka et al. [66] showcased a nickel-titanium alloy for a space antenna as shown in Fig. 18. Smart materials typically change shape via an applied voltage, when heated, or due to changes in magnetic force, humidity, or $\mathrm{pH}$ value $[67,68,69,70]$. They can achieve a finite number of distinct shapes. Zanaboni [71] explained that one type of smart material 
is the shape-memory alloy (SMA), which may be classified as "one-way" or "two-way". The oneway materials may be deformed to change shape at low temperature. When heated above a certain temperature, they start to revert to their original shape in the austenitic phase. A two-way SMA material can assume two pre-trained shapes by certain thermomechanical treatments that depend on heating or cooling at certain temperatures. This represents a limitation should there be more shapes to be approximated. Research in wings and helicopter blades that utilize smart materials typically incorporate piezoceramics or active fiber composites such as in Weisshaar et al. [10, 11], Cesnik et al. [16], Wong [17], and Grohmann et al. [18].

\subsubsection{Combinations Involving Membranes and Rigid Bodies}

Many systems considered for shape change require smooth design profiles. However, rigid-body rotation about discrete revolute joints will result in a profile with abrupt continuity changes that may hinder the approximation of a smooth curve. Recent advancements in membrane technology for mirrors, aeronautics, and automotives could be coupled with rigid-body mechanisms to address this issue $[72,57]$. For instance, BMW is investigating shape-changing cars that are covered with plastic-coated lycra fabric that is stretched over a metal frame with moving components. This concept car, GINA [73], is shown in Fig. 12.

\subsection{Rigid-Body, Shape-Changing, Mechanism Design Methodology}

This section overviews the established design process for planar rigid-body shape-changing mechanisms that includes design profile specification, target profile generation, segmentation, and mechanization. 


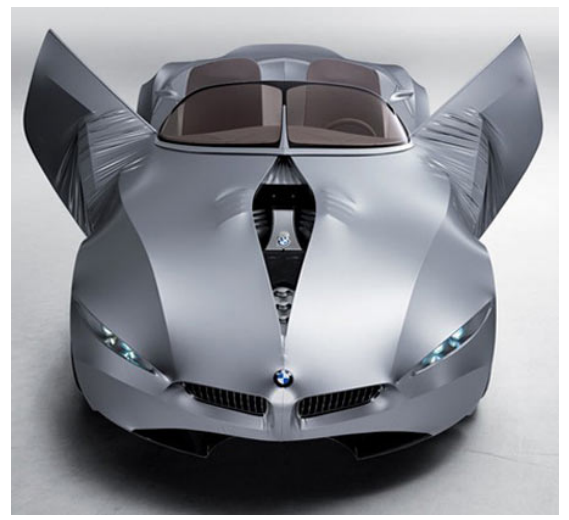

Figure 12: The "skin" or outer layer of the shape-changing machine does not have to be rigid. The GINA has the skeleton mechanism underneath made of rigid members [73].

\subsubsection{Profiles and Segmentation}

Planar rigid-body shape-changing mechanism design begins with a set of design profiles, such as airfoil profiles for loiter and attack modes. The synthesis process proceeds by representing each of the design profiles in a standardized manner, such that comparisons can be made among them. This standardized representation is a coordinated set of points on the design profiles defining a piecewiselinear curve that is termed the target profile. The design process continues with a segmentation phase that creates segments, which are generated in shape and length so that they form a jointed chain of rigid bodies that approximate corresponding segments on each target profile. To complete the synthesis, a mechanization phase typically adds binary links to each segment in order to achieve the lowest degree-of-freedom (DOF) linkage possible. Although this established process of designing a rigid-body shape-changing mechanism remains true for this work, substantial changes are needed to allow for significant differences in arc length. 


\subsubsection{Mechanization Methods}

A system with a low DOF is commonly preferred for simplicity in control $[74,75]$. The mechanization phase involves adding rigid constraining links and joints, forming a mechanism that smoothly transitions the shape approximating chain between the target profiles with a limited number of actuators. In many applications, the reduced cost and control requirements of fewer actuators outweighs the kinematic complexity. When a single-DOF system is desired and the number of target profiles is less than or equal to five, it is theoretically possible to add binary links without further increasing the profile matching error. The dimensional synthesis task for rigid body guidance identifies appropriate circle points on the rigid links of the shape approximating chain and center points on the frame. Machine theory texts, such as McCarthy [76], provide methods for dimensional synthesis for rigid body guidance. However, it was revealed through experience that eliminating circuit, branch and order defects becomes problematic when more than three profiles are involved. Balli and Chand [77] provided a thorough discussion on solution rectification. Consequently, for two and three profiles, the mechanization of shape-changing linkages has been accomplished by adapting dimensional synthesis techniques to Geometric Constraint Programming (GCP) with a computer-aided design package as in Kinzel et al. [78].

For greater than three profiles, mechanization of rigid-body shape-changers is performed as outlined in Murray et al. [46, 79]. Least-squares approximations such as those developed by Yao and Angeles [80] can be used to locate circle and center points for each segment. The structural error associated with such approximate motion synthesis methods will further increase the shape approximating error. A search algorithm is implemented to examine many circle and center point pairs, designating candidate designs as those that produce an acceptable level of structural error. The candidate designs are then evaluated to determine whether they can be actuated monotonically to perform the shape change without encountering a circuit or branch defect. Successful designs 
can be ranked by a quality factor of the designer's choosing. This search approach does not yield optimal designs in any formal sense, but produces a number of viable designs that can be evaluated according to various metrics. Expanding on the search process, Zhao et al. [81] illustrate how genetic algorithms can be used to synthesize planar rigid-body shape-changing mechanisms. Once a successful mechanism has been formed, it may benefit from the addition of a coupler driver to reduce actuator effort and avoid mechanism defects [82].

The mobility of a final design is not necessarily restricted to a single DOF or being driven by linkages formed from rigid bodies. There are many other possibilities of actuating a chain of rigidbodies from one position to another. Cable and spring mechanisms [83, 84], smart-material actuators like piezoceramics [71], and multiple-motor actuation [54] can all be considered. Figure 13 shows a segmented prosthetic finger that can bend into a curled shape by tensioning its cable [83]. Alternately, robotic systems are typically serial links actuated at every joint. This dissertation does not focus on the mechanization stage of the work and an ad-hoc method is used to complete several examples in Chapter V. Regardless of the fact that a mechanization is shown, the chain may be controlled in a variety of ways with additional consideration given to the appropriate degrees of freedom.

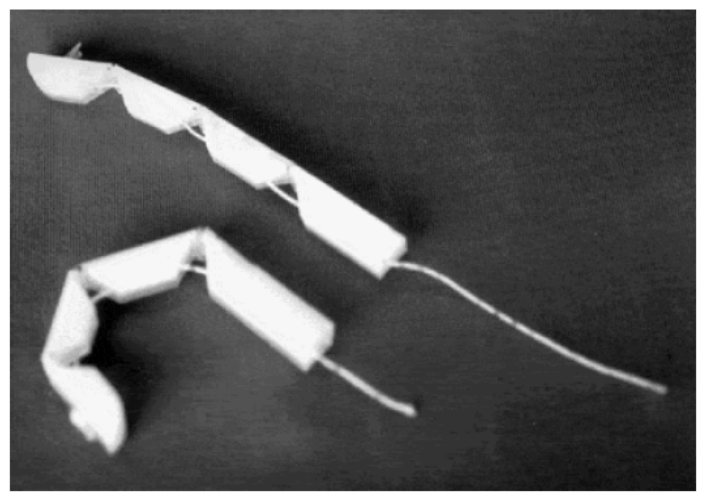

Figure 13: A cable extends the length of the prosthetic finger and acts as an actuator [83]. 


\subsubsection{Limitations of This Method}

When two profiles are not similar in arc length, the resulting mean profile does not approximate either of the two profiles well. This, however, depends on the significance of the difference in arc lengths. Figure 14a illustrates the mean segment generated for points 1 through 3 of the profiles, whereas Fig. 14b shows two copies of the mean segments being placed on top of the two original segments. Obviously, the mean segments do not approximate any portion accurately. Another problem is that the method is not capable of assigning a segment type that can change length. Hence, there is a need for another method to solve different arc-length problems.

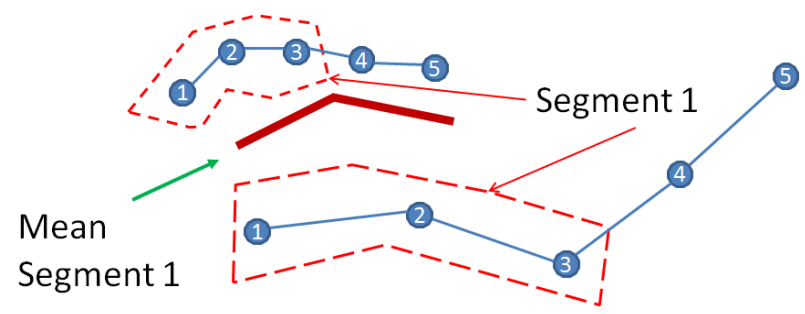

(a)

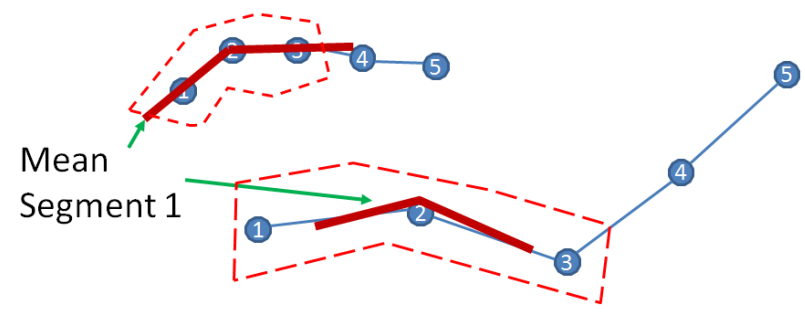

(b)

Figure 14: (a) A mean segment generated for points 1-2-3 of the two different length profiles. (b) Mapping the mean segments on the profiles does not show good results. 


\subsection{Shape-Changing for Different Arc-Length Profiles}

Some examples of shape-changing mechanisms that have different arc-length profiles include wings, boat hulls, satellite antenna, and forming dies. This dissertation proposes new methodologies for the design of rigid-body shape-changing mechanisms that approximate design profiles with significant differences in arc length. This affects both the profile generation and segmentation phases.

\subsubsection{Different Arc Length Examples}

Many large aircraft wings have multiple elements to achieve the required lift and drag for different flight modes. When in operation, some of these elements are deployed to effectively extend the profile of the wings. Figure 15 shows a pressure distribution plot for a four-element wing [85], which is an example of different arc length profile for a shape-changing mechanism.

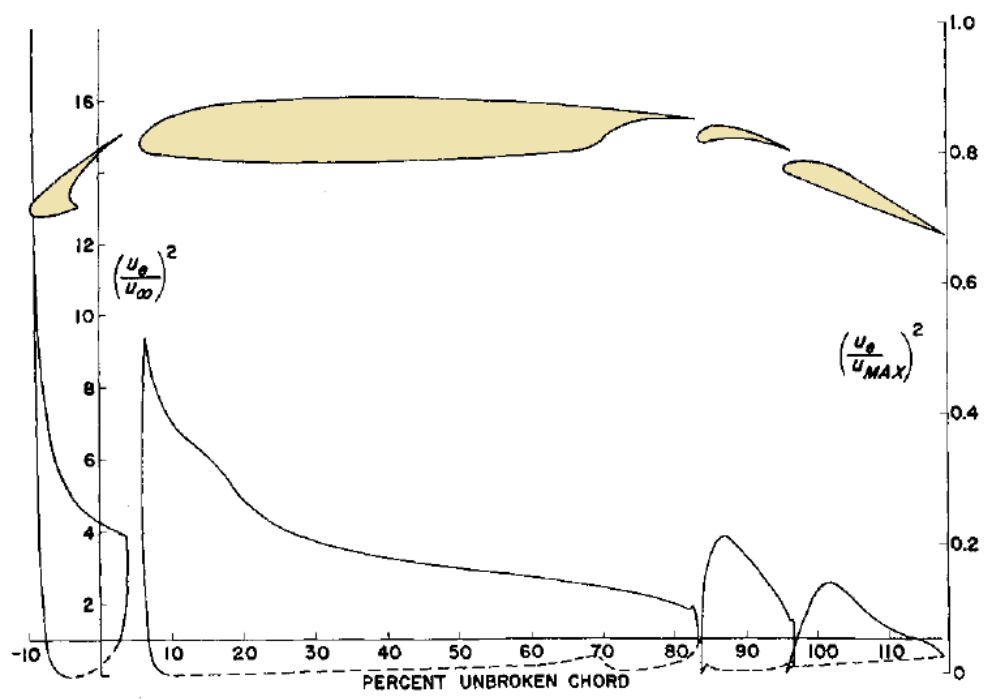

Figure 15: A conventional theoretical pressure distribution $C_{p}$ plot of a four-element wing. The leading-edge and trailing-edge flaps are in deployed positions. 
Wong [17] highlights the idea of a shape-changing boat hull that can affect maximum speed, ride comfort, and fuel consumption. Several basic foil shapes used by naval designers are shown in Fig. 16. These also show the need for different arc-length shape-changing mechanisms since the profiles' perimeter are significantly dissimilar. Gillmer [26] explained that modern ship designs employ modifications of these throughout sections of the hull as illustrated in Fig. 17. While overly ambitious for large sea-going ships, a shape-changing hull is an attractive concept that would enable much smaller boats to make adjustments for the navigation of seas, rivers, lakes, swamps etc.

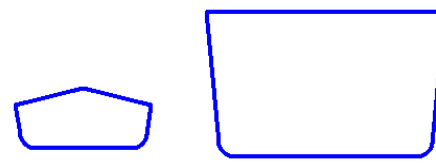

(a) Planing (b) Deep hull

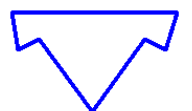

(c) Multi hull

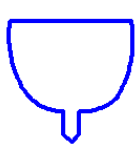

(d) Round hull

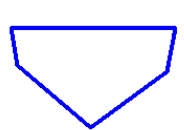

(e) Deep vee

Figure 16: The various types of hulls for ships and boats.

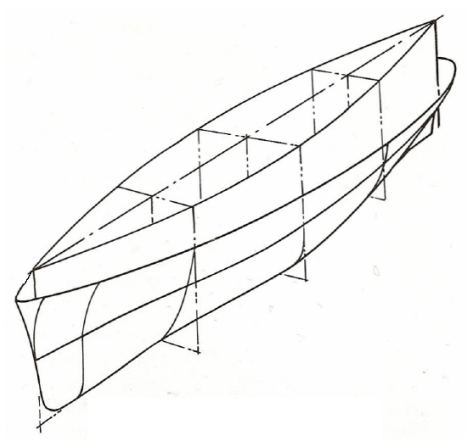

Figure 17: Different cross-sectional shapes along a modern ship's hull [26].

Goodyear Aerospace Corporation studied the use of Ti-Ni shape-memory effect (SME) wires in a shape-changing antenna [66]. This requires a different arc-length mechanism, probably in a 
spatial environment. Figure 18 shows the progression as the deformed wires revert back to the original shape of the antenna through solar heating.

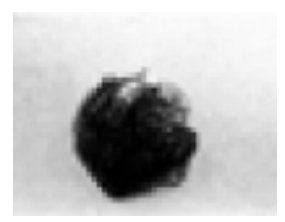

(a) Deformed shape

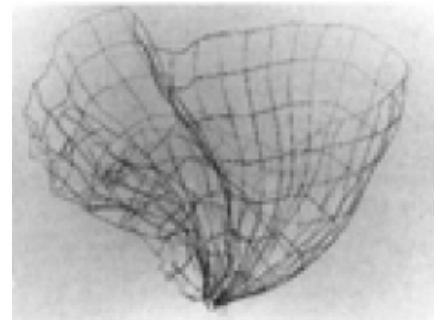

(c) Interim shape 2

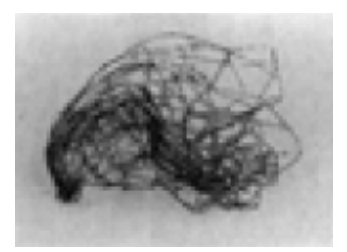

(b) Interim shape 1

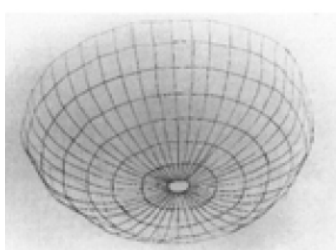

(d) Fully deployed shape

Figure 18: The Ti-Ni wires return to its original antenna shape by heating [66].

Altering an extruded plastic profile by sliding segments of the die orifice in a controlled manner [86] may also be considered a shape change. Choi et al. [87] investigated the forming of aluminum frames for automotive applications through the use of a $\mathrm{CNC}$ variable section extruder. Figure 19 shows the schematic diagram of their experimental setup and the finished product that has different cross-sections at opposite ends. Note that the perimeters of the sections exhibit drastically different arc lengths. O'Connor proposes a method to produce a pultruded thermoplastic composite body having at least two integral sections of different cross-sectional shapes [88]. The system arranges different sets of dies in series as illustrated in Fig. 20a. Potential varying cross-section pultrusions are shown in Fig. 20b. 


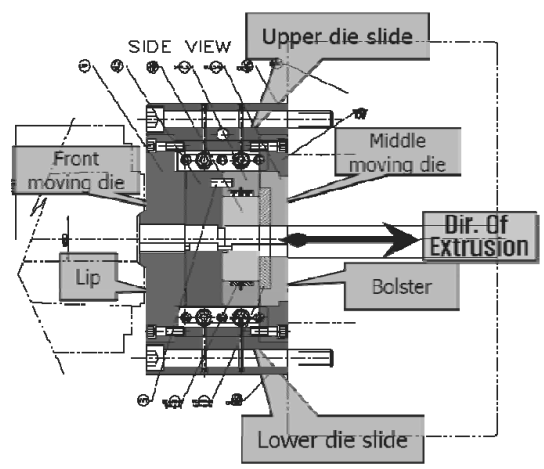

(a)

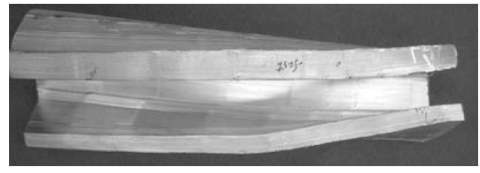

(b)

Figure 19: (a) Schematic diagram of the multiple dies extrusion system. (b) The frame is axially twisted, and the width of the cross section varies steadily [87].

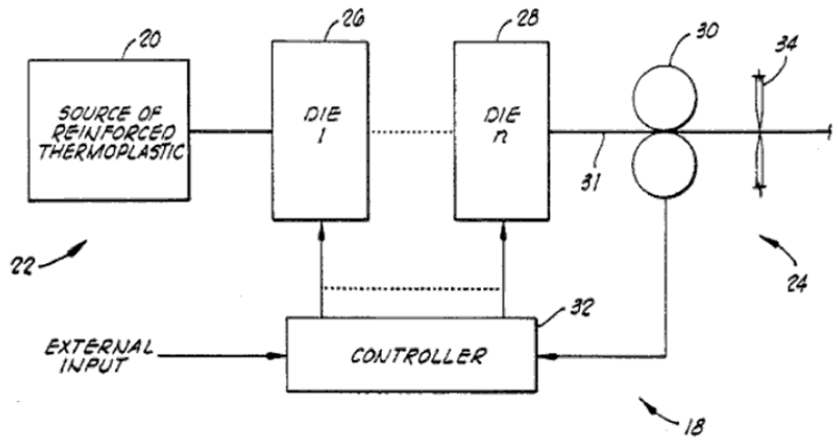

(a)

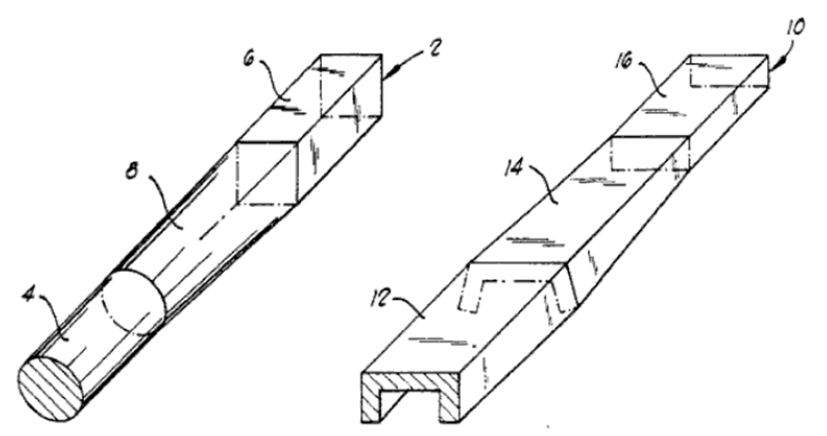

(b)

Figure 20: (a) Schematic diagram of the multiple dies pultrusion system. (b) The cross-sectional shapes vary gradually [88]. 
Murray et al. studied the design of a shape-changing extrusion or pultrusion die [89]. Different arc-length profiles can be seen in Fig. 21a and the extrusion machine that is designed to produce such shapes is shown in Fig. 21b.

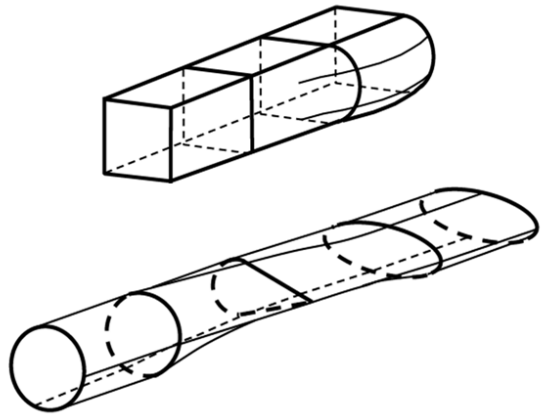

(a)

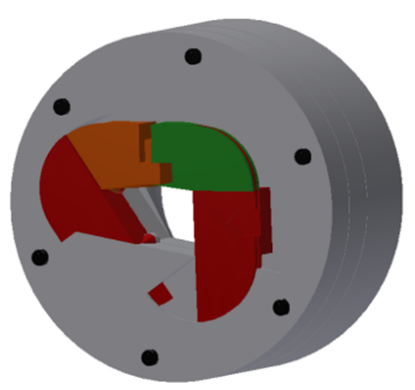

(b)

Figure 21: (a) Various cross-section shapes for the two extruded products. (b) The shape-changing die concept [89].

\subsubsection{New Profile Generation}

The curves that are specified as the desired shapes to be matched by the edge geometry of rigid links are called design profiles. Points are generated along each design profile that when connected by line segments, approximate the design profiles. These new curves are called the target profiles. The line segment connecting two adjacent points is called a piece. Correspondingly, the number of pieces will be one less than the number of points on each target profile. Since profiles can have different arc lengths, the number of points used may not be identical from one design profile to another. Murray et al. [46] assign the same number of points to each target profile. With significant differences in arc length, this work assigns a different number of points to each profile, distributed such that each piece length is approximately equal to a designer-specified piece length. This is to 
allow for the inclusion of rigid bodies connected with prismatic joints, which can vary the length. As the desired piece length is made smaller, the generated profiles better approximate the shapes of the design profiles.

\subsubsection{New Segmentation Method}

Segmentation is the second phase of the design process. The goal of this phase is to create segments that are generated in shape and length so as to form rigid links that approximate corresponding segments on each target profile. Connecting these segments with revolute joints yields a chain of bodies that approximates the target profiles and hence, the design profiles. There are two basic types of segments, the mean segment ( $\mathscr{M}$-segment) and the constant curvature segment ( $\mathscr{C}$-segment).

When a segment contains the same number of points on each target profile, an $\mathscr{M}$-segment is generated. The segments are transformed to the corresponding segment on a reference profile in distance minimizing configurations. The $\mathscr{M}$-segment is generated as the geometric center of each set of corresponding points before being transformed back to approximate the corresponding segments. A poor match may be improved by reducing the number of points being matched or changing start and end points.

When a different number of points on each target profile are to be approximated, a $\mathscr{C}$-segment is generated. By calculating an average radius of constant curvature and an average piece length, a segment of constant curvature is generated. As with the $\mathscr{M}$-segment, a poor match may be improved by reducing the number of points being matched or changing start and end points.

$\mathscr{M}$ - and $\mathscr{C}$-segments that approximate all pieces on all target profiles are assembled by joining their end points with revolute joints. These assembled chains are repositioned to best match each target profile to gauge accuracy. If accuracy is lower than the application demands, the order of $\mathscr{M}$ - 
and $\mathscr{C}$-segments may be changed, the length of each segment may be changed, or the number of segments may be increased.

In addition to the two basic types of segments, a third type of segment may be formed by fusing an $\mathscr{M}$ - and a $\mathscr{C}$-segment or two $\mathscr{C}$-segments. Segments are fused after they are joined as a chain and aligned with the profiles. Should the relative rotation about an $\mathrm{R}$ joint be small, fusing of the segments is considered. Note that $\mathscr{M}$-segments are not fused with other $\mathscr{M}$-segments.

\subsection{Organization}

With the goal of segmentation of a set of design profiles of significantly different arc length into a chain of rigid-bodies connected by $\mathrm{R}$ and $\mathrm{P}$ joints, this dissertation is organized as follows. Chapter II discusses the creation of target profiles when the arc lengths of the design profiles are significantly different. Having created the target profiles, Chapter III explains the generation of $\mathscr{M}$ - and $\mathscr{C}$-segments. The MATLAB software implementation that enables the design of segments to be carried out is explained in Chapter IV. Chapter V discusses several mechanization examples in which different profiles serve to show the utility of the methods developed in this work. Lastly, conclusions and suggestions for future work are furnished in Chapter VI. A user's guide in Appendix A provides examples of how to use the software to create a chain of jointed segments from a set of design profiles. 


\section{CHAPTER II}

\section{OPERATIONS ON TARGET PROFILES}

The first goal of this chapter is to showcase the process for creating target profiles from a set of design profiles. Design profiles specify the design challenge in that they are the set of curves used to describe the shapes that the shape-changing mechanism should approximate. Target profiles are the set of curves generated from the design profiles but defined by points spaced to facilitate approximation by a connected chain of rigid links. Once generated, target profiles replace the design profiles and are used in all following processes to create the chain of links. The second goal of this chapter is to establish the metrics by which distance between target profiles or their segments can be determined.

\subsection{Target Profile Generation}

The challenge posed by shape-change is the specification of a set of $p$ design profiles that represent the different shapes to be attained by the mechanism. Murray et al. [46] define a design profile $j$ as an ordered set of $n_{j}$ points for which the arc length between any two can be determined. Figure 22 exhibits the three types of design profiles considered: open, closed, and fixed-end profiles. While the illustrative examples shown in this work are for the open profile case, this research establishes a process to form a chain of rigid links to approximate any of these types of profiles. 


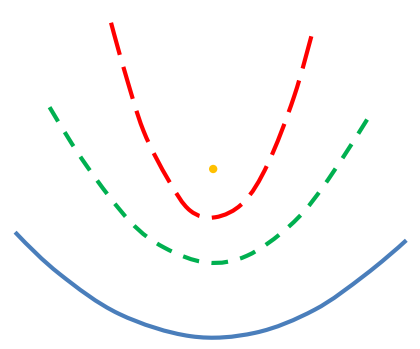

(a)

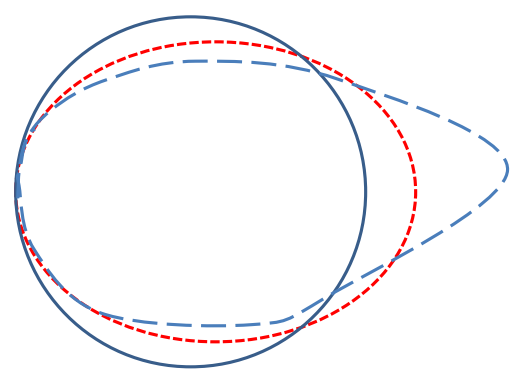

(b)

(c)

Figure 22: Profile types include (a) open profiles, (b) closed profiles, and (c) fixed-end profiles.

Given the definition of design profiles, they may be viewed as being piecewise linear. A piece is the line segment connecting two contiguous points on a profile. The $i^{\text {th }}$ point on the $j^{\text {th }}$ design profile is designated $\left\{a_{j_{i}}, b_{j_{i}}\right\}^{T}$. The length of the $i^{t h}$ piece on the $j^{\text {th }}$ design profile is

$$
c_{j_{i}}=\sqrt{\left(a_{j_{i+1}}-a_{j_{i}}\right)^{2}+\left(b_{j_{i+1}}-b_{j_{i}}\right)^{2}}
$$

and the arc length of the entire $j^{\text {th }}$ design profile is

$$
C_{j}=\sum_{i=1}^{n_{j}-1} c_{j_{i}} .
$$

The design profiles may be defined by any number of points spaced at various intervals, producing a wide range of $c_{j_{i}}$. 
As previously defined, target profiles are a set of curves that represent the set of design profiles. They have common features so that groups of contiguous points can be compared to all profiles in order to form a suitable chain of rigid bodies that when repositioned will approximate all design profiles. In earlier work [46, 79], the design profiles were assumed to be of roughly equal arc lengths, $C_{1} \approx C_{2} \approx \ldots \approx C_{p}$, and each target profile can be formed by distributing the same $n$ number of defining points equally along the corresponding design profile. The target profile becomes a piecewise linear curve composed of pieces with roughly the same length, $c_{j_{i}} \approx c_{k_{l}}$, $\forall i, j, k, l$. Constant piece lengths allow for identification of corresponding points on each target profile.

The general profiles discussed in this work may possess substantial differences in arc length. Using the same number of points on different length profiles would result in different piece lengths and contaminate the shape comparisons among groupings of contiguous points. In order to produce a constant piece length, the conversion scheme from design to target profiles must be modified from the established method to allow for a different number of points on each target profile. By specifying a desired piece length $s_{d}$, the number of pieces $m_{j}$ on profile $j$ can be determined. Smaller values of $s_{d}$ will produce more pieces and typically result in smaller variations between the design and target profiles. Experiments have shown as the number of pieces increases, the time it takes to complete the synthesis increases exponentially. Hence, $s_{d}$ needs to be controlled to achieve the desired accuracy in an acceptable computation time. Figure 23 shows the exponential relationship between $s_{d}$ and the computation time done on a machine with Intel $\AA$ Core $^{\mathrm{TM}}$ i5 M540 processor at $2.53 \mathrm{GHz}$ with $4.00 \mathrm{~GB}$ total memory. The usable space, however, is $2.98 \mathrm{~GB}$ and the system is 32-bit. This experiment used the three-profile example with four segments that is shown in Fig. 25. The experiment considers all possible combinations of segment types. 


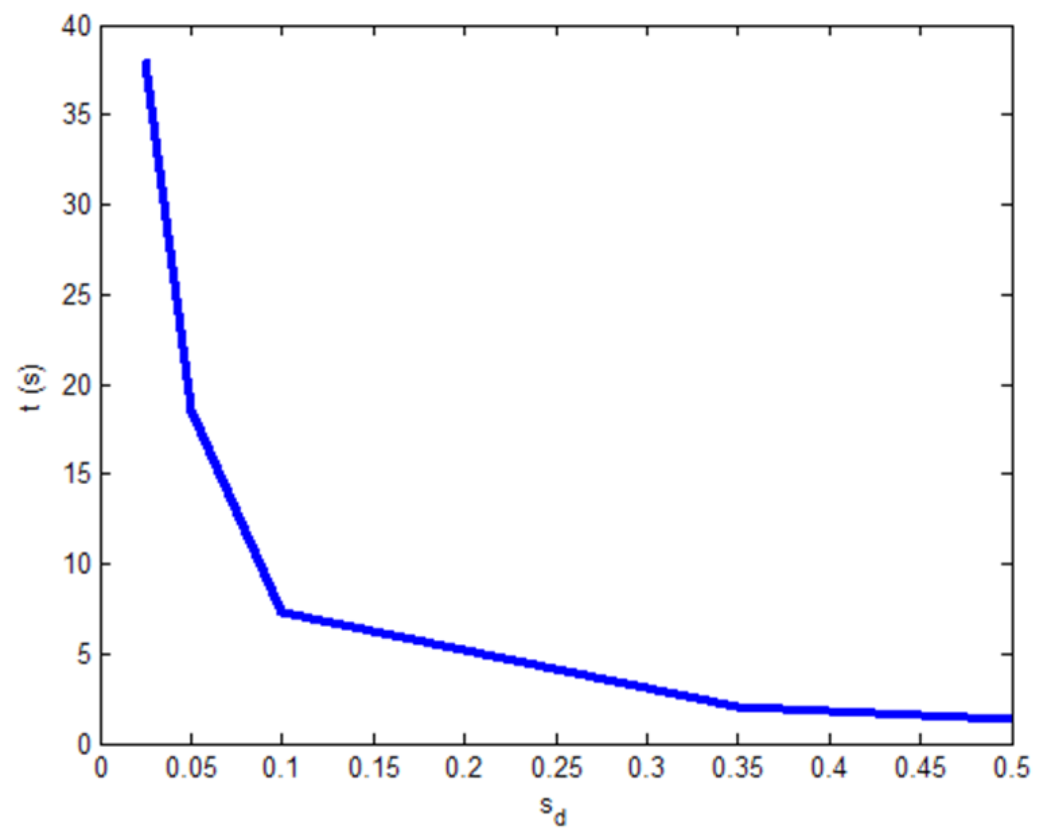

Figure 23: Computation time decreases exponentially as $s_{d}$ increases in size.

The number of pieces must be an integer, and an initial value is calculated as

$$
m_{j}=\left\lceil\frac{C_{j}}{s_{d}}\right\rceil,
$$

where $\lceil\zeta\rceil$ represents the ceiling function, the smallest integer not less than $\zeta$. Provisional target profiles are generated by distributing $n_{j}$ points at increments of $C_{j} / m_{j}$ along the $j^{\text {th }}$ design profile. A distribution of target profile points along a design profile is shown in Fig. 24. The $j^{\text {th }}$ target profile becomes a piecewise linear curve connecting the ordered set of points $\mathbf{z}_{j_{i}}=\left\{x_{j_{i}}, y_{j_{i}}\right\}^{T}$, $i=1, \ldots, n_{j}$. The length of the $i^{t h}$ linear piece on the $j^{\text {th }}$ target profile is

$$
s_{j_{i}}=\left\|\mathbf{z}_{j_{i+1}}-\mathbf{z}_{j_{i}}\right\|=\sqrt{\left(x_{j_{i+1}}-x_{j_{i}}\right)^{2}+\left(y_{j_{i+1}}-y_{j_{i}}\right)^{2}} .
$$

The corresponding number of points on the target profile $j$ is $n_{j}=m_{j}+1$.

For a provisional target profile, the piece lengths $s_{j_{i}} \leq s_{d}$, as Eq. (3) generates slightly more segments than would exactly match the profile using the piece length $s_{d}$. Moreover, any curvature 


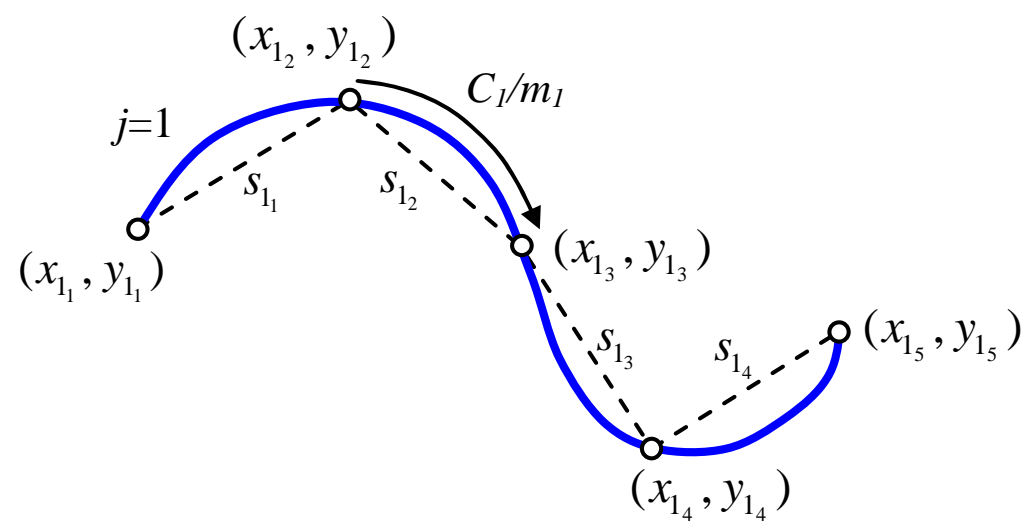

Figure 24: Design profile (solid) with an approximating target profile (dashed) where points are positioned to give a constant arc length along the design profile.

of the design profile results in piece lengths shorter than $s_{d}$, as seen in Fig. 24. That is, some piece $s_{j_{i}}=s_{d}$ only when $s_{d}$ divides exactly into $C_{j}$ and the design profile has a zero curvature portion long enough to include the entire piece. The average piece length for the $j^{\text {th }}$ profile is

$$
\bar{s}_{j}=\frac{1}{m_{j}}\left(\sum_{i=1}^{n_{j}-1} s_{j_{i}}\right) .
$$

As the provisional target profile is constructed to (potentially) have too many pieces to accurately achieve $s_{d}$, the likely scenario is that fewer pieces will produce a value of $\bar{s}_{j}$ closer to $s_{d}$. An error representing the difference between the average segment length and desired piece length is calculated as $\epsilon_{s_{j}}=\left|s_{d}-\bar{s}_{j}\right|$. Decreasing $n_{j}$ by 1 and redistributing points along the design profile creates a new target profile. Points are removed until $n_{j}=n_{j}^{*}$ (and correspondingly, $m_{j}=m_{j}^{*}$ ) that minimizes $\epsilon_{s_{j}}$. The end result is the fewest $n_{j}^{*}$ points are used to construct the $j^{\text {th }}$ target profile such that all linear piece lengths are approximately equal to the desired piece length. Desirable target profiles are those with the fewest pieces that achieve the accuracy needed to satisfactorily represent the original design profiles. The more pieces used in a set of target profiles, the closer the 
approximation of the design profiles. Conversely, as the calculations presented in the later sections are dependent on this number, having fewer pieces reduces computation time.

After each $m_{j}^{*}$ is established, the total length of the $j^{\text {th }}$ target profile is calculated as

$$
S_{j}=\sum_{i=1}^{m_{j}^{*}} s_{j_{i}} .
$$

Applying this process to all design profiles, $p$ target profiles are constructed such that all linear pieces have lengths that are approximately equal to $s_{d}$. The average length of all $m_{j}^{*}$ linear pieces on all $p$ profiles is

$$
\bar{s}_{m}=\frac{\sum_{j=1}^{p} S_{j}}{\sum_{j=1}^{p} m_{j}^{*}} .
$$

If the representation of the design profiles lacks the desired accuracy, a smaller desired piece length may be used to increase the number of points defining the target profiles.

Three target profiles are shown in Fig. 25. The lengths of the design profiles are $C_{1}=26.1738$, $C_{2}=31.0847$, and $C_{3}=34.4737$. For a desired piece length of $s_{d}=0.35$, the target profiles have $m_{1}^{*}=76, m_{2}^{*}=90$, and $m_{3}^{*}=99$ pieces. The average piece length is $\bar{s}_{m}=0.3499$ and the lengths of the target profiles are $S_{1}=26.1669, S_{2}=31.0760$, and $S_{3}=34.4562$. Although $S_{1} / C_{1}=0.9997, S_{2} / C_{2}=0.9997$, and $S_{3} / C_{3}=0.9995$ in this example, the heuristic in this work is to set $s_{d}$ such that $S_{i} / C_{i} \geq 0.99$ for all profiles in the set unless the design problem dictates a specific accuracy. Values are reported in this initial example to four decimal places to highlight the minor differences between design and target profile lengths and desired and achieved piece lengths. The four decimal places are not meant to suggest that these are significant figures.

\subsection{Metrics on Target Profiles}

In the design of rigid-body shape-changing mechanisms, a designer is presented with two (or more) target profiles. To complete the design of the mechanism, an "average" representation of 


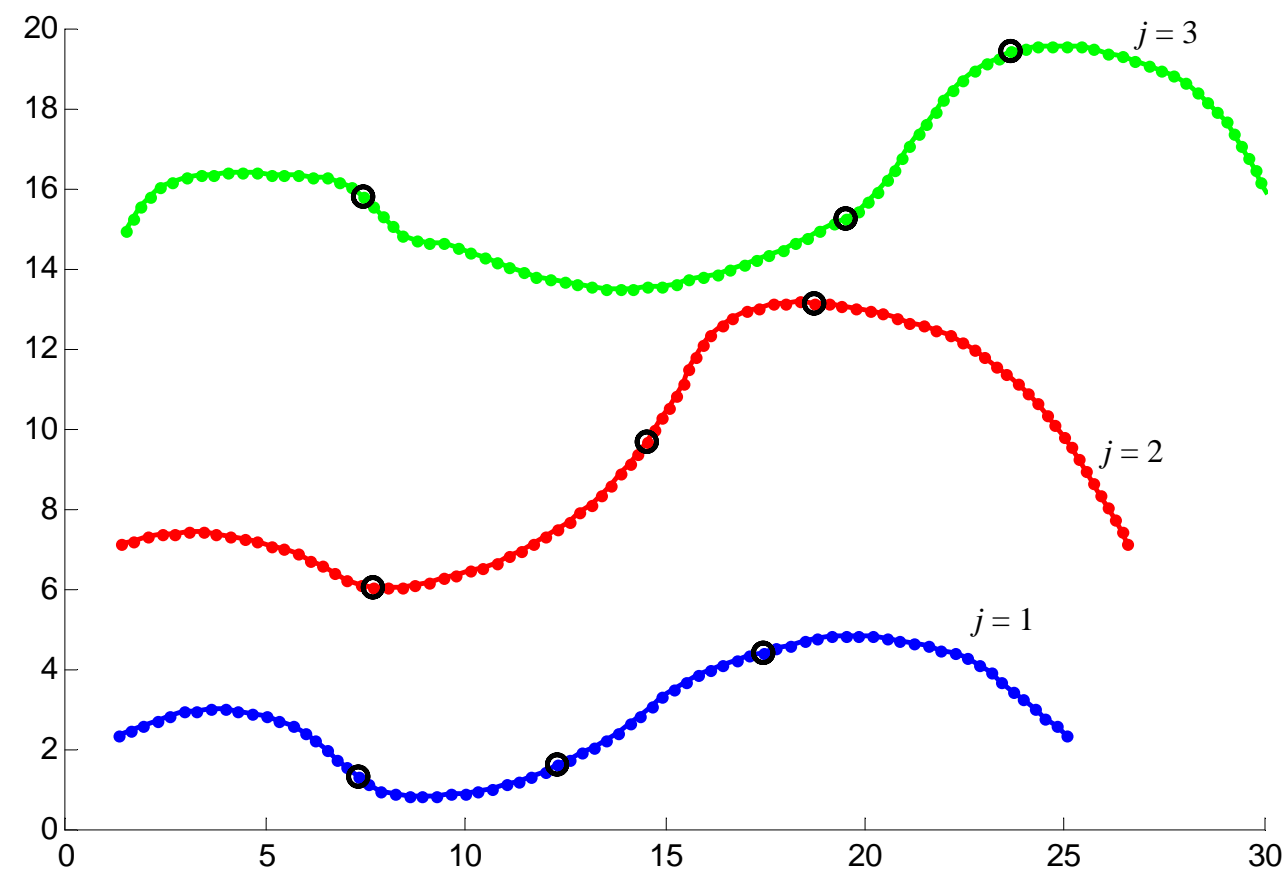

Figure 25: Three design profiles with significantly different arc lengths represented with target profiles of nearly constant piece length.

these profiles is determined. Three such profiles are shown in Fig. 26a. Denoting the points on the "reference profile" as $\mathbf{Z}_{1_{i}}$ and those on one of the other two profiles (the dash-dot profile, for example) as $\mathbf{z}_{j}$, the values of rotation matrix $\mathbf{A}_{j}$ and displacement vector $\mathbf{d}_{j}$ are calculated that solve Eqs. (8) and (9) under the restriction of scale $b=1$. This restriction is necessary due to the consideration of these points as representing rigid bodies. Figure $26 \mathrm{~b}$ shows both of the target profiles aligned with the reference profile in this way. Continuing with the design of a rigid-body shape-changing mechanism, a "mean profile" is generated from the geometric center of each $\mathbf{Z}_{1_{i}}$ and the shifted $\mathbf{z}_{j_{i}}$, as shown in Fig. 26c. Using additional similarity transformations, the mean profile is now shifted to the locations that solve Eqs. (8) and (9) (again with $b=1$ ) relative to each of the target profiles, as shown in Fig. 26d. Thus, similarity transformations are needed both to align 
all of the target profiles in order to create a mean segment and to move this mean segment back to the locations nearest the original target profiles.

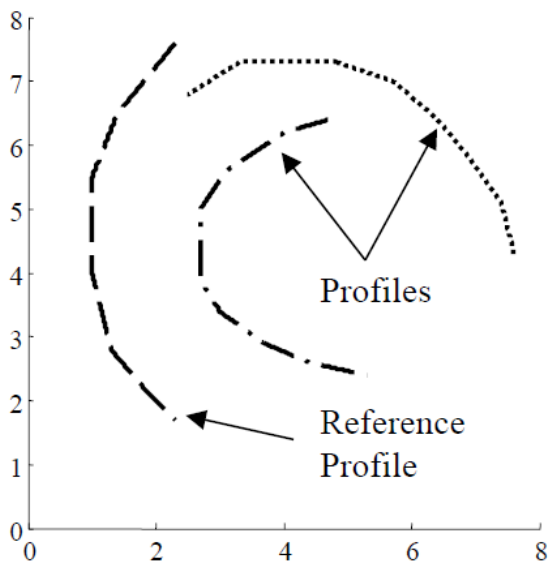

(a)

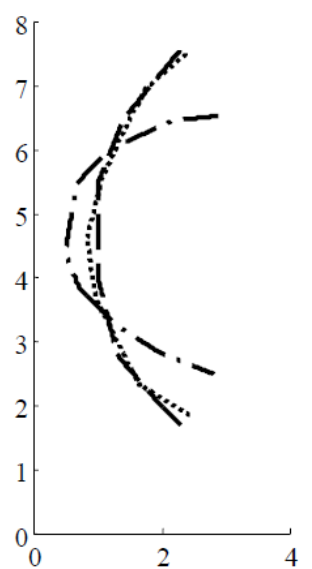

(b)

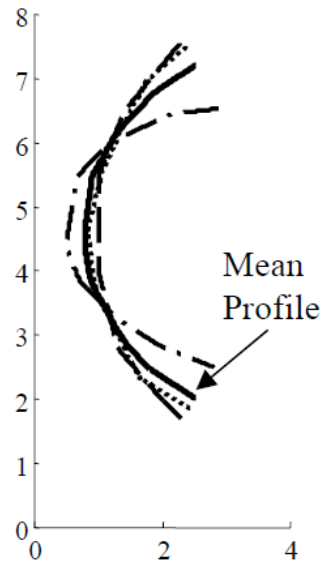

(c)

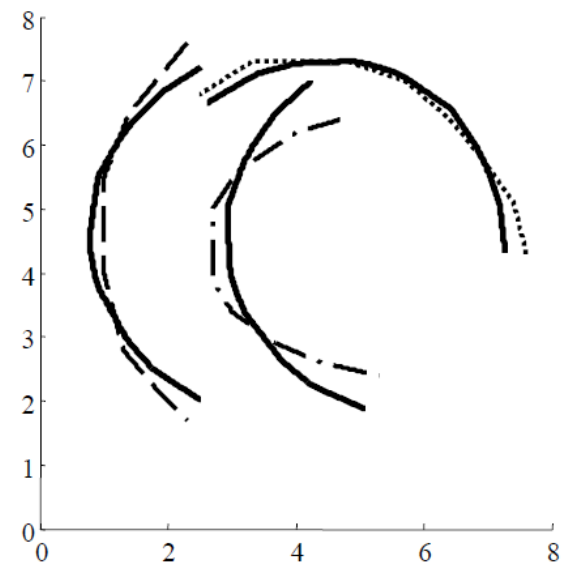

(d)

Figure 26: (a) Three target profiles, with one deemed the reference profile. (b) Two profiles are transformed to the reference by a similarity transformation (with $b=1$ ). (c) The mean profile. (d) The mean profile transformed back to the original profile locations [46].

If the design goal were to find a single body to approximate the three target profiles, the dimensions of a mechanism that guides the body (the mean segment) into the three positions shown 
in Fig. 26d can be determined. Should this fail to reproduce the original profiles with adequate accuracy, the process described above can be performed on individual portions of the target profiles. Figure 27a shows an example in which the three target profiles are considered to consist of four segments. Each segment now does a better job of approximating a shorter section of the original target profile, and combining these segments into a single chain connected by revolute joints produces a more accurate matching of the original target profiles. Figure $27 \mathrm{~b}$ shows the complete mechanism that can be used to move the chain between the three profiles. Note that increasing the number of segments increases the complexity (the number of links) of the mechanism that guides the segments along the three profiles. The details of this process are found in Murray et al. [46] and Persinger et al. [79].

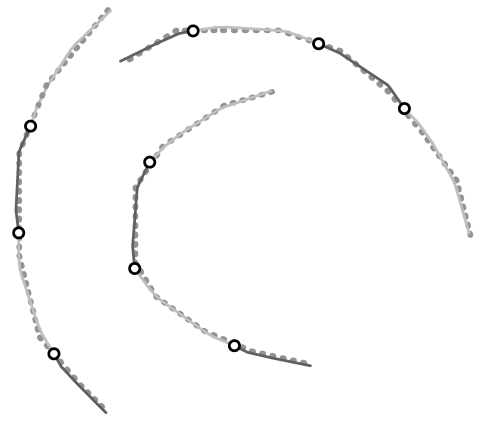

(a) Segmentation

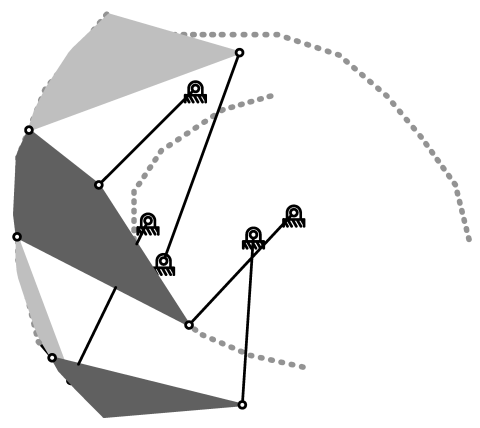

(b) Mechanization

Figure 27: (a) Rigid bodies connected with revolute joints form a chain to closely approximate the profiles. (b) A mechanism design that moves the chain of rigid bodies between the three profiles.

The application of a similarity transformation in mechanism analysis is not far different from the needs in the image registration community. Zitová and Flusser [90] identified a critical step in image registration defined as "transform model estimation." In this step, one attempts to identify the mapping functions that will best align points on a sensed image with the corresponding points on a 
reference image. In planar cases, this mapping function is restricted to be a similarity transformation that can be solved in closed-form as

$$
\mathbf{Z}_{j_{i}}=b \mathbf{A}_{j} \mathbf{z}_{j_{i}}+\mathbf{d}_{j}
$$

where $\mathbf{z}_{j_{i}}$ are the points in the sensed image, $b$ is a scaling factor, $\mathbf{A}_{j}$ is a rotation matrix, and $\mathbf{d}_{j}$ is a displacement vector. The desired values of $b, \mathbf{A}_{j}$, and $\mathbf{d}_{j}$ are those that minimize

$$
D\left(\mathbf{A}_{j}, \mathbf{d}_{j}, b\right)=\frac{1}{N} \sum_{i=1}^{N}\left|\mathbf{Z}_{j_{i}}-\mathbf{Z}_{1_{i}}\right|^{2}, \quad j=2, \ldots, p,
$$

where, for clarity, $\mathbf{Z}_{1_{i}}=\left(Z_{1_{x i}}, Z_{1_{y i}}\right)$ are the points in the reference image and $\mathbf{Z}_{j_{i}}=\left(Z_{j_{x i}}, Z_{j_{y i}}\right)$ are the points in the transformed image.

The solution to Eq. (8) that minimizes $D$ in Eq. (9) has been thoroughly addressed in the relevant literature. Notable among this work is that of Horn [91, 92] and Arun [93] who address the problem for 3-dimensional point sets. Umeyama [94] noted and corrected the problem of an $\mathbf{A}_{j}$ resulting in a reflection (instead of a rotation) for what he refers to as "corrupted data." Both Umeyama's approach and that of Wen [95] work on sets containing points with an arbitrary number of dimensions. Central to the solutions in these papers is the use of the Singular Value Decomposition (SVD). Rapid and robust numerical techniques for determining the SVD are well established in the literature, for example, [96] and [97]. Even more recently, spanning graphs have been proposed due to the efficiency of the associated numerical method [98].

When $\mathbf{Z}_{j_{i}}$ and $\mathbf{z}_{j_{i}}$ are points confined to a plane, though, Eqs. (8) and (9) yield a closed-form solution. Also of note, the correct values of matrix $\mathbf{A}_{j}$, displacement $\mathbf{d}_{j}$, and scale $b$ are generated even in the presence of "corrupted data." Finally, the example from Umeyama [94] is used to verify this methodology. 


\subsubsection{The Closed-Form Similarity Transformation Solution}

The solutions of $b, \theta$ in the rotation matrix

$$
\mathbf{A}_{j}=\left[\begin{array}{cc}
\cos \theta & -\sin \theta \\
\sin \theta & \cos \theta
\end{array}\right]
$$

and the translation vector $\mathbf{d}_{j}$ given by

$$
\mathbf{d}_{j}=\left\{\begin{array}{l}
d_{x} \\
d_{y}
\end{array}\right\}
$$

in Eq. (8) can be found such that

$$
D=\frac{1}{N} \sum_{i=1}^{N}\left|\mathbf{Z}_{j_{i}}-\mathbf{Z}_{1_{i}}\right|^{2}=\frac{1}{N} \sum_{i=1}^{N}\left(\left(Z_{j_{x} i}-Z_{1_{x} i}\right)^{2}+\left(Z_{j_{y} i}-Z_{1_{y} i}\right)^{2}\right)
$$

is minimized. As all summations in Section 2.2 are from $i=1$ to the segment's total number of points $N$, the summation bounds are omitted from all further equations.

The $b, \theta$ and $\mathbf{d}_{j}$ values may be obtained through solving the simultaneous set of equations

$$
\frac{\partial D}{\partial \mathbf{d}_{j}}=\overrightarrow{0}, \frac{\partial D}{\partial \theta}=0, \frac{\partial D}{\partial b}=0
$$

Substituting Eq. (8) into Eq. (12) and expanding gives

$$
\begin{aligned}
D= & \frac{1}{N} \sum\left|b \mathbf{A}_{j} \mathbf{z}_{j_{i}}+\mathbf{d}_{j}-\mathbf{z}_{1_{i}}\right|^{2} \\
= & \frac{1}{N} \sum\left(b^{2}\left(\mathbf{z}_{j_{i}}\right)^{T} \mathbf{z}_{j_{i}}+\mathbf{d}_{j}^{T} \mathbf{d}_{j}+\mathbf{Z}_{1_{i}}^{T} \mathbf{Z}_{1_{i}}\right. \\
& \left.+2 b \mathbf{d}_{j}^{T} \mathbf{A}_{j} \mathbf{z}_{j_{i}}-2 b \mathbf{Z}_{1_{i}}^{T} \mathbf{A}_{j} \mathbf{z}_{j_{i}}-2 \mathbf{d}_{j}^{T} \mathbf{Z}_{1_{i}}\right) .
\end{aligned}
$$

The first of the simultaneous equations in Eq. (13) yields

$$
\frac{\partial D}{\partial \mathbf{d}_{j}}=\sum\left(\mathbf{d}_{j}+b \mathbf{A}_{j} \mathbf{z}_{j_{i}}-\mathbf{z}_{1_{i}}\right)=\overrightarrow{0}
$$

Noting the definitions

$$
\sum \mathbf{z}_{j_{i}}=\mathbf{z}_{j_{t}}=\left\{\begin{array}{ll}
z_{j_{x t}} & z_{j_{y t}}
\end{array}\right\}^{T}
$$


and

$$
\sum \mathbf{z}_{1_{i}}=\mathbf{Z}_{1_{t}}=\left\{Z_{1_{x t}} Z_{1_{y t}}\right\}^{T}
$$

Eq. (15) may be solved for $\mathbf{d}_{j}$ as

$$
\mathbf{d}_{j}=\frac{1}{N}\left(\mathbf{Z}_{1_{t}}-b \mathbf{A}_{j} \mathbf{z}_{j_{t}}\right)
$$

The derivative of $\mathbf{A}_{j}$ is

$$
\frac{\partial \mathbf{A}_{j}}{\partial \theta}=\left[\begin{array}{cc}
-\sin \theta & -\cos \theta \\
\cos \theta & -\sin \theta
\end{array}\right]=\mathbf{A}_{j}\left[\begin{array}{cc}
0 & -1 \\
1 & 0
\end{array}\right]=\mathbf{A}_{j} \hat{I}
$$

and the second of the simultaneous equations in Eq. (13) yields

$$
\frac{\partial D}{\partial \theta}=\sum\left(\mathbf{d}_{j}^{T} \mathbf{A}_{j} \hat{I}_{j_{i}}-\mathbf{Z}_{1_{i}}^{T} \mathbf{A}_{j} \hat{I}_{\mathbf{z}_{i}}\right)=\sum\left(\mathbf{d}_{j}-\mathbf{z}_{1_{i}}\right)^{T} \mathbf{A}_{j} \hat{I} \mathbf{z}_{j_{i}}=0 .
$$

Substituting Eq. (18) into Eq. (20) eliminates $\mathbf{d}_{j}$, and recognizing that as a rotation matrix, $\mathbf{A}_{j}^{T}=\left(\mathbf{A}_{j}\right)^{-1}$,

$$
\begin{aligned}
& \sum\left(\frac{1}{N} \mathbf{z}_{1_{t}}-\frac{1}{N} b \mathbf{A}_{j} \mathbf{z}_{j_{t}}-\mathbf{z}_{1_{i}}\right)^{T} \mathbf{A}_{j} \hat{I} \mathbf{z}_{j_{i}} \\
& =\sum\left(\frac{1}{N} \mathbf{z}_{1_{t}}^{T} \mathbf{A}_{j} \hat{I} \mathbf{z}_{j_{i}}-\frac{1}{N} b \mathbf{z}_{j_{t}}^{T} \hat{I} \mathbf{z}_{j_{i}}-\mathbf{z}_{1_{i}}^{T} \mathbf{A}_{j} \hat{I} \mathbf{z}_{j_{i}}\right)=0 .
\end{aligned}
$$

Observe that

$$
\sum \frac{1}{N} b \mathbf{z}_{j_{t}}^{T} \hat{I} \mathbf{z}_{j_{i}}=\frac{1}{N} b \mathbf{z}_{j_{t}}^{T} \hat{I} \mathbf{z}_{j_{t}}=0
$$

due to the skew-symmetry of $\hat{I}$. Equation (21) becomes

$$
\sum\left(\frac{1}{N} \mathbf{Z}_{j_{i}}^{T} \mathbf{A}_{j} \hat{I} \mathbf{z}_{j_{i}}-\mathbf{z}_{j_{i}}^{T} \mathbf{A}_{j} \hat{I} \mathbf{z}_{1_{i}}\right)=\frac{1}{N} \mathbf{Z}_{j_{t}}^{T} \mathbf{A}_{j} \hat{I} \mathbf{z}_{j_{t}}-\sum \mathbf{z}_{1_{i}}^{T} \mathbf{A}_{j} \hat{I} \mathbf{z}_{j_{i}}=0
$$

Equation (23) can be expanded as

$$
\begin{aligned}
& \frac{1}{N}\left\{\begin{array}{ll}
Z_{1_{x t}} & Z_{1_{y t}}
\end{array}\right\}\left[\begin{array}{cc}
-\sin \theta & -\cos \theta \\
\cos \theta & -\sin \theta
\end{array}\right]\left\{\begin{array}{l}
z_{j_{x t}} \\
z_{j_{y t}}
\end{array}\right\} \\
& =\sum\left\{\begin{array}{ll}
Z_{1_{x i}} & Z_{1_{y i}}
\end{array}\right\}\left[\begin{array}{cc}
-\sin \theta & -\cos \theta \\
\cos \theta & -\sin \theta
\end{array}\right]\left\{\begin{array}{l}
z_{j_{x i}} \\
z_{j_{y i}}
\end{array}\right\} .
\end{aligned}
$$


By expanding Eq. (24) and solving for $\theta$,

$$
\theta=\operatorname{ATAN} 2\left(T_{1}, T_{2}\right)
$$

where

$$
\begin{aligned}
T_{1} & =\frac{1}{N}\left(Z_{1_{x t}} z_{j_{y t}}-z_{j_{x t}} Z_{1_{y t}}\right)-\sum\left(Z_{1_{x i}} z_{j_{y i}}-z_{j_{x i}} Z_{1_{y i}}\right), \\
T_{2} & =\sum\left(Z_{1_{x i}} z_{j_{x i}}+z_{j_{y i}} Z_{1_{y i}}\right)-\frac{1}{N}\left(Z_{1_{x t}} z_{j_{x t}}+z_{j_{y t}} Z_{1_{y t}}\right),
\end{aligned}
$$

and ATAN2 is a four-quadrant inverse tangent function. This determines the proper value of $\theta$, so via Eq. (10), $\mathbf{A}_{j}$ is now known. Since $\mathbf{A}_{j}$ is defined as a rotation matrix, it cannot result in a reflection in the presence of "corrupted data," a possibility when an SVD is used as noted and corrected for by Umeyama [94].

From the third of the simultaneous equations in Eq. (13),

$$
\frac{\partial D}{\partial b}=\frac{1}{N} \sum\left(2 b \mathbf{z}_{j_{i}}^{T} \mathbf{z}_{j_{i}}+2 \mathbf{d}_{j}^{T} \mathbf{A}_{j} \mathbf{z}_{j_{i}}-2 \mathbf{Z}_{1_{i}}^{T} \mathbf{A}_{j} \mathbf{z}_{j_{i}}\right)=0
$$

from which $\mathbf{d}_{j}$ may be eliminated by substituting Eq. (18),

$$
\sum\left(b \mathbf{z}_{j_{i}}^{T} \mathbf{z}_{j_{i}}+\frac{1}{N}\left(\mathbf{Z}_{1_{t}}-b \mathbf{A}_{j} \mathbf{z}_{j_{t}}\right)^{T} \mathbf{A}_{j} \mathbf{z}_{j_{i}}-\mathbf{z}_{1_{i}}^{T} \mathbf{A}_{j} \mathbf{z}_{j_{i}}\right)=0
$$

Expanding,

$$
b\left(\sum \mathbf{z}_{j_{i}}^{T} \mathbf{z}_{j_{i}}-\frac{1}{N} \mathbf{z}_{j_{t}}^{T} \mathbf{z}_{j_{t}}\right)=\sum \mathbf{z}_{1_{i}}^{T} \mathbf{A}_{j} \mathbf{z}_{j_{i}}-\frac{1}{N} \mathbf{z}_{1_{t}}^{T} \mathbf{A}_{j} \mathbf{z}_{j_{t}} .
$$

Recalling that $\mathbf{A}_{j}$ is known, $b$ may be found as

$$
b=\frac{\sum \mathbf{Z}_{1_{i}}^{T} \mathbf{A}_{j} \mathbf{z}_{j_{i}}-\frac{1}{N} \mathbf{Z}_{1_{t}}^{T} \mathbf{A}_{j} \mathbf{z}_{j_{t}}}{\sum \mathbf{z}_{j_{i}}^{T} \mathbf{z}_{j_{i}}-\frac{1}{N} \mathbf{z}_{j_{t}}^{T} \mathbf{z}_{j_{t}}}=\frac{B_{1}-B_{2}}{B_{3}-B_{4}},
$$


where

$$
\begin{aligned}
B_{1}= & \sum\left(Z_{1 x i}\left(z_{j x i} \cos \theta-z_{j y i} \sin \theta\right)\right. \\
& \left.+Z_{1 y i}\left(z_{j x i} \sin \theta+z_{j y i} \cos \theta\right)\right), \\
B_{2}= & \frac{1}{N}\left(Z_{1 x t}\left(z_{j x t} \cos \theta-z_{j y t} \sin \theta\right)\right. \\
& \left.+Z_{1 y t}\left(z_{j x t} \sin \theta+z_{j y t} \cos \theta\right)\right), \\
B_{3}= & \sum\left(z_{j x i}^{2}+z_{j y i}^{2}\right) \\
B_{4}= & \frac{1}{N}\left(z_{j x t}^{2}+z_{j y t}^{2}\right) .
\end{aligned}
$$

Substituting $b$ and $\mathbf{A}_{j}$ into Eq. (18) produces the value of $\mathbf{d}_{j}$, and the similarity transformation is identified.

\subsubsection{Example}

Figures $28 \mathrm{a}$ and $28 \mathrm{~b}$ show the example presented in Umeyama [94] with data that is considered corrupted as the methods established prior to Umeyama yielded a reflection rather than a rotation. The reference points $\mathbf{Z}_{1_{i}}$ are $(0,2),(0,0)$, and $(-1,0)$. The sensed points $\mathbf{z}_{2_{i}}$ to which the similarity transformation is to be applied are $(0,2),(0,0)$, and $(1,0)$. Note that the order of the points is

important as the $N^{t h}$ point of the first list corresponds to the $N^{t h}$ point of the second. Using Eqs. (16) and (17),

$$
\mathbf{z}_{1_{t}}=\left\{\begin{array}{c}
-1 \\
2
\end{array}\right\}, \quad \mathbf{z}_{2_{t}}=\left\{\begin{array}{l}
1 \\
2
\end{array}\right\}
$$

Using Eq. (25), the angle $\theta=-33.69^{\circ}$, and the corresponding rotation matrix is

$$
\mathbf{A}_{2}=\left[\begin{array}{cc}
0.83 & 0.55 \\
-0.55 & 0.83
\end{array}\right]
$$

From Eq. (31), the scaling factor is $b=0.72$. Finally, Eq. (18) is used to find

$$
\mathbf{d}_{2}=\left\{\begin{array}{c}
-0.80 \\
0.40
\end{array}\right\}
$$




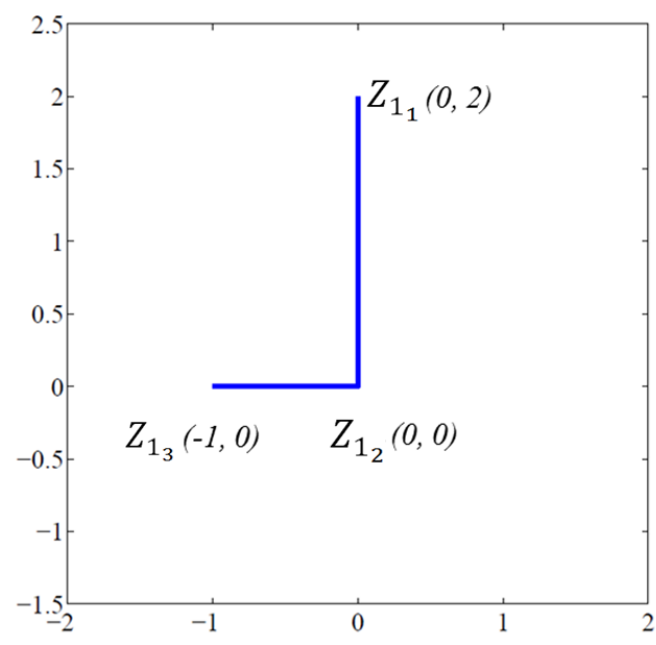

(a)

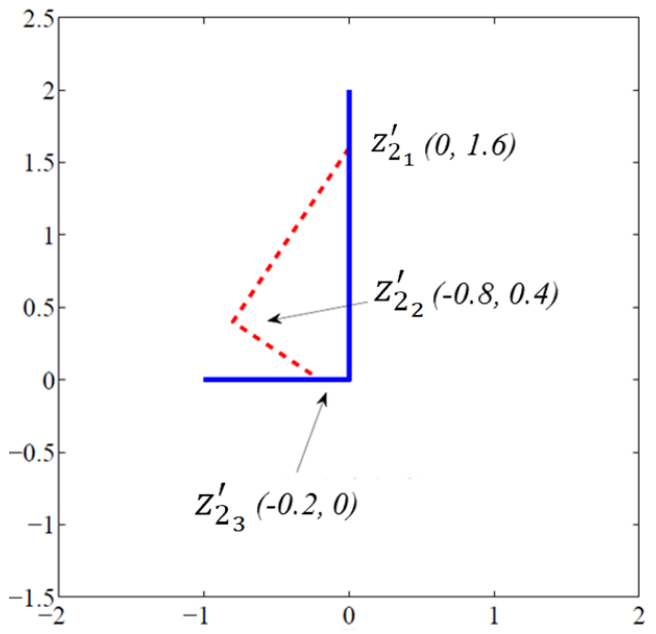

(c)

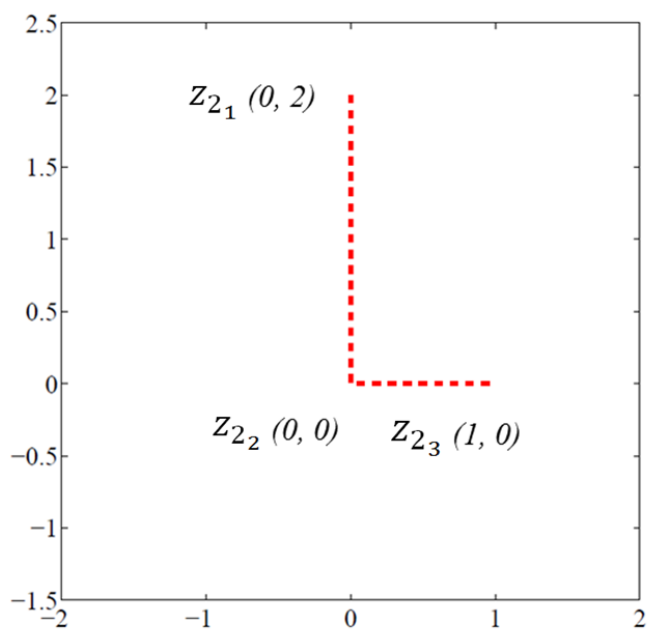

(b)

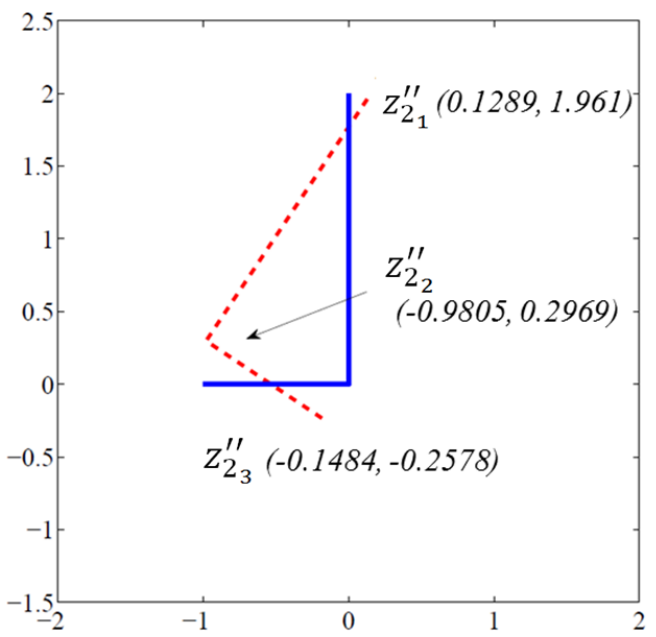

(d)

Figure 28: (a) The reference points $\mathbf{Z}_{1_{i}}$. (b) The points $\mathbf{z}_{j_{i}}$ to undergo the similarity transformation. (c) The scaled, rotated and translated $\mathbf{z}_{2_{i}}$ that align with the $\mathbf{Z}_{1_{i}}$ for the optimal $b=0.72, \theta=$ $-33.69^{\circ}$, and $\mathbf{d}_{j}=[-0.80,0.40]^{T}$. (d) The scaled, rotated and translated $\mathbf{z}_{2_{i}}$ that align with $\mathbf{Z}_{1_{i}}$ for $b=1$. 
These values match those reported by Umeyama [94]. The result of using this similarity transformation to align $\mathbf{z}_{2_{i}}$ with $\mathbf{Z}_{1_{i}}$ is shown in Fig. 28c. Note that for the rigid-body shape-changing calculation, Eq. (31) is not used and $b=1$. In this case, Eq. (18) yields

$$
\mathbf{d}_{2}=\left\{\begin{array}{c}
-0.98 \\
0.30
\end{array}\right\}
$$

The result of using this similarity transformation to align this $\mathbf{z}_{2_{i}}$ with the $\mathbf{Z}_{1_{i}}$ is shown in Fig. 28d.

\subsection{Curvature Calculations}

Determining the curvature at points along a target profile is required to calculate an average curvature over some contiguous set of points. In the next chapter, this average curvature will be used to determine the bend radius in a segment containing a prismatic joint. Curvatures may also be used to compare portions of different target profiles to identify if they admit good approximation by segments of the same constant curvature.

\subsubsection{Calculating the Curvature of Target Profiles}

Excluding the first and last points, the curvature $\kappa_{j_{i}}$ of the $j^{\text {th }}$ target profile at the $i^{t h}$ point can be determined by constructing an arc that passes through $\mathbf{z}_{j_{i}}$ and the neighboring points $\mathbf{z}_{j_{i-1}}$ and $\mathbf{z}_{j_{i+1}}$ [99]. The curvature has a magnitude that is the inverse of the radius passing through the three points,

$$
\kappa_{j_{i}}=\frac{1}{r_{j_{i}}}
$$

The center of a circle passing through $\mathbf{z}_{j_{i-1}}, \mathbf{z}_{j_{i}}$ and $\mathbf{z}_{j_{i+1}}$ is designated as $\left\{\alpha_{j_{i}}, \beta_{j_{i}}\right\}^{T}$, and the general equation of that circle $\left(x-\alpha_{j_{i}}\right)^{2}+\left(y-\beta_{j_{i}}\right)^{2}=r_{j_{i}}^{2}$ can be expanded and rewritten

$$
\left(r_{j_{i}}^{2}-\alpha_{j_{i}}^{2}-\beta_{j_{i}}^{2}\right)+2 x \alpha_{j_{i}}+2 y \beta_{j_{i}}=x^{2}+y^{2}
$$


Substituting the coordinates of the three points into Eq. (38), the three equations can be expressed in matrix form [100],

$$
\left\{\begin{array}{c}
\alpha_{j_{i}} \\
\beta_{j_{i}} \\
\gamma_{j_{i}}
\end{array}\right\}=\left[\begin{array}{ccc}
2 x_{j_{i-1}} & 2 x_{j_{i-1}} & 1 \\
2 x_{j_{i}} & 2 x_{j_{i}} & 1 \\
2 x_{j_{i+1}} & 2 x_{j_{i+1}} & 1
\end{array}\right]^{-1} \quad\left\{\begin{array}{c}
x_{j_{i-1}{ }^{2}+y_{j_{i-1}}{ }^{2}} \\
x_{j_{i}}{ }^{2}+y_{j_{i}}{ }^{2}{ }^{2} \\
x_{j_{i+1}}{ }^{2}+y_{j_{i+1}}
\end{array}\right\}
$$

where

$$
r_{j_{i}}^{2}=\gamma_{j_{i}}+\alpha_{j_{i}}^{2}+\beta_{j_{i}}^{2}
$$

Solving Eq. (39) and substituting $\alpha_{j_{i}}, \beta_{j_{i}}$ and $\gamma_{j_{i}}$ into Eq. (40) yields $r_{j_{i}}$. Further substitution into Eq. (37) determines the curvature $\kappa_{j_{i}}$ of the target profile at $\mathbf{z}_{j_{i}}$. Alternatively, the radius of curvature can be computed for each point on the $e^{t h}$ segment as

$$
r_{j_{i}}=\frac{\left\|\mathbf{z}_{j_{i+1}}-\mathbf{z}_{j_{i-1}}\right\|}{2 \sin \phi} i=k_{j}^{e}+1 \ldots, k_{j}^{e+1}-1 \quad j=1, \ldots, p
$$

where $\phi$ is the angle $\angle \mathbf{z}_{j_{i-1}} \mathbf{z}_{j_{i}} \mathbf{z}_{j_{i+1}}[100]$.

The sign of curvature $\kappa_{j_{i}}$ is designated positive if $\mathbf{z}_{j_{i-1}}, \mathbf{z}_{j_{i}}$ and $\mathbf{z}_{j_{i+1}}$ form a counterclockwise movement and is determined from direction vectors [101, 102]. A direction vector $\mathbf{P}_{j_{i-1}}$ is defined such that it extends from $\mathbf{z}_{j_{i-1}}$ to $\mathbf{z}_{j_{i}}$.

$$
\mathbf{P}_{j_{i-1}}=\left\{\begin{array}{c}
x_{j_{i}}-x_{j_{i-1}} \\
y_{j_{i}}-y_{j_{i-1}}
\end{array}\right\}
$$

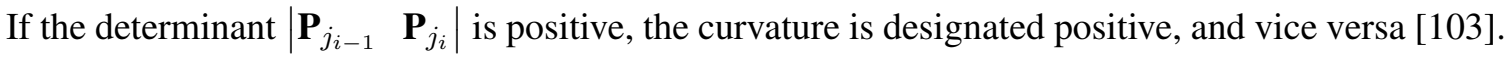

The first and last points of any profile have no curvature. In order to apply the curvature smoothing algorithm that is described in the next section, the first point is taken to have the curvature of the second point and the last point that of the second-to-last point. Thus, $\kappa_{j_{1}}=\kappa_{j_{2}}$ and $\kappa_{j_{n_{j}^{*}}}=\kappa_{j_{n_{j}^{*}-1}}$.

\subsubsection{Curvature Smoothing}

The curvature generated at all points along a profile establishes a curvature function. The curvature function may vary significantly between neighboring points along a target profile. Since 
large-scale matching of the profiles is desired, the curvature function can be smoothed to eliminate the irregularities. The smoothing of the curvatures is accomplished with a weighted moving average technique $[104,105]$. Excluding the first and last points, the smoothed curvature of the $j^{\text {th }}$ target profile at the $i^{t h}$ point is calculated as

$$
\tilde{\kappa}_{j_{i}}=\frac{1}{4} \kappa_{j_{i-1}}+\frac{1}{2} \kappa_{j_{i}}+\frac{1}{4} \kappa_{j_{i+1}}
$$

The smoothed curvatures of the first and last points are calculated as

$$
\begin{gathered}
\tilde{\kappa}_{j_{1}}=\frac{2}{3} \kappa_{j_{1}}+\frac{1}{3} \kappa_{j_{2}}, \\
\tilde{\kappa}_{j_{n_{j}^{*}}}=\frac{1}{3} \kappa_{j_{n_{j-1}^{*}}}+\frac{2}{3} \kappa_{j_{n_{j}^{*}}} .
\end{gathered}
$$

Successive passes of the curvature data through Eqs. (43), (44), and (45) will decrease the fluctuations in the curvature function. Unlike increasing the half-width of the moving average, this method preserves curvature data at the end of the profiles. Additionally, repeatedly applying the smoothing function allows the designer to visually assess the acceptability of the curvature information after each pass.

Two target profiles are shown as the solid curves in Fig. 29a. The curvature calculated from Eqs. (37), (39), and (40) is shown in Fig. 29b. The smoothed curvature calculated with five iterations through Eqs. (43), (44), and (45) is shown in Fig. 29c. Note that the smoothed curvature plot has less scatter and this may provide a better instrument to identify regions of similar curvature. However, the limitation is discussed in Section 2.3.3.

\subsubsection{Regenerating the Target Profile}

A new type of target profile, unused through the remainder of this dissertation but with potential benefits, may be generated using the smoothed curvature function and the average piece length $\bar{s}_{m}$. This ensures a constant spacing between points along the target profile and it visually confirms that 


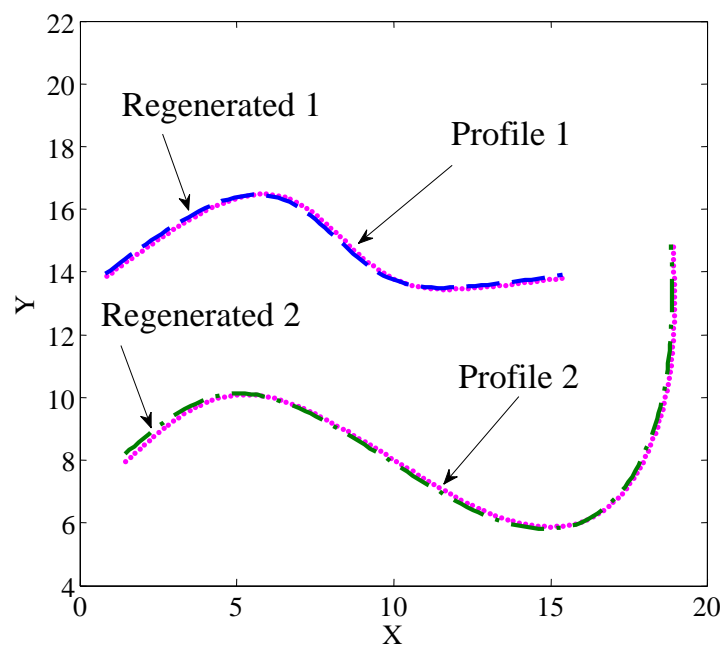

(a) Target Profiles

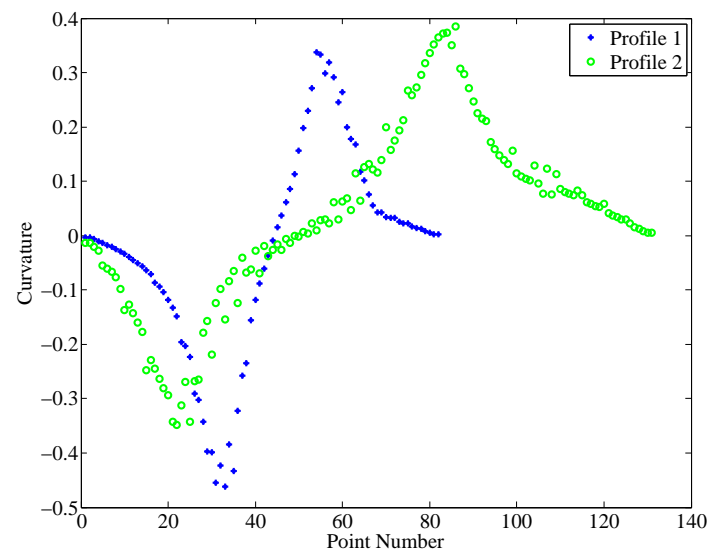

(b) Curvature Plot

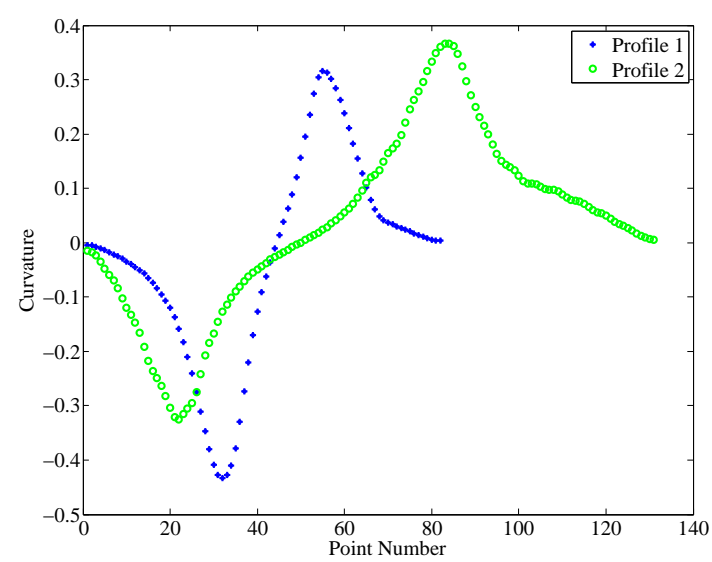

(c) Smoothed Curvature

Figure 29: Target profiles (a) with calculated curvature (b) and smoothed curvature (c). The dashed curves in (a) are regenerated profiles and are nearly identical to the original profiles. 
smoothing does not necessarily affect the accuracy of the target profiles in relation to the design profiles. To confirm this, an available tool that calculates the point-to-point errors between the original and the regenerated target profiles may be utilized. Specifically, the location of a regenerated point $\tilde{\mathbf{z}}_{j_{i+1}}$ is constructed from $\tilde{\mathbf{z}}_{j_{i-1}}, \tilde{\mathbf{z}}_{j_{i}}$ and $\tilde{r}_{j_{i}}$, where $\tilde{r}_{j_{i}}=1 / \tilde{\kappa}_{j_{i}}$.

The center of the arc, with radius $\tilde{r}_{j_{i}}$, passing through $\tilde{\mathbf{z}}_{j_{i-1}}$ and $\tilde{\mathbf{z}}_{j_{i}}$ is designated as $\left\{\tilde{\alpha}_{j_{i}}, \tilde{\beta}_{j_{i}}\right\}^{T}$ and is determined by using the equation of a circle,

$$
\tilde{\alpha}_{j_{i}}^{2}+\tilde{\beta}_{j_{i}}^{2}-2 \tilde{\alpha}_{j_{i}} x-2 \tilde{\beta}_{j_{i}} y=\tilde{r}_{j_{i}}^{2}-x^{2}-y^{2}
$$

Substituting the coordinates of $\tilde{\mathbf{z}}_{j_{i-1}}$ and $\tilde{\mathbf{z}}_{j_{i}}$ into Eq. (46) and expressing in matrix form yields

$$
\left[\begin{array}{cc}
-2 \tilde{x}_{j_{i-1}} & 1 \\
-2 \tilde{x}_{j_{i}} & 1
\end{array}\right]\left\{\begin{array}{c}
\tilde{\alpha}_{j_{i}} \\
\tilde{\alpha}_{j_{i}}^{2}+\tilde{\beta}_{j_{i}}^{2}
\end{array}\right\}-\tilde{\beta}_{j_{i}}\left\{\begin{array}{c}
2 \tilde{y}_{j_{i-1}} \\
2 \tilde{y}_{j_{i}}
\end{array}\right\}=\left\{\begin{array}{c}
\tilde{r}_{j_{i}}{ }^{2}-\tilde{x}_{j_{i-1}}^{2}-\tilde{y}_{j_{i-1}}^{2} \\
\tilde{r}_{j_{i}}{ }^{2}-\tilde{x}_{j_{i}}^{2}-\tilde{y}_{j_{i}}^{2}
\end{array}\right\}
$$

Being second order, two sets of $\left\{\tilde{\alpha_{j_{i}}}, \tilde{\beta_{j_{i}}}\right\}^{T}$ satisfy Eq. (47). The sign of $\tilde{r}_{j_{i}}$ is used to identify the correct center point.

Since $\tilde{\mathbf{z}}_{j_{i+1}}$ also shares the center point, substituting into Eq. (46) gives

$$
\tilde{\alpha}_{j_{i}}^{2}+\tilde{\beta}_{j_{i}}^{2}-2 \tilde{\alpha}_{j_{i}} \tilde{x}_{j_{i+1}}-2 \tilde{\beta}_{j_{i}} \tilde{y}_{j_{i+1}}=\tilde{r}_{j_{i}}^{2}-\tilde{x}_{j_{i+1}}^{2}-\tilde{y}_{j_{i+1}}^{2}
$$

The regenerated target profiles are constructed with constant linear piece length $\bar{s}_{m}$. Thus, $\tilde{\mathbf{z}}_{j_{i+1}}$ will be spaced a distance $\bar{s}_{m}$ from $\tilde{\mathbf{z}}_{j_{i}}$, giving

$$
\left(\tilde{x}_{j_{i+1}}-\tilde{x}_{j_{i}}\right)^{2}+\left(\tilde{y}_{j_{i+1}}-\tilde{y}_{j_{i}}\right)^{2}=\bar{s}_{m}^{2}
$$

Equations (48) and (49) can be reduced into a second order equation that will produce two sets of $\left\{\tilde{x}_{j_{i+1}}, \tilde{y}_{j_{i+1}}\right\}^{T}$. One solution will be identical to $\left\{\tilde{x}_{j_{i-1}}, \tilde{y}_{j_{i-1}}\right\}^{T}$, which is discounted, leaving one acceptable solution. Figure 30 illustrates the graphical solution to Eqs. (48) and (49). Thus, the location of $\tilde{\mathbf{z}}_{j_{i+1}}$ is regenerated from $\tilde{\mathbf{z}}_{j_{i-1}}, \tilde{\mathbf{z}}_{j_{i}}$ and $\tilde{\kappa}_{j_{i}}$.

Starting with points 1 and 2, where $\tilde{\mathbf{z}}_{j_{1}}=\mathbf{z}_{j_{1}}$ and $\tilde{\mathbf{z}}_{j_{2}}=\mathbf{z}_{j_{2}}$, the subsequent regenerated points on each target profile can be successively constructed. By superimposing a plot of the regenerated 


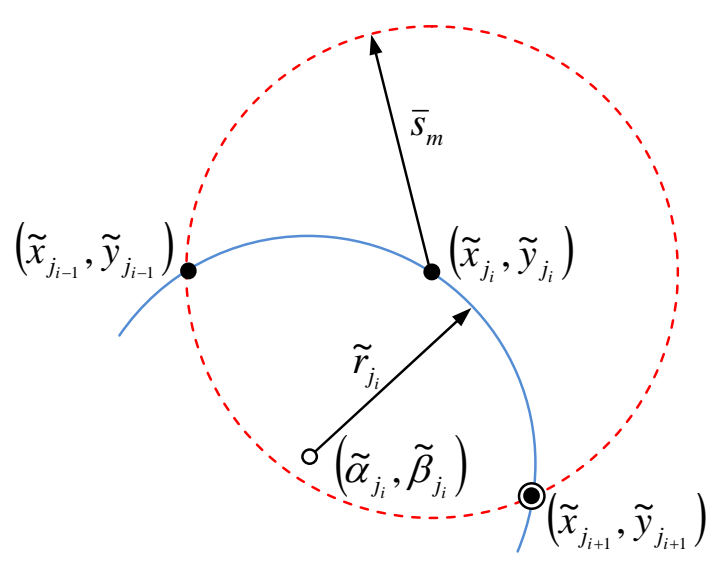

Figure 30: The location of $\tilde{\mathbf{z}}_{j_{i+1}}$ is reconstructed from $\tilde{\mathbf{z}}_{j_{i-1}}, \tilde{\mathbf{z}}_{j_{i}},\left(\tilde{\alpha}_{j_{i}}, \tilde{\beta}_{j_{i}}\right)$ and $\tilde{r}_{j_{i}}$.

profiles after each smoothing pass, the designer is able to confirm that the curvature smoothing does not appreciably alter the intended shape change. The initial target profiles are shown as the solid curves in Fig. 29a, whereas the dashed curves represent the regenerated profiles using the smoothed curvature. Note, however, that while the original target profiles still represent the design profiles, these regenerated target profiles have been modified. Hence, the suitability of the regenerated profiles depends on whether they represent the design profiles in a sufficient manner, based on the application.

The advantage of a regenerated target profile is that the same number of contiguous pieces along all of the target profiles has exactly the same piece length. Moreover, arc lengths for segments containing different numbers of pieces are related only by the number of pieces included in the segments, which is not true of the target profiles proposed earlier in this chapter. The disadvantage that comes with this new type of profile is that it gives rise to an additional (though potentially modest) loss in fidelity versus the design profiles as exhibited in Fig. 29a. 
The implemented approach incorporates some of the techniques in the regenerated target profile approach. Calculating curvature of each point of the target profiles may assist the algorithm detecting where $\mathscr{C}$-segments are best placed. Consequently, this would eliminate the need for multiple initial guesses for the segment matrix $S M$ as explained in Sec. 3.1.1 and would accelerate the overall design process. 


\section{CHAPTER III}

\section{THE SEGMENTATION PROCESS}

This chapter presents the process to create a chain of rigid-body segments from a set of open, closed, or fixed-end profiles. After a set of target profiles has been generated, each curve is divided into sections that correspond to segments that will be created to form the chain of rigid-bodies. A design vector specifies the segment types in the chain. The vector dictates the creation of an initial segment matrix. Based on the matrix, $\mathscr{M}$ - and $\mathscr{C}$-segments are created and segment distance errors calculated. The unconnected segments can be improved depending on their errors. The process can be repeated with other design vectors and tried with many starting guesses. A resulting set of segments can be connected and its location optimized versus the set of target profiles.

\subsection{A New Segmentation Process for Target Profiles of Different Arc Lengths}

Certain conditions exist for identifying the $q$ groups of points that will form segments on each

of the target profiles. The number of pieces on the $e^{t h}$ segment of the $j^{\text {th }}$ profile is designated $m_{j}^{e}$, and the number of points is $n_{j}^{e}=m_{j}^{e}+1$. Since an $\mathscr{M}$-segment represents a single rigid link, a fundamental constraint on forming $\mathscr{M}$-segments is that the number of pieces in those segments should be constant across all $p$ profiles. That is, if the $e^{t h}$ segment is an $\mathscr{M}$-segment, $m_{1}^{e}=m_{2}^{e}=$ $\ldots=m_{p}^{e}$. Since the $\mathscr{C}$-segments are used to compensate for differences in length among the 
profiles, the number of pieces in corresponding $\mathscr{C}$-segments from each profile will generally differ. The number of pieces in the $\mathscr{C}$-segments on the $j^{\text {th }}$ profile must be selected such that $\sum_{e=1}^{q} m_{j}^{e}=m_{j}^{*}$.

The points that lie on the boundary between segments are termed segmentation points, which also include the first and last points on the target profile. Points on the $j^{\text {th }}$ target profile are numbered 1 through $n_{j}^{*}$. The index of the segmentation point at the start of the $e^{t h}$ segment on the $j^{\text {th }}$ profile is designated as $k_{j}^{e}$. The index of the final point of that segment is $k_{j}^{e+1}$. Note that $k_{j}^{e+1}$ is also the index of the first point on the $e+1$ segment. Thus,

$$
\begin{aligned}
& k_{j}^{1}=1 \quad j=1, \ldots, p \\
& k_{j}^{e}=k_{j}^{e-1}+m_{j}^{e-1}=1+\sum_{i=1}^{e-1} m_{j}^{i} \quad e=2, \ldots, q \quad j=1, \ldots, p \\
& k_{j}^{q+1}=n_{j}^{*} \quad j=1, \ldots, p .
\end{aligned}
$$

The same number of segments $q$ and their types (i.e., $\mathscr{M}$ - or $\mathscr{C}$-segments) must be maintained for all profiles. A design vector $\mathbf{V}$ defines the number of segments and their types in a candidate design. As an example, $\mathbf{V}=[\mathscr{M} \mathscr{C} \mathscr{M} \mathscr{C}]$ specifies that four segments $(q=4)$ will be formed, with the first being an $\mathscr{M}$-segment, followed by a $\mathscr{C}$-segment, an $\mathscr{M}$-segment and a $\mathscr{C}$-segment. Currently, an a priori method to determine the error minimizing $\mathbf{V}$ for a set of design profiles is lacking. The designer can specify $\mathbf{V}$ after inspecting the shape of the profiles and by comparing their curvature plots like those generated in Figs. 29a and 29b. Any straight portions of different lengths or similarly curved portions of different lengths are candidates for $\mathscr{C}$-segments. Alternatively, the error minimizing process can cycle through numerous design vectors to attain an adequate fit to the set of target profiles. This may have a long computation time depending on the number of segments and the computer processing capability. Any combination of $\mathscr{M}$ - or $\mathscr{C}$-segments is possible. For a design with $q$ segments, there are $2^{q}-1$ different design vectors that contain at least one $\mathscr{C}$-segment. 
It is noted that a $\mathscr{C}$-segment represents two rigid links, while an $\mathscr{M}$-segment represents one rigid link in the shape-approximating chain.

\subsubsection{The Segment Matrix}

A $p \times q$ segment matrix $S M$ identifies the number of pieces in each segment and is constructed consistent with the design vector. The $j^{\text {th }}$ row of $S M$ represents the $j^{\text {th }}$ target profile and contains the number of pieces for each segment on that profile. From an implementation standpoint, segments should not be so small that a physical embodiment is impractical. A minimum number of segment pieces $\alpha$ is defined such that $m_{j}^{e} \geq \alpha, e=1, \ldots, q, j=1, \ldots, p$.

As an example, consider the set of three target profiles from Fig. 25 having $m_{1}^{*}=76, m_{2}^{*}=90$, and $m_{3}^{*}=99$ pieces, respectively. In order not to have any short segments, $\alpha=5$ was specified. With $\mathbf{V}=[\mathscr{M} \mathscr{C} \mathscr{M} \mathscr{C}]$, an initial segment matrix may be constructed as

$$
S M_{1}=\left[\begin{array}{cccc}
m_{1}^{1} & m_{1}^{2} & m_{1}^{3} & m_{1}^{4} \\
m_{2}^{1} & m_{2}^{2} & m_{2}^{3} & m_{2}^{4} \\
m_{3}^{1} & m_{3}^{2} & m_{3}^{3} & m_{3}^{4}
\end{array}\right]=\left[\begin{array}{cccc}
15 & 13 & 9 & 38 \\
15 & 9 & 9 & 56 \\
15 & 39 & 9 & 35
\end{array}\right] .
$$

Since the first segment is an $\mathscr{M}$-segment, the same number of pieces has been selected to be used on all profiles: $m_{j}^{1}=15, j=1, \ldots, 3$. Similarly for the third segment, $m_{j}^{3}=9, j=1, \ldots, 3$. Being $\mathscr{C}$-segments, all instances of the second and fourth segments do not need to have the same number of pieces. The initial numbers of pieces in each instance of the second segment $\left(m_{j}^{2}\right)$ is individually specified. Being the final $\mathscr{C}$-segment in $\mathbf{V}$, the fourth segment $\left(m_{j}^{4}\right)$ will contain all remaining pieces in the profile. That is, $m_{1}^{4}=38$, such that for $j=1,15+13+9+38=76=m_{1}^{*}$, and likewise for the other profiles. The segmentation points can be calculated with Eqs. (50), (51), and (52). For $j=1, k_{1}^{1}=1, k_{1}^{2}=16, k_{1}^{3}=29, k_{1}^{4}=38$, and $k_{1}^{5}=76$. The segmentation points are indicated with black circles in Fig. 25.

Once an initial $S M$ is formulated, an iterative process creates the segments, evaluates how well they match the target profiles and adjusts $S M$ to minimize the error. The method for creating 
the segments is described in the following two sub-sections. The details of the evaluation and adjustment processes are explained in Section 3.2.

\subsection{2 $\mathscr{M}$-Segments}

The process of creating an $\mathscr{M}$-segment is identical to that described in Murray et al. [46] and is summarized here for completeness. The process begins by shifting all points on the segment to a common location so a single profile can be generated that approximates the shapes of all segments within the set. The first profile is selected as the common location, so each segment from the second through last profile is shifted to the first profile. Each point on the $e^{\text {th }}$ segment is shifted by a rigid body transformation in the plane as described in Section 2.2. Then, a new piecewise linear curve is constructed in which each point is the geometric center of the set of $p$ corresponding points in the shifted segments.

$$
\mathbf{Z}_{m_{i}}=\frac{1}{p}\left(\mathbf{z}_{1_{i}}+\sum_{j=2}^{p} \mathbf{Z}_{j_{i}}\right) \quad i=k_{j}^{e}, \ldots, k_{j}^{e+1} .
$$

This new curve becomes the $\mathscr{M}$-segment and represents one rigid RR link in the shape-changing chain. A second minimization process determines a rotation matrix $\hat{\mathbf{A}}_{j}^{e}$ and translation $\hat{\mathbf{d}}_{j}^{e}$ to shift instances of the mean segment back to profiles 2 through $p$ to approximate the shape of the segment on each target profile.

$$
\overline{\mathbf{z}}_{j_{i}}^{e}=\left(\hat{\mathbf{A}}_{j}^{e}\right)^{-1}\left(\mathbf{Z}_{m_{i}}-\hat{\mathbf{d}}_{j}^{e}\right) \quad i=k_{j}^{e}, \ldots, k_{j}^{e+1} \quad j=2, \ldots, p
$$

The first segments from the three profiles $(e=1, j=1,2,3)$ presented in Fig. 25 are shown in Fig. 31a and shifted to distance-minimizing positions relative to the first profile in Fig. $31 \mathrm{~b}$. Figure 31c shows the creation of an $\mathscr{M}$-segment that approximates the set, which is shifted back to each target profile in Fig. 31d. 


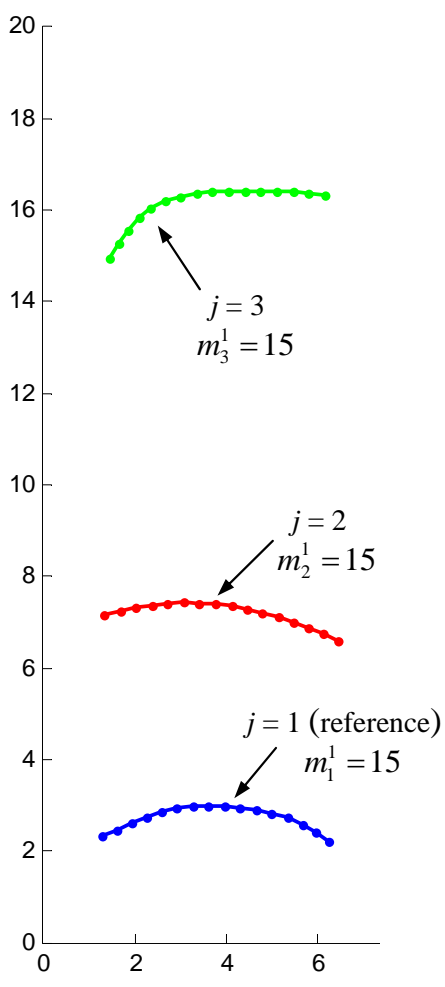

(a)

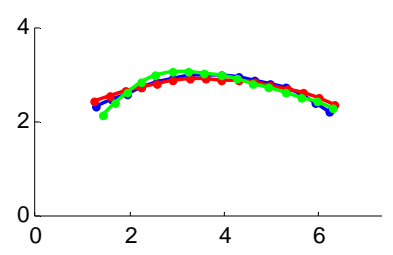

(b)

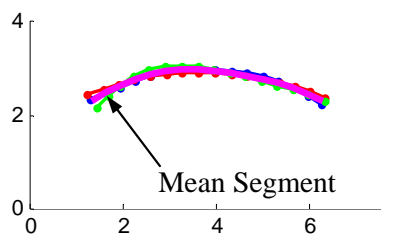

(c)

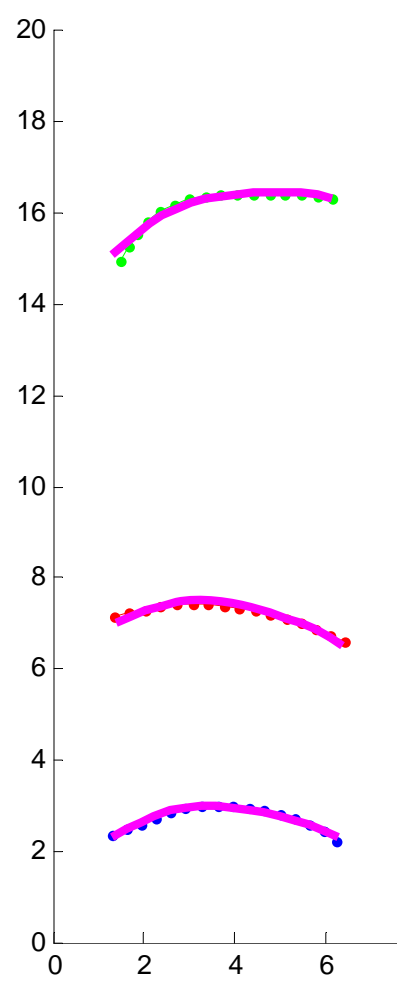

(d)

Figure 31: (a) The first segment from three target profiles to be represented by one mean segment. (b) Segments 2 and 3 shifted to reference segment 1 in a distance minimizing transformation. (c) The mean segment is generated as the average of the corresponding segment points. (d) The mean segment is shifted back to the original segments in another distance minimizing transformation.

\subsection{3 $\mathscr{C}$-Segments}

A constant curvature $\mathscr{C}$-segment is created using discrete, signed, radius-of-curvature values at each point along the segment for all target profiles [104]. The radius of curvature value of the $j^{\text {th }}$ target profile at the $i^{t h}$ point is the radius of an $\operatorname{arc} r_{j_{i}}$ that passes through $\mathbf{z}_{j_{i}}$ and the neighboring points $\mathbf{z}_{j_{i-1}}$ and $\mathbf{z}_{j_{i+1}}$ [99]. Discussion of the radius of curvature is found in Section 2.3.1. The 
mean radius of the $e^{t h}$ segment on all profiles is

$$
\bar{r}^{e}=\frac{1}{\sum_{j=1}^{p} m_{j}^{e}-1}\left(\sum_{j=1}^{p}\left(\sum_{i=k_{j}^{e}+1}^{k_{j}^{e+1}-1} r_{j_{i}}\right)\right),\left(\bar{r}^{e}, 0\right)(0,0) .
$$

The curvatures of the first and last points are not included in the average.

Instances of a $\mathscr{C}$-segment are created by generating points $\overline{\mathbf{Z}}_{j_{i}}^{e}$ along a radius $\bar{r}^{e}$ and arc length

$$
L_{j}^{e}=m_{j}^{e} \bar{s}^{e}
$$

where the average length of the pieces on the $e^{t h}$ segment on all $p$ profiles is

$$
\bar{s}^{e}=\sum_{j=1}^{p}\left(\frac{1}{m_{j}^{e}}\left(\sum_{i=k_{j}^{e}+1}^{k_{j}^{e+1}-1} s_{j_{i}}\right)\right)
$$

As with the $\mathscr{M}$-segments, instances of the $\mathscr{C}$-segment are placed at each target profile in a distance minimizing transformation using Eq. (55). The construction of the segment is shown in Fig. 32.

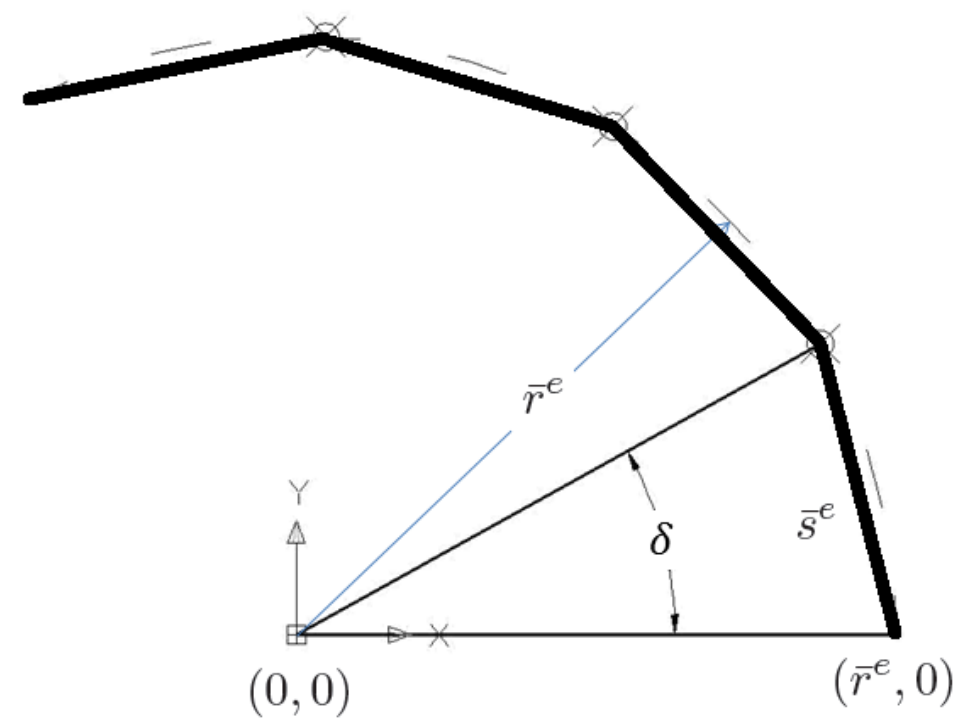

Figure 32: A $\mathscr{C}$-segment is built and then transformed to its location in a distance minimizing configuration. 
Each of the second segments from the three profiles $(e=2, j=1,2,3)$ presented in Fig. 25 are shown in Fig. 33a with a mean piece length of $\bar{s}_{m}=0.35$ and each having a different number of pieces $\left(m_{1}^{1}=13, m_{2}^{1}=9\right.$ and $\left.m_{3}^{1}=39\right)$. A mean radius is calculated as $\bar{r}^{1}=15.06$, and arcs with lengths of $L_{1}^{1}=4.55, L_{2}^{1}=3.42$ and $L_{3}^{1}=13.65$ are constructed and fit through the profiles as shown in Fig. 33b.

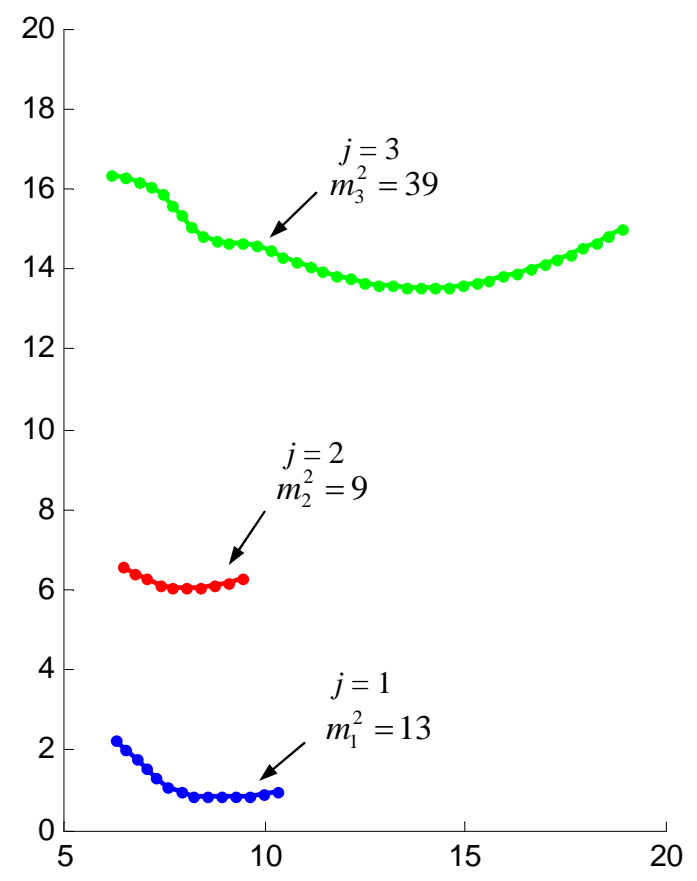

(a)

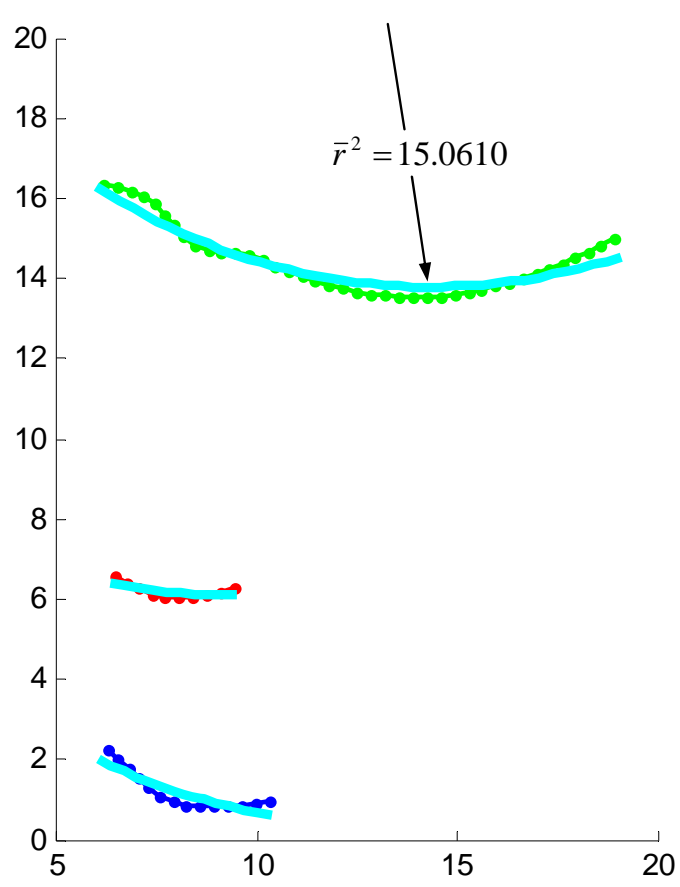

(b)

Figure 33: (a) The second segment from three target profiles approximated with a $\mathscr{C}$-segment. (b) An arc of the same radius, but varying length, approximates the segment on all profiles.

\subsection{Evaluating and Adjusting the Segment Matrix}

Once instances of the segments are created as specified by $S M$, their shape-approximation errors are evaluated. The maximum point-to-point distance on the $j^{\text {th }}$ instance of the $e^{\text {th }}$ segment 
to the corresponding point on the $j^{\text {th }}$ profile is

$$
E_{j}^{e}=\max \left|\overline{\mathbf{z}}_{j_{i}}^{e}-\mathbf{z}_{j_{i}}\right| \quad i=k_{j}^{e}, \ldots, k_{j}^{e+1}
$$

Similar to $S M$, a $p \times q$ error matrix $E M$ organizes $E_{j}^{e}$ for all segments on all profiles. Error metrics that assist in segment adjustment include the maximum overall error,

$$
E_{\max }=\max \left(E_{j}^{e}\right) \quad e=1, \ldots, q \quad j=1, \ldots, p,
$$

the mean error of the $e^{\text {th }}$ segment,

$$
\bar{E}^{e}=\sum_{j=1}^{p} E_{j}^{e}
$$

and the overall mean error

$$
\bar{E}=\frac{1}{q} \sum_{e=1}^{q}\left(\frac{1}{p} \sum_{j=1}^{p} E_{j}^{e}\right)
$$

An example of the EM that corresponds with the profiles from Fig. 25, and the initial $S M$ of Eq. (53) is

$$
E M_{1}=\left[\begin{array}{cccc}
E_{1}^{1} & E_{1}^{2} & E_{1}^{3} & E_{1}^{4} \\
E_{2}^{1} & E_{2}^{2} & E_{2}^{3} & E_{2}^{4} \\
E_{3}^{1} & E_{3}^{2} & E_{3}^{3} & E_{3}^{4}
\end{array}\right]=\left[\begin{array}{cccc}
0.10 & 0.29 & 0.04 & 0.43 \\
0.15 & 0.18 & 0.03 & 1.37 \\
0.19 & 0.24 & 0.06 & 1.50
\end{array}\right] .
$$

In Eq. (63), $E_{\max }=1.50$ and $\bar{E}=0.38$. For the $\mathscr{M}$-segments, $\bar{E}^{1}=0.15$ and $\bar{E}^{3}=0.04$.

The number of pieces in each segment can be changed to improve the shape approximation and consequently reduce $E_{\max }$. Adjustments to $S M$ involve adding, removing or preserving the number of pieces in each segment in order to balance the errors, i.e., $E_{\max }$ approaches $\bar{E}$. For a $\mathscr{C}$ segment, each $E_{j}^{e}$ is compared to $\bar{E}$. If $E_{j}^{e}<\bar{E}$, the segment fits the profile better than the average and can be lengthened to balance the error. A change $\delta m_{j}^{e}=+1$ is assigned to that instance of the segment, meaning that one piece will be added. Conversely, if $E_{j}^{e}>\bar{E}$, that instance of the segment has a below average fit and $\delta m_{j}^{e}=-1$ is assigned, meaning that one piece will be removed.

Because all $\mathscr{M}$-segments must be adjusted the same amount, each $\bar{E}^{e}$ is compared to $\bar{E}$. If $\bar{E}^{e}<$ $\bar{E}$, a change is assigned to all profiles $\delta m_{j}^{e}=+1, j=1, \ldots, p$. Since a segment cannot violate the 
minimum number of pieces constraint, a $\delta m_{j}^{e}=0$ may be assigned if $m_{j}^{e}=\alpha$. Additionally, the total number of pieces on each profile must remain the same,

$$
\sum_{e=1}^{q} \delta m_{j}^{e}=0, \quad j=1, \ldots, p .
$$

As in the generation of $S M$, the final $\mathscr{C}$-segment within $\mathbf{V}$ is not adjusted according to its error. Instead, it is adjusted to ensure that the $j^{\text {th }}$ profile retains $m_{j}^{*}$ pieces. Hence, either no change or changes greater than one may be assigned to the instances of the last $\mathscr{C}$-segment. Nevertheless, more sophistication can be incorporated whereby the remaining pieces are taken by another $\mathscr{C}$-segment in the chain.

The changes are added to $S M$ to obtain an adjusted $S M$ with which a new segmentation trial is conducted. The iterative process continues, assessing errors at each step in order to achieve a final segment matrix with the lowest point-to-point error. The iterations are stopped when the current value of $E_{\max }$ is greater than or equal to the previous five values. To avoid a local minima, these error-reducing iterations are performed on several initial $S M$ matrices. The $S M$ associated with the lowest value of $E_{\max }$ within the entire error history is deemed as the distance minimizing set of segments. These error-reducing iterations were conducted on the four-segment chain of Fig. 25 and the $S M$ of Eq. (53). Many local minima may be found using this process. For problems with a larger number of pieces, the number of initial segment matrices allowed may be increased accordingly before the iteration is terminated. Consequently, in this example, the segment matrix with the lowest $E_{\max }$ is

$$
S M_{f}=\left[\begin{array}{llll}
19 & 17 & 20 & 19 \\
19 & 16 & 20 & 34 \\
19 & 36 & 20 & 23
\end{array}\right]
$$

which resulted in an error matrix

$$
E M_{f}=\left[\begin{array}{llll}
0.17 & 0.23 & 0.22 & 0.11 \\
0.26 & 0.03 & 0.17 & 0.22 \\
0.19 & 0.38 & 0.11 & 0.31
\end{array}\right]
$$


The error metrics were reduced to $E_{\max }=0.38$ and $\bar{E}=0.20$ (from $E_{\max }=1.50$ and $\bar{E}=0.38$ with $S M_{1}$ ). The rigid-body chain associated with the initial segmentation is shown in Fig. 34a, whereas Fig. 34b shows the chain after the error-reducing iterations.

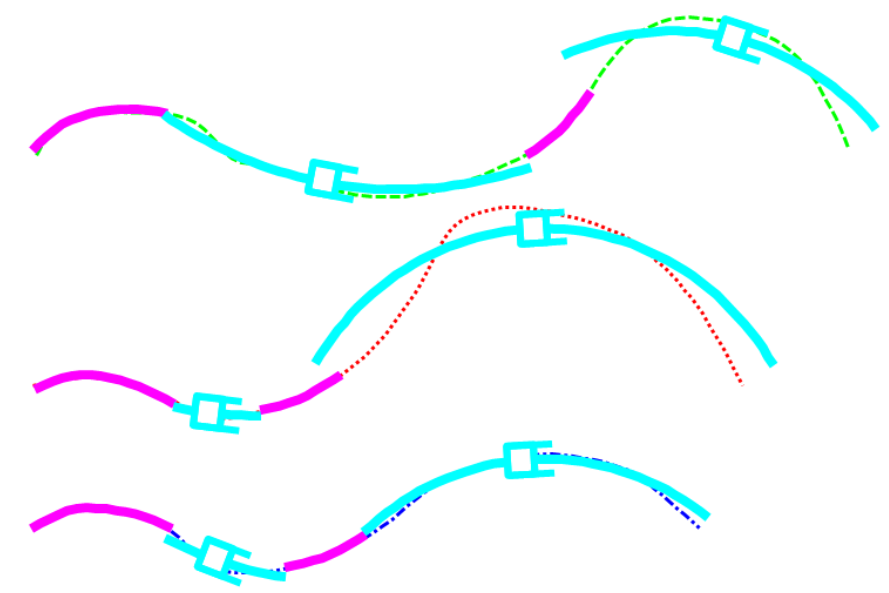

(a)

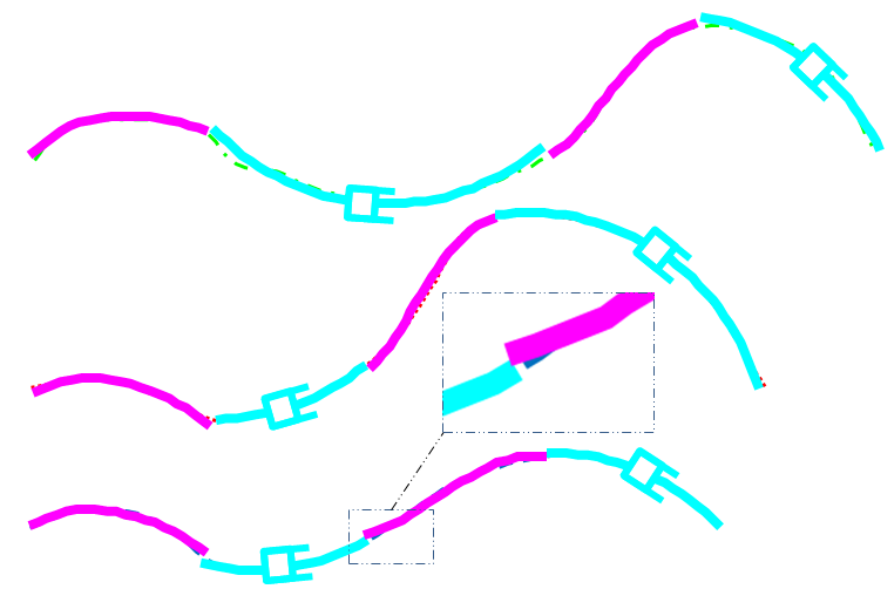

(b)

Figure 34: A rigid-body chain that approximates the target profiles. The chain after the initial segmentation matrix is shown in (a) with $E_{\max }=1.50$. The chain after the error-reducing iterations is shown (b) with $E_{\max }=0.38$. The inset illustrates that segmentation points are not coincident. 


\subsection{Joining the Chain}

Since the segments are generated individually, the segmentation point on one segment will not coincide with the corresponding segmentation point on the adjoining segment. As shown in Fig. 34b, $\overline{\mathbf{z}}_{j_{i}}^{e} \neq \overline{\mathbf{z}}_{j_{i}}^{e+1}, \forall i=k_{j}^{e}, \quad e=2, \ldots, q$ and $j=1, \ldots, p$. Since the segments will be connected at their endpoints with revolute joints, each segment must be slightly moved from its error-minimizing position to unite the segmentation points. During this process, each profile is treated individually.

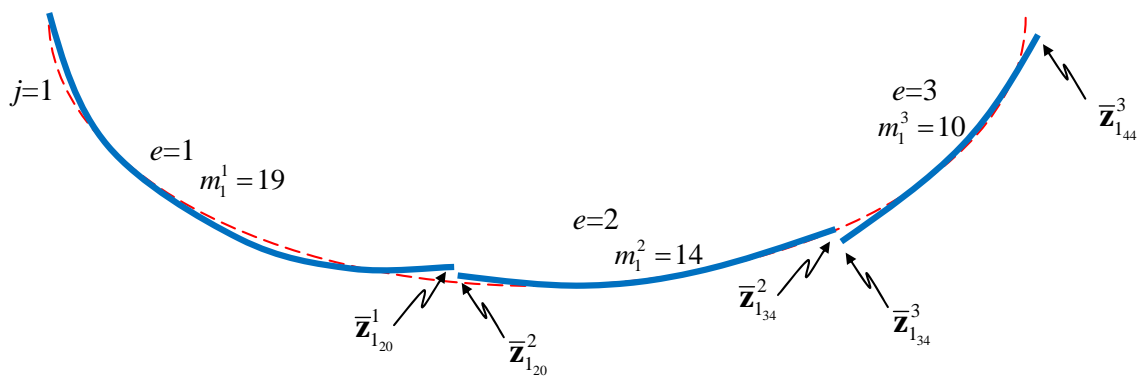

(a) Segmentation points do not coincide

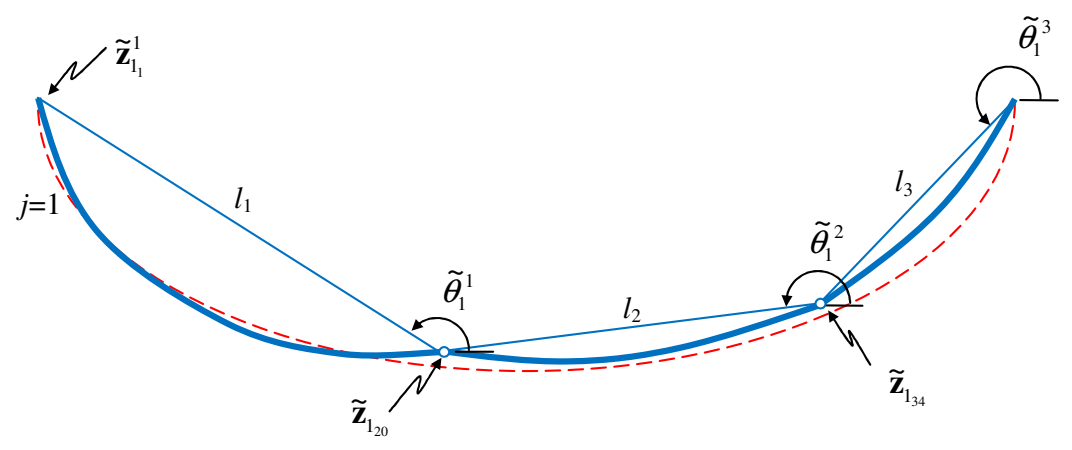

(b) Segments are repositioned to unite segmentation points

Figure 35: After instances of segments are created, the segmentation points will not coincide. To merge the segmentation points and form a chain connected by revolute joints, each segment must be repositioned by adjusting $\tilde{\mathbf{z}}_{j_{1}}^{1}$ and $\tilde{\theta}_{e}$. 
Each point on each segment of the $j^{\text {th }}$ profile is relocated by

$$
\tilde{\mathbf{z}}_{j_{i}}^{e}=\tilde{\mathbf{A}}_{j}^{e} \overline{\mathbf{z}}_{j_{i}}^{e}+\tilde{\mathbf{d}}_{j}^{e}, \quad i=1, \ldots, n_{j}^{*}, \quad e=1, \ldots, m_{j}
$$

where

$$
\tilde{\mathbf{A}}_{j}^{e}=\left[\begin{array}{cc}
\cos \tilde{\theta}_{j}^{e} & -\sin \tilde{\theta}_{j}^{e} \\
\sin \tilde{\theta}_{j}^{e} & \cos \tilde{\theta}_{j}^{e}
\end{array}\right], \tilde{\mathbf{d}}_{j}^{e}=\left[\begin{array}{c}
\tilde{x}_{j}^{e} \\
\tilde{y}_{j}^{e}
\end{array}\right] .
$$

The endpoints of adjacent segments must be coincident, which formulates the principal constraint,

$$
\tilde{\mathbf{z}}_{j_{i}}^{e}=\tilde{\mathbf{z}}_{j_{i}}^{e+1}=\tilde{\mathbf{z}}_{j_{i}} \forall i=k_{j}^{e}, \quad e=2, \ldots, q, \quad j=1, \ldots, p
$$

For closed-loop target profiles, additional constraints include

$$
\sum_{e=1}^{q} l_{e} \cos \tilde{\theta}_{j}^{e}=0, \quad \sum_{e=1}^{q} l_{e} \sin \tilde{\theta}_{j}^{e}=0, \quad j=1, \ldots, p,
$$

where $l_{e}$ is the length of the line segment connecting the first and last point on segment $e$. For fixed-end target profiles, additional constraints include

$$
\tilde{\mathbf{z}}_{j_{1}}^{1}=\mathbf{z}_{j_{1}}, \quad \tilde{\mathbf{z}}_{j_{n_{j}^{*}}^{q}}^{q}=\mathbf{z}_{j_{n_{j}^{*}}}
$$

As in Persinger et al. [79], the segmentation points are united through a numerical optimization to determine $\tilde{\mathbf{z}}_{j_{1}}^{e} e=2, \ldots, q$ subject to Eq. (68), and if appropriate, Eq. (69) or (70). As shown in Fig. 35b, the location of the first point on each $j^{\text {th }}$ instance of the chain $\tilde{\mathbf{z}}_{j_{1}}^{1}$, the angle of each segment instance $\tilde{\theta}_{j}^{e}$, and the translation $\tilde{\mathbf{d}}_{j}^{e}$ are the $q+2$ optimization variables. The objective function $f$ to be minimized is the sum of the squared point-to-point distances between points on the instance of the segments and the points on the $j^{\text {th }}$ target profile,

$$
f=\sum_{i=1}^{k_{j}^{e}}\left[\tilde{\mathbf{z}}_{j_{i}}^{e}-\mathbf{z}_{j_{i}}\right]^{T}\left[\tilde{\mathbf{z}}_{j_{i}}^{e}-\mathbf{z}_{j_{i}}\right] \quad e=1, \ldots, q .
$$

The fmincon function in the optimization toolbox of MATLAB is well suited for this type of constrained nonlinear multi-variable problem. The position of the segments as determined by the segmentation process generally serves as a suitable initial guess. 
Once optimized, $\tilde{\mathbf{z}}_{j_{i}}^{e}, e=2, \ldots, q, i=2, \ldots, n_{j}^{*}$ defining the chain of rigid links joined with revolute and prismatic joints is complete. The optimization process is conducted for the other profiles as well, resulting in a rigid-body chain that can be repositioned to approximate all target profiles.

The result of the joining process for the three profiles presented in Fig. 25 is shown in Fig. 36. After the error-reducing iterations on $S M$, the maximum point-to-point error was $E_{\max }=0.38$. However, after shifting segments to merge segmentation points, the maximum point-to-point error adjusted to $E_{\max }=0.39$ as shown in the error matrix

$$
E M_{\text {joined }}=\left[\begin{array}{llll}
0.26 & 0.15 & 0.25 & 0.11 \\
0.38 & 0.06 & 0.27 & 0.19 \\
0.26 & 0.33 & 0.39 & 0.36
\end{array}\right] .
$$

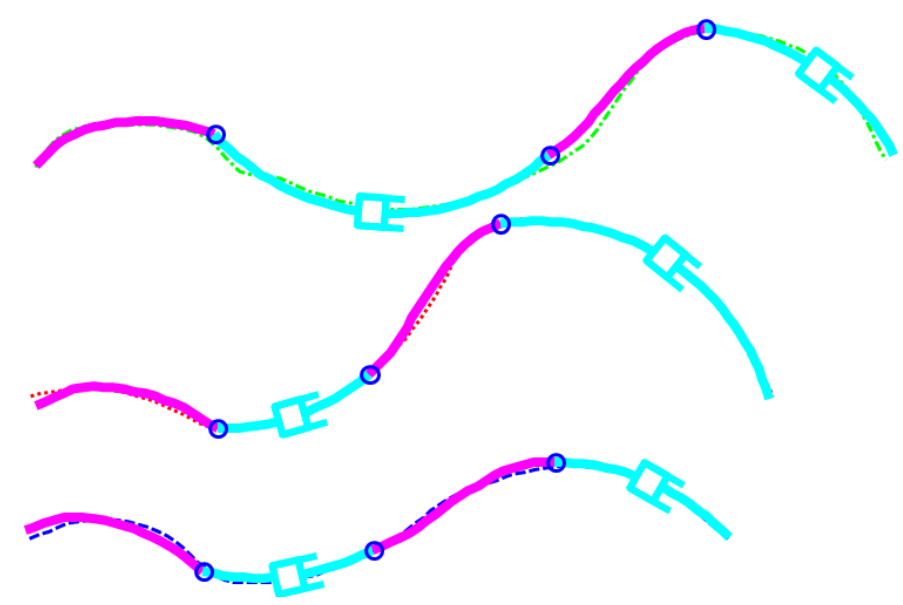

Figure 36: The joining process unites segmentation points, yet changes the error from $E_{\max }=0.38$ to $E_{\max }=0.39$.

If the accuracy of the approximation is deemed unsatisfactory, the designer can return to the segmentation phase and dictate a different design vector $\mathbf{V}$ or increase the number of segments. To compare alternative designs, the number of joints in the chain of rigid-bodies should be considered. 
$\mathscr{M}$-segments represent R-R links, so they contain two joints, whereas $\mathscr{C}$-segments represent R-P$\mathrm{R}$ chains and contain 3 joints. Generally, a chain of rigid-bodies with a greater number of joints provides a better approximation to the profiles at the expense of greater mechanical complexity. From the practical perspective of constructing a mechanism, it is desirable to find a compromise between the number of joints and the error between the chain of rigid-bodies and the target profiles.

\subsection{Compound Segment Types}

Since the number of links in the chain increases the mechanical complexity, it is practical to fuse some of the links together when the rotation of a revolute joint is small. To investigate the benefit of each revolute joint in the chain of rigid-bodies, the range of motion is calculated to determine whether the joint is necessary. Recall that $\mathbf{P}_{j_{i}}$ is the direction vector that extends from $\mathbf{z}_{j_{i-1}}$ to $\mathbf{z}_{j_{i}}$. Accordingly, the direction vector that extends from the preceding point to the $e^{t h}$ segmentation

point on the $j^{t h}$ profile is $\mathbf{P}_{j_{k_{j}^{e}}}$, and $\mathbf{P}_{j_{k_{j}+1}}$ is the vector from the $e^{t h}$ segmentation point to the next point. The relative joint angle at the $e^{t h}$ segmentation point is designated as $\sigma_{j}^{e}$ and is represented by the angle from $\mathbf{P}_{j_{k_{j}^{e}}}$ to $\mathbf{P}_{j_{k_{j}^{e}+1}}$. Thus, the range of motion exhibited by that joint is

$$
\Delta \sigma^{e}=\max \left(\sigma_{j}^{e}\right)-\min \left(\sigma_{l}^{e}\right) \quad j=1, \ldots, p, \quad l=1, \ldots, p .
$$

If the range of motion is smaller than some limit, $\Delta \sigma^{e}<\sigma_{\max }$, the revolute joint motion is considered insignificant during shape approximation. That joint may be considered unnecessary and eliminated to reduce the complexity. There are other metrics for selecting the value of $\sigma_{\max }$ dictating when to fuse segments such as a weighted average. The user defines an acceptable value for $\sigma_{\max }$ based on the sensitivity of the design to these joint rotations. If the revolute joint selected for fusing connects two $\mathscr{M}$-segments, the designer is advised to select a new design vector $\mathbf{V}$ with fewer segments. Experience has shown that creating a new $\mathscr{M}$-segment gives a lower segment error than that of two fused $\mathscr{M}$-segments. If the revolute joint connects a $\mathscr{C}$-segment with either 
another $\mathscr{C}$-segment or an $\mathscr{M}$-segment, the joint can be eliminated, fusing the segments at the mean angle. Note that it is possible to calculate the angle in other ways. Eliminating the joint creates a compound segment that fuses the two original segments. Returning to the example profiles of Fig. 25, the reduced-error chain of Fig. 34b is shown in Fig. 37a. However, the range of motion of the second revolute joint is $\Delta \sigma^{e}=12.18^{\circ}$ and may be considered insignificant. The segments were fused to create the chain shown in Fig. 37b. Eliminating the revolute joint increased the maximum point-to-point error from $E_{\max }=0.39$ to $E_{\max }=0.45$. However, the number of segments and kinematic complexity have been reduced at a modest expense of error.

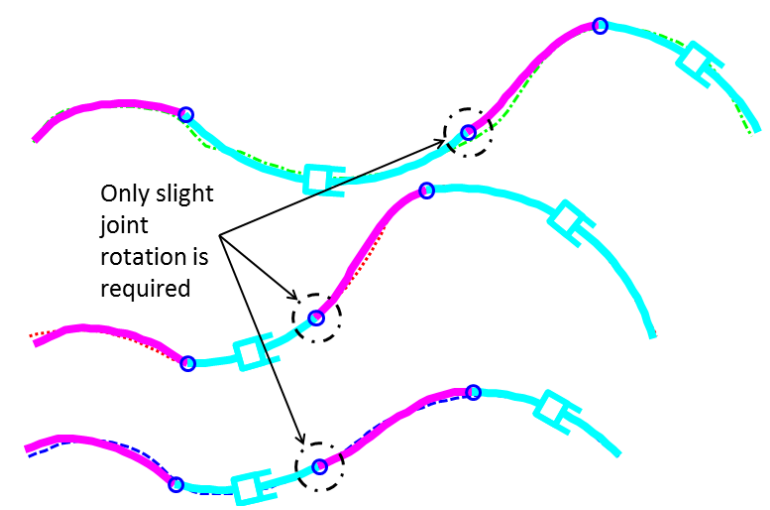

(a)

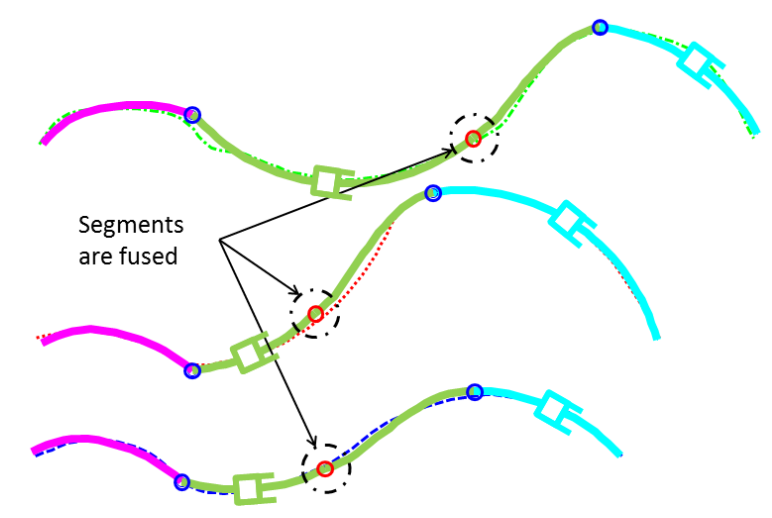

(b)

Figure 37: (a) The original chain consisted of 4 segments resulting in $E_{\max }=0.39$, yet one revolute joint exhibited limited motion. (b) The $2^{\text {nd }}$ and $3^{\text {rd }}$ segments were fused into a compound segment, resulting in a three-segment chain with $E_{\max }=0.45$. 


\subsection{Other Profile Examples}

To this point, segmentation has been exhibited on only open chain profiles. Segmentation can also be performed on closed and fixed-end profiles.

\subsubsection{Closed-Profile Example}

Two airfoils designated as E850 and E420 are segmented so that their shapes can be approximated by the closed chain of rigid-body segments [106]. This second type of profile has been investigated by Persinger et al. [79] and Zhao et al. [107]. The segmentation process is the same as for open profiles noting that the first and last points of the profiles are coincident. Figures $38 \mathrm{a}$ and $38 \mathrm{~b}$ show the two profiles and the results of the segmentation process, respectively. Figure 38c shows the simplified chain when some of the segments are fused together. The airfoils' chord lengths are normalized. The low-speed, high-lift E420 airfoil is scaled up so that the camber is longer to gain higher lift. Hence, the arc length of the profiles are $C_{1}=2.0161$ and $C_{2}=2.437$ units. For a desired piece length $s_{d}=0.0050$, the average piece lengths are $\bar{s}_{1}=0.005002$ and $\bar{s}_{2}=0.005004$ units. Again, the additional significant figures are used here to show the accuracy achieved.

The segment matrix corresponding to the final design is

$$
S M_{f}=\left[\begin{array}{llllllllll}
42 & 37 & 48 & 46 & 29 & 28 & 55 & 55 & 33 & 30 \\
42 & 37 & 48 & 46 & 64 & 77 & 55 & 55 & 33 & 30
\end{array}\right]
$$

which resulted in a maximum error of $E_{\max }=0.0425$ and the mean error, $\bar{E}=0.0123$.

\subsubsection{Fixed-End Profile Examples}

Zhao et al. [81] present applications in which the first and last points on each profile of similar length are fixed. The segmentation process for variable length profiles remains intact when fixed ends are required by merely introducing the additional constraint in Eq. (70). As an example, Fig. 39a shows three profiles with a fixed-end requirement. The arc lengths of the profiles are 


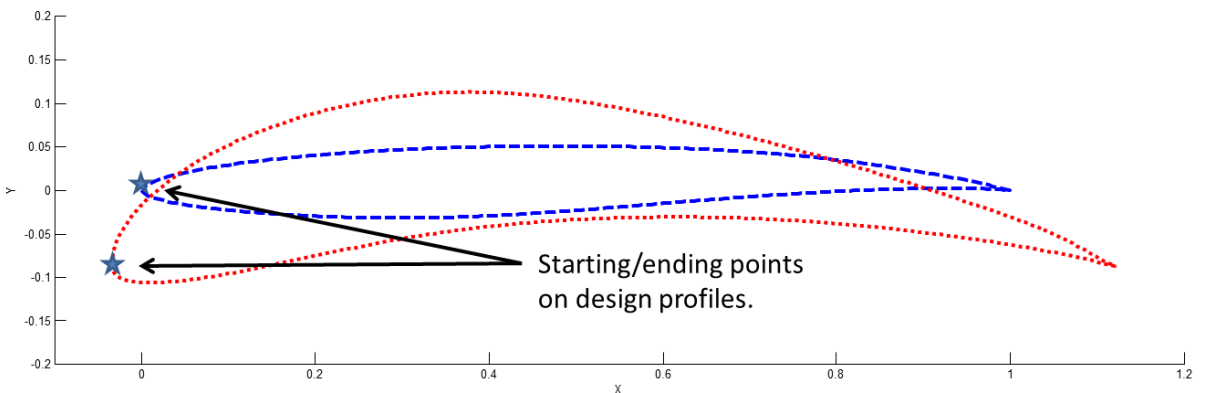

(a)

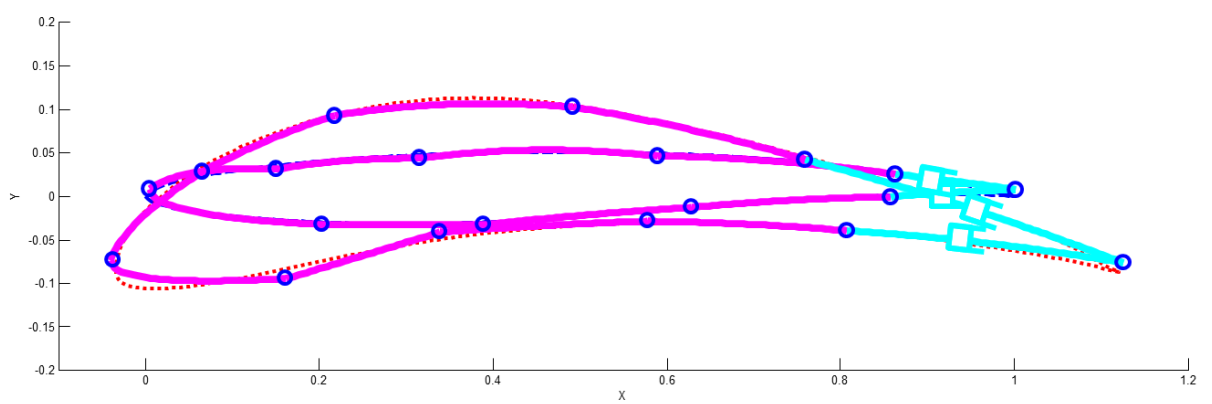

(b)

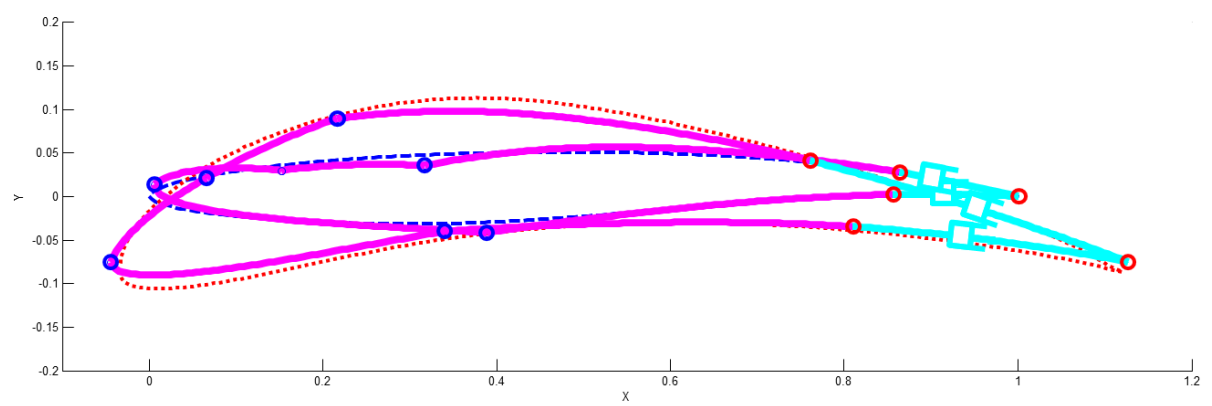

(c)

Figure 38: (a) The E420 and E850 profiles of airfoils. (b) The chain of ten segments with $E_{\max }=$ 0.015. (c) Fusing segments raises the error to $E_{\max }=0.017$ while reducing the number of joints. 
$C_{1}=12.24, C_{2}=12.90$, and $C_{3}=14.13$. A desired piece length of $s_{d}=0.1$ was specified, producing $m_{1}^{*}=122, m_{2}^{*}=129$, and $m_{3}^{*}=141$. A design vector with six segments was selected, $\mathbf{V}=[\mathscr{C} \mathscr{M} \mathscr{M} \mathscr{C} \mathscr{M} \mathscr{C}]$, and a minimum number of pieces per segment of $\alpha=8$ was designated. The final shape-approximating rigid-body chain is shown in Fig 39b, which exhibits a profile matching error of $E_{\max }=0.18$.

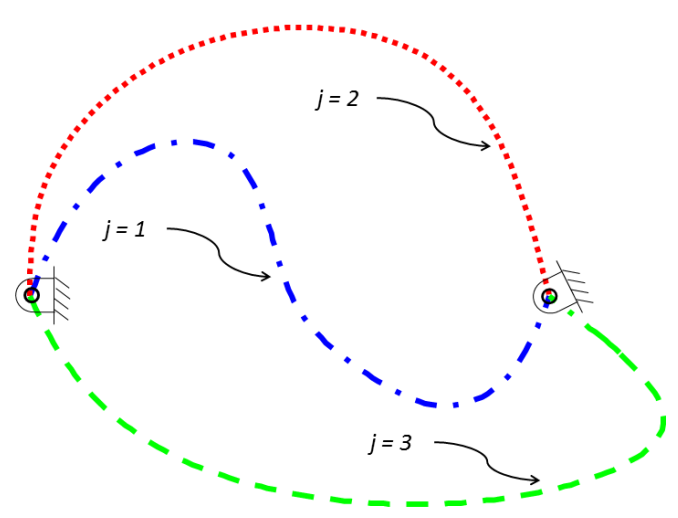

(a)

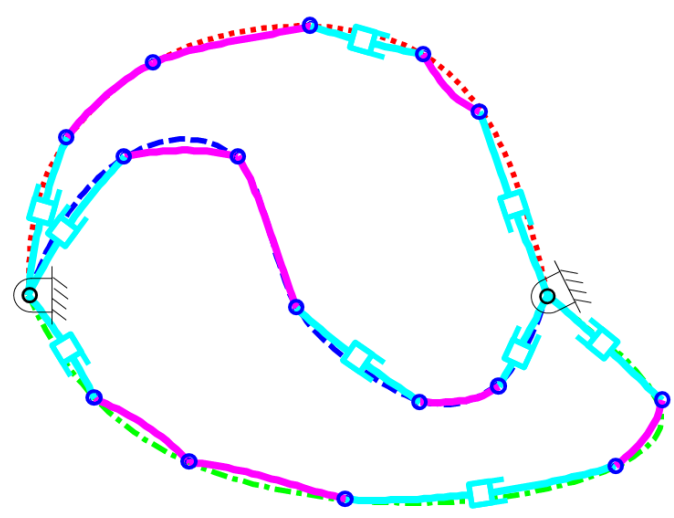

(b)

Figure 39: Fixed-end profiles shown in (a). The final, rigid-body, shape-approximating chain is shown in (b) having $E_{\max }=0.18$.

\subsection{Comparing the Number of Joints}

As noted previously, the number of segments in a chain is not equal to the number of rigid bodies it includes. An $\mathscr{M}$-segment represents one body, while a $\mathscr{C}$-segment represents two bodies connected by a $\mathrm{P}$ joint. In searching for the ideal segmentation of a set of design profiles, the number of joints in the shape approximating chain provides a better gauge for comparison than the number of segments. Generally, a chain of rigid-bodies with more joints provides a better approximation of the profiles because of the additional degrees of freedom. 
An example emphasizes the difference between segments and the associated number of rigid bodies, using the profiles in Fig. 25. Table 1 shows the results of all possible design vectors of chains with six bodies or five joints. In this case, the design with the lowest $E_{\max }$ is the fivesegment chain $[\mathscr{M} \mathscr{C} \mathscr{M} \mathscr{M} \mathscr{M}]$. Figure 40a shows this chain approximating the three profiles. Figure 40b shows the inclusion of a compound segment exhibiting lower error than the result in Fig. 40c, which shows the optimal four joint segmentation. Due to the large increase in the number of initial design vectors when compound segment types are included, the study of the associated error is left for future work.

Table 1: Design vector errors for all five-jointed chains.

\begin{tabular}{ccc}
\hline $\begin{array}{c}\text { Num. } \\
\text { Segments }\end{array}$ & $\begin{array}{c}\text { Design } \\
\text { Vector }\end{array}$ & $\begin{array}{c}\text { Max. } \\
\text { Error }\end{array}$ \\
\hline 5 & $\mathscr{M} \mathscr{C} \mathscr{M} \mathscr{M} \mathscr{M}$ & 0.2610 \\
4 & $\mathscr{M} \mathscr{C} \mathscr{M} \mathscr{C}$ & 0.3431 \\
4 & $\mathscr{M} \mathscr{C} \mathscr{C} \mathscr{M}$ & 0.4044 \\
4 & $\mathscr{C} \mathscr{C} \mathscr{M} \mathscr{M}$ & 0.5630 \\
4 & $\mathscr{M} \mathscr{M} \mathscr{C} \mathscr{M}$ & 0.6120 \\
5 & $\mathscr{M} \mathscr{M} \mathscr{C} \mathscr{M} \mathscr{M}$ & 0.6339 \\
5 & $\mathscr{M} \mathscr{M} \mathscr{M} \mathscr{C} \mathscr{M}$ & 0.6523 \\
3 & $\mathscr{C} \mathscr{C} \mathscr{C}$ & 0.7965 \\
4 & $\mathscr{C} \mathscr{M} \mathscr{M} \mathscr{C}$ & 0.9067 \\
5 & $\mathscr{M} \mathscr{M} \mathscr{M} \mathscr{M} \mathscr{C}$ & 0.9707 \\
5 & $\mathscr{C} \mathscr{M} \mathscr{M} \mathscr{M} \mathscr{M}$ & 1.0129 \\
4 & $\mathscr{C} \mathscr{M} \mathscr{M} \mathscr{C}$ & 1.3365 \\
\hline
\end{tabular}

\subsection{Versatility of the Segmentation Method}

The examples used in this dissertation are inclined to involve two or three design profiles. However, the procedures in Chapters II and III are general and apply to any number of design profiles. Figure 41a shows the seven design profiles used to express each letter in "UDAYTON." Table 2 


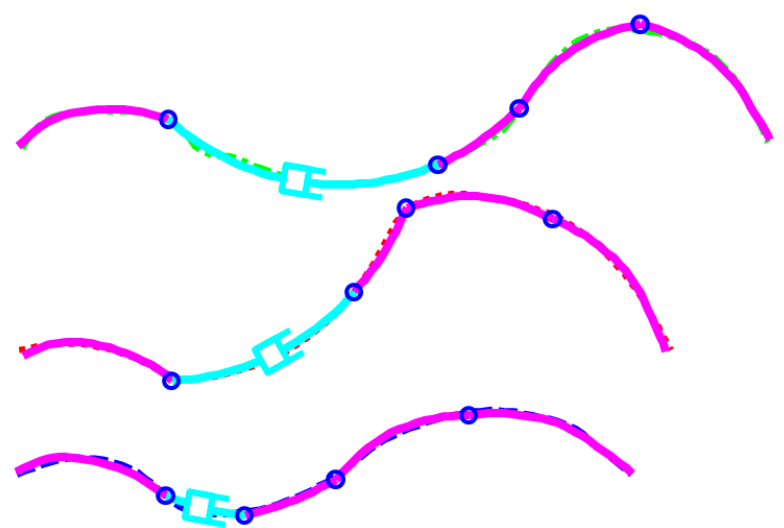

(a)

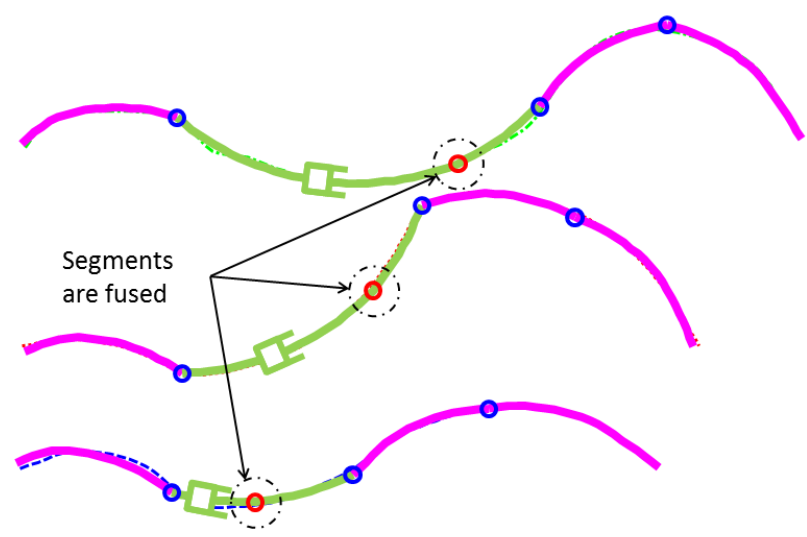

(b)

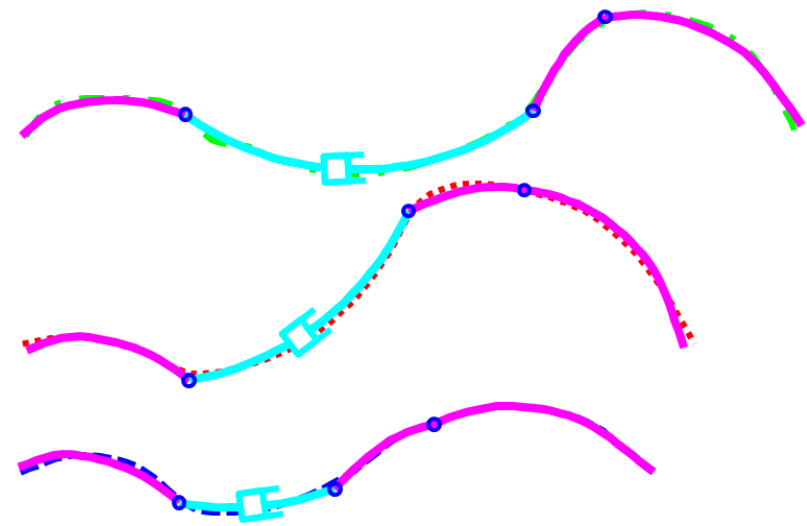

(c)

Figure 40: (a) The $[\mathscr{M} \mathscr{C} \mathscr{M} \mathscr{M} \mathscr{M}]$ chain is selected with an after-assembly error of $E_{\max }=0.26$. (b) A compound segment increases maximum error to $E_{\max }=0.28$, which is still lower than the [ $\mathscr{M} \mathscr{C} \mathscr{M} \mathscr{M}$ ] design with $E_{\text {max }}=0.37$ in (c). 
shows the arc length and number of pieces used for each letter. The result of using 15 segments is shown in Fig. $41 b$.

Table 2: Target profile data for the "UDAYTON" example shown in Fig. 41

\begin{tabular}{|c|c|c|c|c|c|c|c|}
\hline Letter & $\mathrm{U}$ & $\mathrm{D}$ & $\mathrm{A}$ & $\mathrm{Y}$ & $\mathrm{T}$ & $\mathrm{O}$ & $\mathrm{N}$ \\
\hline Arc length & 6.73 & 7.28 & 8.81 & 9.21 & 9.28 & 9.95 & 10.01 \\
\hline Num. pieces & 337 & 364 & 441 & 460 & 464 & 498 & 501 \\
\hline
\end{tabular}

\subsection{Automating the Segmentation Process}

Prismatic joints are constrained to follow a constant radius trajectory. Regions on the target profiles that have approximately constant and equal curvature are ideal for inserting a $\mathscr{C}$-segment. The constant and equal curvature criteria may be assessed by observing a plot as shown in Fig. 42 . The curvature band identifies a region in which both profiles have nearly constant curvature. Seven points from profile 1 and 35 points from profile 2 are included within the selected curvature band. This is a potential location for a $\mathscr{C}$-segment. Recalling that profile 2 is longer, the additional points included in the curvature band help to address the differences in arc length.

When specifying regions of the target profiles that will be approximated with a $\mathscr{C}$-segment, a common starting point must be selected. This ensures that the portions of the target profiles leading up to the selected point have the same arc length and can be approximated with a chain of $\mathscr{M}$-segments. Separate ending points are selected to designate the region on each profile that will be approximated with a $\mathscr{C}$-segment. The ultimate goal is to assign more points to the prismatic joint stroke on the longer profile(s). Given the sections, a single representative $\mathscr{C}$-segment may be synthesized via Eqs. (56), (57), and (58). 

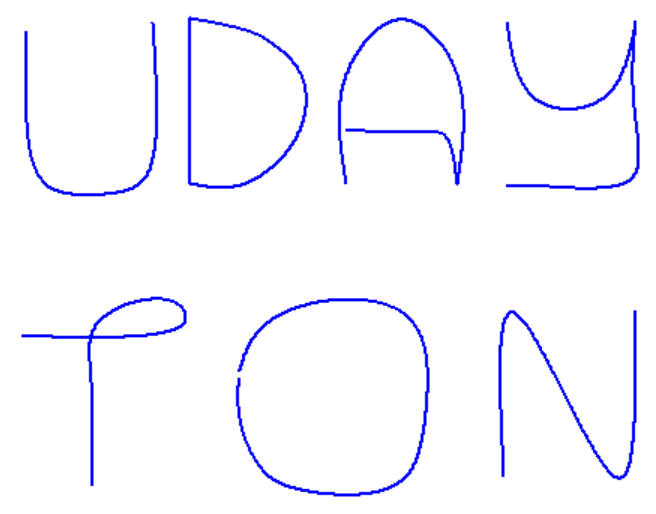

(a)
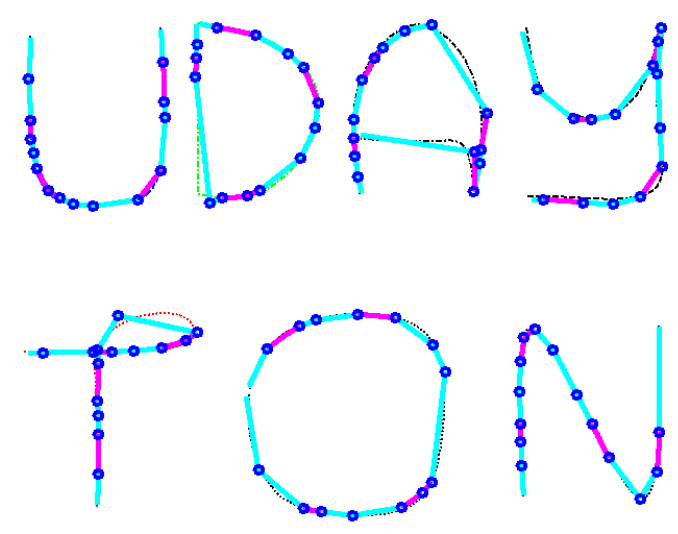

(b)

Figure 41: (a) The design profiles. (b) The [ $\mathscr{C} \mathscr{C} \mathscr{M} \mathscr{C} \mathscr{C} \mathscr{M} \mathscr{M} \mathscr{C} \mathscr{C} \mathscr{C} \mathscr{M} \mathscr{C} \mathscr{C} \mathscr{M} \mathscr{C}$ ] segmentation with $E_{\max }=0.24$. 


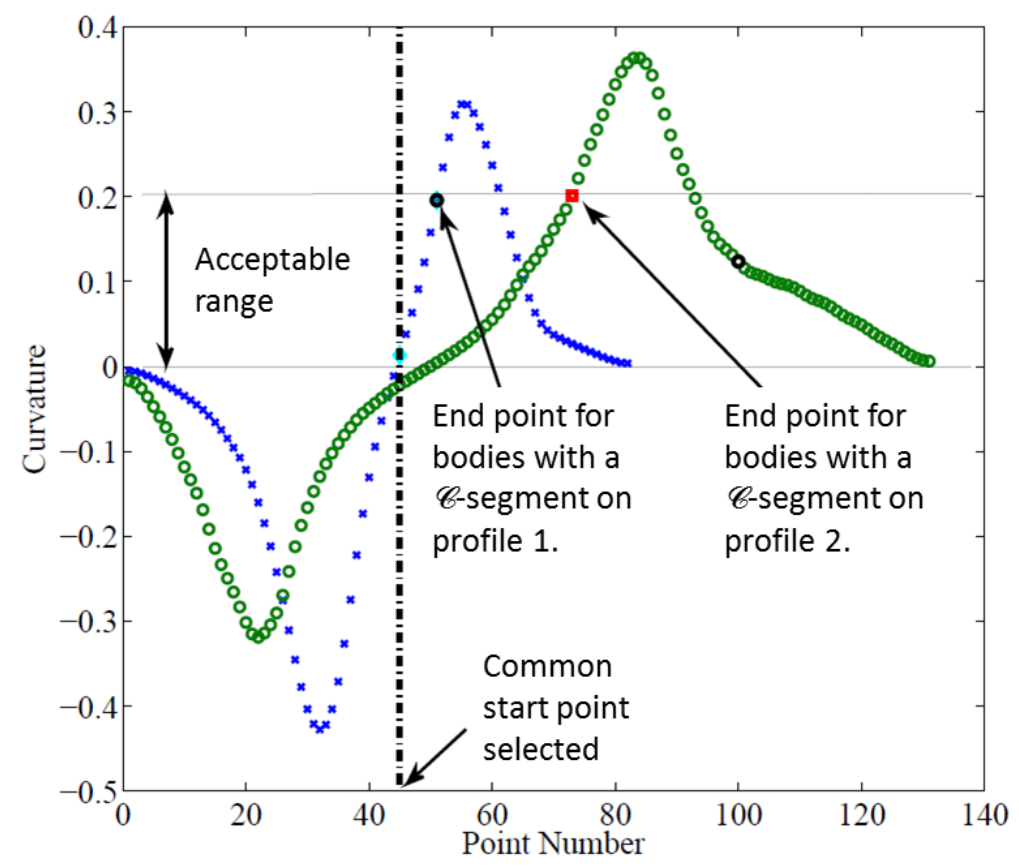

Figure 42: The range from 0 to 0.2 is an acceptable range to be approximated by an arc of constant curvature.

After identifying the first $\mathscr{C}$-segment, the arc length of the remaining portions of the profiles must be determined. As stated, if arc length differences still exist, an additional $\mathscr{C}$-segment is required. Across all target profiles, the start of the next curvature band must be the same number of points from the end of the previous curvature band. This ensures that an $\mathscr{M}$-segment, that is joined with revolute joints, can approximate the portions of profiles between curvature bands.

With each additional $\mathscr{C}$-segment, a new curvature plot that begins at the final point of the previously selected curvature band is created for each target profile. Figure 43 shows a realigned curvature plot derived from Fig. 42 after selecting the first set of curvature bands. Since profile 2 is still visibly longer than profile 1 (59 points compared to 32 points), a second curvature band must be selected for including a prismatic joint. The selection method is repeated to locate regions on each 


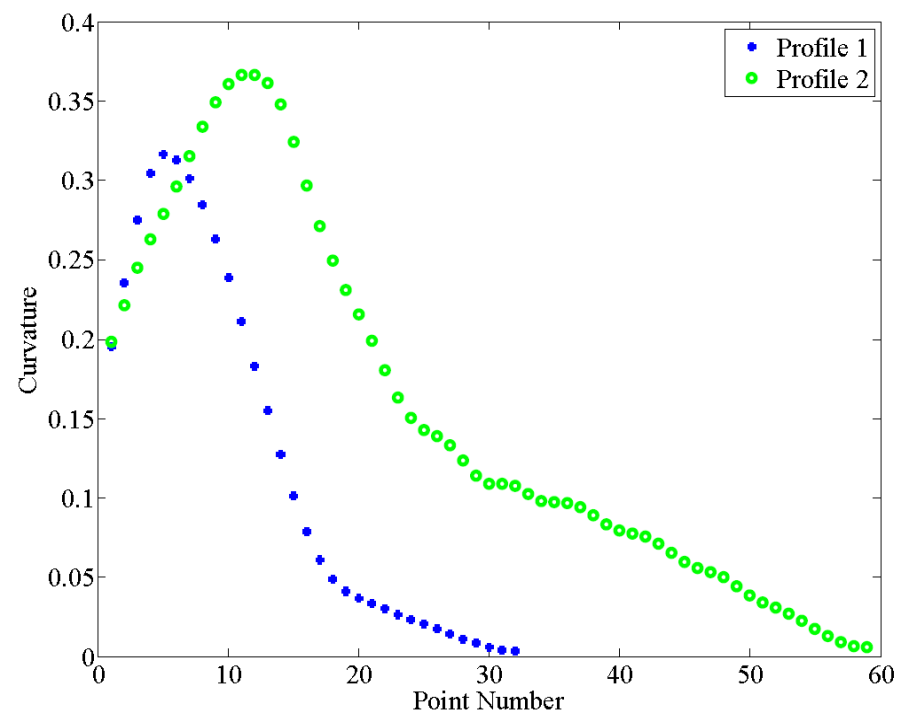

Figure 43: In order to identify a potential region in which to insert another prismatic joint, the curvature distribution (from Fig. 42) is replotted starting with the end of the previously selected curvature band.

profile where the next prismatic joint should be placed. Ultimately, $\mathscr{C}$-segments must be defined to account for all differences in the number of pieces among target profiles.

Although the beginnings of an automated process for identifying appropriate locations for $\mathscr{C}$ segments are included in this work, many challenges remain unaddressed. This includes setting constraints in the code so that ranges of relatively similar and constant curvature can be selected automatically. Furthermore, the curvature plots might undergo the smoothing process since the method requires selecting points manually. As the current method of identifying chains in Sections 3.1 to 3.3 works very effectively, the previous method of $\mathscr{C}$-segment identification was not advanced further. 


\section{CHAPTER IV}

\section{SOFTWARE IMPLEMENTATION}

The theory presented in Chapters II and III has been developed into a piece of software within MATLAB. The code, called "ShapeChanger", includes the following functions: creating and editing the design profiles, generating target profiles at user-desired levels of accuracy, and creating the error-minimizing chain of segments to approximate those profiles. Appendix A contains a step-bystep example demonstrating the use of ShapeChanger.

\subsection{Design Profiles}

Design profiles may be entered into ShapeChanger in two ways. First, design profiles may be entered as an array of points that describe a piecewise-linear curve. This would be typical of profiles generated from another application. MATLAB is then used to turn those profiles into splines. Second, design profiles may be generated directly within ShapeChanger utilizing features of MATLAB's Spline Toolbox.

The design profiles may be individually edited via the addition, deletion, or movement of the spline's control points. The design profiles may also be translated, rotated, and scaled. The placement (including orientation) of each design profile does not affect the segmentation results. The relative locations of the design profiles are critical, however, to the mechanization step of designing

a rigid-body shape-changing device. Thus, translation and rotation are present with the long-term 
goal of integrating ShapeChanger with a complete set of mechanization algorithms. The scaling option, when selected, scales all design profiles to the same arc length as the shortest profile in the set. The scaling option is useful when the design profiles need to be the same arc length, as in the case of synthesizing a chain that includes only $\mathscr{M}$-segments.

The three types of profiles identified (open, closed, and fixed-end), are all readily handled via the interface. The designer identifies the types of profiles under consideration, and the additional constraints are enforced. For example, in the case of closed profiles, the first and last points on each profile are constrained to be the same. In the case of fixed-end profiles, the first point of all profiles is constrained to be at a common point, and the last point of all profiles is constrained to be at a second common point. Finally, in the case of open profiles, no additional constraints are enforced.

\subsection{Target Profiles}

Given the arc lengths of the design profiles, target profiles may now be generated according to Eqs. (2) through (7). The accuracy of the target profiles may be gauged by their arc lengths versus those of the original design profiles. In general, decreasing the desired piece length $s_{d}$ results in a more accurate representation of the design profile. ShapeChanger does not allow a value of $s_{d}$ larger than one-third of the arc length of the shortest profile as too few pieces will result, and the likelihood of acceptable target profiles is small. Although $s_{d}$ is never exactly achieved, with a small desired piece length compared to the arc length, or $s_{d} \ll C$, the target profiles will represent the design profiles very closely. Note, however, that this is a general observation, and cases may be readily generated in which reducing $s_{d}$ does not result in an increase in the percentage of the target profile versus the design profile. One example is illustrated in Fig. 44, where approaching 500 points, the target profile's arc length still fluctuates below its design profile's arc length. The same accuracy is achieved at around $n=342$. 


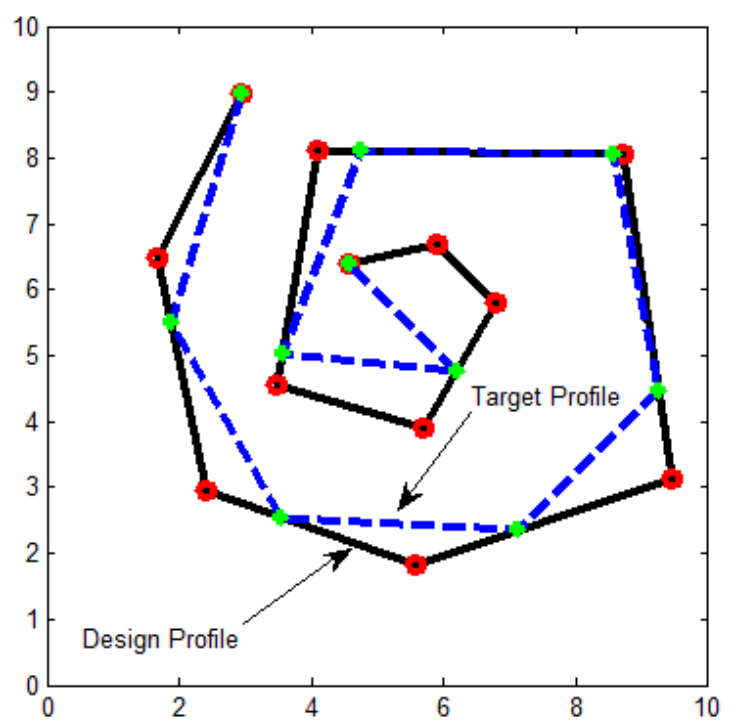

(a)

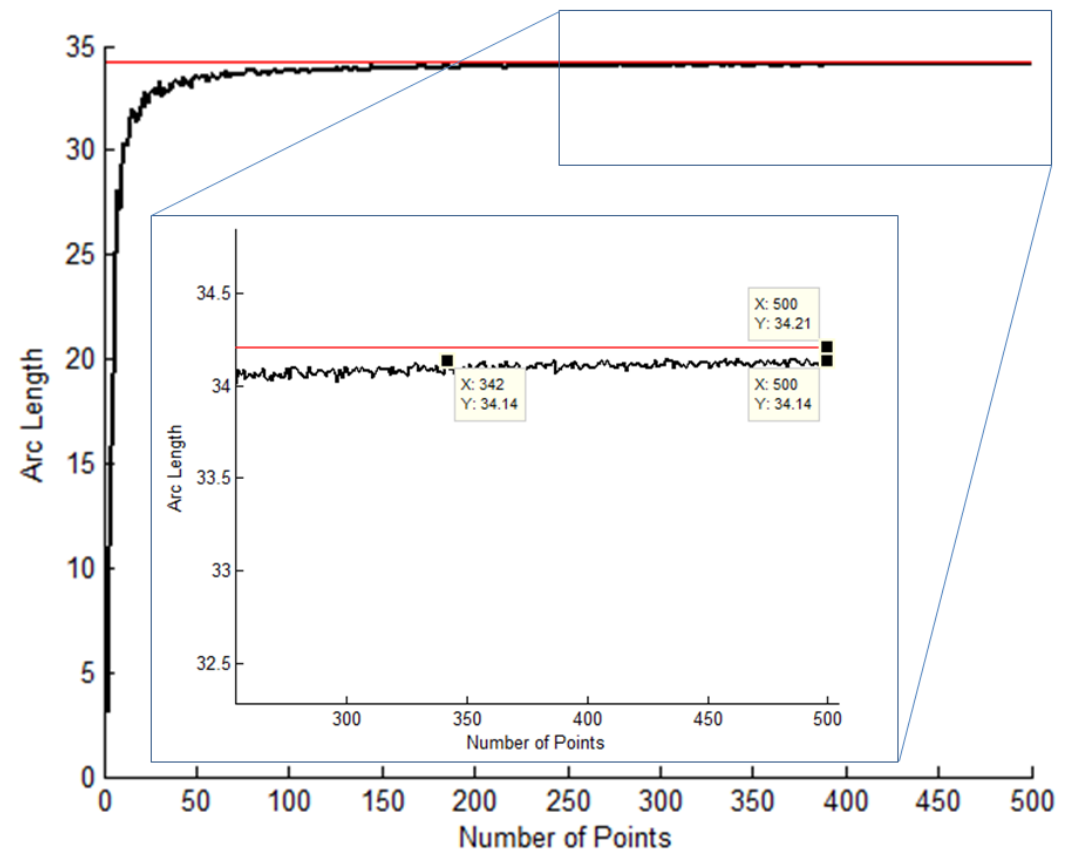

(b)

Figure 44: (a) A design profile and its corresponding target profile created with 10 points. (b) The plot of the target profile's arc length as a function of its number of points. 
Given the set of target profiles from a selected $s_{d}$, the values of $\bar{s}_{j}$ and $m_{j}^{*}$ are determined as described in Section 2.1. There is now an important interplay between variables that must be identified before segmentation may occur. The user must identify the preferred number of segments $q$ for the segmentation process as well as the minimum number of pieces per segment, $\alpha$. Note that, at a minimum, the number of pieces on the shortest profile has to be greater than $\alpha q$. The optimization algorithm adjusts the segment matrix, such that pieces can be added or removed from each segment. Thus, the heuristic that relates $q$ to the number of pieces on the shortest curve to $\alpha$ is

$$
q=\left\lfloor\frac{S_{j}}{2 \alpha}\right\rfloor, \quad j=1, \ldots, p
$$

\subsection{Segmentation}

The segmentation phase begins once acceptable target profiles have been generated that are both accurate and include the number of pieces necessary to allow for the optimization algorithm to function on the selected number of segments. Segmentation is governed by the segment matrix detailed in Eqs. (50), (51), and (52). The equations for defining a usable $S M$ are also used for the successive steps in optimizing it based on the error. The only additional computational concern is the process of generating the first instance of $S M$. Given a target profile of, for example, 100 points and 5 segments, four random integers are generated between 1 and 100. With 1 and 100, the six numbers are sorted to identify the potential list of segmentation points. Should any section have fewer than $\alpha$ pieces (or $\alpha+1$ points), the six numbers are rejected and the four integers generated anew. This is done as it is easier than creating additional functions to rectify unacceptable segment matrices. Furthermore, the computation is quick in producing another segment matrix that would fulfill the conditions. This process is repeated for the other profiles with an additional constraint. From the design vector $\mathbf{V}$, an $\mathscr{M}$ type entails that the section on that profile needs to have the same number of pieces as its corresponding section on profile 1 (shortest target profile). Hence, the 
difference in the number of pieces between any target profile and profile 1 is distributed among the type $\mathscr{C}$-sections on that profile.

The objective of optimization is to minimize the point-to-point errors among the created segments and the target profiles. The optimization constraints are the minimum number of pieces per segment $\alpha$ and the number of initial $S M$ matrices generated before the iteration is terminated. The optimization process presented can be subject to local minima. As such, multiple initial values of $S M$ are needed in search of a global minimum. Initial guesses continue to be generated and optimized until the minimum of $E_{\max }$ is not improved for five (default) additional initial values of $S M$.

The segmentation process may be run for a single design vector or set to cycle through all design vectors with a given number of segments. Under the assumption that the arc lengths of the profiles are different, a design vector must have at least one $\mathscr{C}$-segment for the above process to function. Should no $\mathscr{C}$-segments be desired, prior work, [46] for example, fully addresses that challenge. Also, a maximum number of $\mathscr{C}$-segments may be set, as their use tends to be governed by practical design limitations. Experience has shown that solving for mechanisms with $\mathscr{C}$-segments in the chain poses a greater challenge. Therefore, their inclusion in the chain design should be kept to a minimum.

There are also two concerns governing overall computation time. First, the optimization time is a function of the number of pieces in the profiles. As such, computation requirements can be reduced if the number of pieces is wisely selected. Second, if for each $\mathbf{V}$, an average of $L$ initial $S M$ matrices are attempted, then $2^{q} L$ optimizations are executed as there are $2^{q}$ possible design vectors that contain $q$ segments. Obviously, choosing fewer segments or altering the conditions that dictate the number of initial $S M$ matrices will improve computation time. 


\subsection{Visualization of Segmentation Results}

Among the features available in the software are the choices of results that are displayed. At the most detailed level, the segment optimization can be viewed. Each time an initial $S M$ is generated, another figure window opens. The optimization process shows the unconnected segments changing size until no improvement is detected. This process is the slowest option.

The second option is to view only the overall error minimizing result from each design vector, $\mathbf{V}$. When the process goes through all possible V's, the best result from each design might be attractive even though it is not the design with the least error overall. There might be other desirable qualities that are not yet included as constraints, such as the maximum number of $\mathscr{C}$-segments allowed in the chain and the implications of mechanism synthesis. Mechanizing the chain might also be easier if half of the segments are an image of the other half, i.e., the chain segments look symmetric. Nevertheless, general implications of mechanism selection on the segmentation are presented in Chapter VI.

Lastly, there is the choice to view only the overall result that displays the chain of segments corresponding to the design vector with the lowest overall error. The second option may be preferred over the third considering that the best mechanical features may not correspond to the lowest error design.

\subsection{Compound Segments}

To decide if a design is suitable for a compound segment, an angle matrix $A M$ is formulated following the concepts in Section 3.4. For the open target profile example in Fig. 37a, the joint angle matrix is

$$
A M=\left[\begin{array}{ccc}
\sigma_{1}^{1} & \sigma_{1}^{2} & \sigma_{1}^{3} \\
\sigma_{2}^{1} & \sigma_{2}^{2} & \sigma_{2}^{3} \\
\sigma_{3}^{1} & \sigma_{3}^{2} & \sigma_{3}^{3}
\end{array}\right]=\left[\begin{array}{ccc}
26.92 & -7.01 & -0.38 \\
34.99 & 5.17 & -15.10 \\
-29.53 & -6.22 & -19.36
\end{array}\right]
$$


The top row corresponds to the relative angles in degrees measured at each $\mathrm{R}$ joint in the first profile. The rows are sorted according to their arc lengths, with the shortest profile at the top. Hence, the second row lists the relative joint angles at the three $\mathrm{R}$ joints for the second longest profile. The longest profile has its relative joint angles listed in row 3. Each column, thus, represents each R joint in the three instances. Observe that by using Eq. 73, the values in the second column that represent the relative joint angles at the second $\mathrm{R}$ joint in the three instances yield $\Delta \sigma^{e}=5.17-(-7.01)=$ $12.18^{\circ}$. To create the fused segment in Fig. 37, an allowable or maximum $\sigma_{\max }$ is set at $12.2^{\circ}$, and the $\mathrm{R}$ joint is then fixed at the average relative angle of $(-7.01+5.17+(-6.22)) / 3=-2.7^{\circ}$. 


\section{CHAPTER V}

\section{SEGMENTATION AND MECHANIZATION EXAMPLES}

The theory developed in Chapters II and III is now applied to show additional examples developed utilizing the 'ShapeChanger' tool presented in Chapter IV. Many segmentation examples have already been shown in Chapter III corresponding to open, closed and fixed-end profiles. Here, another set of examples is generated to include mechanization, exhibiting the chain of rigid segments moving through all of the design profiles. The first example is a car seat that changes shape according to the size of the driver. The second example revisits the U-to-D transformation discussed in Chapter I. To demonstrate synthesis involving fixed-end profiles, a third example proposes an aircraft slat mechanism that would potentially minimize noise level. Lastly, two closed profile examples are presented. One is the transformation between two airfoils, E850 and E420, which would be beneficial as flight parameters change. Another example, a flow-field control turret design, is revisited. Discussions on mechanization techniques can be found in Section 1.3.2.

\subsection{Open Profile Examples}

Previously presented examples of open-profile designs include the following. Figure 22a shows an example of a set of open profiles. Murray et al. [46] used the open profiles in Fig. 11 that show the shape-change from a "U" to a "D." Shamsudin et al. [108] also used open profiles as an example of 
a chain of $\mathbf{V}=[\mathscr{M} \mathscr{C} \mathscr{M}]$ that approximates two different arc length design profiles. Two additional examples, both including mechanization, are now presented.

\subsubsection{Car Seat}

To illustrate the complete shape-change synthesis process, consider an automotive seat that should comfortably conform to the full range of driver sizes. According to Frey and Tecklin [109], about $80 \%$ of American adults suffer from pain in the lumbar spine during their lifetime. Musculoskeletal disorders can be caused by long periods of driving, the use of manual or stick shift transmissions, and the lack of adequate car seat adjustment [110]. Substantial research in human factors related to seat or chair design can be found in the literature [111]. Extensive human measurements at different ages can be found in [112]. These studies can be utilized to define an automotive seat profile that can be adjusted to satisfy the seating requirements for most drivers. In the design of a seat profile, Fig. 45a shows three drivers being seated relative to the steering wheel. The first profile $(j=1)$ conforms to a female from the $1^{\text {st }}$ percentile group. The second profile $(j=2)$ refers to a male from the $99^{\text {th }}$ percentile group. A mid-range profile is inserted corresponding with average-sized drivers to produce a smooth transition between the two extreme driver sizes. The intermediate profile $(j=3)$ conforms to a male from the $50^{\text {th }}$ percentile group. The three design profiles shown in Fig. 45b have significantly different lengths of both the seat and the back rest portions. These profiles have arc lengths of $C_{1}=44.42, C_{2}=51.98$ and $C_{3}=54.38$. A desired piece length is set as $s_{d}=0.35$, generating target profiles having $m_{1}^{*}=127, m_{2}^{*}=149, m_{3}^{*}=155$ pieces.

With $\alpha=12$ pieces minimum per segment, a five-segment seat was specified for the segmentation process. A typical conventional car seat has three segments, the seat, the backrest, and the headrest. Hence, for this synthesis, having the additional two segments is not an abrupt change 


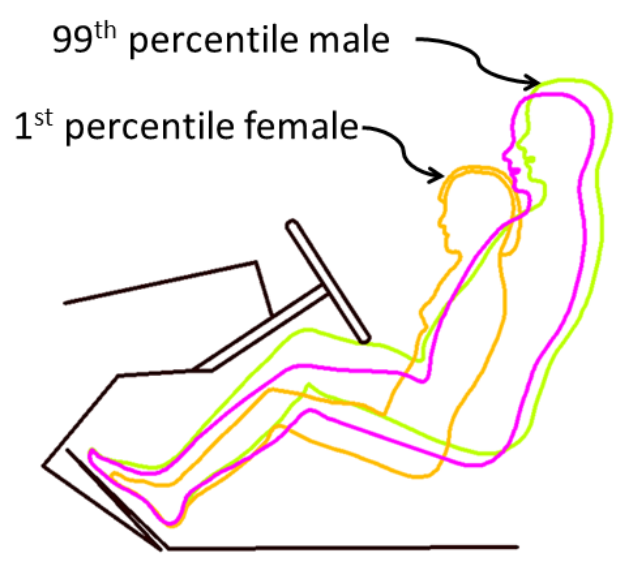

(a)

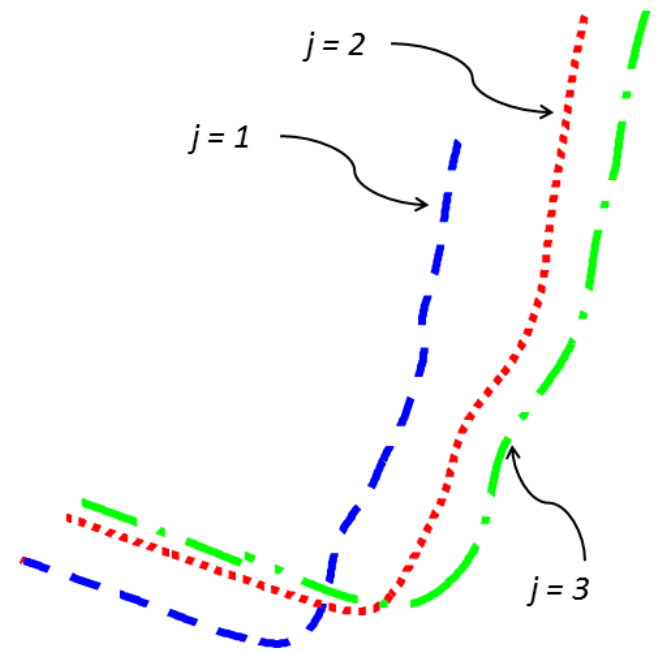

(b)

Figure 45: (a) The silhouettes of a small, an average and a large driver [112]. (b) Seat design profiles that ideally suit the small, average and large drivers from (a).

Table 3: Control points used in constructing the car seat profiles.

\begin{tabular}{|cc|cc|cc|}
\hline \multicolumn{2}{|c|}{ 1\%ile } & \multicolumn{2}{c|}{$50 \%$ ile } & \multicolumn{2}{c|}{$99 \%$ ile } \\
\hline $\mathrm{X}$ & $\mathrm{Y}$ & $\mathrm{X}$ & $\mathrm{Y}$ & $\mathrm{X}$ & $\mathrm{Y}$ \\
\hline 60.0 & 45.2 & 66.8 & 51.9 & 70.1 & 52.2 \\
59.5 & 43.0 & 66.1 & 48.7 & 69.4 & 49.8 \\
59.1 & 40.2 & 65.7 & 45.5 & 68.6 & 45.1 \\
58.6 & 37.7 & 65.2 & 42.5 & 68.4 & 43.6 \\
58.1 & 33.7 & 64.8 & 39.9 & 67.5 & 40.6 \\
57.4 & 31.2 & 64.4 & 37.2 & 67.0 & 37.1 \\
57.1 & 30.3 & 63.2 & 33.8 & 66.4 & 35.1 \\
55.9 & 28.0 & 61.3 & 31.4 & 65.5 & 33.2 \\
53.9 & 24.5 & 60.0 & 29.1 & 64.8 & 32.3 \\
53.3 & 23.1 & 59.3 & 26.6 & 63.8 & 30.9 \\
53.0 & 21.4 & 58.2 & 24.0 & 62.3 & 28.4 \\
52.6 & 20.0 & 55.9 & 20.3 & 61.3 & 24.4 \\
51.8 & 18.7 & 54.1 & 19.9 & 59.7 & 21.6 \\
50.4 & 18.1 & 51.7 & 20.5 & 57.6 & 20.3 \\
48.6 & 18.4 & 46.9 & 22.1 & 55.7 & 20.3 \\
47.3 & 18.8 & 40.5 & 24.4 & 53.6 & 20.7 \\
36.5 & 22.7 & 38.9 & 25.0 & 39.6 & 26.3 \\
\hline
\end{tabular}


from the conventional seat design. A search through every possible five-segment design vector was performed, with $\mathrm{V}=[\mathscr{C} \mathscr{M} \mathscr{M} \mathscr{M} \mathscr{C}]$ producing the lowest error approximation. This solution is logical since the notable differences between the drivers are the lengths of their thighs and the locations of their heads. Figure 46 presents the results from the segmentation process yielding $E_{\max }=0.27$ in. Further, two revolute joints exhibited minimal rotation. They were eliminated to create a compound segment within the chain as shown in Fig. $46 \mathrm{~b}$, yielding $E_{\max }=0.50$ in. This can be seen as an error increase of 0.23 in, but it is a relatively small change versus the overall size of the seat profiles.

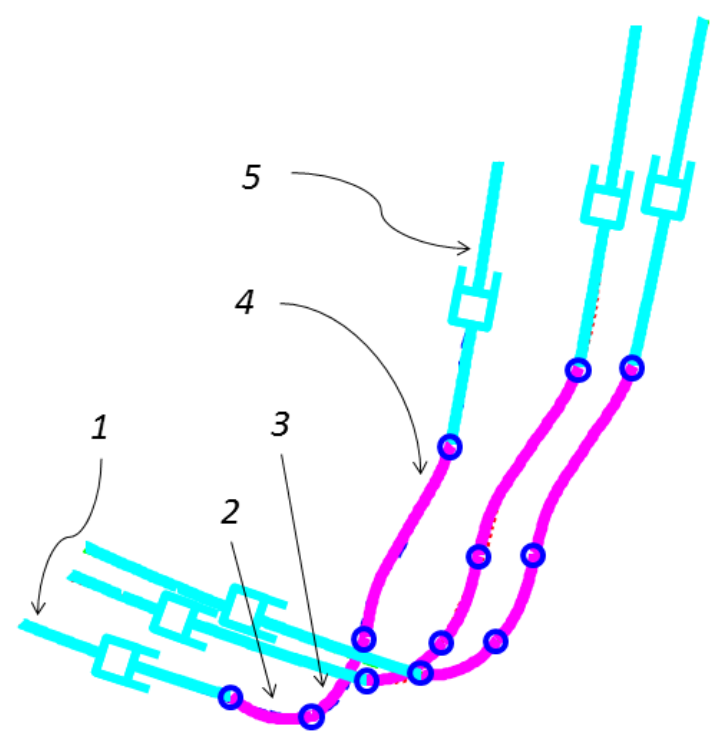

(a)

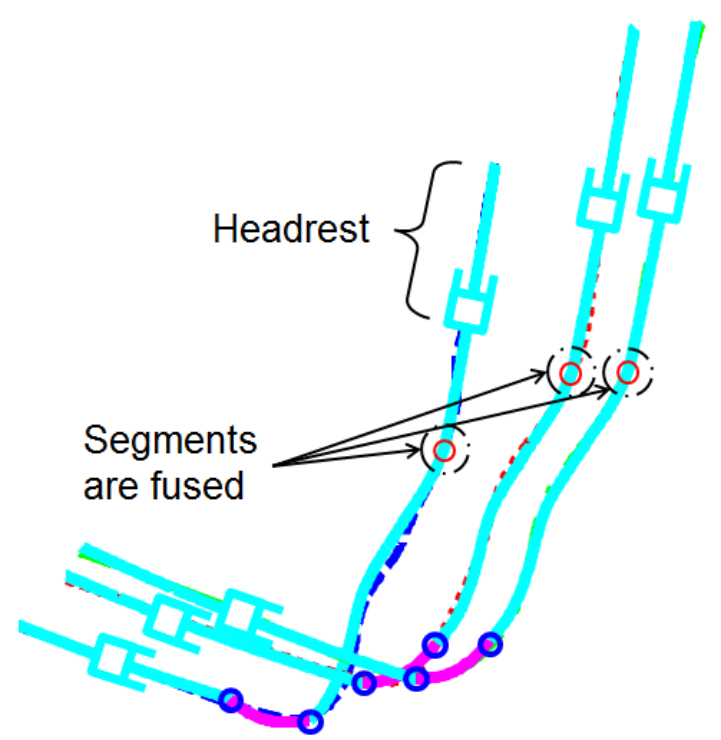

(b)

Figure 46: The three seat profiles can be approximated by the five segment shape-approximating chain in (a). The creation of a compound segment reduces the chain to three segments in (b) with only a visually-acceptable increase in error.

By using the theory of motion generation through three positions, dyads were added to reduce the DOF and form a mechanism that is able to move between the profiles. Incorporating prismatic 
joints, Figs 47 a through $47 \mathrm{c}$ show the final single-DOF mechanism that moves between the three profiles to suit the small, average, and large drivers.

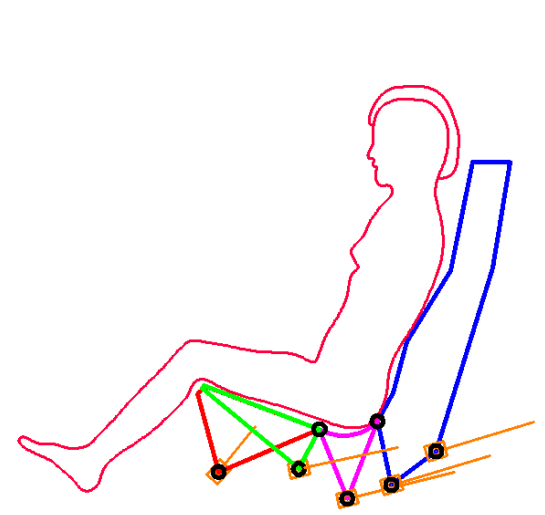

(a)

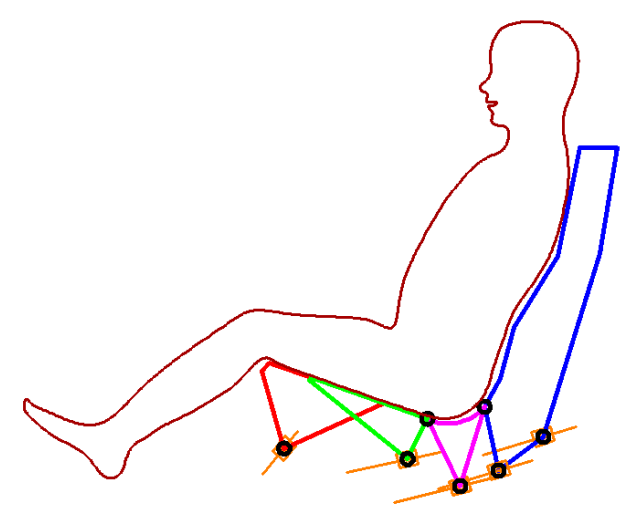

(b)

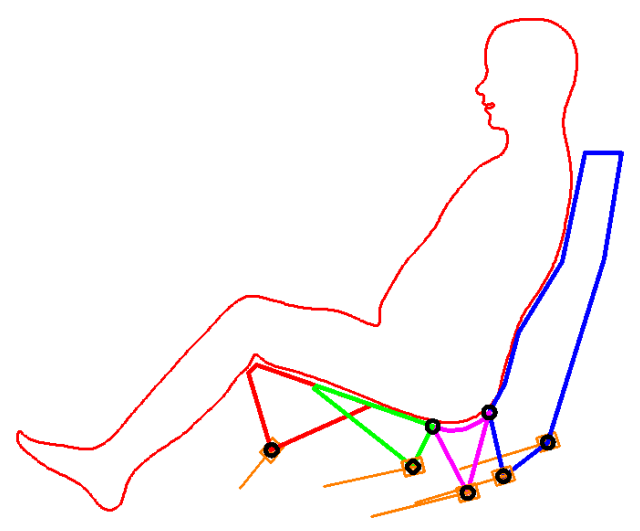

(c)

Figure 47: (a) The seat accommodates the $1^{\text {st }}$ percentile group, (b) the $50^{\text {th }}$ percentile group, and (c) the $99^{\text {th }}$ percentile group.

The detailed design of the mechanisms is defined by the pivot points shown in Fig. 48 and Tables 4 and 5 . 


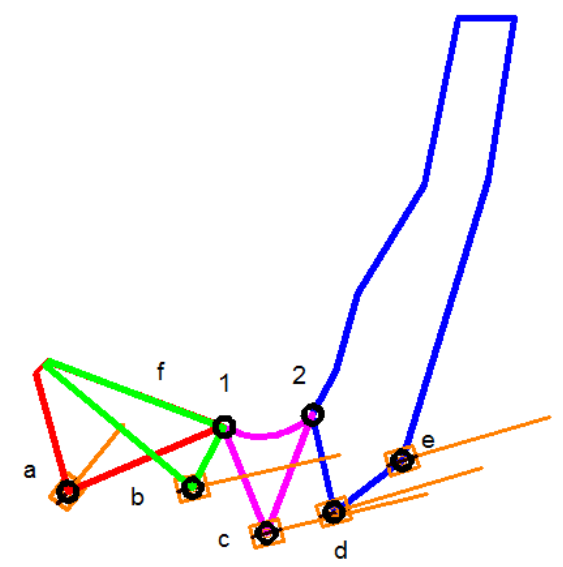

Figure 48: Labels for the dyads and revolute joints for the car seat example.

Table 4: The mechanism's revolute joints for the car seat example in the configuration shown in Fig. 48.

\begin{tabular}{|c|cc|c|}
\hline \multirow{2}{*}{ Label } & \multicolumn{2}{|c|}{ R location } & Direction \\
\cline { 2 - 3 } & $\mathrm{X}$ & $\mathrm{Y}$ & of slide \\
\hline $\mathrm{a}$ & 37.60 & 15.26 & $50.60^{\circ}$ \\
\hline $\mathrm{b}$ & 44.65 & 15.48 & $12.50^{\circ}$ \\
\hline $\mathrm{c}$ & 48.85 & 12.91 & $13.80^{\circ}$ \\
\hline $\mathrm{d}$ & 52.65 & 14.10 & $16.60^{\circ}$ \\
\hline $\mathrm{e}$ & 56.50 & 17.02 & $16.60^{\circ}$ \\
\hline $\mathrm{f}$ & 46.44 & 18.92 & $159.37^{\circ}$ \\
\hline
\end{tabular}

Table 5: The revolute joints in the segmented chain for the car seat example in the configuration shown in Fig. 48.

\begin{tabular}{|c|cc|}
\hline Joint & \multicolumn{2}{|c|}{ R location } \\
\cline { 2 - 3 } & $\mathrm{X}$ & $\mathrm{Y}$ \\
\hline 1 & 46.44 & 18.92 \\
\hline 2 & 51.44 & 19.61 \\
\hline
\end{tabular}




\subsubsection{U-to-D Transformation}

The UD mechanism originally appeared in the work of Murray et al. [46]. In that work, the letters "U" and "D", as shown in Fig. 49a, had similar arc lengths. Thus, a chain of only $\mathscr{M}$ segments was sufficient to approximate the two profiles. For this work, new "U" and "D" profiles are used that have significantly different arc lengths. The new target profiles are shown in Fig. 49b. The arc length of " $\mathrm{U}$ " is $C_{1}=37.58$ units and of " $\mathrm{D}$ " is $C_{2}=45.79$. These profiles more closely approximate the actual relative size of capital letters belonging to the same font. Table 6 shows the coordinates used in constructing the two letters.

Table 6: Control points used in constructing the $\mathrm{U}$ and $\mathrm{D}$ profiles.

\begin{tabular}{|cc|cc|}
\hline \multicolumn{2}{|c|}{$\mathrm{U}$} & \multicolumn{2}{c|}{$\mathrm{D}$} \\
\hline $\mathrm{X}$ & $\mathrm{Y}$ & $\mathrm{X}$ & $\mathrm{Y}$ \\
\hline 1.12 & 18.68 & 4.35 & 16.70 \\
1.12 & 15.87 & 4.31 & 11.59 \\
1.09 & 12.64 & 4.29 & 6.66 \\
1.09 & 9.92 & 4.24 & 4.27 \\
1.09 & 7.55 & 4.11 & 1.80 \\
1.12 & 6.78 & 4.27 & 0.20 \\
1.21 & 6.15 & 5.57 & 0.12 \\
1.81 & 4.80 & 10.27 & 0.66 \\
3.06 & 3.46 & 10.97 & 1.08 \\
4.77 & 2.80 & 12.18 & 2.66 \\
6.39 & 2.62 & 13.07 & 5.06 \\
8.15 & 3.22 & 13.33 & 9.21 \\
9.35 & 4.38 & 12.92 & 12.21 \\
10.19 & 5.58 & 12.31 & 13.90 \\
10.46 & 12.25 & 10.22 & 15.86 \\
10.49 & 13.90 & 8.14 & 16.65 \\
10.46 & 16.29 & 5.35 & 16.76 \\
10.40 & 18.74 & 4.35 & 16.70 \\
\hline
\end{tabular}




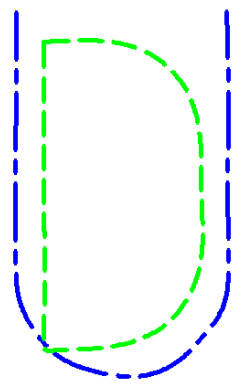

(a)

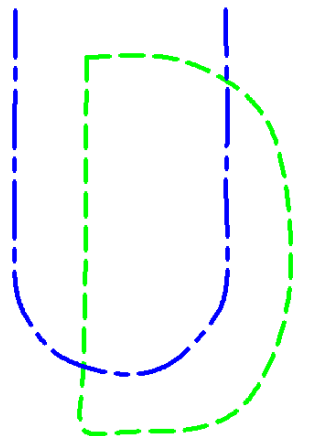

(b)

Figure 49: (a) The same arc length design profiles for $U$ and D. (b) The new profiles include an enlarged " $D$ " so that its overall size is comparable to that of "U."

The segmentation result shown in Fig. 49 b illustrates the match between a chain of $[\mathscr{C} \mathscr{M} \mathscr{C}$ $\mathscr{M} \mathscr{M}]$ segments and the target profiles, with a maximum point-to-point error $E_{\max }=0.27$. The number of $\mathscr{C}$-segments is limited to two due to the modest difference in arc length. It is desirable that the extension of each $\mathscr{C}$-segment is limited to 3 times its shortest length. To mechanize this chain, GCP techniques were implemented and the results assembled in AutoCAD. There are three prismatic joints included in the mechanism since their moving pivots lie on straight lines. This approach was inspired by the work of Zhao et al. [107]. Another inspiration is the work of Perkins et al. [82] that highlights the use of a prismatic input as a way to avoid kinematic singularities. Since shape-changing mechanisms involve multiple rigid bodies, incorporating prismatics may be advantageous in the synthesis of a working mechanism. Figures 51a through 51d show the mechanism as it progresses from approximating the "U" to the "D." The detailed design of the mechanism is defined by the pivot points shown in Fig. 52 and Tables 7 and 8 . 


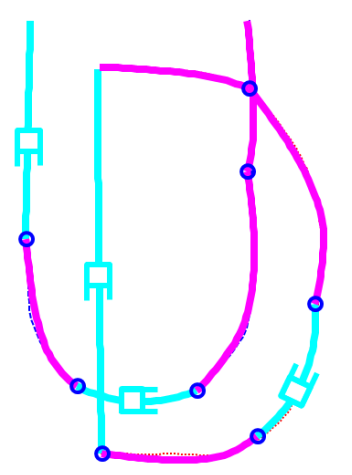

Figure 50: Segmentation shows good approximations with a maximum point-to-point error $E_{\max }=$ 0.27 .

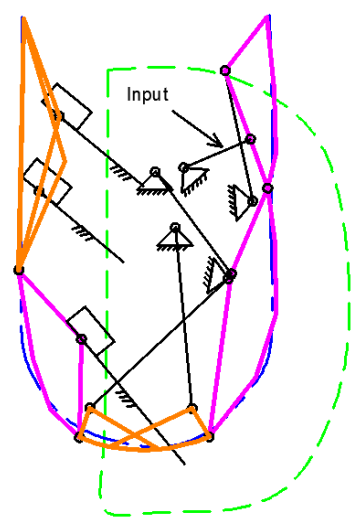

(a)

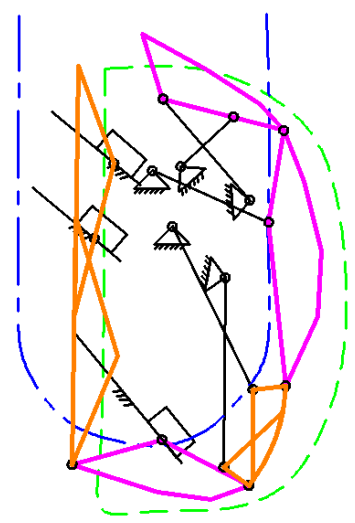

(c)

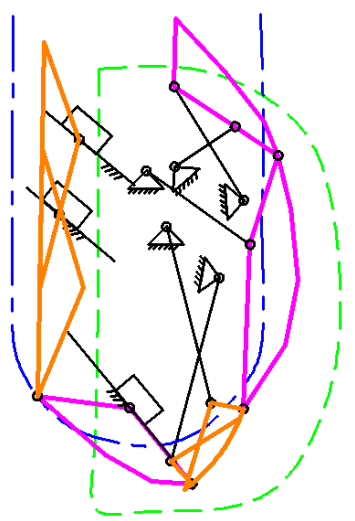

(b)

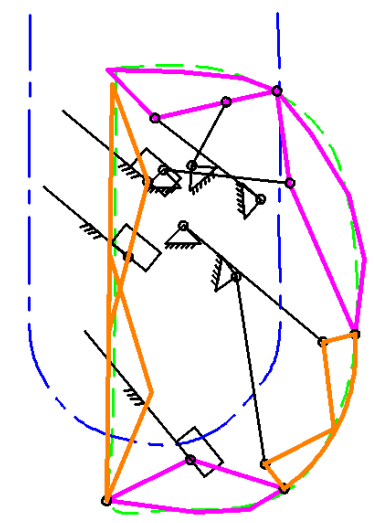

(d)

Figure 51: (a) The mechanism aligned with the "U." (b) An interim position close to the "U." (c) An interim position close to the "D." (d) The mechanism aligned with the "D." 


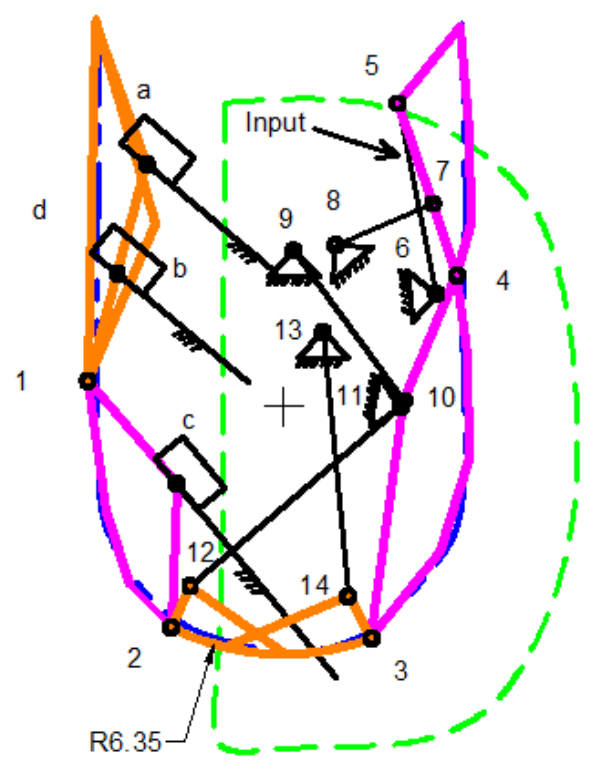

Figure 52: Labels for the dyads and revolute joints for the UD example.

Table 7: The mechanism's revolute joints for the UD example in the configuration shown in Fig. 52.

\begin{tabular}{|c|cc|c|}
\hline \multirow{2}{*}{ Label } & \multicolumn{2}{|c|}{ R location } & Direction \\
\cline { 2 - 3 } & $\mathrm{X}$ & $\mathrm{Y}$ & of slide \\
\hline $\mathrm{a}$ & 2.34 & 15.12 & $-39.75^{\circ}$ \\
\hline $\mathrm{b}$ & 1.64 & 12.27 & $-39.45^{\circ}$ \\
\hline $\mathrm{c}$ & 3.12 & 6.92 & $-50.57^{\circ}$ \\
\hline $\mathrm{d}$ & 0.83 & 9.56 & $88.70^{\circ}$ \\
\hline
\end{tabular}


Table 8: The revolute joints in the segmented chain for the UD example in the configuration shown in Fig. 52.

\begin{tabular}{|c|cc|}
\hline \multirow{2}{*}{ Joint } & \multicolumn{2}{|c|}{ R location } \\
\cline { 2 - 3 } & $\mathrm{X}$ & $\mathrm{Y}$ \\
\hline 1 & 0.83 & 9.56 \\
\hline 2 & 2.96 & 3.25 \\
\hline 3 & 8.10 & 2.97 \\
\hline 4 & 10.29 & 12.27 \\
\hline 5 & 8.77 & 16.68 \\
\hline 6 & 9.76 & 11.80 \\
\hline 7 & 9.67 & 14.11 \\
\hline 8 & 7.18 & 13.05 \\
\hline 9 & 6.11 & 12.96 \\
\hline 10 & 8.93 & 9.06 \\
\hline 11 & 8.84 & 8.90 \\
\hline 12 & 3.45 & 4.31 \\
\hline 13 & 6.86 & 10.82 \\
\hline 14 & 7.50 & 4.05 \\
\hline
\end{tabular}

\subsection{Fixed-End Example}

The goal of this example is to apply the shape-change methodology to fixed-end profiles and devise a design that replaces the conventional slat mechanism for a wing. A set of fixed-end profiles is defined by the fact that the location of the first point of all profiles is in a fixed location, whereas the location of the last point of all profiles is in a separate fixed location. Consider the motion of a slat (or leading edge flap) portion of the NASA wing airfoil 30P30N [113] shown in Figure 53. A conventional slat mechanism is shown in Fig. 54 [114]. An input shaft (not shown) can rotate the two pinions (parts 10 and 11) that have different radii. Then the slat is deployed forward by one drive arm attached to a rack and rotated by the push of another faster moving drive arm attached to a different rack. 


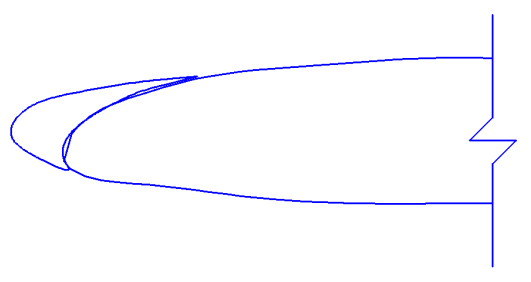

(a)

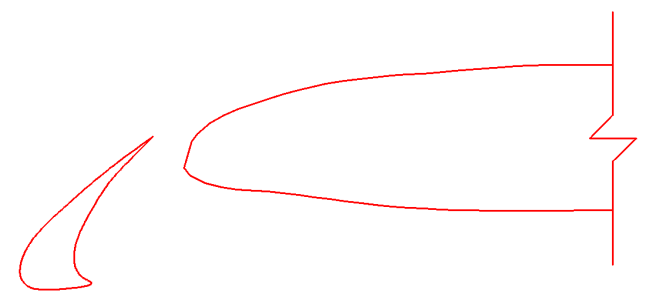

(b)

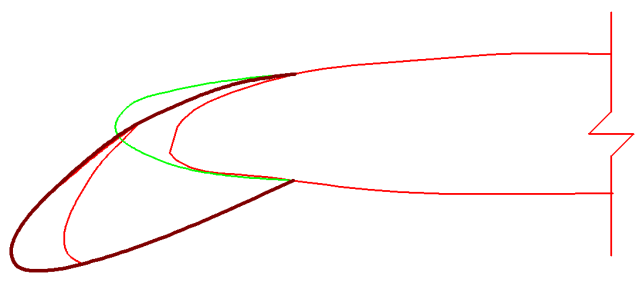

(c)

Figure 53: (a) The NASA 30P30N wing is shown with its slat in the stowed configuration. (b) The slat in the deployed configuration. (c) The slat locations have been used to identify a fixed-end design challenge.

In Fig. 55a, Khorrami reported that the trailing edge or tip of the slat is the location of very high sound pressure, which can be reduced by about $20 \mathrm{~dB}$ with the treatment of special porous material at that region [115]. Hence, eliminating air flow between the slat and the main (fixed) wing segment is a viable solution to this problem. However, further investigations are needed to confirm this as well as to establish its influence on increasing lift while reducing drag.

A proposed design changes the type of slat from split type to one that is hinged to the main wing airfoil. Abbott and Von Doenhoff [116] discussed hinged slats such as those shown in Fig. 55b. This type of hinged slat is also used in the research work of Kota et al. [5] and Santer et al. [13]. A benefit of covering the gap between the slat and the main airfoil is the potential for noise reduction. Swift reported some findings that related aircraft noise to, among others, cardiovascular, sleeping, and 

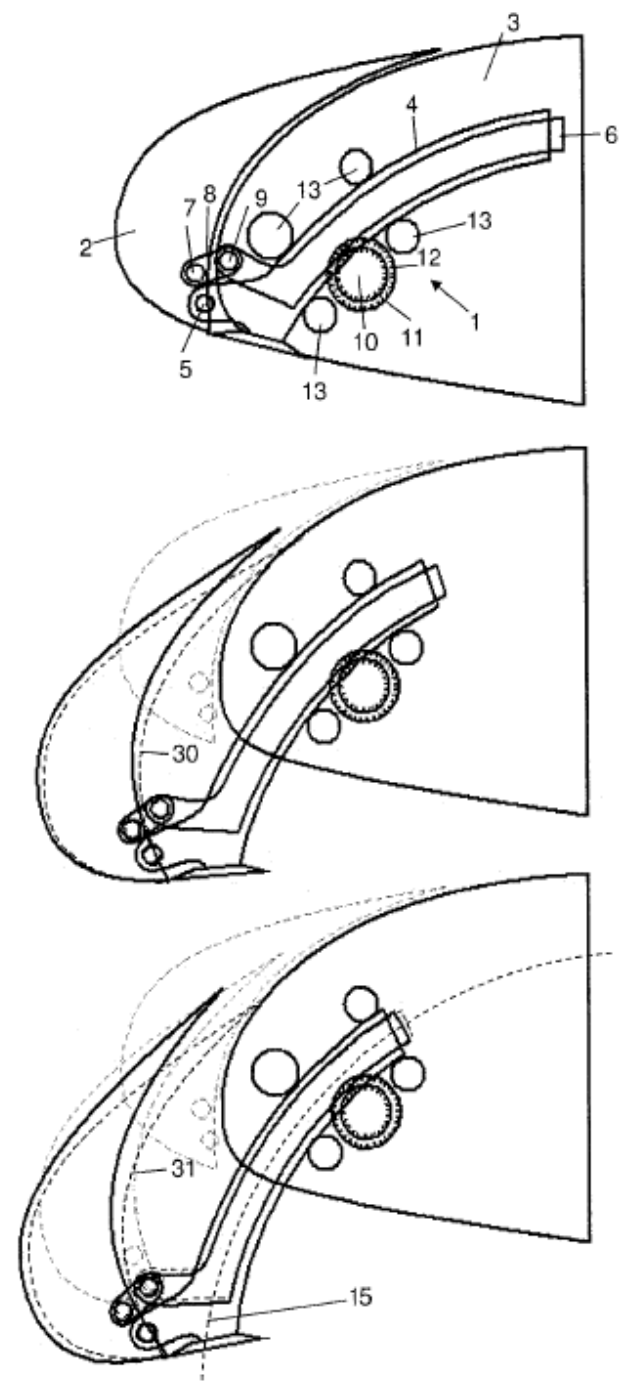

Figure 54: The two rack and pinion systems drive the slat out of the main wing [114]. 


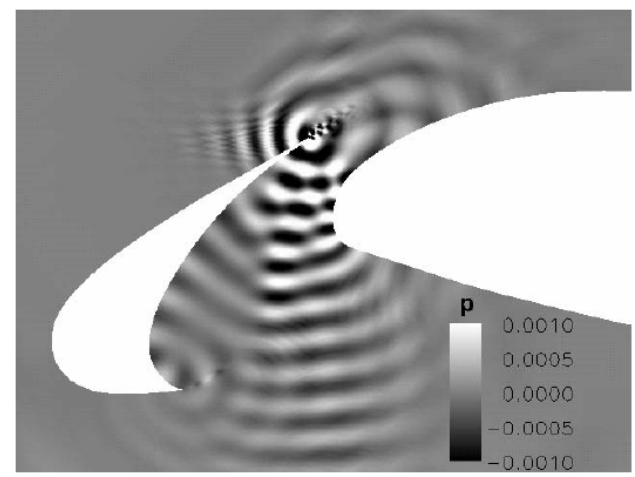

(a)
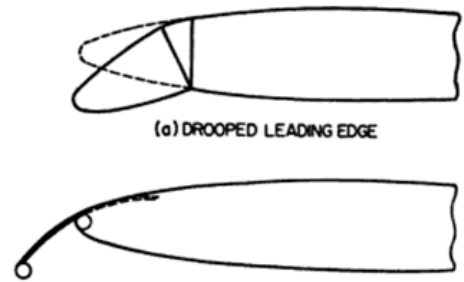

(b) UPPER SURFACE LEADING EDGE FLAP
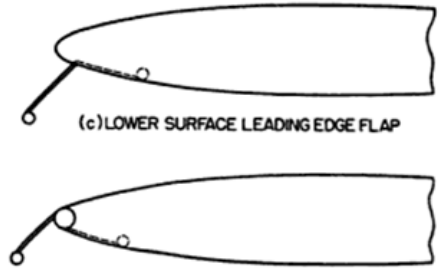

(d) FLAP HINGED ABOUT LEADING EDGE RADIUS

(b)

Figure 55: (a) Fluctuating instantaneous sound pressure field [115]. (b) Many early types of slats are hinged to the main wing element [116].

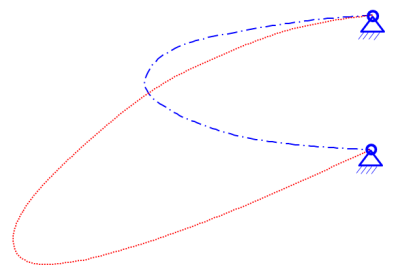

(a)

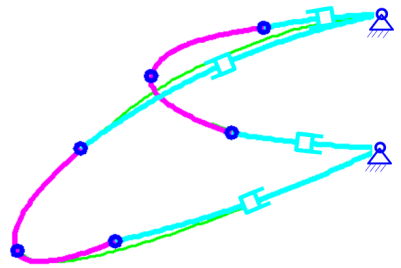

(b)

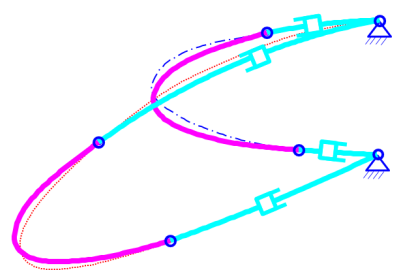

(c)

Figure 56: (a) The target profiles generated with $s_{d}=0.1$. (b) The four-segment design has an $E_{\max }=0.14$. (c) The error minimizing segmentation result with a $\left.\mathscr{C} \mathscr{M} \mathscr{C}\right]$ chain and $E_{\max }=$ 0.16 . 
annoyance problems especially among people who live in the vicinity of an airport [117]. The data points for the new slat design are listed in Table 9.

Table 9: Control points that define the stowed and deployed profiles of the wing slat.

\begin{tabular}{|cc|cc|}
\hline \multicolumn{2}{|c|}{ Stowed } & \multicolumn{2}{c|}{ Deployed } \\
\hline $\mathrm{X}$ & $\mathrm{Y}$ & $\mathrm{X}$ & $\mathrm{Y}$ \\
\hline 9.33 & 8.92 & 9.33 & 8.92 \\
8.95 & 8.89 & 8.83 & 8.85 \\
8.57 & 8.86 & 8.33 & 8.75 \\
8.20 & 8.81 & 7.84 & 8.62 \\
7.82 & 8.76 & 7.37 & 8.44 \\
7.45 & 8.69 & 6.90 & 8.23 \\
7.08 & 8.62 & 6.45 & 8.01 \\
6.72 & 8.53 & 6.02 & 7.74 \\
6.37 & 8.39 & 5.61 & 7.44 \\
6.10 & 8.13 & 5.23 & 7.11 \\
6.07 & 7.79 & 4.86 & 6.77 \\
6.36 & 7.55 & 4.50 & 6.41 \\
6.70 & 7.38 & 4.20 & 6.00 \\
7.05 & 7.25 & 4.12 & 5.52 \\
7.41 & 7.15 & 4.54 & 5.31 \\
7.78 & 7.08 & 5.04 & 5.34 \\
8.16 & 7.04 & 5.53 & 5.46 \\
8.54 & 7.00 & 6.02 & 5.60 \\
8.91 & 6.98 & 6.50 & 5.76 \\
9.29 & 6.96 & 6.97 & 5.94 \\
& & 7.44 & 6.13 \\
& & 7.91 & 6.33 \\
& & 8.37 & 6.54 \\
& & 8.83 & 6.74 \\
& & 9.29 & 6.96 \\
\hline
\end{tabular}

The new design starts with the target profile generation and segmentation as shown in Figure 56. Analysis of the $[\mathscr{C} \mathscr{M} \mathscr{M} \mathscr{C}]$ design shows that the change in joint angle between the two $\mathscr{M}$ segments is insignificant. Hence, the slat can be formed by fusing the second and third segments into a single segment, thus reducing the chain to $[\mathscr{C} \mathscr{M} \mathscr{C}$ ]. Figures 57 a through $57 \mathrm{~d}$ show the movement 
of the slat chain by a single-DOF system. The input is the dyad link indicated in Fig. 57a, which can also be connected to a shorter crank link (not shown) that is able to complete a full revolution and serve as the input. This input dyad is short, moves monotonically, and is closest to the main actuator that is likely to be situated in the main wing element. The detailed design of the mechanism

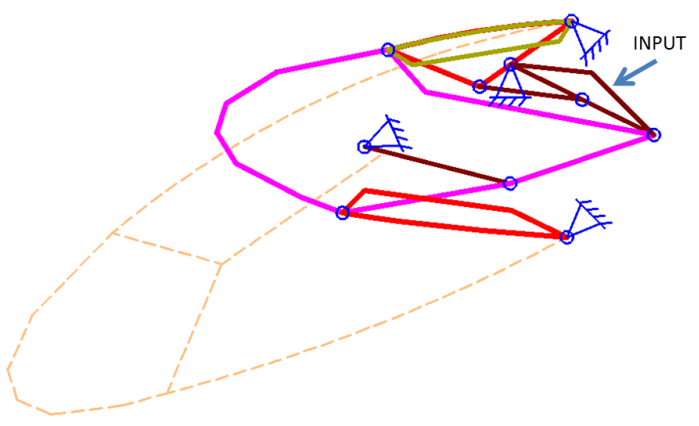

(a)

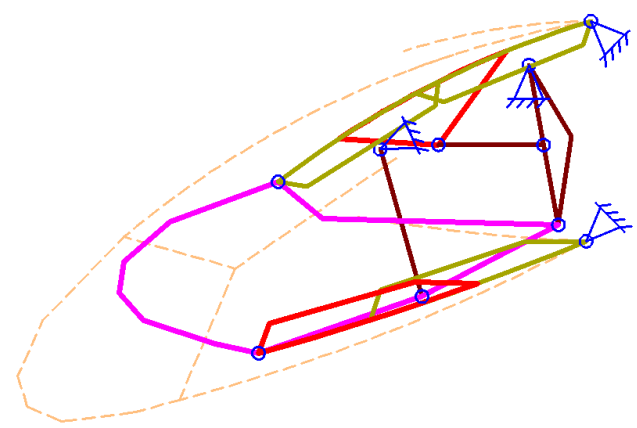

(c)

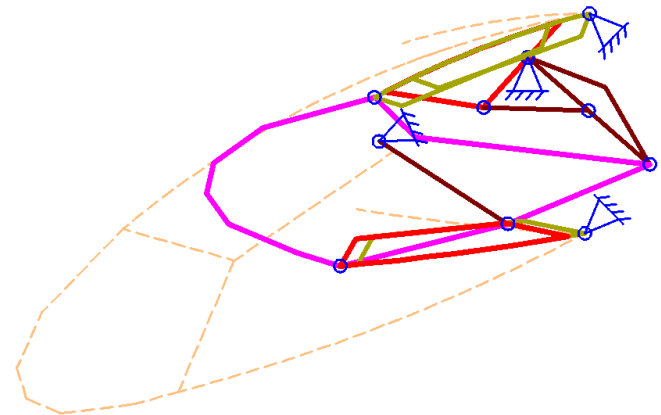

(b)

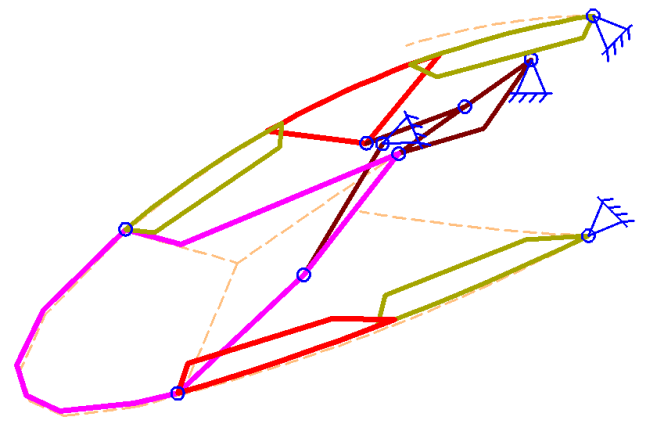

(d)

Figure 57: (a) A fully stowed slat. (b) The slat starts to deploy. (c) An interim position as it approaches the deployed position. (d) The fully deployed (extended) slat.

is defined by the pivot points as shown in Fig. 58 and Table 10. 


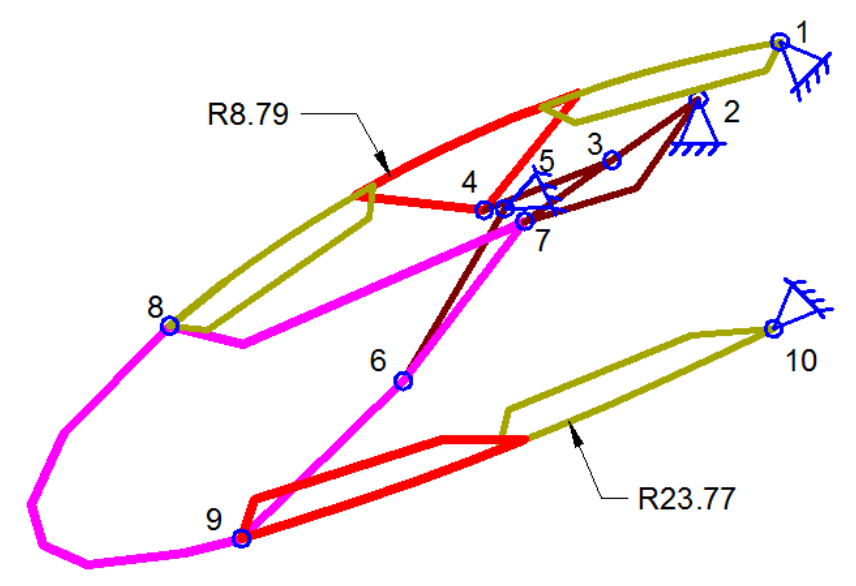

Figure 58: Labels for the dyads and revolute joints for the wing slat example.

Table 10: The revolute joints in the segmented chain for the wing slat example in the configuration shown in Fig. 58.

\begin{tabular}{|c|cc|}
\hline \multirow{2}{*}{ Joint } & \multicolumn{2}{|c|}{ R location } \\
\cline { 2 - 3 } & $\mathrm{X}$ & $\mathrm{Y}$ \\
\hline 1 & 9.33 & 8.92 \\
\hline 2 & 8.77 & 8.53 \\
\hline 3 & 8.18 & 8.11 \\
\hline 4 & 7.31 & 7.77 \\
\hline 5 & 7.45 & 7.78 \\
\hline 6 & 6.76 & 6.60 \\
\hline 7 & 7.59 & 7.69 \\
\hline 8 & 5.16 & 6.98 \\
\hline 9 & 5.65 & 5.52 \\
\hline 10 & 9.29 & 6.96 \\
\hline
\end{tabular}




\subsection{Closed-Loop Example}

A set of closed profiles are defined by the fact that the locations of the first and last points of a profile are in the same location. The two examples studied are the morphing wing airfoils and the flow-field control.

\subsubsection{Morphing Wing}

The wing airfoils introduced in Section 3.5.1 are revisited to mechanize the shape-change. Figure 38 shows the profiles and the results of segmentation. Although the normalized data of the airfoils are available from sources such as [106], the actual sizes are not known. Here, the E420 high-lift airfoil is scaled-up in order to have longer camber that helps to attain higher lift. The data points used for this synthesis are shown in Table 11.

The resulting target profiles have dissimilar arc lengths $\left(C_{1}=2.02\right.$ and $\left.C_{2}=2.4\right)$. Note that in Fig 38, the two profiles overlap such that the mechanism can be assembled inside the airfoils. Figure 59 shows the chosen profiles and the progression from high-lift airfoil to high-speed airfoil. The two profiles are positioned as such in hope to have all mechanism dyads placed within the common region. The system produced is one DOF and may be driven by an actuator or a motor on a dyad link that progresses monotonically, as shown in Fig. 59b. Any other dyad works well to actuate this mechanism. The detailed design of the mechanisms is defined by the important pivot points as shown in Fig. 60 and Tables 12, 13, and 14.

\subsubsection{Flow-Field Control}

A set of closed profiles are defined by the shared location of the first and last points of each profile. Consider the flow-field turret discussed by Persinger et al. in [79]. Figure 61a shows the 
Table 11: Points used in constructing the E850 and E420 profiles.

\begin{tabular}{|cc|cc||cc|cc|}
\hline \multicolumn{2}{|c|}{ E850 } & \multicolumn{2}{c||}{ E420 } & \multicolumn{2}{c|}{ E850 } & \multicolumn{2}{c|}{ E420 } \\
\hline $\mathrm{X}$ & $\mathrm{Y}$ & $\mathrm{X}$ & $\mathrm{Y}$ & $\mathrm{X}$ & $\mathrm{Y}$ & $\mathrm{X}$ & $\mathrm{Y}$ \\
\hline 1 & 0 & 1.12 & -0.09 & 0.02 & 0.01 & 0 & -0.02 \\
0.98 & 0.01 & 1.1 & -0.07 & 0 & 0 & -0.02 & -0.05 \\
0.95 & 0.01 & 1.07 & -0.06 & 0.02 & -0.01 & -0.03 & -0.07 \\
0.93 & 0.02 & 1.04 & -0.05 & 0.04 & -0.02 & -0.03 & -0.1 \\
0.91 & 0.02 & 1.02 & -0.04 & 0.07 & -0.02 & 0 & -0.11 \\
0.88 & 0.02 & 0.99 & -0.03 & 0.09 & -0.02 & 0.03 & -0.11 \\
0.83 & 0.03 & 0.93 & -0.01 & 0.11 & -0.02 & 0.06 & -0.1 \\
0.79 & 0.04 & 0.88 & 0.01 & 0.14 & -0.03 & 0.09 & -0.1 \\
0.74 & 0.04 & 0.82 & 0.03 & 0.16 & -0.03 & 0.11 & -0.09 \\
0.72 & 0.04 & 0.8 & 0.03 & 0.19 & -0.03 & 0.14 & -0.09 \\
0.67 & 0.05 & 0.74 & 0.05 & 0.21 & -0.03 & 0.17 & -0.08 \\
0.64 & 0.05 & 0.71 & 0.06 & 0.26 & -0.03 & 0.23 & -0.07 \\
0.6 & 0.05 & 0.66 & 0.07 & 0.31 & -0.03 & 0.29 & -0.06 \\
0.55 & 0.05 & 0.6 & 0.08 & 0.35 & -0.03 & 0.34 & -0.05 \\
0.5 & 0.05 & 0.54 & 0.1 & 0.4 & -0.03 & 0.4 & -0.04 \\
0.45 & 0.05 & 0.48 & 0.1 & 0.45 & -0.03 & 0.46 & -0.04 \\
0.4 & 0.05 & 0.43 & 0.11 & 0.5 & -0.02 & 0.52 & -0.03 \\
0.36 & 0.05 & 0.37 & 0.11 & 0.59 & -0.02 & 0.63 & -0.03 \\
0.31 & 0.05 & 0.31 & 0.11 & 0.62 & -0.01 & 0.66 & -0.03 \\
0.26 & 0.04 & 0.25 & 0.1 & 0.64 & -0.01 & 0.69 & -0.03 \\
0.21 & 0.04 & 0.2 & 0.09 & 0.69 & -0.01 & 0.75 & -0.03 \\
0.19 & 0.04 & 0.17 & 0.08 & 0.74 & 0 & 0.81 & -0.04 \\
0.16 & 0.04 & 0.14 & 0.07 & 0.76 & 0 & 0.83 & -0.04 \\
0.14 & 0.03 & 0.12 & 0.06 & 0.81 & 0 & 0.89 & -0.05 \\
0.12 & 0.03 & 0.09 & 0.05 & 0.86 & 0 & 0.95 & -0.05 \\
0.09 & 0.03 & 0.06 & 0.03 & 0.9 & 0 & 1.01 & -0.06 \\
0.07 & 0.02 & 0.04 & 0.02 & 0.95 & 0 & 1.06 & -0.07 \\
0.05 & 0.02 & 0.02 & 0 & 1 & 0 & 1.12 & -0.09 \\
\hline
\end{tabular}

Table 12: The mechanism's revolute joints for the morphing wing example in the configuration shown in Fig. 60.

\begin{tabular}{|c|cc|c|}
\hline \multirow{2}{*}{ Label } & \multicolumn{2}{|c|}{ R location } & Direction \\
\cline { 2 - 3 } & $\mathrm{X}$ & $\mathrm{Y}$ & of slide \\
\hline $\mathrm{a}$ & 1.00 & -0.06 & $156.80^{\circ}$ \\
\hline $\mathrm{b}$ & 0.96 & -0.05 & $153.43^{\circ}$ \\
\hline
\end{tabular}




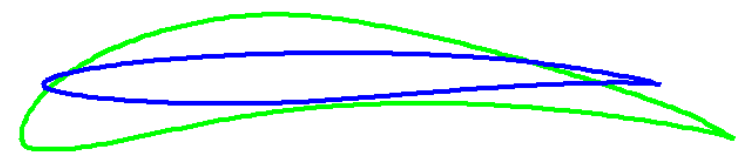

(a)

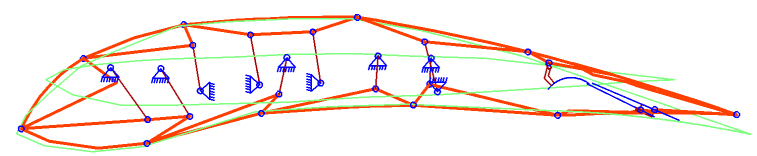

(c)

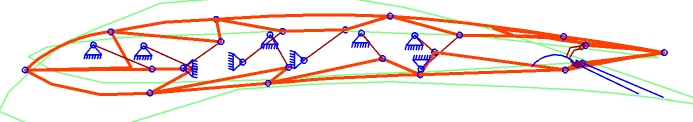

(e)

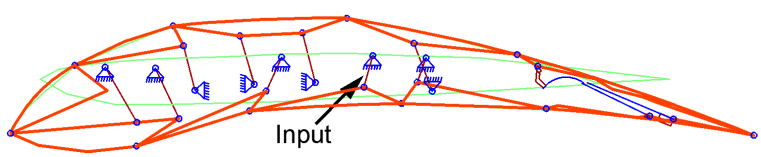

(b)

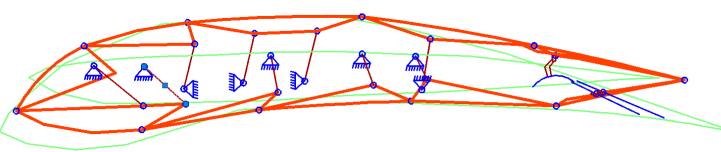

(d)

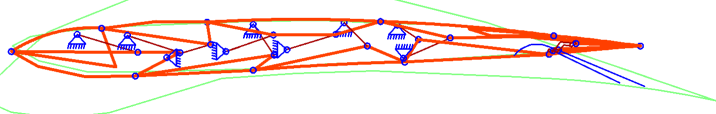

(f)

Figure 59: (a) Thin E850 high-speed airfoil and thick E420 high-lift airfoil. (b)-(f) Progression of the morphing wing between E420 and E850 airfoils. The two straight blue lines at the tail are paths for two sliders that guide the $\mathscr{C}$-segment. The curved sliders in red follow a curved path as it guides the other end of the $\mathscr{C}$-segment.

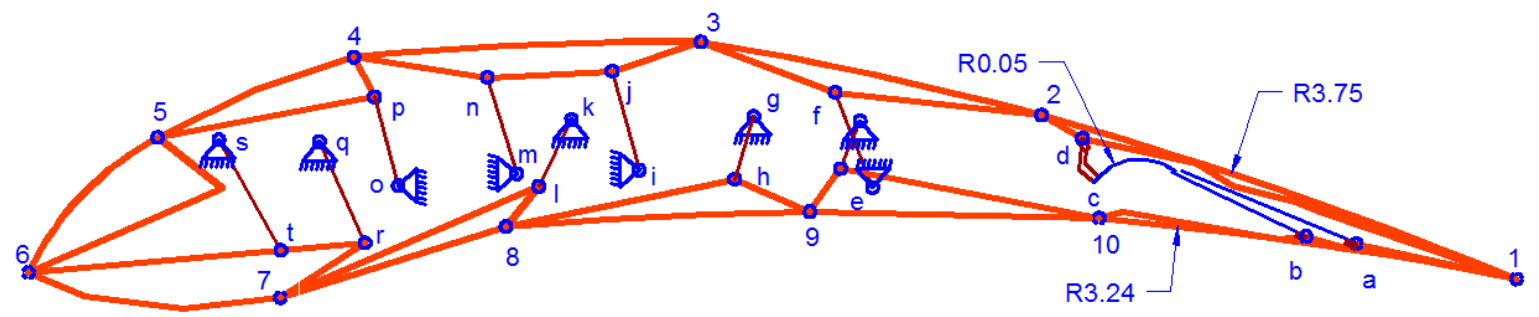

Figure 60: Labels for the dyads and revolute joints for the morphing airfoil example. 
Table 13: Mechanism points for the morphing airfoil example in the configuration shown in Fig. 60.

\begin{tabular}{|c|cc|}
\hline \multirow{2}{*}{ Joint } & \multicolumn{2}{|c|}{ R location } \\
\cline { 2 - 3 } & $\mathrm{X}$ & $\mathrm{Y}$ \\
\hline $\mathrm{c}$ & 0.80 & -0.01 \\
\hline $\mathrm{d}$ & 0.79 & 0.02 \\
\hline $\mathrm{e}$ & 0.62 & -0.01 \\
\hline $\mathrm{f}$ & 0.59 & 0.06 \\
\hline $\mathrm{g}$ & 0.52 & 0.04 \\
\hline $\mathrm{h}$ & 0.51 & -0.01 \\
\hline $\mathrm{i}$ & 0.43 & 0.00 \\
\hline $\mathrm{j}$ & 0.41 & 0.08 \\
\hline $\mathrm{k}$ & 0.38 & 0.04 \\
\hline $\mathrm{l}$ & 0.35 & -0.01 \\
\hline $\mathrm{m}$ & 0.33 & 0.00 \\
\hline $\mathrm{n}$ & 0.31 & 0.07 \\
\hline $\mathrm{o}$ & 0.24 & -0.01 \\
\hline $\mathrm{p}$ & 0.22 & 0.06 \\
\hline $\mathrm{q}$ & 0.18 & 0.02 \\
\hline $\mathrm{r}$ & 0.21 & -0.05 \\
\hline $\mathrm{s}$ & 0.10 & 0.02 \\
\hline $\mathrm{t}$ & 0.15 & -0.06 \\
\hline
\end{tabular}

Table 14: Revolute joints for the morphing airfoil example in the configuration shown in Fig. 60.

\begin{tabular}{|c|cc|}
\hline \multirow{2}{*}{ Joint } & \multicolumn{2}{|c|}{ R location } \\
\cline { 2 - 3 } & $\mathrm{X}$ & $\mathrm{Y}$ \\
\hline 1 & 1.13 & -0.09 \\
\hline 2 & 0.75 & 0.04 \\
\hline 3 & 0.48 & 0.10 \\
\hline 4 & 0.21 & 0.09 \\
\hline 5 & 0.05 & 0.03 \\
\hline 6 & -0.05 & -0.08 \\
\hline 7 & 0.15 & -0.10 \\
\hline 8 & 0.33 & -0.05 \\
\hline 9 & 0.57 & -0.03 \\
\hline 10 & 0.08 & -0.04 \\
\hline
\end{tabular}


circle, tear drop, and ellipse shapes used in the example. The arc lengths of the curves are similar. Data points for this study are shown in Table 15.

Figure $61 \mathrm{~b}$ shows the same shapes with different arc lengths, $C_{1}=25.12, C_{2}=26.36$, and $C_{3}=28.15$ units respectively. New target profiles are created by specifying the desired piece length of $s_{d}=0.1$. The resulting average piece length across the target profiles is $\bar{s}=0.10004$.

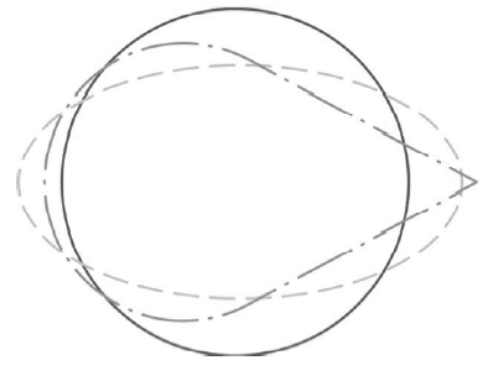

(a)

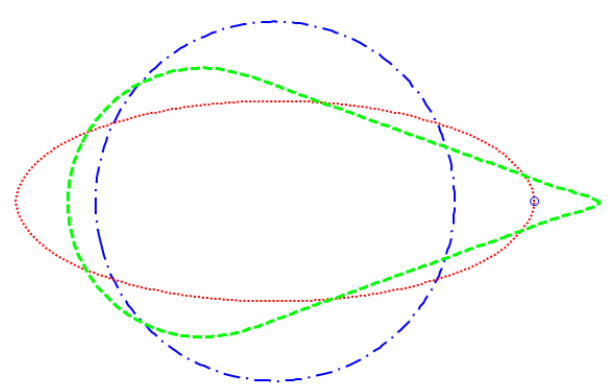

(b)

Figure 61: (a) The three profiles of the original flow field example. (b) The new profiles have significant differences in arc lengths.

This example uses six segments with two $\mathscr{C}$-segments, thus increasing the number of joints in the chain. Figures $62 \mathrm{a}$ and $62 \mathrm{~b}$ show the results from the segmentation process. Being symmetrical, the $[\mathscr{M} \mathscr{C} \mathscr{M} \mathscr{M} \mathscr{C} \mathscr{M}]$ design is selected as it simplifies the mechanism synthesis, noting that this design does not have the least maximum error. It has an $E_{\text {max }}=0.31$, whereas design $[\mathscr{M} \mathscr{M} \mathscr{M}$ $\mathscr{M} \mathscr{C} \mathscr{M}]$ has $E_{\max }=0.25$. However, the design selected has symmetry between the top and the bottom halves of the chain of rigid-bodies. Symmetry was sought in order to simplify the search for a working mechanism. The dyad connecting the bottom $\mathscr{C}$-segment to the frame is a binary link and it serves as the input. Persinger et al. [79] reported that for their eight-segment chain, the maximum 
Table 15: Points used in constructing the circle, ellipse, and tear drop profiles.

\begin{tabular}{|cc||cc||cc|}
\hline \multicolumn{2}{|c||}{ Circle } & \multicolumn{2}{c||}{ Ellipse } & \multicolumn{2}{c|}{ Tear Drop } \\
\hline $\mathrm{X}$ & $\mathrm{Y}$ & $\mathrm{X}$ & $\mathrm{Y}$ & $\mathrm{X}$ & $\mathrm{Y}$ \\
\hline 14.0 & 10.0 & 15.8 & 10.0 & 17.2 & 10.0 \\
13.9 & 10.8 & 15.3 & 10.7 & 16.4 & 10.3 \\
13.7 & 11.6 & 14.7 & 11.3 & 15.5 & 10.6 \\
13.3 & 12.3 & 14.0 & 11.6 & 14.7 & 10.9 \\
12.8 & 12.9 & 13.1 & 11.9 & 13.8 & 11.2 \\
12.1 & 13.4 & 12.3 & 12.0 & 13.0 & 11.5 \\
11.4 & 13.7 & 11.5 & 12.2 & 12.1 & 11.8 \\
10.6 & 13.9 & 10.6 & 12.2 & 11.3 & 12.1 \\
9.8 & 14.0 & 9.8 & 12.2 & 10.4 & 12.4 \\
9.0 & 13.9 & 9.0 & 12.2 & 9.6 & 12.7 \\
8.3 & 13.6 & 8.1 & 12.1 & 8.7 & 12.9 \\
7.6 & 13.2 & 7.3 & 12.0 & 7.8 & 12.9 \\
7.0 & 12.6 & 6.5 & 11.7 & 6.9 & 12.6 \\
6.5 & 11.9 & 5.7 & 11.4 & 6.2 & 12.0 \\
6.2 & 11.2 & 4.9 & 11.1 & 5.7 & 11.3 \\
6.0 & 10.4 & 4.5 & 10.4 & 5.5 & 10.4 \\
6.0 & 9.6 & 4.5 & 9.7 & 5.5 & 9.5 \\
6.2 & 8.8 & 4.9 & 8.9 & 5.7 & 8.7 \\
6.5 & 8.1 & 5.7 & 8.6 & 6.2 & 7.9 \\
7.0 & 7.4 & 6.5 & 8.3 & 6.9 & 7.4 \\
7.6 & 6.9 & 7.3 & 8.0 & 7.8 & 7.1 \\
8.3 & 6.4 & 8.1 & 7.9 & 8.7 & 7.0 \\
9.0 & 6.2 & 9.0 & 7.8 & 9.6 & 7.2 \\
9.8 & 6.0 & 9.8 & 7.8 & 10.4 & 7.5 \\
10.6 & 6.1 & 10.6 & 7.8 & 11.3 & 7.8 \\
11.4 & 6.3 & 11.5 & 7.9 & 12.1 & 8.1 \\
12.1 & 6.6 & 12.3 & 8.0 & 13.0 & 8.5 \\
12.8 & 7.1 & 13.1 & 8.2 & 13.8 & 8.8 \\
13.3 & 7.7 & 14.0 & 8.4 & 14.7 & 9.1 \\
13.7 & 8.4 & 14.7 & 8.8 & 15.5 & 9.4 \\
13.9 & 9.2 & 15.3 & 9.3 & 16.4 & 9.7 \\
14.0 & 10.0 & 15.8 & 10.0 & 17.2 & 10.0 \\
\hline & & & & & \\
\hline
\end{tabular}


error for all three profiles was 0.3 as opposed to 0.31 in this example. Note that the profiles used in the two experiments are dissimilar in arc lengths.

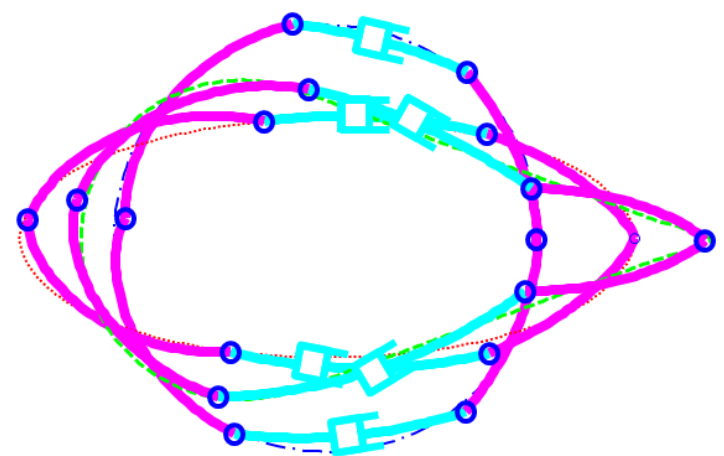

(a)

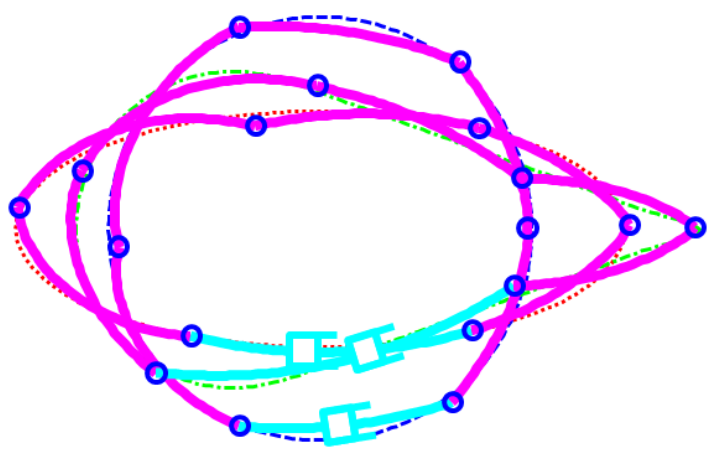

(b)

Figure 62: The chains of six segments are shown here in the two designs, with (a) imposing symmetry between segments in top half of the chain and those in the bottom half.

The synthesis goal was to achieve a single-DOF system. Figures 63a through 63e illustrate the shape change from an ellipse to tear drop and then to a circle. Note the monotonic input as the short crank labeled in Fig. 63a. One of the objectives of this task is to place all the additional links that move the chain within the closed loop. As concluded by Persinger et al. [79], the segmentation accuracy is likely to improve as the number of segments (or joints) increases.

In a flow-field control mechanism, the sliding path that protrudes at the back may disrupt the flow. Hence, some possible solutions are displayed in Fig. 64 that include the use of a slot embedded in the floor or frame, and a telescoping device that extends from inside. The detailed design of the mechanisms is defined by the pivot points shown in Fig. 65 and Tables 16 and 17. 


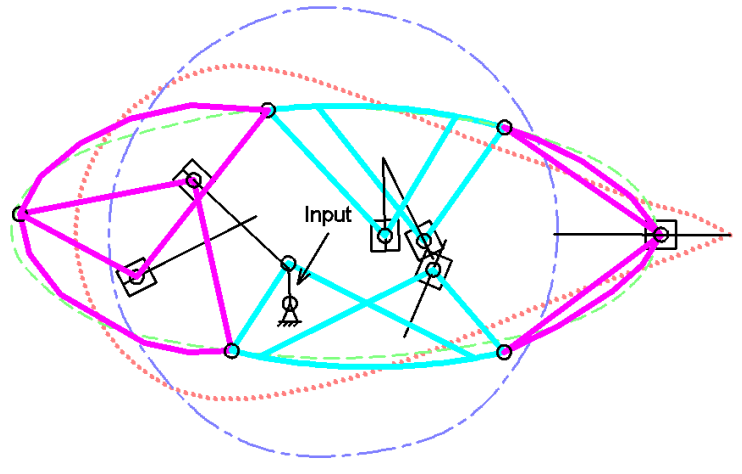

(a)

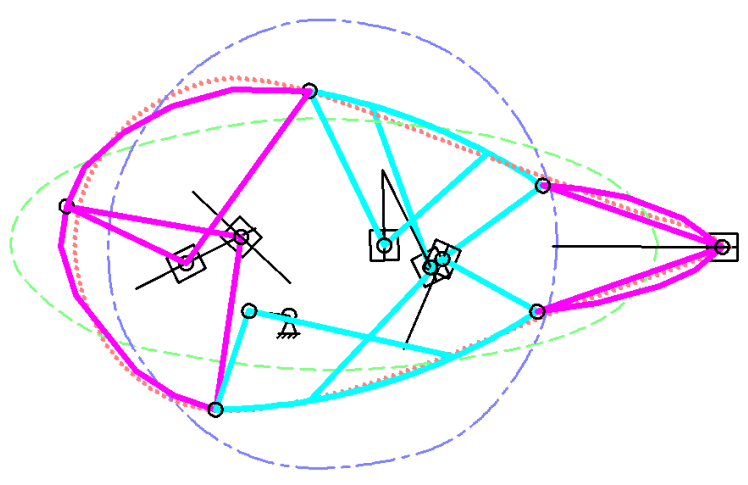

(c)

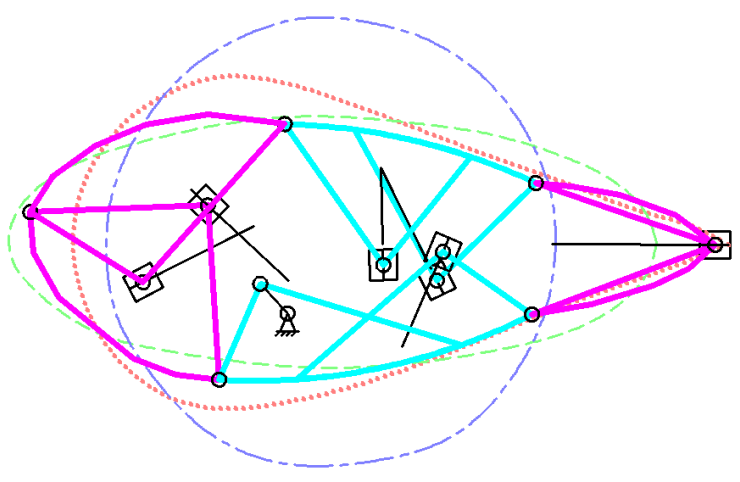

(b)

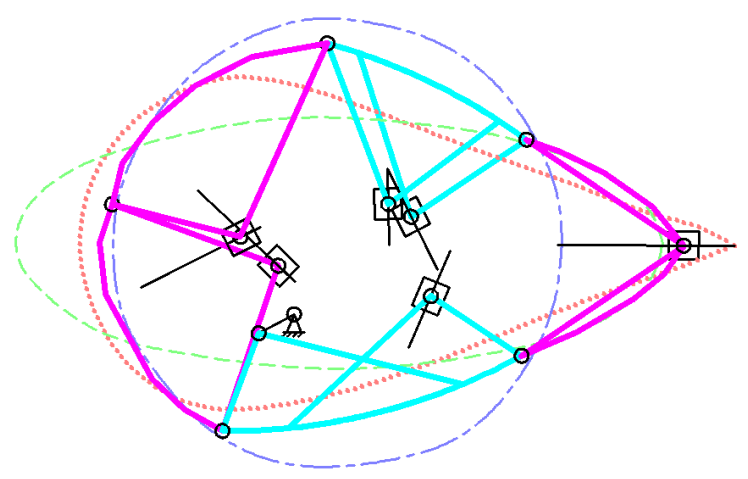

(d)

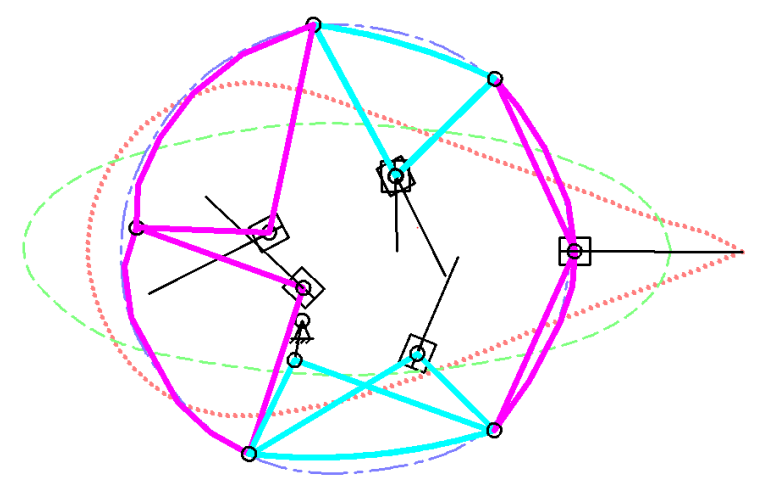

(e)

Figure 63: (a) The mechanism aligned with the ellipse. (b) An interim position between the ellipse and the tear-drop. (c) The mechanism aligned with the tear-drop. (d) An interim position between the tear-drop and the circle. (e) The mechanism aligned with the circle. 


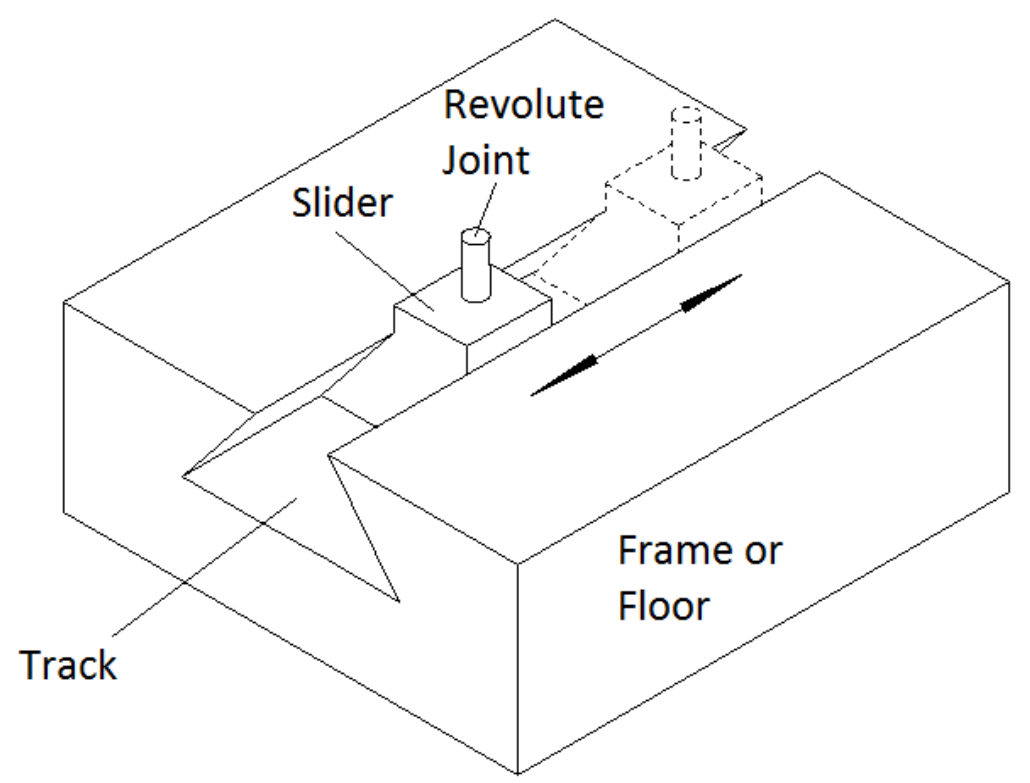

(a)

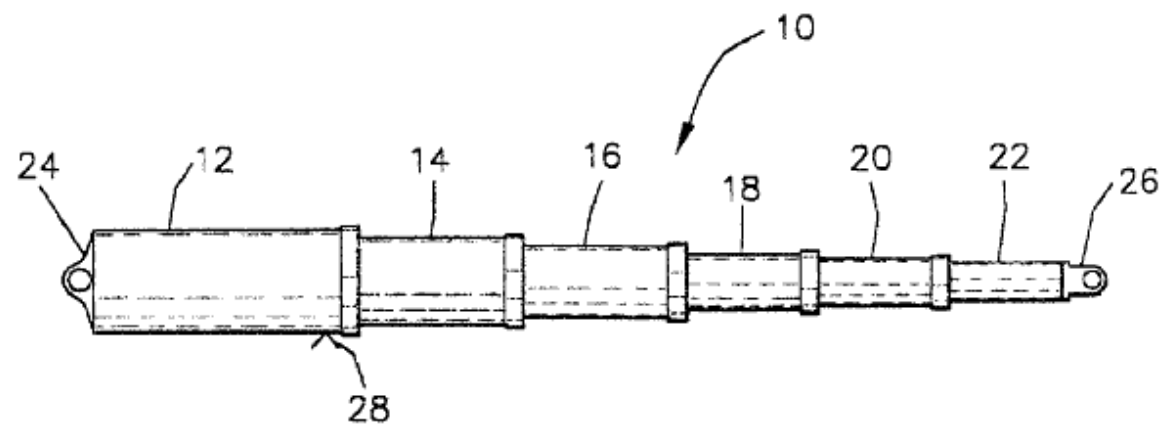

(b)

Figure 64: (a) A slider and a slot that can be entrenched in the floor or frame. (b) A telescoping element that can operate from inside the device for flow-field control [118]. . 


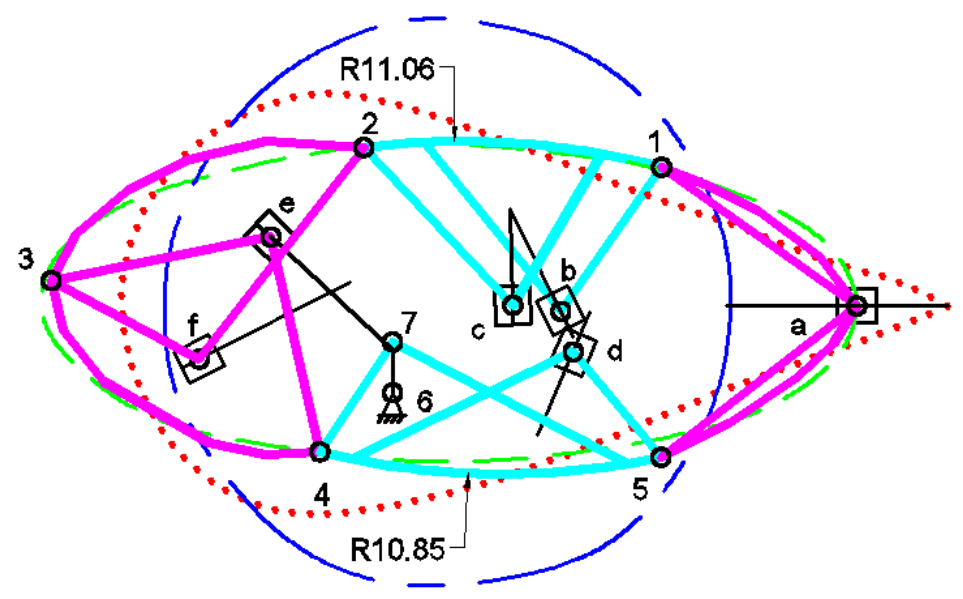

Figure 65: Labels for the dyads and revolute joints for the flow-field example.

Table 16: The mechanism's revolute joints for the flow-field example in the configuration shown in Fig. 65 .

\begin{tabular}{|c|cc|c|}
\hline \multirow{2}{*}{ Label } & \multicolumn{2}{|c|}{ R location } & Direction \\
\cline { 2 - 3 } & $\mathrm{X}$ & $\mathrm{Y}$ & of slide $^{\circ}$ \\
\hline $\mathrm{a}$ & 15.75 & 9.98 & $-0.24^{\circ}$ \\
\hline $\mathrm{b}$ & 11.57 & 9.91 & $-63.62^{\circ}$ \\
\hline $\mathrm{c}$ & 10.89 & 9.99 & $-88.51^{\circ}$ \\
\hline $\mathrm{d}$ & 11.74 & 9.33 & $67.00^{\circ}$ \\
\hline $\mathrm{e}$ & 7.47 & 10.96 & $-42.86^{\circ}$ \\
\hline $\mathrm{f}$ & 6.46 & 9.23 & $27.01^{\circ}$ \\
\hline
\end{tabular}

Table 17: Segment and linkage joints for the flow-field example in the configuration shown in Fig. 65.

\begin{tabular}{|c|cc|}
\hline \multirow{2}{*}{ Joint } & \multicolumn{2}{|c|}{ R location } \\
\cline { 2 - 3 } & $\mathrm{X}$ & $\mathrm{Y}$ \\
\hline 1 & 12.99 & 11.94 \\
\hline 2 & 8.78 & 12.22 \\
\hline 3 & 4.36 & 10.34 \\
\hline 4 & 8.16 & 7.92 \\
\hline 5 & 12.98 & 7.85 \\
\hline 6 & 9.19 & 8.76 \\
\hline 7 & 9.19 & 9.47 \\
\hline
\end{tabular}




\section{CHAPTER VI}

\section{CONCLUSIONS AND FUTURE WORK}

\subsection{Contributions}

This dissertation builds upon the developing theory of rigid-body shape-changing mechanism design. This area has already yielded many intriguing design concepts, even with the limitation of roughly equal arc lengths between design profiles as has been the standard prior to this work. The extension in this work, to design profiles that vary significantly in their arc lengths, is expected to address many new design possibilities. Some of these possibilities were highlighted in Chapter V.

A novel method for generating target profiles was established in which piecewise linear curves are used to solve these shape-changing design challenges. The target profile generation is performed such that points representing the design profiles are approximately the same distance apart across all profiles. As such, longer profiles contain more points. The advantage is that the same number of points taken along multiple profiles represents the same arc length along those profiles. This is ideal for approximation by a single rigid body. Moreover, the curvatures may be readily identified along the profiles, and, where similar both along a profile and between profiles, they define regions of ideal approximation by segments containing prismatic joints. 
Part of identifying rigid bodies along profiles requires comparing ordered sets of points. This problem is familiar to the image registration community, where obtaining the parameters in a similarity transformation that minimizes the sum of the squares of the distances between points on a reference image and the corresponding points in a sensed image frequently arises. In the design of planar shape-changing mechanisms, the point sets are confined to two dimensions. Restricting the problem in this way, a closed-form solution to the similarity transformation problem was developed. Even though the scaling parameter in the similarity transformation can be determined as part of the new derivation, the rigid-body criterion requires that we consider the problem as having no scaling. In the future, the chain can be made of flexible elements that can make scale changes and benefit from this derivation. Meanwhile, the current design uses these similarity transformations frequently when aligning data sets to create an average version of these sets, and then in aligning this average set with the original data.

The curvature at any point on a target profile is readily calculated using the point prior and the point following. Due to the discrete nature of a piecewise linear profile, the curvatures along the profile are not necessarily smooth. The effects of using a smoothed curvature and an average piece length to generate new target profiles were considered. Although these profiles have definite advantages, examples show a slight loss in accuracy versus the original design profiles. Also, techniques were generated that produced reliable results without the requirement of these target profiles.

A new segmentation process was formulated for cases where the arc lengths of the design profiles vary significantly. There are two basic types of rigid-body segments, mean segments or $\mathscr{M}$ segments and constant curvature segments or $\mathscr{C}$-segments. $\mathscr{M}$-segments are formed from the geometric centers of corresponding points from the same sections on the set of target profiles, where these sections are aligned at their error minimizing positions relative to each other. $\mathscr{C}$-segments 
share the same radius of curvature and include a prismatic joint to allow for extension in length. After identifying a design vector to indicate the number of segments in a profile-approximating chain and the ordering of $\mathscr{M}$ - and $\mathscr{C}$-segments, a segment matrix is generated. The segment matrix allows for the effective handling of optimizing the chain to minimize the error of the $\mathscr{M}$ - and $\mathscr{C}$-segments relative to the target profiles. Many initial segment matrices are generated to allow for the possibility of local minima in the error minimization process. This is the algorithm developed in Chapter III and implemented in Chapter V. Moreover, the process was implemented to cycle through all design vectors of the same length, typically generating many competing designs for the same set of profiles. After finding the error minimizing set of segments, they are connected to form a chain of rigid-bodies that would approximate all the design profiles. An optimization matched the segments' ends and aligned them with the profiles. In the cases of fixed-end and closed profiles, the additional constraints were also imposed on the optimization.

The mechanical complexity of a chain of rigid-bodies is reduced by fewer joints. To this end, compound segment types were considered. A compound segment is a merging of $\mathscr{M}$ - and $\mathscr{C}$ segments or two $\mathscr{C}$-segments when the angles at the revolute joints between them did not vary significantly. Note that $\mathscr{M}$-segments are not joined due to the fact that a new design vector should be attempted where the two $\mathscr{M}$-segments are considered one. An example shows that compound segments can produce chains with errors lower than those composed of only $\mathscr{M}$ - and $\mathscr{C}$-segments for the same number of joints.

A high-level discussion of the "ShapeChanger" application developed within MATLAB is presented. The chapter details the general structure of the code and focuses on the interplay of coefficients that must be selected to enable profile generation and segmentation to work smoothly. The use of the segment matrix, developed in Chapter III, was critical in this process. In addition, Appendix A contains a step-by-step guide to running ShapeChanger. 
Segmentation and mechanization examples of all three profile types - open, closed, and fixedend - were generated. Open profile examples included the car seat and the " $U$ " to " $D$ " transformation. The car seat example revealed that it could accommodate the ergonomics of different sized drivers with a simple single-DOF mechanism. The "U" to " $D$ " example demonstrated the ability to transform between the two letters when their arc lengths are significantly different. The closed profile example was of a wing that transformed between E420 and E850 airfoils. This example displayed that shape-change was possible even when the constraints dictated all mechanism links to be inside the wing compartment. The second closed-profile example pertained to flow-field control to affect flow around an object by changing between circle, ellipse, and teardrop shapes. The system developed was single-DOF with all mechanism links inside the shape. The fixed-end profiles dealt with the rework of the wing slat mechanism to eliminate the gap and yet extend out as far as in the conventional system. The system proposed used two $\mathscr{C}$-segments to achieve the required extension.

\subsection{Future Work}

Some directions for future work in the area of rigid-body shape-change include the following.

The segmentation process can be made sensitive to the type of mechanization preferred. $\mathscr{C}$ segments in very different locations along the chain can produce similarly small errors, but the chain may have differing desirability dependent on the location. For example, selecting the location for the prismatic joint where it encounters the smallest side loading is likely to be beneficial. Thus, given more information about operating conditions, designs could be weighted based on mechanical utility.

Closed profiles do not have definite end points for the segments. Currently, the assumption is made that closed profiles have end points, and an $\mathrm{R}$ joint is guaranteed to be there. This is a false constraint in that this point should be allowed to drift to generate the segmentation most 
advantageous to error reduction. The problem with letting the end points move without design guidance is that this may yield a chain that needs to "flip" between aligning with the design profiles, resulting in an unwieldy mechanization challenge. In this work, designers are granted the liberty to change the starting point to locations that are advantageous in segmentation and mechanization processes. Nonetheless, a more sophisticated method could determine start and end points on each closed profile. Figure 66 illustrates the problems related to starting and ending points for closed profiles and displays how open profiles could pose a similar challenge.

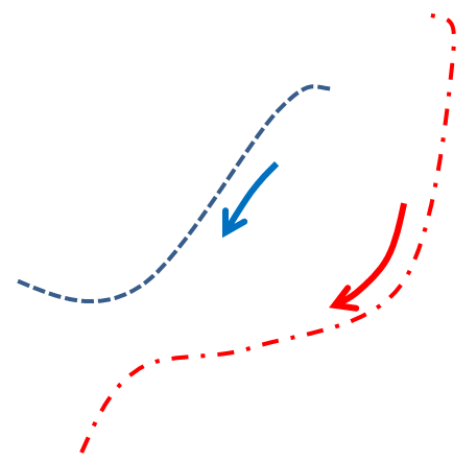

(a)

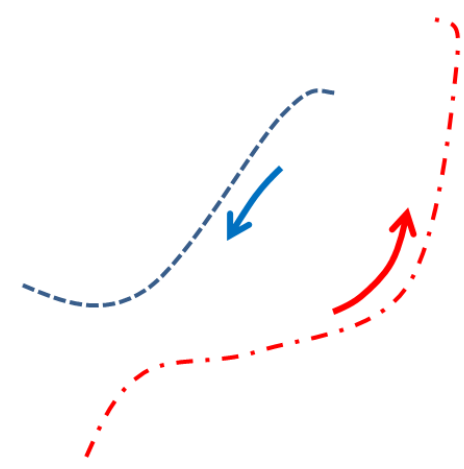

(b)

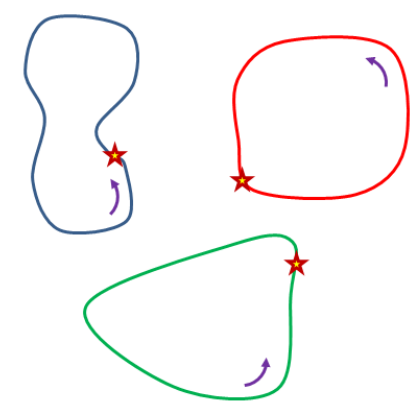

(c)

Figure 66: (a) The mechanization challenges posed by (a) and (b) are significantly different. Associating the identified point as being at the same location in the segmentation in (c) may be an accurate way of approximating bodies, but poses a significant mechanization challenge. 
The relative placement of design profiles needs further consideration. Although this does not affect segmentation, it significantly affects mechanization. The reason this is identified as a challenge here is that the curve placement and generation is viewed as part of the procedures presented in this dissertation. Identifying the relative positions of design profiles, when they are freely specified, such that it is most advantageous to the mechanization is an open question.

Relative curve sizes in the set of design profiles can also be studied. Recall the wing design challenge in Chapter V, where the high-lift profile was desired to be longer relative to the gliding profile. The design challenge specified that a longer lower camber was better. Even though the current method can address any desired change in arc length to solve the problem, the desirable number of prismatic joints or telescopic links is likely to be very small due to practical considerations. Moreover, the amount of expansion of each $\mathscr{C}$-segment should enter as a design variable, unaddressed in this work.

The methodologies developed could be expanded to spatial shape-changing mechanisms. Readily envisioned is a set of spatial bodies connected by universal (U) or spherical (S) joints allowing matching of space curves. Viewing $\mathrm{U}$ or $\mathrm{S}$ joints as spatial equivalents of the revolute joint, there is no parallel to the $\mathrm{P}$ joint. A cylindrical joint, to allow sliding and rotation, requires a straight line of slide to be mechanically feasible. Thus, to keep P joints in the spatial chain requires sections of the curves to be both roughly constant curvature and roughly planar. A helical connection may be posed as telescoping, but the practicalities of such a connection raise serious concerns. These length constraints may pose a serious challenge to approximating space curves of different arc lengths. Then, given an acceptable segmentation, mechanization introduces a host of beguiling challenges.

A surface can be constructed via a set of planar cross-sections as in the designs of modern ships and aircraft wings. Each planar cross-section will include a planar curve representing some local portion of the surface. This curve can be generated via the techniques presented in this dissertation. 
Chains of rigid-body segments on separate parallel planes could be joined together to form a shapechanging surface. This series of segmentations needs to be studied to see if it could produce a meaningful mechanical design.

Although the curvature plot work in Chapter III was not developed further, it could prove to create better initial segment matrices. The knowledge of the ideal locations for a $\mathscr{C}$-segment is contained in a comparison of the curvature plots, but performing this comparison efficiently does not yield to a simple or reasonably quick algorithm.

Finally, at the boundary between compliance and rigid-body devices, there is the possibility of implementing a new segmentation process. The pseudo rigid-body compliant mechanism model can begin with exactly the results established in this work. Instead of revolute joints identified at a specific point, living hinges may be represented by a collection of successive points. Figure 67 shows the result of preliminary work on this concept where all the profiles have the same arc length and the living hinges have six pieces allocated for each of them. The segments are treated as $\mathscr{M}$-segments, and the living hinges are any collection of pieces between them. 


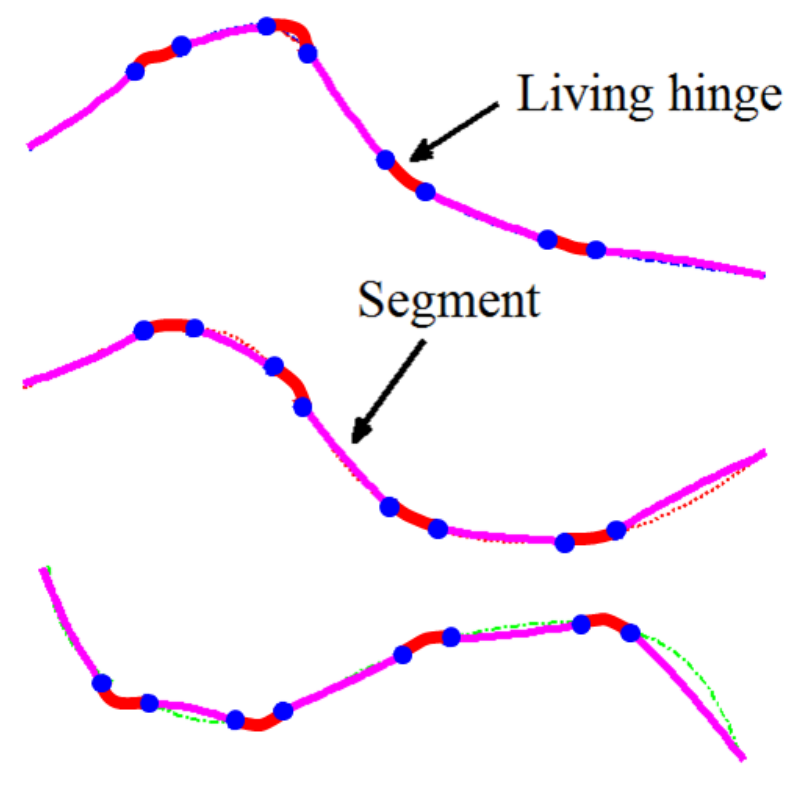

Figure 67: Initial results for a compliant mechanism with living hinges, approximating three profiles. 


\section{BIBLIOGRAPHY}

[1] Sofla, A. Y. N., Meguid, S. A., Tan, K. T., and Yeo, W. K., 2010, "Shape Morphing of Aircraft Wing: Status and Challenges," Material Design, 31(3), pp. 1284-1292.

[2] Vasista, S., Tong, L., and Wong, K.C., 2012, "Realization of Morphing Wings: A Multidisciplinary Challenge," Journal of Aircraft, 49(1), pp. 11-28.

[3] Frank, G. J., Joo, J. J., Sanders, B. P., Garner, D. M., and Murray, A. P., 2008, "Mechanization of a High Aspect Ratio Wing for Aerodynamic Control," Journal of Intelligent Material Systems and Structures, 19(9), pp. 1101-1112.

[4] Inoyama, D., Sanders, B. P., and Joo, J. J., 2008, "Topology Optimization Approach for the Determination of the Multiple-Configuration Morphing Wing Structure," Journal of Aircraft, 45(6), pp. 1853-1862.

[5] Kota, S., Hetrick,, J., and Osborn, R., 2003, "Design and Application of Compliant Mechanisms for Morphing Aircraft Structures," Proc. of the SPIE, 5054, pp. 24-33.

[6] Anderson, J. D., 2012, Fundamentals of Aerodynamics, McGraw-Hill, New York, NY.

[7] Abdulrahim, M., Garcia, H., and Lind, R., 2005, "Flight Characteristics of Shaping the Membrane Wing of a Micro Air Vehicle," Journal of Aircraft, 42(1), pp. 131 - 137.

[8] Limaye, P., Ramu, G., Pamulapati, S., and Anathasuresh, G. K., 2012, "A Compliant Mechanism Kit with Flexible Beams and Connectors Along with Analysis and Optimal Synthesis Procedures," Mechanism and Machine Theory, 49(3), pp. 21-39.

[9] Strelec, J. K. and Lagoudas, D. C., 2002, "Fabrication and Testing of a Shape Memory Alloy Actuated Reconfigurable Wing," SPIE Smart Structures and Materials: Smart Structures and Integrated Systems, 4701, pp. 267-280.

[10] Weisshaar, T. A., 2006, "Morphing Aircraft Technology - New Shapes for Aircraft Design," Proceedings of the Multi functional Structures Integration of Sensors and Antennas Meeting, RTO-MP-AVT-141, Neuilly-sur-Seine, France, pp. 01:1-20. 
[11] Prock, B. C., Weisshar, T. A., and Crossley, W. A., 2002, "Morphing Airfoil Shape Change Optimization with Minimum Actuator Energy as an Objective," Proceedings of the $9^{\text {th }}$ AIAA/ISSMO Symposium on Multidisciplinary Analysis and Optimization, September 4-6, Atlanta, GA, U.S.A., AIAA 2002-5401.

[12] Kota, S., Osborn, R., Ervin, G., Maric, D., Flick, P., and Paul, D., 2009, "Mission Adaptive Compliant Wing - Design, Fabrication and Flight Test," Proceedings of the RTO Applied Vehicle Technology Panel (AVT) Symposium, RTO-MP-AVT-168, Evora, Portugal, 20-24 April.

[13] Santer, M. J. and Pelligrino, S., 2007, "Topology Optimization of Adaptive Compliant Aircraft Wing Leading Edge," Proceedings of the $48^{\text {th }}$ AIAA/ASME/ASCE/AHS/ASC Structures, Structural Dynamics, and Materials Conference, Honolulu, Hawaii, U.S.A., 23-24 April.

[14] Erdman, A., Sandor, G., and Kota, S., 2001, Mechanism Design: Analysis and Synthesis, 3rd ed., vol. 1, Prentice Hall, Inc., Englewood Cliffs, NJ.

[15] Pettey, B., 2003, “Adjustable Automotive Airfoil,” U.S. Patent US6,540,282B2.

[16] Cesnik, C. E. S. and Shin, S., 2001, "On the Modeling of Integrally Actuated Helicopter Blades," International Journal of Solids and Structures, 38(10), pp. 1765 - 1789.

[17] Wong, J. T., 2007, "Shape Morphing Structures via Intercalation Compounds," M.Eng. Thesis, Massachusetts Institute of Technology.

[18] Grohmann, B., Maucher, C., and Janker, P., 2006, "Actuation Concepts for Morphing Helicopter Rotor Blade," Proceedings of the $25^{\text {th }}$ International Congress of the Aeronautical Sciences - ICAS2006, Silicon Valley, CA, U.S.A., 23-24 April.

[19] Warwick, G., 2010, "Sikorsky Spins Up Adaptive Rotor Work," Aviation Week, February 22, from http://www.aviationweek.com/.

[20] Barlas, T. K., and van Kuik, G. A. M., 2010, "Review of State of the Art in Smart Rotor Control Research for Wind Turbines," Progress in Aerospace Sciences, 46(1), pp. 1-27.

[21] Gaunaa, M., 2010, "Unsteady Two-Dimensional Potential-Flow Model for Thin Variable Geometry Airfoils," Wind Energy, 13(2-3), pp. 167-192.

[22] Lambie, B., 2011, "Aeroelastic Investigation of a Wind Turbine Airfoil with Self-Adaptive Camber," Ph.D. Dissertation, Technische Universitat Darmstadt, Denmark.

[23] Maheri, A., Noroozi, S., and Vinney, J., 2007, "Application of Combined Analytical/FEA Coupled Aero-Structure Simulation in Design of Wind Turbine Adaptive Blades," Renewable Energy, 32(12), pp. 2011-2018.

[24] Pechlivanoglou, G., Nayeri, C. N., and Paschereit, C. O., 2012, "Performance Optimization of Wind Turbine Rotors with Active Flow Control - Part 2," Mechanical Engineering, 134(8), p. 55. 
[25] Kota, S. and Hetrick, J. A., 2008, “Adaptive Compliant Wing and Rotor System,” U.S. Patent US7,384,016B2.

[26] Gillmer, T. C., 1975, Modern Ship Design, 2nd ed., Naval Institute Press, Annapolis, MD.

[27] Molland, A. F., 2008, The Maritime Engineering Handbook: A Guide to Ship Design, Construction and Operation, Butterworth-Heinemann, Burlington, MA.

[28] BAE Systems, 2012, "The Astute Launch: Tour the Submarine," from http://production.investis.com/astute/about/tour/.

[29] Garner, L. J., Wilson, L. N., Lagoudas, D. C., and Rediniotis, O. K., 2000, "Development of a Shape Memory Alloy Actuated Biomimetic Vehicle," Smart Materials and Structures, 9(5), pp. 673-683.

[30] Gernez, E., Harada, C. M., Bootsman, R., Chaczko, Z., Levine, G., and Keen, P., 2012, "Protei Open Source Sailing Drones: A Platform for Education in Ocean Exploration and Conservation," Proceedings of the 2012 International Conference on Information Technology Based Higher Education and Training, ITHET 2012, Istanbul, Turkey, June 21 - 23, DOI: 10.1109/ITHET.2012.6246036.

[31] Washington, G. N., 1996, "Smart Aperture Antennas," Smart Materials and Structures, 5(6), pp. 801-805.

[32] Martin, J. W., Main, J. A., and Nelson, G. C., 1998, "Shape Control of Deployable Membrane Mirrors," ASME Adaptive Structures and Materials Systems Conference, Aneheim, CA.

[33] Lu, K. J. and Kota, S., 2003, "Design of Compliant Mechanisms for Morphing Structural Shapes," Journal of Intelligent Material Systems and Structures, 14(3), pp. 379-391.

[34] Lu, K. J., 2004, "Synthesis of Shape Morphing Compliant Mechanisms," Ph.D. Dissertation, The University of Michigan.

[35] Plecnik, M., 2010, "Design of A Shape-Changing Rigid-Body Parabolic Light Reflector," Honors Thesis, The University of Dayton.

[36] Gupta, V. K., 2011, "Modified Approach for Optimum Position and Sizing of Piezoelectric Actuator for Steering of Parabolic Antenna,” Tech Science Press, 6(2), pp. 65-75.

[37] Maji, A. K., Wegner, P., and Rochin, R., 2004, "Surface Actuation of Lightweight Mirrors with Shape Memory Alloy," Technical Notes of the Journal of Aerospace Engineering, 17(2), pp. 83-89.

[38] Stamper, B. L., Angel, J. R. P., Burge, J. H., and Woolf, N. J., 2000, "Flat Membrane Mirrors for Space Telescopes," Proceedings of SPIE: Imaging Technology and Telescopes, 4091, San Diego, CA, June 30, pp. $126-136$. 
[39] Fernández, E. and Artal, P., 2003, "Membrane Deformable Mirror for Adaptive Optics: Performance Limits in Visual Optics," Optic Express, 11(9), pp. 1056-1069.

[40] Heselhaus, U. and Richter, W., 2001, "Folding Roof for A Convertible," U.S. Patent $6,270,143 \mathrm{~B} 1$.

[41] Rau, D., Blume, H., and Boers, A., 1992, "Portable Performance Platform," U.S. Patent $5,078,442$.

[42] Mohd Zubir, B., Shirinzadeh, M. N. and Tian, Y., 2009, "A New Design of Piezoelectric Driven Compliant-Based Microgripper for Micromanipulation," Mechanism and Machine Theory, 44(12), pp. 2248-2264.

[43] Hirose, S., Umetani, Y., 1978, "The Development of Soft Gripper for the Versatile Robot Hand," Mechanism and Machine Theory, 13(3), pp. 351-359.

[44] Moon, Y. M., 2007, "Bio-Mimetic Design of Finger Mechanism with Contact Aided Compliant Mechanism," Mechanism and Machine Theory, 42(5),pp. 600-611.

[45] Petković, D. and Pavlović, N., 2012, "A New Principle of Adaptive Compliant Gripper," Springer: Mechanisms and Machine Science 3, 1(1), pp. 143-150.

[46] Murray, A. P., Schmiedeler, J. P., and Korte, B. M., 2008, "Kinematic Synthesis of Planar, Shape-Changing Rigid-Body Mechanisms," Journal of Mechanical Design, 130(3), pp. 1 10.

[47] Howell, L. L., 2001, Compliant Mechanisms, John Wiley \& Sons, New York, NY.

[48] Shuib, S., Ridzwan, M. I. Z., and Kadarman, A. H., 2007, “ Methodology of Compliant Mechanisms and Its Current Developments in Applications: A Review," American Journal of Applied Sciences, 4(3), pp. 160-167.

[49] Shigley, J. E. and Mischke, C. R., 1989, Mechanical Engineering Design, McGraw-Hill, New York, NY.

[50] Stoll, H. W., 1999, Product Design Methods and Practices, Marcel Dekker, New York, NY.

[51] Sen, D., Chowdhury, S., and Pandey, S. R., 2004, "Geometric Design of Interference-Free Planar Linkages," Mechanism and Machine Theory, 39(7), pp. 737-759.

[52] Ling, Z. K., and Chase, T. R., 1996, “A Priori Geometric Design of an Interference Free Complex Planar Mechanism,” Mechanism and Machine Theory, 31(4), pp. 513-524.

[53] Waldron, K. and Kinzel, G., 1999, Kinematics, Dynamics, and Design of Machinery, 2nd ed., John Wiley and Sons, Inc., New York, NY.

[54] Kota, S. and Erdman, A. G., 1997, "Motion Control in Product Design," Mechanical Engineering, 119(8), pp. 74-77. 
[55] Zotzmann, M. B. J. and Lendlein, A., 2010, "Shape-Memory Polymers and Shape-Changing Polymers," Advanced Polymer Science, 226, Springer-Verlag, Berlin.

[56] Jalili, N., Wagner, J., and Dadfarnia, M., 2003, "A Piezoelectric Driven Ratchet Actuator Mechanism with Application to Automotive Engine Valve," Mechatronics, 13(8-9), pp. 933956.

[57] Ramrkahyani, D. S., Lesieutre, G. A., Frecker, M., and Bharti, S., 2004, "Aircraft Structural Morphing using Tendon Actuated Compliant Cellular Trusses," In Proceeding of the $45^{\text {th }}$ AIAA/ASME/AHS/ASC Structures, Structural Dynamics \& Materials Conference, Palm Springs, CA, April 19-22, AIAA 2004-1728.

[58] Murphy, M. D., Midha, A., and Howell, L. L., 1996, "The topological synthesis of compliant mechanisms," Mechanism and Machine Theory, 31(2), pp. 185-199.

[59] Tanakorn, T. and Kota, S., 2007, "Design of Compliant Mechanisms for Minimizing Input Power in Dynamic Applications," Journal of Mechanical Design, 129(10), pp. 1064 - 1075.

[60] Tan, X., Kim, A., Usher, N., Laboy, D., Jackson, J., Kapetanovic, A., Rapai, J., Sabadus, B., and Zhou, X., 2006, "An Autonomous Robotic Fish for Mobile Sensing," Proceedings of the 2006 IEEE/RSJ International Conference on Intelligent Robots and Systems, Beijing, China, October 9 - 15.

[61] Speich, J. and Goldfarb, M., 2000, "A Compliant-Mechanism-Based Three Degree-ofFreedom Manipulator for Small-Scale Manipulation," Robotica, 18(1), pp. 95-104.

[62] Parlaktas, V., and Tanik, E., 2011, "Partially Compliant Slider-Crank (RSSP) Mechanism," Mechanism and Machine Theory, 46(11),pp. 1707-1718.

[63] Mankame, N. D. and Ananthasuresh, G. K., 2004, "A Novel Compliant Mechanism for Converting Reciprocating Translation into Enclosing Curved Paths," Journal of Mechanical Design, 126(7), pp. $667-672$.

[64] Trease, B. P., Moon, Y. M., and Kota, S., 2005, "Design of Large-Displacement Compliant Joint," Journal of Mechanical Design, 127(7), pp. 788-798.

[65] Hoagg, J., Bernstein, D., Lacy, S. L., and Venugopal, R., 2003, "Adaptive Control of a Flexible Membrane Using Acoustic Excitation," In Proceeding of the AIAA Guidance, Navigation, and Control Conference, Austin, TX, USA, August 11-14, AIAA 2003-5430.

[66] Otsuka, K. and Wayman, C. M., Eds., 1998, Shape Memory Materials, Cambridge University Press, Cambridge, U. K.

[67] Fan, K., Huang, W. M., Wang, C. C., Ding, Z., Zhao, Y., Purnawali, H., Liew, K. C., and Zheng, L. X., 2011, "Water-Responsive Shape Memory Hybrid: Design, Concept and Demonstration," eXPRESS Polymer Letters, 5(5), pp. 409-416. 
[68] Kim, J., Yun, S., and Ounaies, Z., 2006, "Discovery of Cellulose as a Smart Material," Macromolecules, 39(12), pp. 4202-4206.

[69] Reece, P. L., Ed., 2007, Progress in Smart Materials and Structures, Nova Science Publishers, New York, NY.

[70] Ko, F. K., and Yang, H., 2008, "Functional Nanofibre: Enabling Material for the Next Generations Smart Textiles," Journal of Fiber Bioengineering and Informatics, 1(2), pp. 81-92.

[71] Zanaboni, E., 2008, "One Way and Two Way-Shape Memory Effect: Thermo-Mechanical Characterization of Ni-Ti Wires,” Dissertation, Universitá Degli Studi Di Pavia.

[72] Stiles, L. A., Schaub, H., Maute, K. K., and Moorer, Jr., D. F., 2010, "Electrostatic Inflation of Membrane Space Structures," AAS/AIAA Astrodynamics Specialists Conference, August 2-5, AIAA 10-8134, Toronto, ON , Canada.

[73] Marquis, Z., 2008, "GINA Light Visionary Model: Shape-Changing Concept Coupe by BMW," Elite Choice, from http://elitechoice.org/2008/06/10/

[74] Huang, T., Liu, H. T., Chetwynd, D. G., 2011, "Generalized Jacobian Analysis of Lower Mobility Manipulators," Mechanism and Machine Theory, 46(6), pp. 831-844.

[75] Ding, H., Hou, F., and Kecskeméthy, A., and Huang, Z., 2012, "Synthesis of the Whole Family of Planar 1-DOF Kinematic Chains and Creation of Their Atlas Database," Mechanism and Machine Theory, 47(1), pp. 1-15.

[76] McCarthy, J. M. and Soh, G. S., 2010, Geometric Design of Linkages, 2nd ed., Springer, New York, NY.

[77] Balli, S.S. and Chand, S., 2002, "Defects in Link Mechanisms and Solution Rectification," Mechanism and Machine Theory, 37(9), pp. 851-876.

[78] Kinzel, E. C., Schmiedeler, J. P., and Pennock, G. R., 2006, "Kinematic Synthesis for Finitely Separated Positions Using Geometric Constraint Programming," Journal of Mechanical Design, 128(5), pp. 1070-1079.

[79] Persinger, J. A., Schmiedeler, J. P., and Murray, A. P., 2009, "Synthesis of Planar RigidBody Mechanisms Approximating Shape Changes Defined by Closed Curves," Journal of Mechanical Design, 131(7), pp. 1 - 7.

[80] Yao, J. and Angeles, J., 2000, "Computation of All Optimum Dyads in The Approximate Synthesis of Planar Linkages for Rigid-Body Guidance," Mechanism and Machine Theory, 35(8), pp. 1065-1078.

[81] Zhao, K., Schmiedeler, J. P., and Murray, A. P., 2012, "Design of Planar, Shape-Changing Rigid-Body Mechanisms for Morphing Aircraft Wings," Journal of Mechanisms and Robotics, 4(4), pp. 041007-1-10. 
[82] Perkins, D. A. and Murray, A. P., 2012, "Singularity Free Revolute-Prismatic-Revolute and Spherical-Prismatic-Spherical Chains for Articulating Planar and Spherical Single Degree of Freedom Mechanisms," Journal of Mechanisms and Robotics, 4(1), pp. 011007-1-6.

[83] Doshi, R., Yeh, C., and LeBlanc, M., 1998, "The Design and Development of a Gloveless Endoskeletal Prosthetic Hand," Journal of Rehabilitation Research and Development, 35(4), pp. 388-395.

[84] Winfrey, R. C., 2008, "Prosthetic Hand Having a Conformal, Compliant Grip and Opposable, Functional Thumb," U.S. Patent US7,361,197.

[85] Smith, A. M. O., 1975, "High-Lift Aerodynamics," Journal of Aircraft, 12(6), pp. 501-530.

[86] Kato, M., Sano, S., and Hiyoski, Y., 1996, "Variable Section Extrusion Die Set and Variable Extrusion Molding Method,” U.S. Patent 3,526,020.

[87] Choi, H. J., Lim, S. J., Shin, H. T., and Choi, S., 2008, "CNC Extruded for Varied Section Extrusion," Journal of Achievements in Materials and Manufacturing Engineering, 29(2), pp. $175-178$.

[88] O’Connor, J. E., 1991, "Method for Making Variable Cross Section Pultruded Thermoplastic Composite Article," U.S. Patent 5,026,447.

[89] Myszka, D. H., 2012, Professor at the University of Dayton, private communication.

[90] Zitová, B. and Flusser, J., 2003, "Image Registration Methods: A Survey,” Image and Vision Computing, 21(11), pp. $977-1000$.

[91] Horn, B. K. P., 1987, "Closed-Form Solution of Absolute Orientation Using Unit Quaternions," Journal of the Optical Society of America, 4(April), pp. 629-642.

[92] Horn, B. K. P., Milden, H. M., and Negahdaripour, S., 1988, "Closed-Form Solution of Absolute Orientation Using Orthonormal Matrices," Journal of the Optical Society of America, 5(July), pp. 1127-1135.

[93] Arun, K. S., Huang, T. S., and Blostein, S. D., 1987, "Closed-Form Solution of Absolute Orientation Using Orthonormal Matrices," Journal of the Optical Society of America, 9(5), pp. 698-700.

[94] Umeyama, S., 1991, "Least-Squares Estimation of Transformation Parameter Between Two Point Patterns," IEEE Transactions on Pattern Analysis and Machine Intelligence, 13(4), pp. 376-380.

[95] Wen, G., Wang, Z., and Xia, S., 1991, "Least-Squares Fitting of Multiple m-Dimensional Point Sets," Springer-Verlag: Visual Computer, 22, pp. 387-398.

[96] Golub, G. H. and Loan, C. F. V., 1983, Matrix Computation, John Hopkins University, Baltimore, MD. 
[97] Forsythe, G. E., Malcolm, M. A., and Moler, C. E., 1977, Computer Methods for Mathematical Computations, Prentice Hall, Englewood Cliffs, NJ.

[98] Sabuncu, M. R. and Ramadge, P., 2008, "Using Spanning Graphs for Efficient Image Registration," IEEE Transactions on Image Processing, 17(5), pp. 788-797.

[99] Burchard, H. G., 1994, "Discrete Curves and Curvature Constraints," Curves and Surfaces in Geometric Design, P.-J. Laurent, A. L. Méhauté, and L. L. Shumaker, Eds., A. K. Peters Ltd., Wellesly, MA.

[100] Stahl, S., 2005, Introduction to Topology and Geometry, John Wiley, Hoboken, NJ.

[101] Shikin, E. V., 1995, Handbook and Atlas of Curves, CRC Press, Boca Raton, FL.

[102] Casey, J., 1885, A Treatise on the Analytical Geometry of the Point, Line, Circle, and Conic Sections, Dublin University Press, Dublin, Ireland.

[103] Zill, D. G. and Cullen, M. R., 2006, Advanced Engineering Mathematics, 3rd ed., Jones \& Bartlett Learning, Sudbury, MA.

[104] Mokhtarian, F., and Mackworth, A. K., 1993, "Theory of Multi-Scale, Curvature-Based Shape Representation for Planar Curves," IEEE Transactions on Pattern Analysis and Machine Intelligence, 14(8), pp. 789-805.

[105] Nielson, G. M., 1993, "Scattered Data Modeling," IEEE Computer Graphics and Applications, 13(1), pp. 60-70.

[106] Eppler, R., 1990, Airfoil Design and Data, Springer-Verlag, New York, NY.

[107] Zhao, K., Schmiedeler, J. P., and Murray, A. P., 2011, "Kinematic Synthesis of Planar, ShapeChanging Rigid Body Mechanisms with Prismatic Joints," Proceedings of the ASME 2011 International Design Engineering Technical Conferences, Washington, D.C., August 28 - 31, DETC2011-48503.

[108] Shamsudin, S. A., Murray, A. P., Myszka, D. H., and Schmiedeler, J. P., 2011, "Kinematic Synthesis of Planar, Shape-Changing Rigid Body Mechanisms for Design Profiles with Significant Differences in Arc Length," Proceedings of the ASME 2011 International Design Engineering Technical Conferences, Washington, D.C., August 28 - 31, DETC2011-47657.

[109] Frey, J. K. and Tecklin, J. S., 1986, "Comparison of Lumbar Curves when Sitting on the Westnofa Balans ${ }^{\circledR}$ Multi-Chair, Sitting on a Conventional Chair, and Standing," Physical Therapy, 66, pp. 1365-1369.

[110] McCabe, P. T., 2004, Contemporary Ergonomics 2004, CRC Press, Boca Raton, FL.

[111] Pheasant, S., 1996, Bodyspace: Anthropometry, Ergonomics and the Design of Work, 2nd ed., Taylor \& Francis, London, U.K.. 
[112] Tilley, A. R., 2002, The Measure of Man and Woman, Revised ed., John Wiley \& Sons, New York, NY.

[113] Morrison, J. H., 1998, "Numerical Study of Turbulence Model Predictions for MD 30P30N and NHLP-2D Three-Element Highlift Configurations," NASA/CR-1998-208967.

[114] Mann, A., 2011, Slat Deployment Mechanism, U.S. Patent US0036944.

[115] Khorrami, M. R., 2003, "Understanding Slat Noise Sources," Colloquium EUROMECH 449, Chamonix, France, December 9 - 12.

[116] Abbott, I. H. and Von Doenhoff, A. E., 1959, Theory of Wing Sections, Dover, New York, NY.

[117] Swift, H., 2010, "A Review of the Literature Related to Potential Health Effects of Aircraft Noise," FAA/NASA/Transport Canada Center of Excellence, Partner-COE-2010-003.

[118] Walden, E. W., 2003, "Seal Assembly for Telescopic Hydraulic Cylinder," U.S. Patent US6,626,438B2. 


\section{APPENDIX A \\ MATLAB IMPLEMENTATION OF THE SEGMENTATION PROCESS}

ShapeChanger, a MATLAB-based application, implements the concepts presented in this dissertation. This application was used to perform all segmentations shown in this work. This appendix presents a step-by-step guide to its use.

\section{A-1 Generating Design Profiles}

Launching ShapeChanger at the MATLAB command prompt opens the windows shown in Fig. A-1. The first step is to generate design profiles. The design profiles may be uploaded, or they may be generated within ShapeChanger.

\section{Uploading Files}

"Upload File" is useful for design profiles generated via another application. Should this be desired, the $x, y$ data identifying the points on the design curves needs to be saved in a format that ShapeChanger can manipulate. This needs to occur prior to the initiation of ShapeChanger. Although this process works on any number of piecewise linear curves, each containing a different number of points, a simple example of performing this operation on two simple four- and five-point curves, labeled a and $\mathrm{b}$, is now shown. Note that the data for each curve is a $2 \times n$ matrix with the first row containing the $x$ data and the second row containing the $y$ data. 


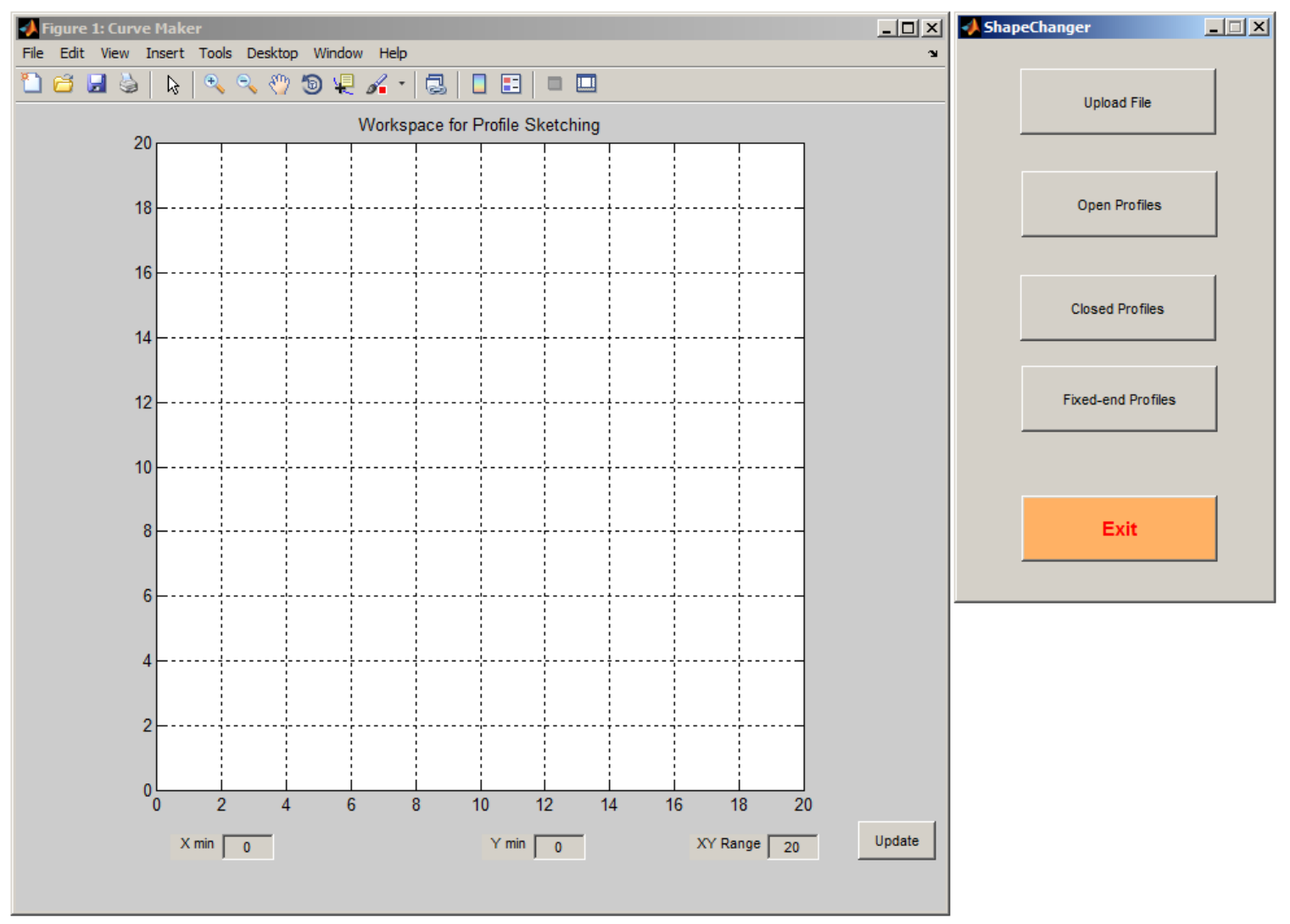

Figure A-1: The first two windows in ShapeChanger. 


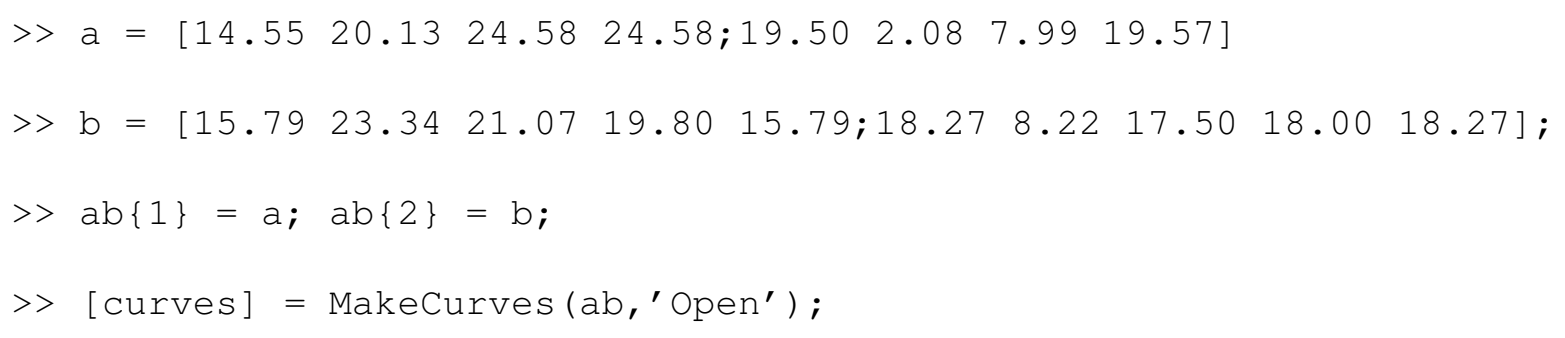

The last command precipitates two actions. First, it identifies the curves as being of the open-profile type. Second, a window to save the data opens, and the set of curves may be named and saved. Once complete, ShapeChanger may be initiated and the "Upload File" button used to exhibit these curves in the ShapeChanger environment. In addition to 'Open', the final field may also be 'Closed' or 'Fixed-end' identifying the curves as one of the other basic profile types.

Should the curves have been previously entered into MATLAB and saved in the appropriate format, "Upload File" may be used directly upon initiation. Note that the set of curves must contain two or more design profiles. After a successful upload, the "EDIT" button appears to move to the editing phase of generating design profiles.

The designer may also initiate the creation of design profiles via sketching the curves within ShapeChanger. In this case, one of the other options is selected from those shown in Fig. A-1: “Open Profiles," "Closed Profiles," or "Fixed-end Profiles." The steps for creating a set of design profiles of each type are enumerated below.

\section{Open Profiles}

1. Select "Open Profiles" as shown in Fig. A-1.

2. Select "Start Sketching".

3. Left click at the locations of the control points in the workspace. It is not necessary to be accurate as editing will follow.

4. Right click when done. 
5. To add another profile, click "Run Action" with the "Add Another Curve" button selected.

Draw in the workspace as described above.

6. Continue to add design profiles.

7. To proceed with editing, select the "Proceed to Edit" button and click "Run Action."

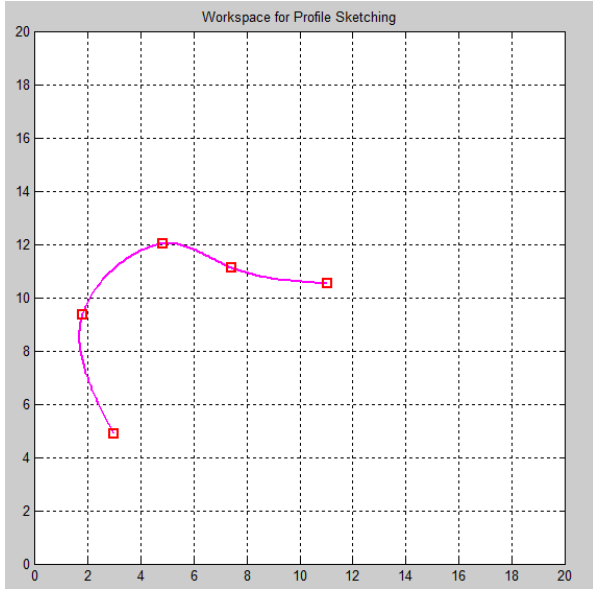

(a)

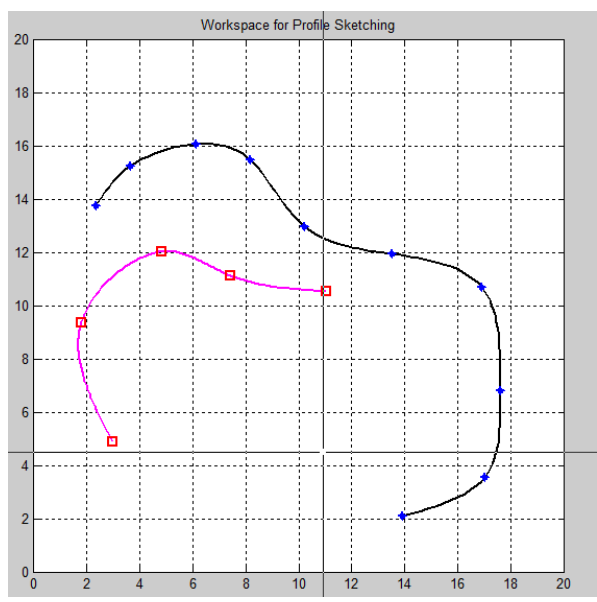

(c)

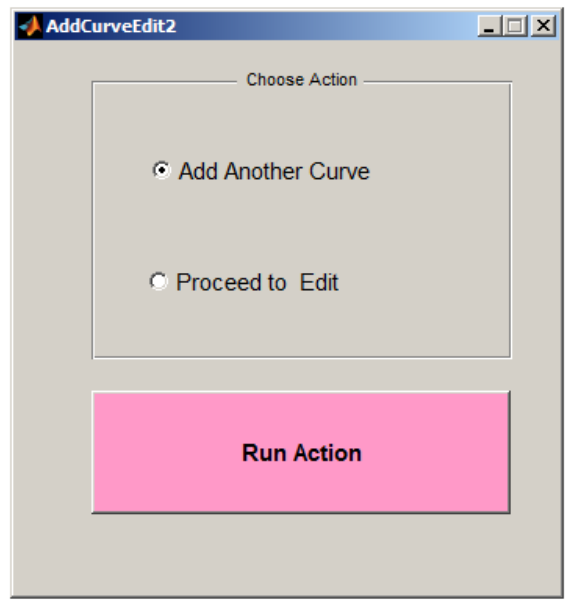

(b)

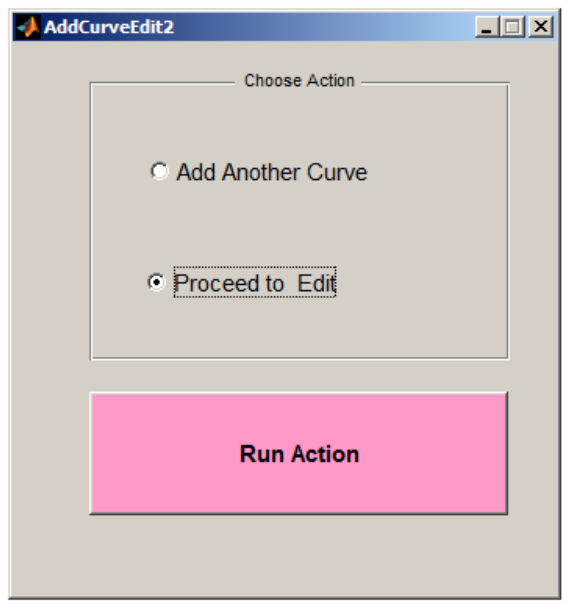

(d)

Figure A-2: (a) The first design profile is sketched. (b) Identifying that another profile is to be added. (c) The second design profile is sketched. (d) Proceed to the editing of the design profiles. 


\section{Closed and Fixed-End Profiles}

The steps for the other profile types are largely the same as those for open profiles. However, with the knowledge that curves will be closed or fixed-end, ShapeChanger adds some details. In the case of closed profiles, an example is shown in Fig. A-3. When the profile is near to completion, the final right click of the mouse automatically closes the profile

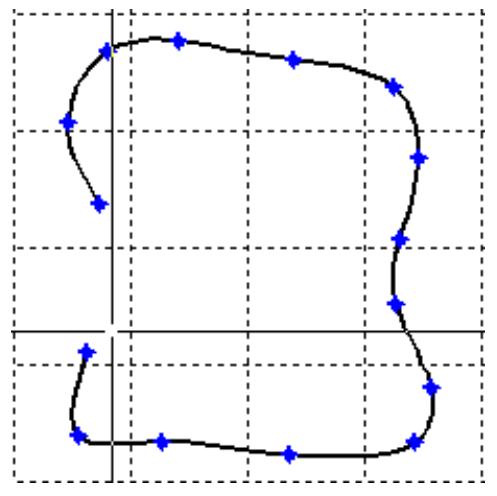

(a)

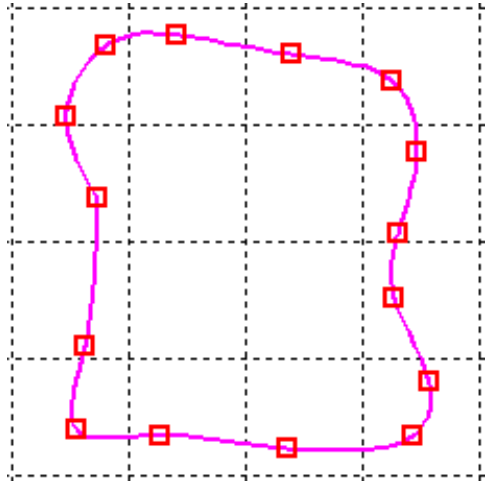

(b)

Figure A-3: (a) A closed design profile is generated in the workspace with left mouse clicks. (b) A right click closes and finishes the profile.

In the case of fixed-end profiles, the first and last points on the initial profile are used to identify the locations of the fixed ends. All additional profiles will assume the use of the same first point. The right click to end the profile will assume the use of the same last point. An example is shown in Fig. A-4.

\section{A-1.1 Editing Design Profiles}

The profiles may now be edited to refine features. The editing functions include the following: Move Points, Delete Points, Add Points, Translate, Rotate, and Scale All. Any of these functions are 


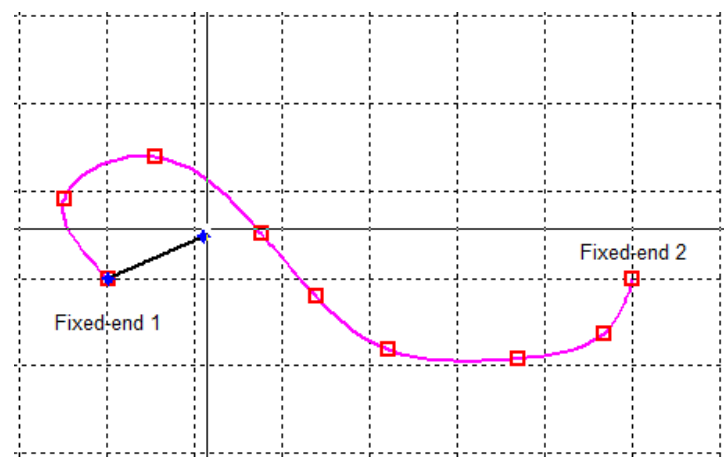

(a)

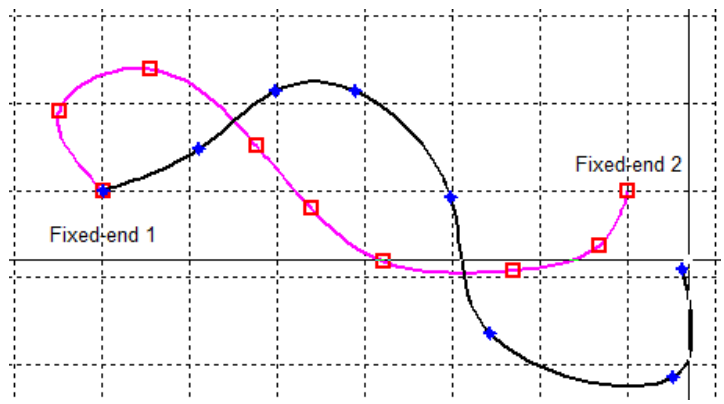

(b)

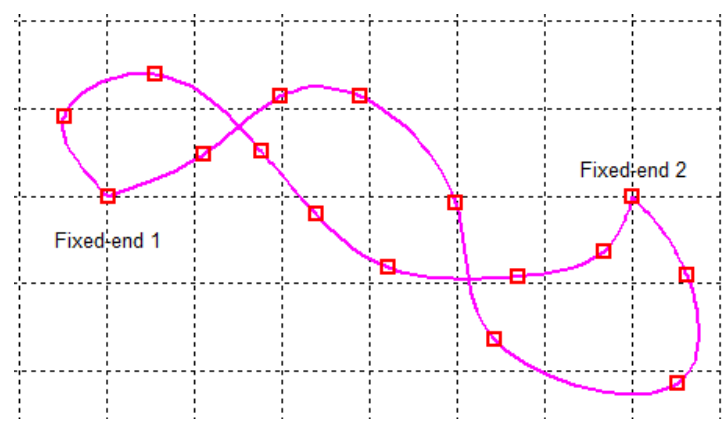

(c)

Figure A-4: (a) A fixed-end design profile is generated in the workspace with left mouse clicks. The second control point of the next profile is clicked. (b) The second profile adopts the same initial point as the first profile, and the connection to it is automatic. (c) The second profile adopts the same final point as the first profile, and the connection to it is automatic with the right click indicating completion of the profile. 
enacted by selecting the appropriate radio button and then clicking "PERFORM OPERATION". The relevant window is shown in Fig. A-5. Moving, adding and deleting points are functions performed on separate points in profiles to refine the features of part of the profile while leaving the rest of the profile (and all other profiles) intact. 'Translate' and 'rotate' move entire profiles to allow for the arrangement of relative profile location (a decision that does not affect segmentation but significantly influences mechanization). Finally, scaling operates on all curves and tends to be selected as the final editing operation when the desired design is to feature only $\mathscr{M}$-segments and revolute joints.

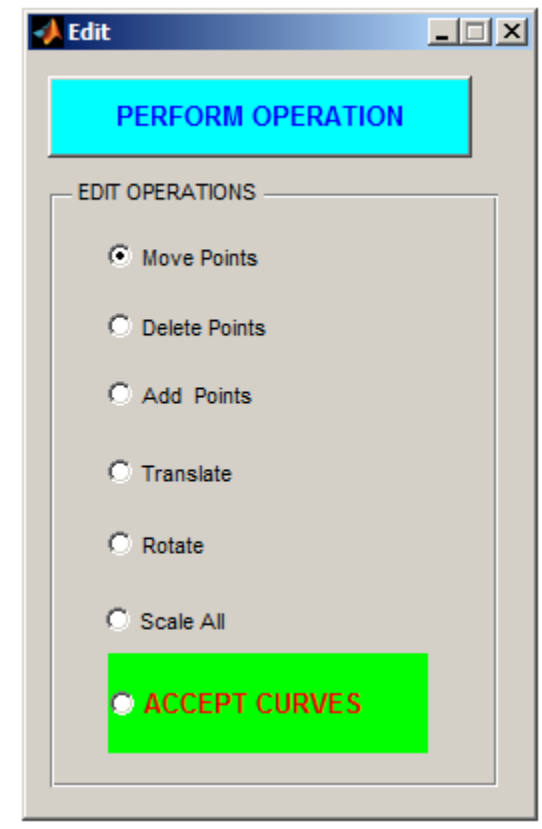

Figure A-5: Editing operations on the design profiles.

\section{Move Points}

1. Select the "Move Points" radio button.

2. Select "PERFORM OPERATION." 
3. Left click nearest a control point on the profile containing the point to be moved. This design profile becomes highlighted.

4. Left click nearest the control point to move. The point is indicated by a red box.

5. Left click the new location of the control point.

6. Left click near other points to highlight and then move, or right click to end the operation.

If another curve requires points to be moved, restart from the beginning.

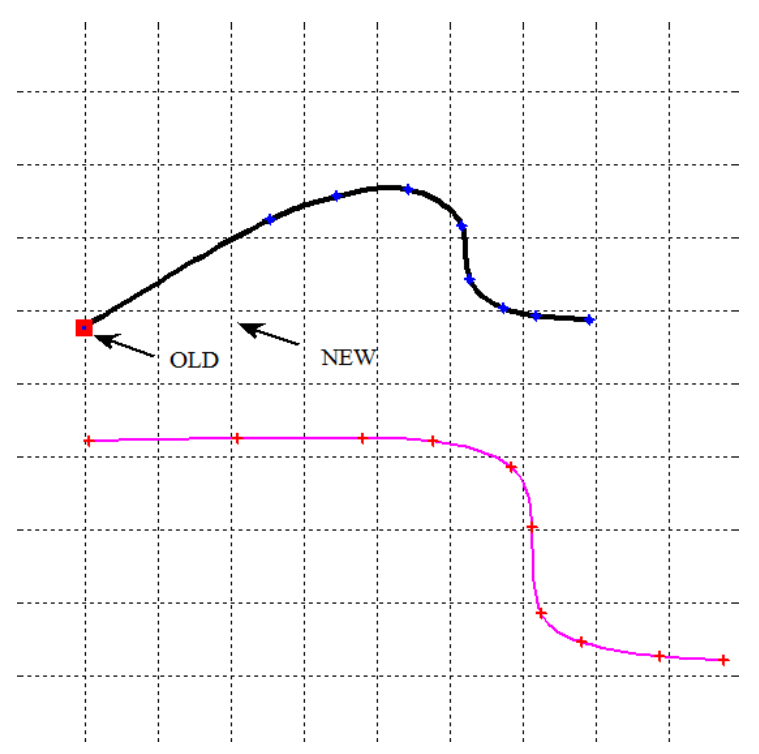

(a)

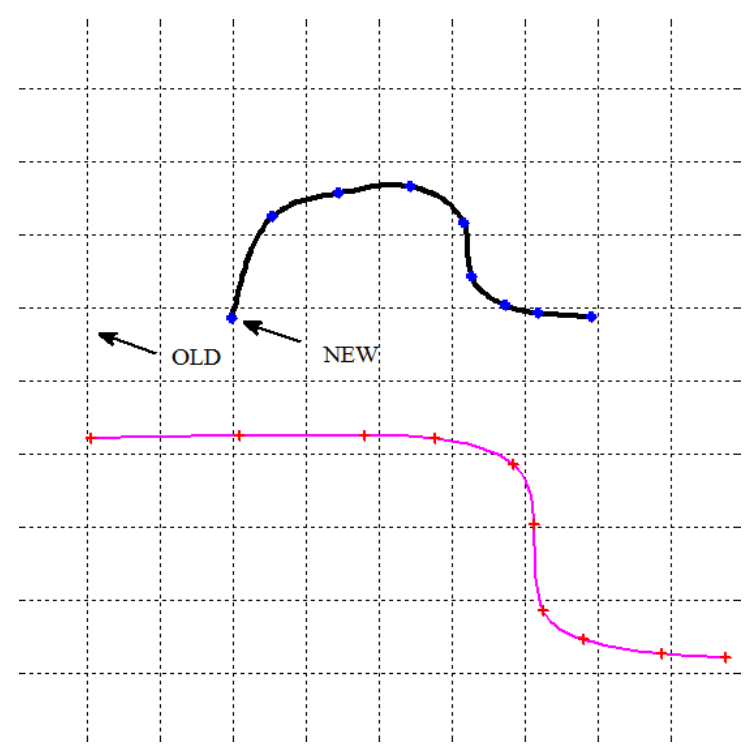

(b)

Figure A-6: (a) A click selects and highlights that profile. A second click indicates that point is to be moved. (b) The curve after a new location is selected for the point.

\section{Delete Points}

1. Select the "Delete Points" radio button.

2. Select "PERFORM OPERATION." 
3. Left click nearest a control point on the profile containing the point to be deleted. This curve becomes highlighted.

4. Left click nearest the control point to delete. The point disappears.

5. Left click other control point to delete, or right click to end the operation.

This tool is not meant to eliminate design profiles entirely, and doing so is a known bug. Should another curve require points to be deleted, restart the operation from the beginning.

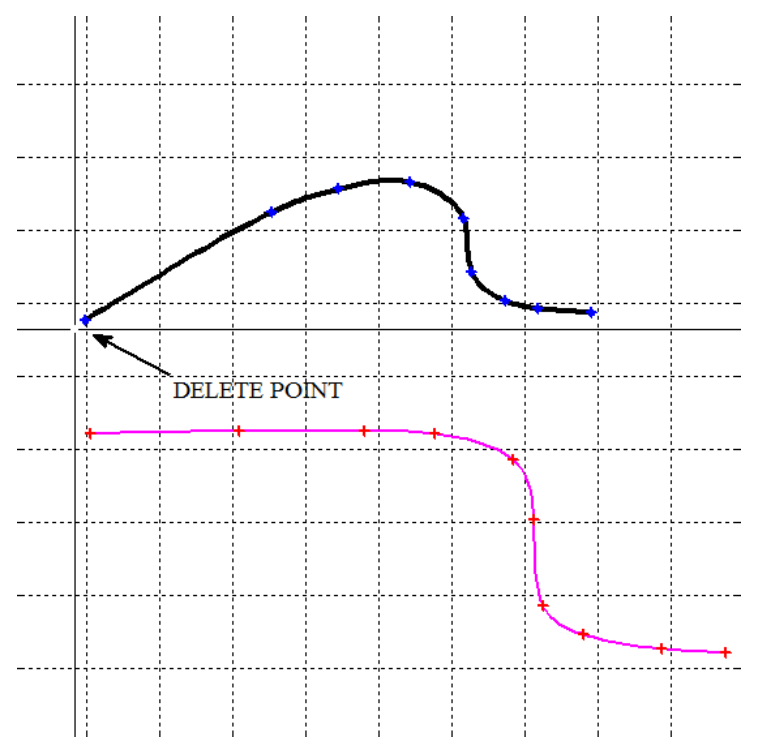

(a)

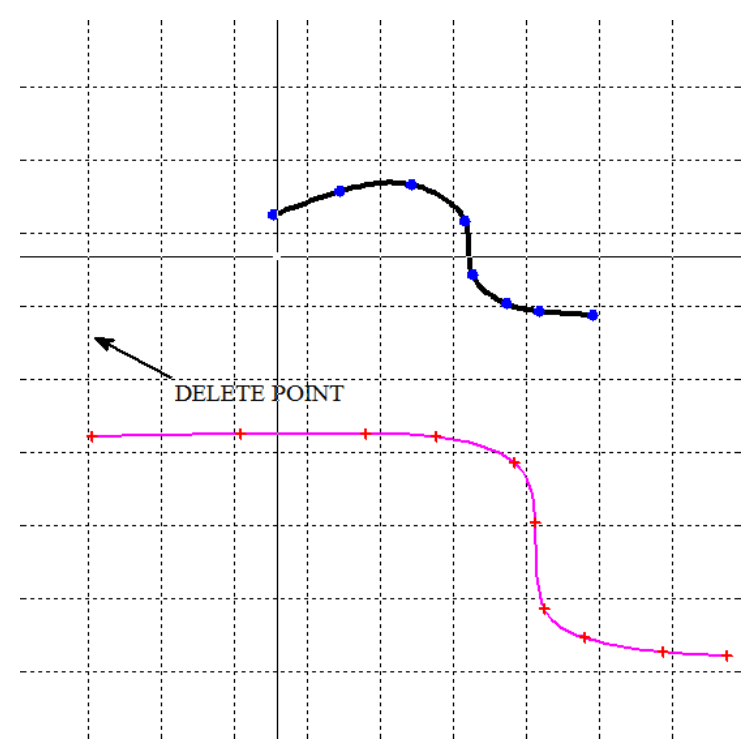

(b)

Figure A-7: (a) A click selects and highlights that profile. (b) A second click selects and deletes the control point.

\section{Add Points}

1. Select the "Add Points" radio button.

2. Select "PERFORM OPERATION." 
3. Left click nearest a control point on the profile containing the location of the point to be added. This curve becomes highlighted.

4. Left click on the profile at the location to add a point. The control points on the highlighted profile nearest that location are indicated in between two magenta squares.

5. Click again at the location to add, adding a control point between the magenta squares.

6. Continue to add points to that profile, or right click to end the operation.

If another curve requires points to be added, restart from the beginning.

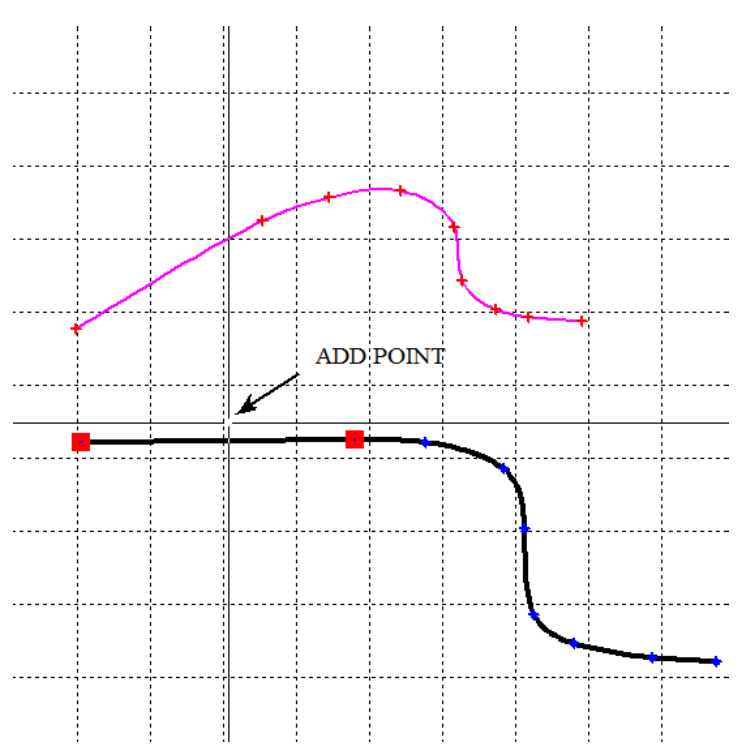

(a)

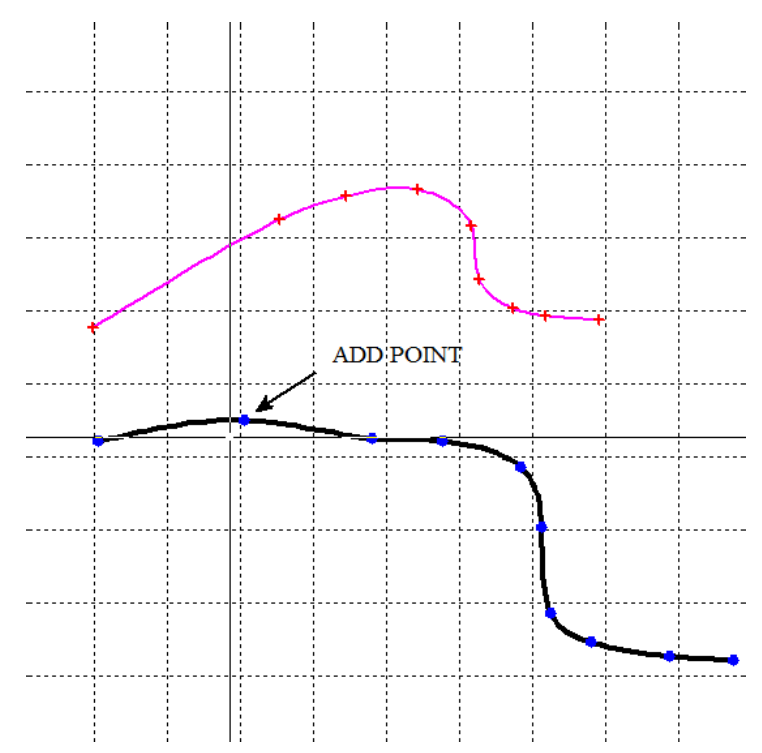

(b)

Figure A-8: (a) A click selects and highlights the profile. A second click indicates the range marked by two magenta squares. (b) If the range is right, select the exact location of the new point. Then the new point appears on the profile after the selection.

\section{Translate}

"Translate" may be selected to translate an entire profile to a new location. Note that the entire profile selected is moved as one entity. 
1. Select the "Translate" radio button.

\section{Select "PERFORM OPERATION."}

3. Left click nearest a control point on the profile containing the point to be moved. This curve becomes highlighted.

4. Left click nearest the control point as a reference. The reference point is circled in red.

5. Left click the new location of the reference point. The whole profile is translated accordingly.

6. Left click near another point to highlight and translate, or right click to end the operation.

If another curve needs to be translated, restart the operation from the beginning.

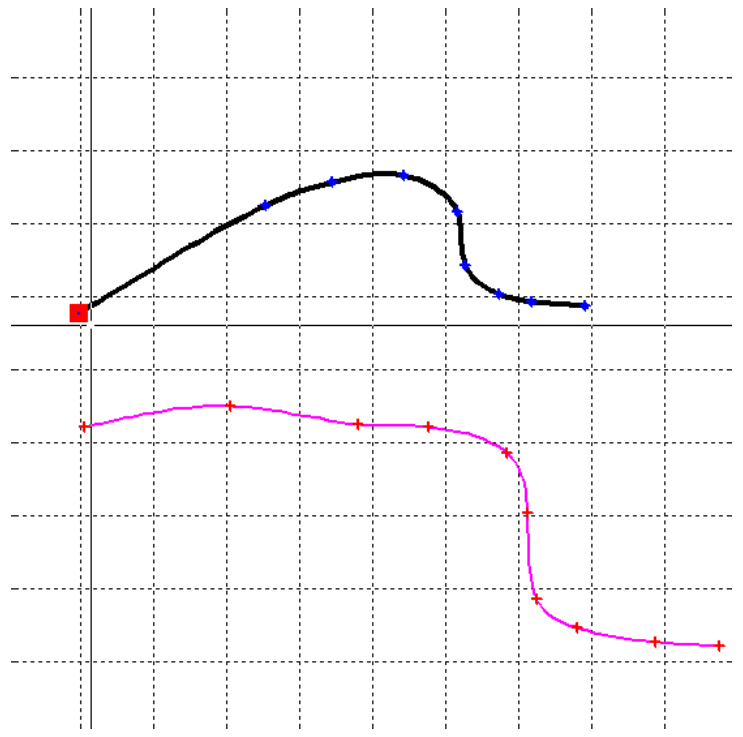

(a)

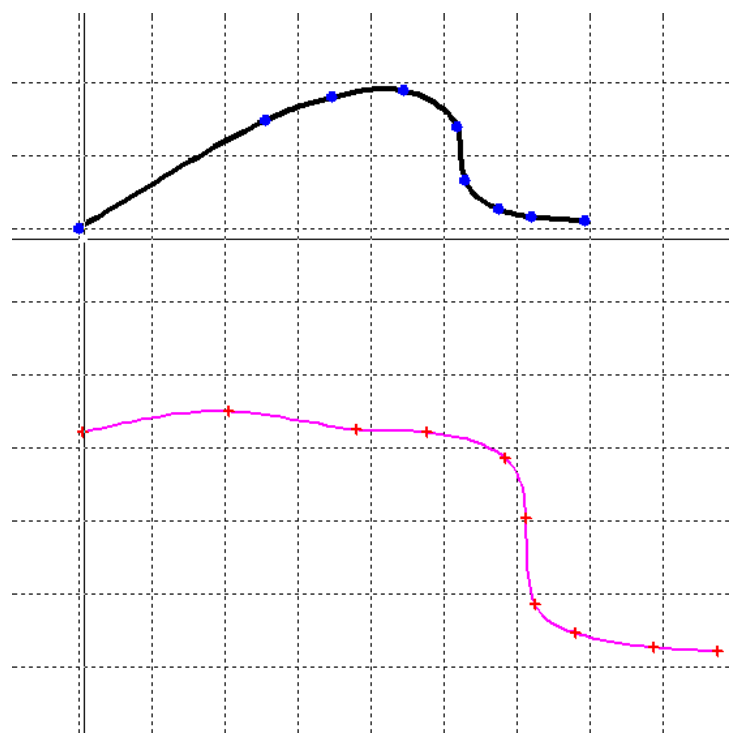

(b)

Figure A-9: (a) Select the curve and then the reference point (b) Click on the new location for the selected reference point on the profile to move the entire profile. 


\section{Rotate}

"Rotate" may be selected to rotate an entire profile about one of its control points. Note that the entire profile selected is rotated as one entity.

1. Select the "Rotate" radio button.

2. Select "PERFORM OPERATION."

3. Left click nearest a control point on the profile containing the point about which to rotate. This curve becomes highlighted.

4. Left click nearest the control point as a reference or rotational pivot. A red dot and a 'P' appear at that control point.

5. Enter the rotation angle in degrees and click OK. The whole profile is rotated accordingly.

6. Left click near another point as pivot. Then, enter the new angle, or right click to end the operation.

If another curve needs to be rotated, restart the operation from the beginning.

\section{Rescale All}

The "Rescale All" operation resizes all design profiles to have the same arc length as the design profile with the shortest arc length. This operation is useful when restricting design problems to be solved by a chain of $\mathscr{M}$-segments. Note that the user stays in the editing mode after this operation, and performing additional editing operations is likely to alter design profile arc lengths.

1. Select the "Rescale All" radio button.

2. Select "PERFORM OPERATION." 


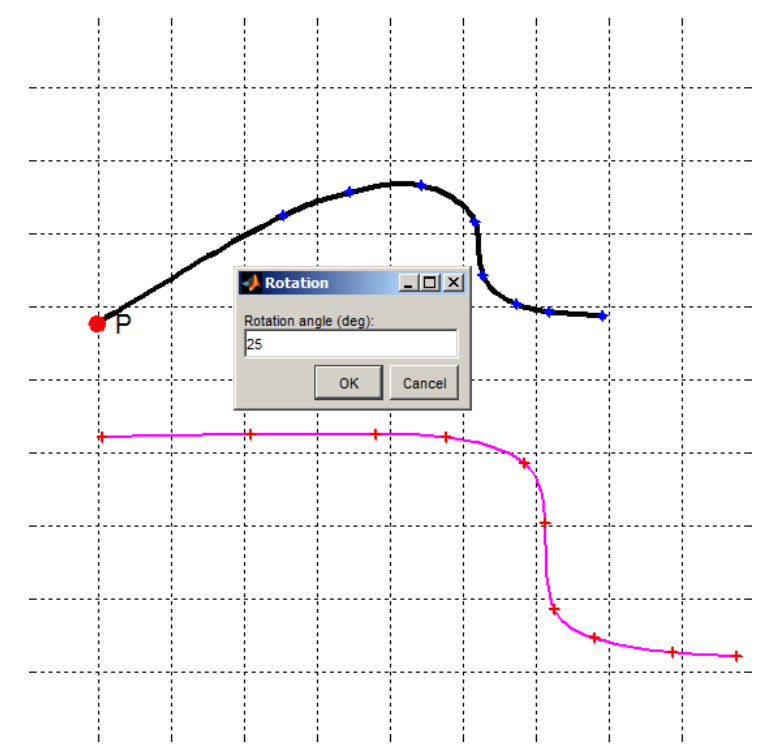

(a)

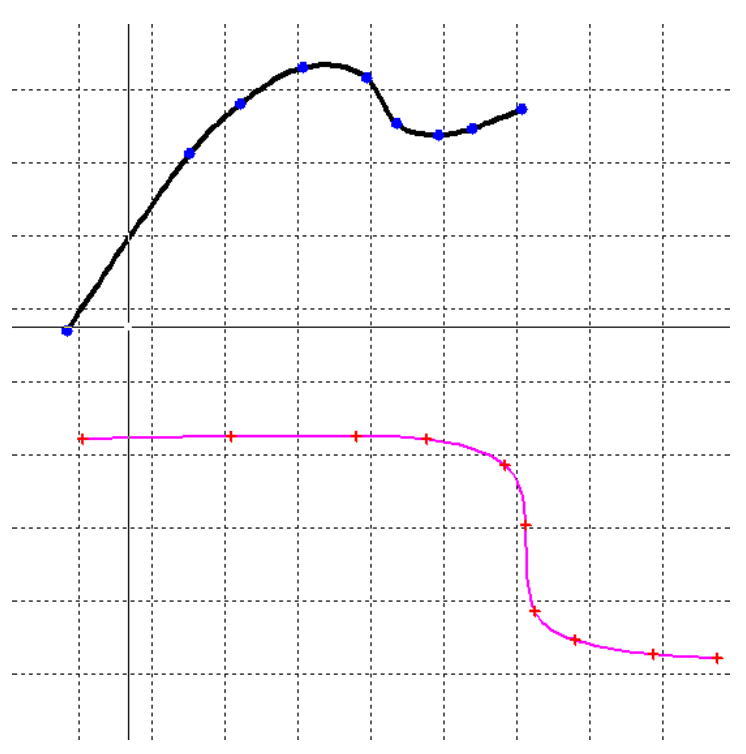

(b)

Figure A-10: (a) The top profile is selected, and a second click indicates the control point about which to rotate. A dialog box opens to input the angle of rotation. (b) The curve after a rotation about the selected control point.

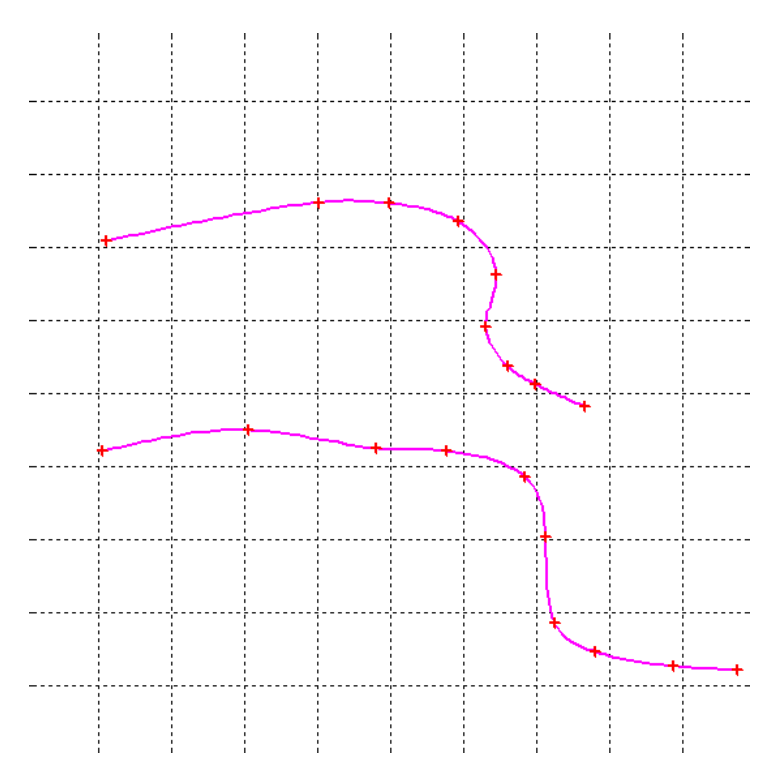

(a)

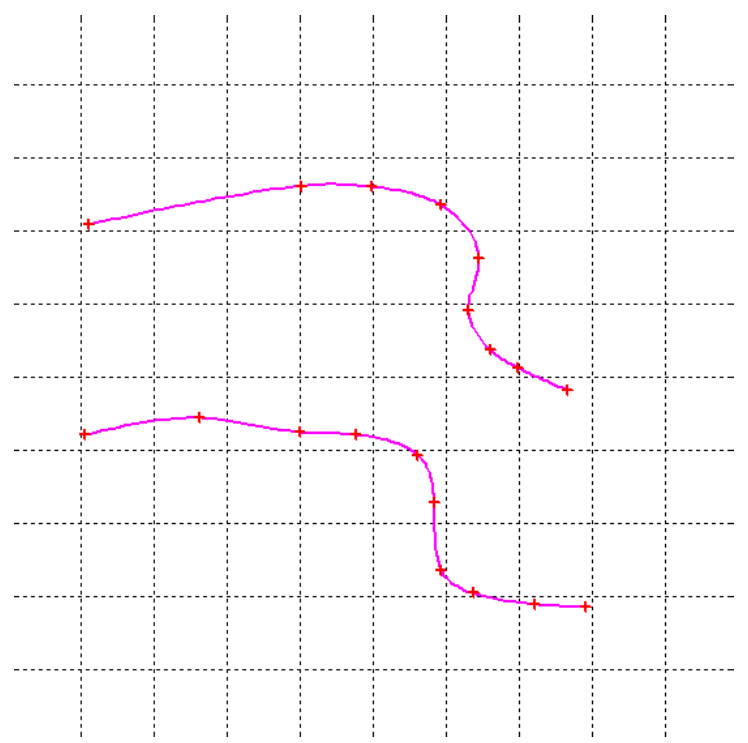

(b)

Figure A-11: (a) A pair of profiles is (b) rescaled to have the same arc length. 


\section{A-2 Generating Target Profiles}

When editing of the design profiles is complete, toggle the "ACCEPT CURVES" radio button shown in Fig. A-5 and then select "PERFORM OPERATION." Two windows will now open, the workspace and the control box. The workspace continues to exhibit the design profiles and the initial target profiles, while the control box contains information about the profiles. This pair of windows is shown in Figs. A-12 and A-13.

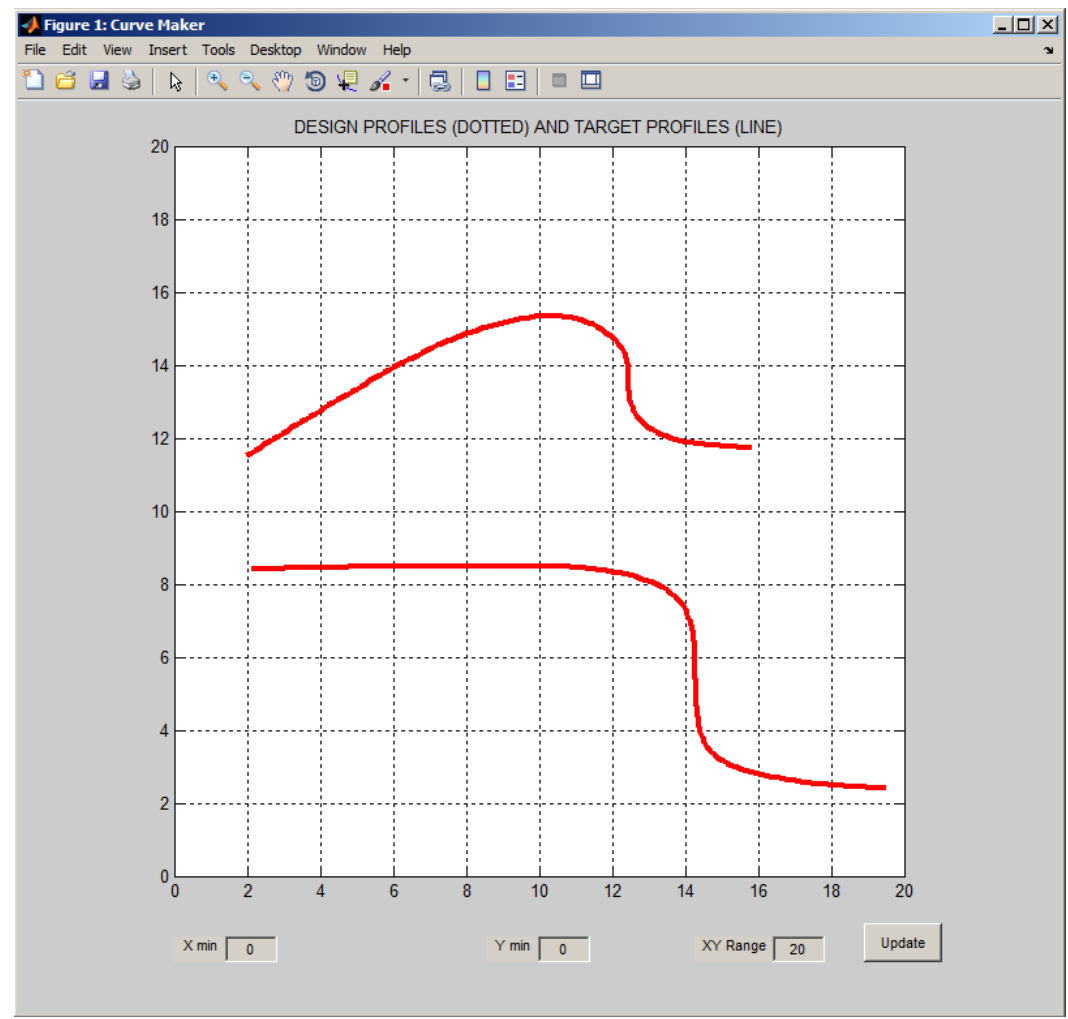

Figure A-12: The workspace presents the two target profiles matching well the design profiles beneath them. 


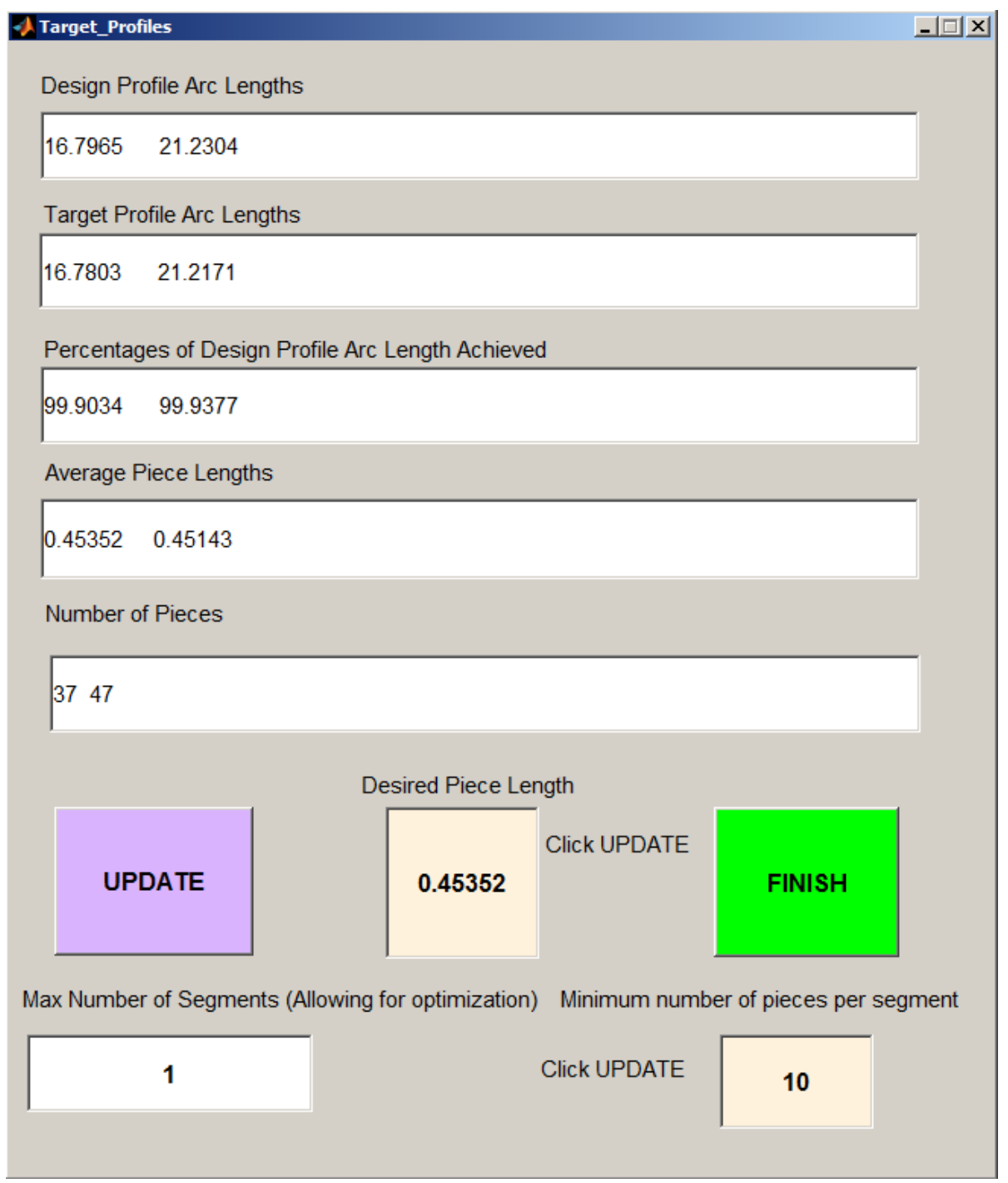

Figure A-13: The relevant information about target profiles influence the next phase, the segmentation. 
The workspace includes both the design profiles as blue dashed lines and the target profiles in red. Due to the accuracy of the match, the design profiles are difficult to see in Fig. A-12. Using less accurate target profiles, the design profiles are more readily visible in Fig. A-14.

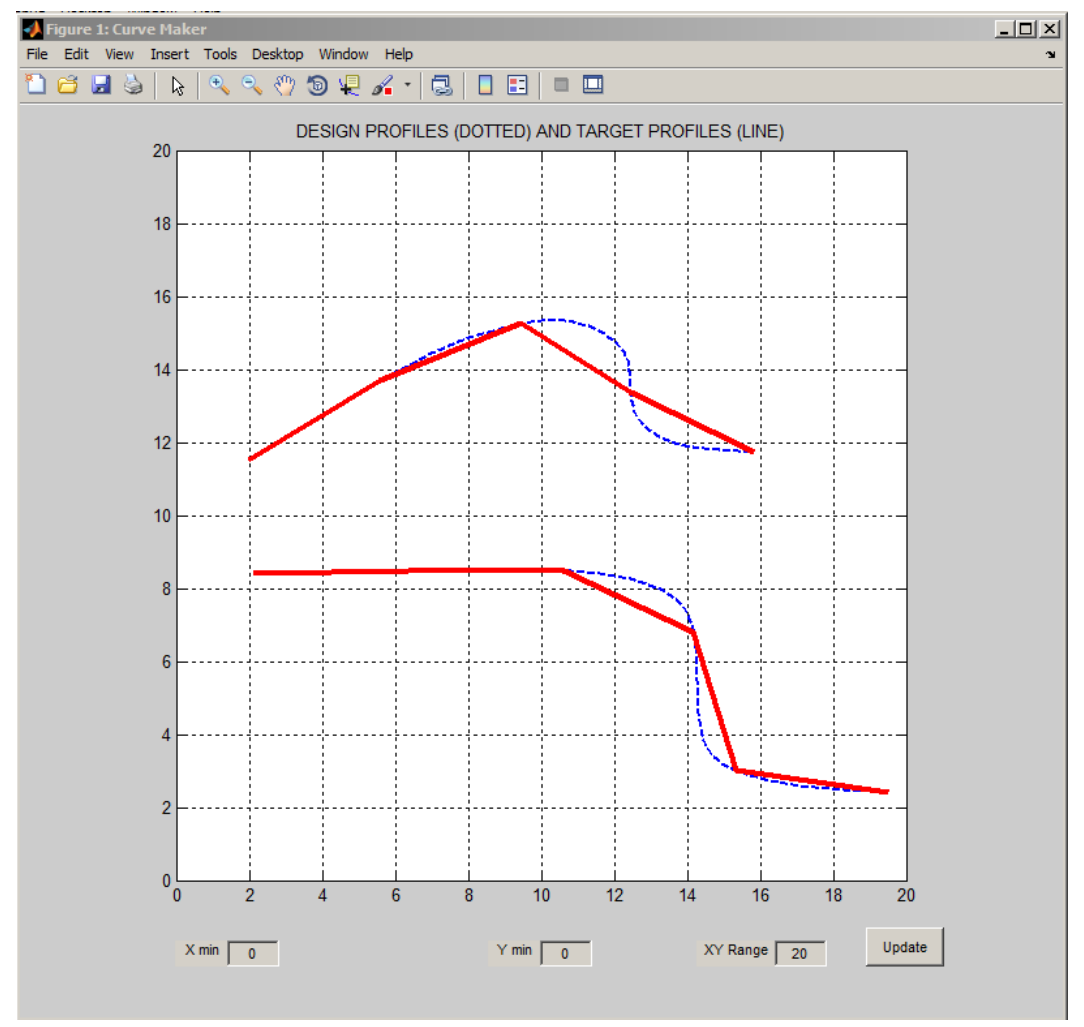

Figure A-14: A selection of desired piece length, $s_{d}=4$, that leads to a poor match between the design and target profiles.

The control box has information about the profiles as listed below.

1. Design Profile Arc Lengths: The total arc length of each design profile.

2. Target Profile Arc Lengths: The current total arc length of each target profile.

3. Percentages of Design Profile Arc Length Achieved: The percentage of each target profile's arc length over its design profile's arc length. 
4. Average Piece Lengths: The mean piece length of each target profile, which is approximately the same as the desired piece length.

5. Number of pieces: Number of line segments (pieces) that constitute each target profile. This number is one less than the number of points on each target profile.

6. Desired Piece Length: The ideal length of each piece on each target profile. The Average Piece Length is determined to match this as closely as can be achieved. Altering the Desired Piece Length changes the Target Profile Arc Lengths, Percentages of Design Profile Arc Length Achieved, and Number of Pieces.

7. Minimum Number of Pieces per Segment: The lowest allowable integer value for the number of pieces defining a segment. This is the value of $\alpha$ in Eq. 75 .

8. Max Number of Segments (Allowing for Optimization): The value of $q$ in Eq. 75, the maximum number of rigid-body segments that would allow for size optimization.

9. UPDATE: This button executes the changes made in either the "Desired Piece Length" or the "Minimum Number of Pieces per Segment" fields.

10. FINISH: This button ends the target profile generation phase, offers to save the data and initiates the segmentation process.

As indicated in the above list, the parameters in the target profile control box that can be changed are the Desired Piece Length, $s_{d}$, and the Minimum Number of Pieces per Segment, $\alpha$. Note that the control box in Fig. A-13 shows the initial target profile generation, automatically created with the conclusion of the design profile editing phase. In this case, the calculated value is $s_{d}=0.45352$. Briefly, at the beginning of this phase, this value is calculated from redistributing points on the design profiles to achieve as close to $99.9 \%$ of design profile arc lengths per profile as can be generated. This results in $\bar{s}_{1}=0.45352$ and $\bar{s}_{2}=0.55818$ (not displayed). The minimum of these is the suggested $s_{d}$. However, the points are then automatically redistributed to achieve this 
$s_{d}$ and result in $\bar{s}_{1}=0.45352$ and $\bar{s}_{2}=0.45143$. Changing the Desired Piece Length to 4 and pressing "UPDATE" generates the result in Fig. A-14. This process only redistributes points with the objective to approximate $s_{d}$. Close attention should be paid to the 'Percentages of Design Profile Arc Length Achieved' field to verify that an acceptable match of the design profiles is being generated.

The default minimum number of pieces in any segment is 10 . There is an interaction between the number of pieces and the number of segments. As the $s_{d}$ is changed, the physical meaning of $\alpha$ changes. Hence, instead of specifying an $\alpha$ value, a practical minimum segment size should be identified and the value of $\alpha$ adjusted accordingly.

\section{A-3 Segmentation}

The segmentation process starts by taking the input parameters given from the target profile generation phase. Figure A-15 shows three parameters, the number of segments per profile, the minimum number of pieces per segment, and number of pieces per profile. The value ' 10 ' is the default for the minimum number of pieces per segment unless otherwise specified in the target profile generation phase. The values in the 'Number of Pieces per Profile' field are fixed but can be changed by regenerating the target profiles.

Changes are still possible provided they are consistent. Consistency is achieved by considering the number of pieces that are on each target profile before changing the number of segments. Should more segments be needed than what is suggested, the minimum number of pieces per segment needs to be reduced. For instance, if profile 1 has 100 pieces and 5 segments are specified, then according to Eq. 75 , the $\alpha$ can be a maximum of 10 . However, if 4 segments per profile is chosen, the maximum value of $\alpha$ is 12 . 


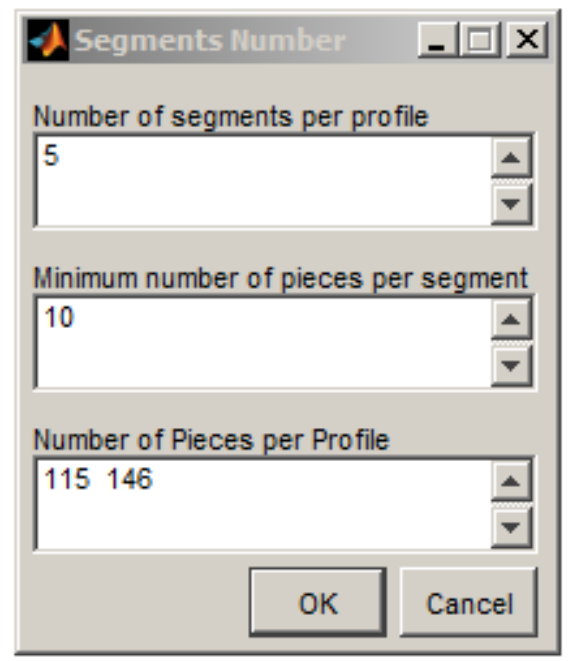

Figure A-15: Segmentation process takes the input from this window.

Special attention is given to closed profiles. The start point of each target profiles can be changed such that the resulting rigid-body segments are likely to approximate the target profiles well. Figure A-16a shows the option to change the start point for the two closed profiles in Fig. A-16b. Start and end points of the closed profiles are fixed locations and do not change during optimization. Hence, these end points should be locations where the resulting segments would not 'flip' and produce serious mechanization challenges. Figure A-16c shows new start points for the two airfoils.

The next window, shown in Fig. A-17a, offers different plot options at the three stages of the segmentation process. Selecting "See Optimization", the changes in segments' lengths during optimization are plotted for all design vectors. For instance, if the design vector is $\mathbf{V}=$ $[\mathscr{C} \mathscr{M} \mathscr{M} \mathscr{M} \mathscr{M}]$, for this design alone, several initial segment matrices are generated. Each segment matrix will undergo the error reducing optimization algorithm in order to search for the lowest $E_{\max }$. The program stops generating initial segment matrices when the error minimizing $E_{\max }$ could not be improved in five iterations after the lowest $E_{\max }$ is recorded. This process is repeated with other V's or chain designs in search of the most suitable result. 


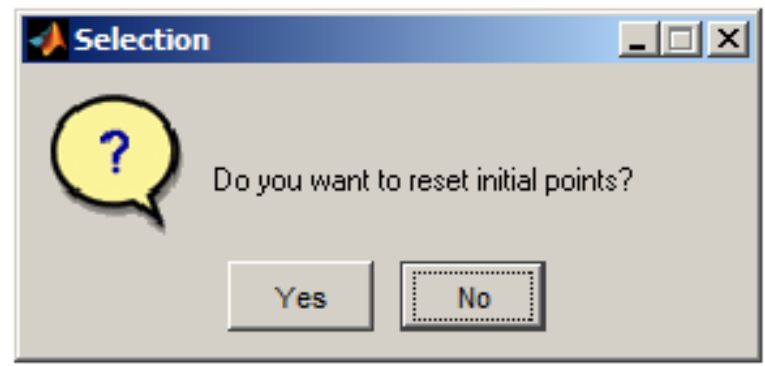

(a)

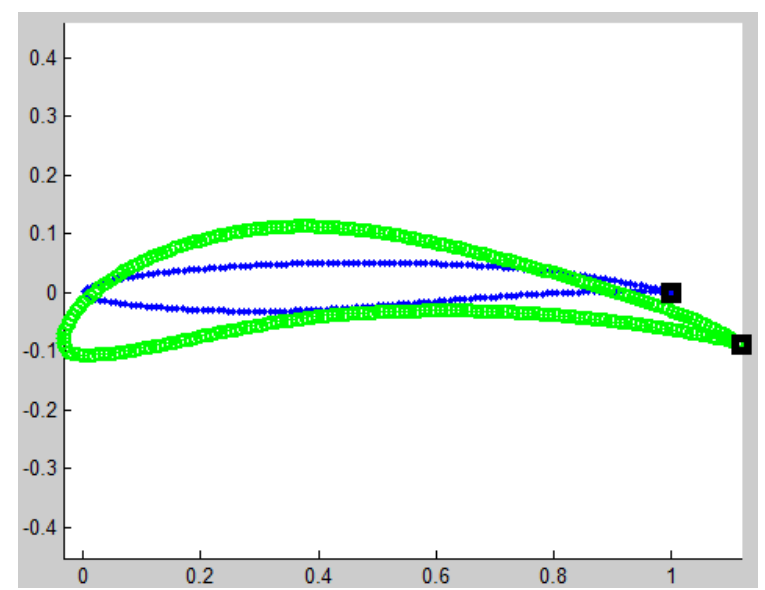

(b)

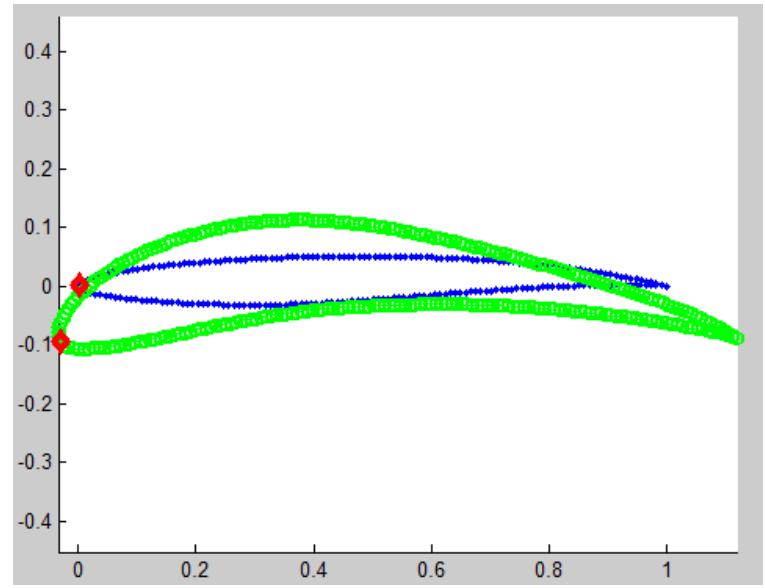

(c)

Figure A-16: (a) Reset starting points dialog box. (b) Original starting points of the design profiles. (c) New starting points for the target profiles. 
Selecting "Skip Seeing Optimization" leads to another two options. Choosing "View All Design Vectors," as in Fig. A-17b, will only display one plot corresponding to the $\mathbf{V}_{1}$ design with the minimum error of $E_{V_{1}}$. However, for a five-segment chain with at least one $\mathscr{C}$-segment, there are $B=2^{5}-1=31$ design vectors to explore. Hence, this option will display the results of these 31 designs before showing the overall error minimizing design.

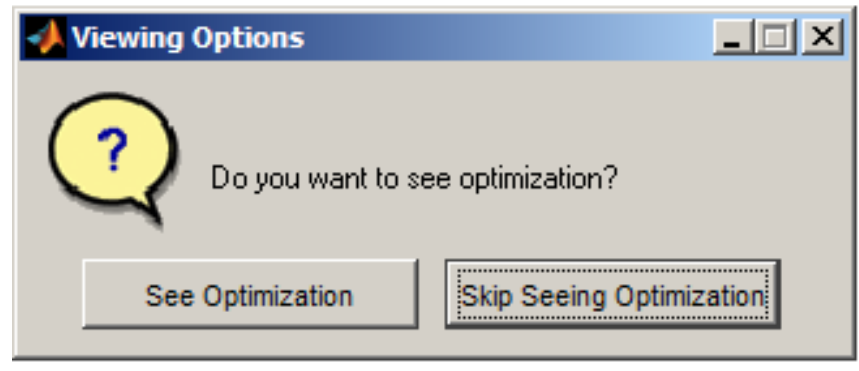

(a)

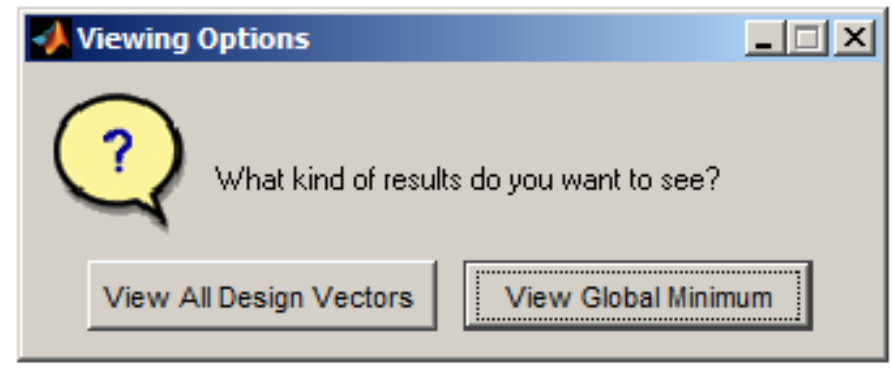

(b)

Figure A-17: (a) A user can set whether to see the optimization process changing the segments' lengths, or (b) to see the result of each chain design, or just see the final result.

In Fig. A-17b, seeing only the overall error minimizing design can be selected by choosing "Global Minimum." Here, the lowest $E_{\max }$ from all the designs can be compared, such as $E_{\text {final }}=$ $\min \left(E_{V_{1}}, \ldots, E_{V_{B}}\right)$. Hence, the segmentation process proceeds as described in detail in Chapter III. 
Finally, the design with the lowest error is selected, as shown in Fig. A-18. However, designers might have other criteria in determining the overall error minimizing design. Hence, there is the need for other results from various designs to be studied as well.

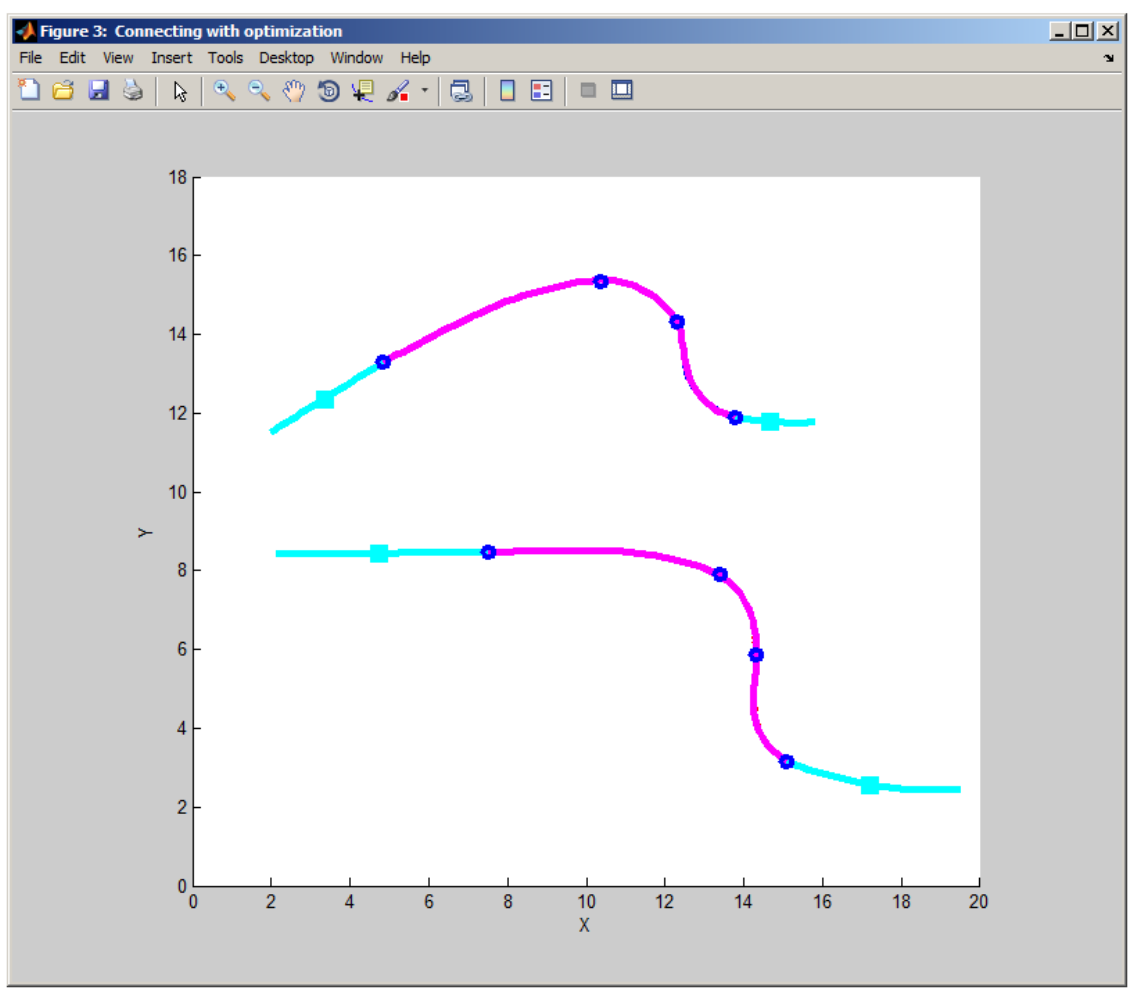

Figure A-18: A result from the segment synthesis that is selected due to it having the lowest maximum point-to-point error, $E_{\max }=0.09$.

Compound segments can be integrated if the variation of a joint angle between two segments is deemed insignificant. Explanation on this issue can be found in Sections 3.4 and 4.5. The dialog box in Fig. A-19a displays the range of rotation at each revolute joint, and the fusing angle set at $11^{\circ}$. The fusing is shown in Fig. A-19b. This will increase the error. It is up to a designer to decide whether using compound segments is beneficial. 


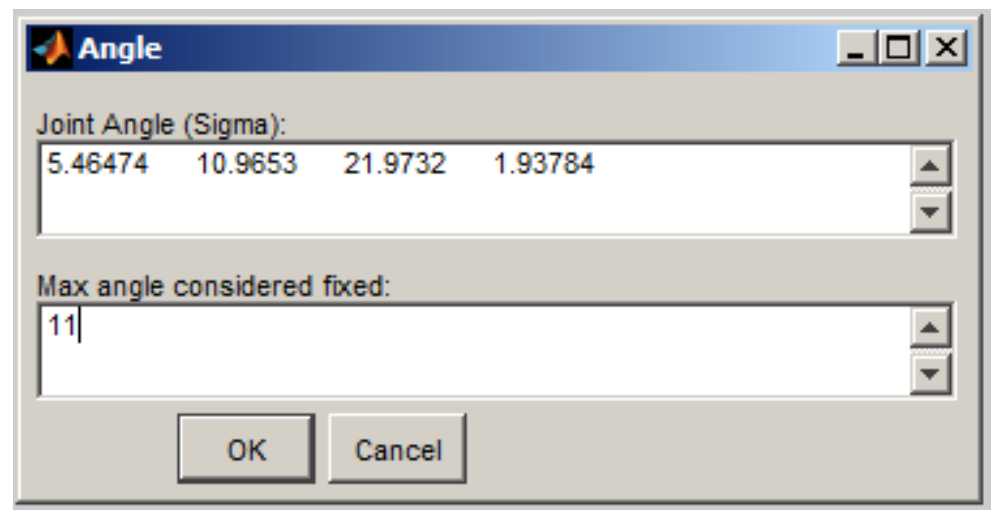

(a) Joint angles box

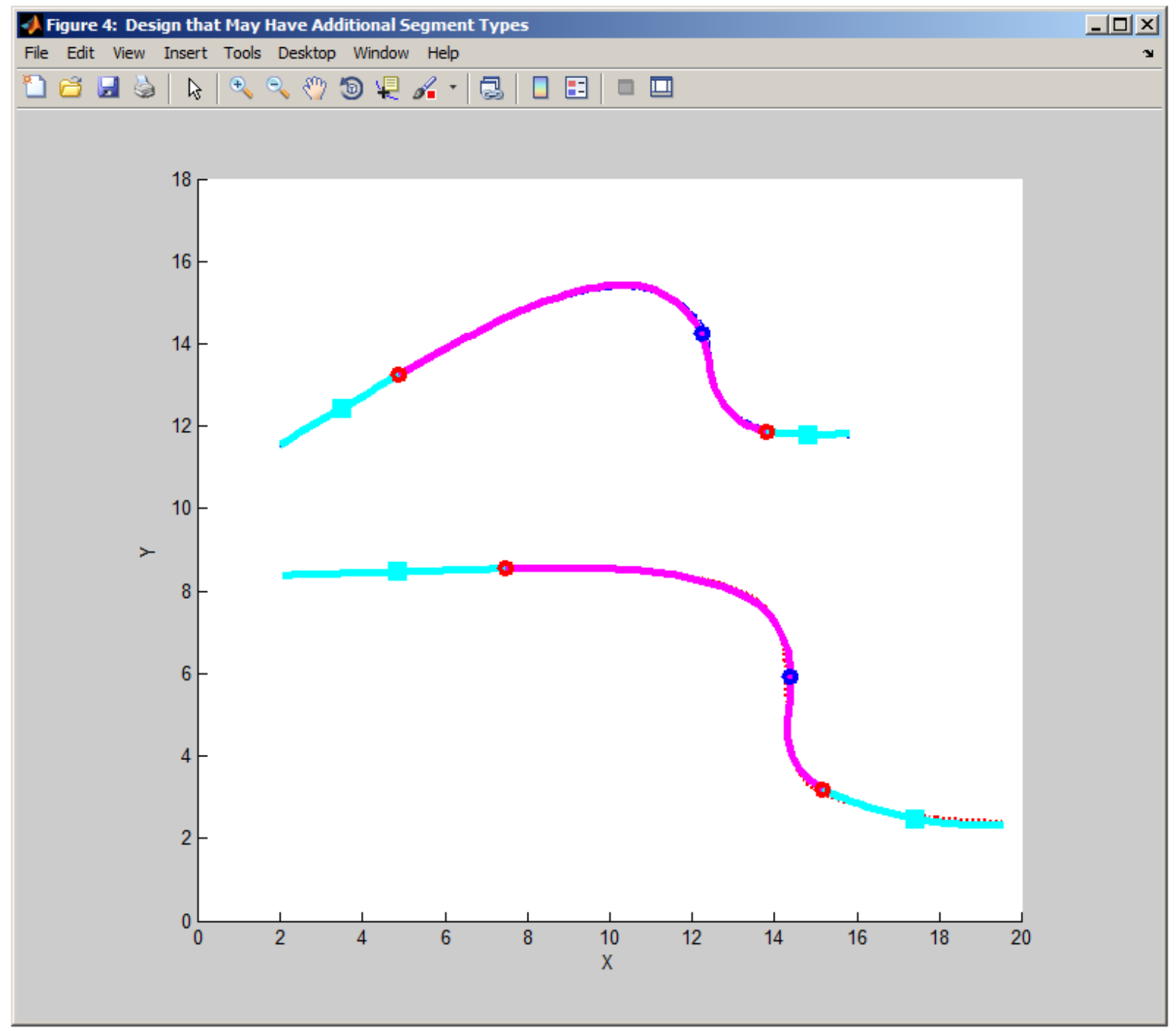

(b) $E_{\max }=0.13$

Figure A-19: (a) Maximum angle variations at revolute joints are shown and an allowable angle is set. (b) Fusing segments can reduce complexities. 